\title{
The influence of walking aids on the recovery of gait function and balance following stroke
}

Citation for published version (APA):

Maguire, C. (2016). The influence of walking aids on the recovery of gait function and balance following stroke. [Doctoral Thesis, Maastricht University]. Datawyse / Universitaire Pers Maastricht. https://doi.org/10.26481/dis.20161019cm

Document status and date:

Published: 01/01/2016

DOI:

$10.26481 /$ dis. $20161019 \mathrm{~cm}$

Document Version:

Publisher's PDF, also known as Version of record

\section{Please check the document version of this publication:}

- A submitted manuscript is the version of the article upon submission and before peer-review. There can be important differences between the submitted version and the official published version of record.

People interested in the research are advised to contact the author for the final version of the publication, or visit the DOI to the publisher's website.

- The final author version and the galley proof are versions of the publication after peer review.

- The final published version features the final layout of the paper including the volume, issue and page numbers.

Link to publication

\footnotetext{
General rights rights.

- You may freely distribute the URL identifying the publication in the public portal. please follow below link for the End User Agreement:

www.umlib.nl/taverne-license

Take down policy

If you believe that this document breaches copyright please contact us at:

repository@maastrichtuniversity.nl

providing details and we will investigate your claim.
}

Copyright and moral rights for the publications made accessible in the public portal are retained by the authors and/or other copyright owners and it is a condition of accessing publications that users recognise and abide by the legal requirements associated with these

- Users may download and print one copy of any publication from the public portal for the purpose of private study or research.

- You may not further distribute the material or use it for any profit-making activity or commercial gain

If the publication is distributed under the terms of Article $25 \mathrm{fa}$ of the Dutch Copyright Act, indicated by the "Taverne" license above, 
The influence of walking aids on the recovery of gait function and balance following stroke 


\title{
The influence of walking aids on the recovery of gait function and balance following stroke
}

\author{
Dissertation \\ To obtain the degree of Doctor at Maastricht University, \\ on the authority of the Rector Prof. dr. Rianne M. Letschert \\ in accordance with the decision of the Board of Deans, \\ to be defended in public \\ on Wednesday 19th October 2016, at 10.00 hours
}

by

Clare C. Maguire 
Supervisor:

Prof.dr R.A.de Bie,

\section{Co-supervisor}

Dr. Judith M. Sieben

\section{Assessment Committee}

Prof.dr.C.G. Farber - Chair

Prof.dr.H. Saveleberg

Prof.de.Ria Nijhuis-van der Sanden

Dr. M. Wirz 


\section{Contents}

Chapter 1 General Introduction

Chapter 2 The influence of walking aids on the plasticity of Spinal Interneuronal Networks, Central Pattern Generators and the recovery of gait post-stroke. A review of the literature.

Chapter 3 Movement goals stored in the cortex and muscle synergies to reduce redundancy - an efficient system to control movement and gait. How does it work, what changes following stroke and what are the consequences for rehabilitation and the use of walking-aids? A literature review.

Chapter 4 Hip abductor control in walking following stroke - the immediate effect of canes, taping and TheraTogs on gait.

Chapter 5 Walking with a four wheeled walker (rollator) significantly reduces EMG lower-limb muscle activity in healthy subjects.

Chapter 6 The effect of crutches, an orthosis TheraTogs and no walking aids on the recovery of gait in a patient with delayed healing post hip fracture: a case report.

Chapter 7 How to improve walking, balance and social participation following stroke: a comparison of the long term effects of two walking aids canes and an orthosis TheraTogs - on the recovery of gait following acute stroke. A study protocol for a multi-centre, single blind, randomised control trial.

Chapter 8 An orthotic garment improves gait and balance when replacing canes in chronic, previously cane using stroke-patients. A replicated, single case experimental study.

Chapter 9 General Discussion

Summary

Valorization

Acknowledgements

About the Author Curriculum Vitae

Publications 

Chapter

1

General Introduction 



\section{Stroke prevalence and the burden of disease}

Stroke is the second leading global cause of acquired disability in adults. A Global Burden of Stroke Study published in 2014 estimated that stroke survivors worldwide number 33 million. [1] Disability Adjusted Life Years (DALYs) lost, in this case meaning the years of productive life lost due to disability caused by stroke, was estimated to be 102 million in 2010. Between 1990 and 2013 the global burden of stroke increased continuously, influenced by both population growth and ageing.[2] Importantly, most of the burden of stroke has been shown to fall on resource-poor, low and middle income countries $(68.6 \%$ of incident strokes, $52.2 \%$ of prevalent strokes, $70.9 \%$ of stroke deaths, and $77.7 \%$ of DALYs lost). [1] It is now estimated that $80 \%$ of all stroke survivors live in low and middle income economies. [3] Although the incidence of stroke is decreasing in high income countries, dropping 12\% between 1990 and 2010, it is increasing in low to middle income countries, showing a $12 \%$ rise in the same period. [1]

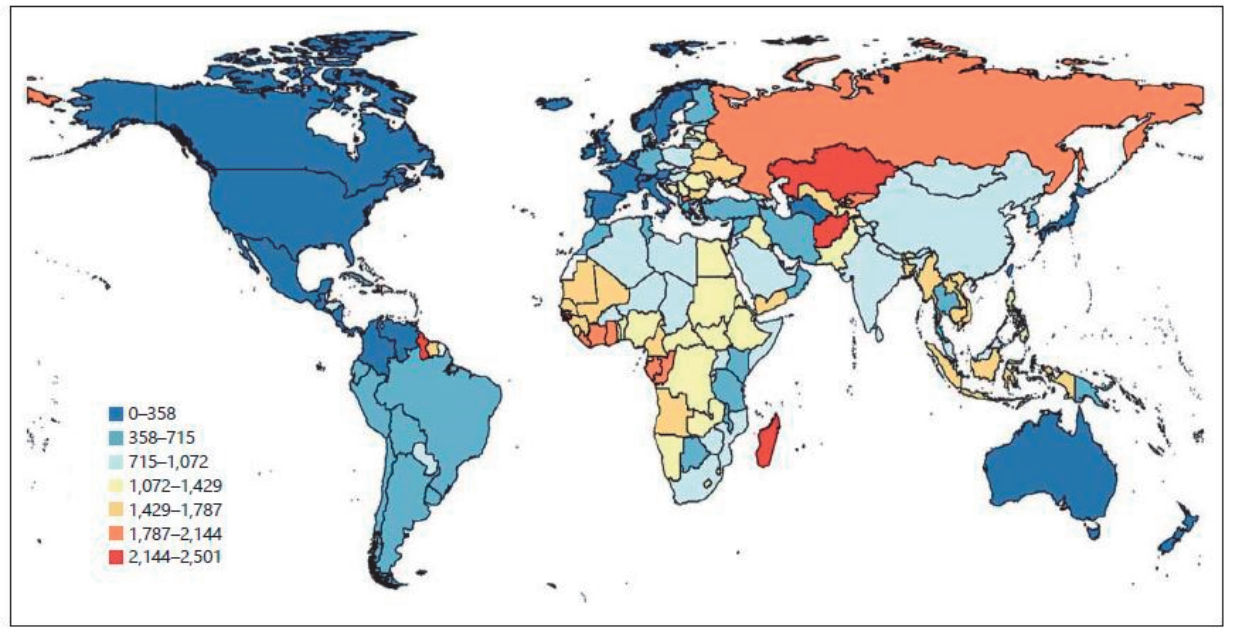

Figure 1. Age-standardized annual DALYs rates (per 100,000) due to ischemic stroke in 2013. Reproduced from Feigin VL (2015) Atlas of the Global Burden of Stroke [2]

These Global Burden of Stroke figures emphasise the need for more effective rehabilitation interventions to reduce disability and increase independence. As the majority of stroke survivors worldwide live in countries where, according to the World Stroke Organisation "health systems are already challenged to the limit"[4], the development of inexpensive, evidence based treatments should be a priority.

\section{Resulting disability and post-stroke complications}

Of those patients who survive stroke, approximately $65 \%$ are left with some form of disability. [5] At 6 months post-stroke it has been observed that 50\% have a hemipare- 
sis, $30 \%$ are unable to walk without some assistance, $26 \%$ are dependent in activities of daily living, $26 \%$ are institutionalized in a nursing home and $35 \%$ have depressive symptoms. [6]

Primary neurological changes post-stroke such as hemiplegia, reduced muscle tone, reduced sensation or perceptual changes contribute directly to some of these disabilities. Others are a result of secondary complications which may begin hours after stroke or develop in the following weeks and months. Immobility and loss of balance due to primary neurological changes may contribute to the development of deep vein thromboses (DVT) and post-stroke infections. [7] Structural changes within muscles are known to begin approximately 4 hours after stroke, with muscle weakness developing in both the paretic and non-paretic limb within the first week. [8] Immobility is likely to increase these changes and leads to further secondary complications such as loss of cardiovascular fitness. These changes increase the likelihood of falls and fractures, which in turn may lead to sarcopenia. [5] It has been shown that levels of ambulation and cardiovascular fitness are extremely low in chronic stroke survivors. Various mobility deficits, particularly poor balance, appear to contribute to low activity levels. [9]

This typical cascade of reactions in which poor mobility and balance lead to increasing muscle weakness, low levels of fitness, high levels of disability, dependence and depression, illustrate that post-stroke rehabilitation should not only attempt to treat the primary symptoms of stroke, but also to prevent the development of secondary complications largely responsible for long-term disability. Interventions which improve balance, increase muscle strength and cardiovascular fitness may be of particular importance for reducing or preventing disability and dependence.

\section{The development of effective treatments}

Although many forms of treatment exist for stroke rehabilitation, few are based on robust evidence. Interventions which are consistently highly recommended in clinical guidelines or systematic reviews are Constrained Induced Movement Therapy (CIMT) and robot assisted training for the upper limb, [10-13], task oriented and repetitive task training for upper \& lower limb and gait, [10-13] electromechanical- assisted gait training, [10-13] and strength and endurance training for upper and lower limb function and gait. [10-13] The development of strength and endurance training was based on knowledge of physiological muscular and cardiovascular processes in healthy and post stroke individuals. $[14,15]$ All other interventions were based on knowledge of fundamental neural and neuromuscular processes in healthy subjects and following stroke. This understanding was often gained from animal studies, from which theories of potential clinical applications were developed and later tested in clinical trials. For example CIMT was developed from an understanding of structural and functional cortical plasticity and factors affecting motor learning post-stroke, gained from studies with monkeys and rats. [16-20] Rehabilitation interventions were developed and tested initially in 
animals, followed by clinical trials. [21-23] Task oriented and repetitive task training interventions followed a similar development, in which insight into motor skill acquisition, gained in animal and psychological studies [16, 24-26], was applied in clinical practice and in clinical studies leading to effective, evidence based treatments. [27-30] Equally, electromechanical-assisted gait training was originally developed for use with spinal cord injured patients (SCl), based on a physiological understanding of neural mechanisms of gait control.[31, 32] These principles were later applied to develop robotic-assisted gait training for stroke patients, which was then tested in clinical trials. $[33,34]$ These results suggest that rehabilitation interventions are most effective when based on a sound understanding of the physiological and neural processes involved in movement control and motor learning in healthy and post-stroke patients.

\section{Stroke rehabilitation considered in light of the International Classification of Functioning, Disability and Health (ICF)}

This conclusion that the efficacy of post-stroke rehabilitation interventions is improved when neurophysiological and pathological mechanisms are more specifically considered in their development is possibly a controversial conclusion when considered in light of the "biopsychosocial" model of health and disability. This model postulates that health and disability are best understood in terms of biological (physiology, biochemistry, pathology), psychological (thoughts, emotions, behaviours) and social (socio-economic, socio-environmental, cultural) factors, and that all aspects play a significant role. [35] This model is the basis for the International Classification of Functioning, Disability and Health (ICF) [34] mandated by the World Health Organisation in 2001 as the "framework for measuring health and disability at both individual and population levels" [36]. The proposition above that physiological, neural and pathological mechanisms should be more clearly considered in the development of rehabilitation interventions, emphasizes only the biological aspects of post-stroke disability. This does not suggest that the psychosocial aspects are less significant, rather an acknowledgement that psychosocial factors have traditionally had a central role in rehabilitation and physiotherapeutic diagnosis and treatment. [37-39] A focus on biological foundations and pathophysiology for effective treatment has however been lacking. The theoretical paradigms on which treatments have been developed, have often been based either on clinical observation and expert opinion, or on contemporary theories of neuromuscular control which do not comply with current knowledge. [40-43] Although in recent decades objective findings from basic neuroscience and clinical research have increasingly been integrated into theoretical paradigms of neurorehabilitation $[44,45]$ this has not been the case for all aspects of treatment. In particular the influence of walking aids on the neuromuscular control of gait in healthy subjects and stroke survivors has not been considered in this light. The possible influence on the recovery of balance and gait control has also not been investigated. 


\section{Walking aid research in stroke rehabilitation}

The vast majority of studies examining the influence of walking aids on gait in stroke subjects have investigated the immediate effects of canes on various biomechanical parameters, in laboratory conditions, using cross-sectional designs. [46-59] Outcomes include such parameters as gait velocity [58, 59]postural sway [50], muscle activation patterns $[53,58]$, kinematics and kinetics $[49,55-57,59]$ or balance and weight distribution [51]. The majority of these studies draw either neutral (no change) or positive conclusions about the influence of canes on gait parameters in stroke patients. For example Laufer et al. [52] and Maeda et al. [50] note that postural sway is reduced during cane walking, compared to walking with no cane, concluding that balance is improved. No discussion is presented regarding the possible mechanisms responsible for reduced sway, such as potentially increased activity in the arm to stabilize or increased base of support provided by the cane. Both of these possible mechanisms would induce balance reactions not usually seen in healthy individuals. This may have the long term effect of negatively impacting independent balance and therefore disability. Equally Buurke et al. [53] noted that muscle activity on the hemiplegic side and burst duration were reduced in hemiplegic patients when walking with a cane in the non-hemiplegic hand. The study concludes that canes should be considered in order to normalize hemiplegic muscle activation patterns during walking. The fact that muscle activity is reduced well below normal levels in healthy individuals [58] and the potential long-term effects thereof are not discussed. The majority of studies regarding the effect of walking aids in stroke rehabilitation are therefore limited to cane use, are cross-sectional in nature and do not consider potential long-term physiological or neural effects. The generally positive conclusions of these studies, without consideration of long term effects, are reflected in a recent Clinical Practice Guideline which states "in the opinion of the guideline development team, the use of walking-aids is beneficial to patients with a stroke in terms of safety, independence, and efficiency of walking, as well as confidence."[11] However a small number of prospective observational studies have been conducted. Hamzat et al. demonstrated that stroke patients using canes long-term had lower balance scores and less social participation than those without. [60] A similar study by Kim et al.[61] concluded that balance and falls efficacy are reduced in stroke patients who regularly use walking aids. Son et al. examined the effect of long-term cane use on the sensorimotor function of the non-hemiplegic, cane holding hand.[62] The study found significantly reduced sensorimotor function compared to non-cane using stroke patients, suggesting possible neural changes in the non-hemiplegic hand as it is used for support and less for skilled motor function.

The long term effects of canes on gait function and balance therefore remain unclear and opinions about prescription and use differ. Recommendations in Clinical Practice Guidelines do not necessarily reflect all current evidence, as prospective, observa- 
tional studies are not considered. Experimental evidence regarding long term effects is lacking however.

In a randomized controlled trial [63] Jung et al. used cane walking with bio-feedback as a treatment intervention for gait rehabilitation. Patients in the intervention group used pressure sensitive canes to provide support to enable them to walk, but were encouraged to reduce pressure on the cane via auditory feedback. Too much pressure caused an acoustic signal and the patients were asked to transfer weight onto the hemiplegic leg instead of the cane. In the control group patients walked with a cane as normal. The intervention group showed significantly better muscle activation levels and gait speed than the traditional cane using group. This study implies that the "normal" long-term use of canes, in which pressure is applied for support, reduces hemiplegic muscle activity and gait speed.

To our knowledge, no studies have considered the possible impact of walking aids on neuromuscular control or recovery of balance and gait in post-stroke subjects.

\section{Walking aids - prevalence and consequences}

Few statistics regarding the use of walking aids in the stroke population exist. Those that do are related to the American population. A "Medicare Current Beneficiary Survey" of 2001 indicated that of all chronic neurological patients assessed, 20.9\% used a cane and 23.2\% used a rollator.[64] A survey of mobility device use in the USA in 2000 conducted by the Institute for Health and Ageing from the University of California, estimated that nationwide 4.8 million people used a cane, 1.8 million a rollator, and 566,000 crutches. $22 \%$ of all cane users and $7.75 \%$ of all rollator users had cerebrovascular disease. [65] Similar figures were provided by West et al. who estimated that 4.8 million older adults (over 65) in the US used an assistive device to walk. [66] This study also indicated that device users were more likely to have experienced a recent fall, limited walking outside due to fear of falling or were unable to walk for 10 minutes outside without resting.

These figures indicate that cane and rollator use among stroke survivors in America is high. As no statistical data is available for other countries (that the author is aware of), making estimates about likely prescription rates and to what exact extent these figures can be generalised is difficult. However, there is no reason to assume that the European situation is different, and clinical experience supports this conclusion.

\section{Why may the influence of walking aids be important?}

Numerous factors which are known to be important for motor- learning and neural plasticity are present when stroke patients use walking aids. High intensity, task-specific training involving meaningful tasks for which patients are highly motivated has the largest influence on neural plasticity and motor learning. [67-69] Given that walking aids are 
often used for long periods of the day causing high repetition, for the important task of walking, the influence on balance and gait recovery may be significant, but adverse.

A greater understanding of the influence of walking aids would allow a more appropriate, evidence based prescription. When applied effectively, walking aids may be an inexpensive way of improving balance and gait, thus helping to reduce disability and the burden of disease.

\section{Aims of this thesis}

The aims of this thesis are:

- To review relevant aspects of the neural control of gait and the potential impact of walking aids on these control mechanisms in healthy and post-stroke individuals (chapters 2 and 3 ).

- To examine the immediate effects of various walking aids including canes, rollators, crutches, tape and an orthosis TheraTogs on gait parameters of healthy individuals, post-stroke subjects and individuals with non-neurological pathology (chapters 4, 5 and 6).

- To prospectively examine the influence and the effect size of different walking aids on the recovery of gait and balance in acute and chronic stroke patients (chapters 7 and 8).

- To generate knowledge which will contribute to evidence based recommendations for walking aid prescription in post-stroke patients (all chapters). 


\section{REFERENCES}

[1] Feigin VL, Forouzanfar MH, Krishnamurthi R, Mensah GA, Connor M, Bennett DA, et al. Global and regional burden of stroke during 1990-2010: findings from the Global Burden of Disease Study 2010. Lancet. 2014 Jan 18;383(9913):245-54.

[2] Feigin VL, Mensah GA, Norrving B, Murray CJ, Roth GA. Atlas of the Global Burden of Stroke (1990-2013): The GBD 2013 Study. Neuroepidemiology. 2015;45(3):230-6.

[3] Truelsen T, Heuschmann PU, Bonita R, Arjundas G, Dalal P, Damasceno A, et al. Standard method for developing stroke registers in low-income and middle-income countries: experiences from a feasibility study of a stepwise approach to stroke surveillance (STEPS Stroke). Lancet Neurol. 2007 Feb;6(2):134-9.

[4] worldstrokecampaign. www.worldstrokecampaign.org/learn/facts-and-figures.html. 2016.

[5] Scherbakov N, von Haehling S, Anker SD, Dirnagl U, Doehner W. Stroke induced Sarcopenia: muscle wasting and disability after stroke. Int J Cardiol. 2013 Dec 10;170(2):89-94.

[6] Go AS, Mozaffarian D, Roger VL, Benjamin EJ, Berry JD, Borden WB, et al. Heart disease and stroke statistics--2013 update: a report from the American Heart Association. Circulation. 2013 Jan 1;127(1):e6-e245.

[7] Bustamante A, Garcia-Berrocoso T, Rodriguez N, Llombart V, Ribo M, Molina C, et al. Ischemic stroke outcome: A review of the influence of post-stroke complications within the different scenarios of stroke care. Eur J Intern Med. 2015 Dec 23.

[8] Scherbakov N, Sandek A, Doehner W. Stroke-related sarcopenia: specific characteristics. J Am Med Dir Assoc. 2015 Apr;16(4):272-6.

[9] Michael KM, Allen JK, Macko RF. Reduced ambulatory activity after stroke: the role of balance, gait, and cardiovascular fitness. Arch Phys Med Rehabil. 2005 Aug;86(8):1552-6.

[10] Langhorne P, Bernhardt J, Kwakkel G. Stroke rehabilitation. Lancet. 2011 May 14;377(9778):1693-702.

[11] JM V, EEH vW, RPS vP, HIM H, MB R, PhJ vdW, et al. Royal Dutch Society for Physical Therapy - Clinical Practice Guideline for Physical Therapy in patients with stroke. 2014.

[12] Veerbeek JM, van Wegen E, van Peppen R, van der Wees PJ, Hendriks E, Rietberg M, et al. What is the evidence for physical therapy poststroke? A systematic review and meta-analysis. PLoS One. 2014;9(2):e87987.

[13] National Clinical Guideline C. National Institute for Health and Care Excellence: Clinical Guidelines. Stroke Rehabilitation: Long Term Rehabilitation After Stroke. London: Royal College of Physicians (UK)

National Clinical Guideline Centre. 2013.

[14] Weiss A, Suzuki T, Bean J, Fielding RA. High intensity strength training improves strength and functional performance after stroke. Am J Phys Med Rehabil. 2000 Jul-Aug;79(4):369-76; quiz 91-4.

[15] Stoller O, de Bruin ED, Knols RH, Hunt KJ. Effects of cardiovascular exercise early after stroke: systematic review and meta-analysis. BMC Neurol. 2012;12:45.

[16] Nudo RJ, Milliken GW, Jenkins WM, Merzenich MM. Use-dependent alterations of movement representations in primary motor cortex of adult squirrel monkeys. J Neurosci. 1996 Jan;16(2):785-807.

[17] Nudo RJ. Postinfarct cortical plasticity and behavioral recovery. Stroke. 2007 Feb;38(2 Suppl):840-5.

[18] Black JE, Isaacs KR, Anderson BJ, Alcantara AA, Greenough WT. Learning causes synaptogenesis, whereas motor activity causes angiogenesis, in cerebellar cortex of adult rats. Proc Natl Acad Sci U S A. 1990 Jul;:87(14):5568-72.

[19] Taub E. Movement in nonhuman primates deprived of somatosensory feedback. Exerc Sport Sci Rev. 1976;4:335-74.

[20] Taub E. The behavior-analytic origins of constraint-induced movement therapy: an example of behavioral neurorehabilitation. Behav Anal. 2012 Fall;35(2):155-78.

[21] Liepert J, Bauder H, Wolfgang HR, Miltner WH, Taub E, Weiller C. Treatment-induced cortical reorganization after stroke in humans. Stroke. 2000 Jun;31(6):1210-6.

[22] Wolf SL, Winstein CJ, Miller JP, Taub E, Uswatte G, Morris D, et al. Effect of constraint-induced movement therapy on upper extremity function 3 to 9 months after stroke: the EXCITE randomized clinical trial. JAMA. 2006 Nov;296(17):2095-104. 


\section{Chapter 1}

[23] Taub E, Uswatte G, Mark VW. The functional significance of cortical reorganization and the parallel development of $\mathrm{Cl}$ therapy. Front Hum Neurosci. 2014;8:396.

[24] Lalonde R, Botez MI. The cerebellum and learning processes in animals. Brain Res Brain Res Rev. 1990 Sep-Dec;15(3):325-32.

[25] Plautz EJ, Milliken GW, Nudo RJ. Effects of repetitive motor training on movement representations in adult squirrel monkeys: role of use versus learning. Neurobiol Learn Mem. 2000 Jul;74(1):27-55.

[26] Karni A, Meyer G, Rey-Hipolito C, Jezzard P, Adams MM, Turner R, et al. The acquisition of skilled motor performance: fast and slow experience-driven changes in primary motor cortex. Proc Natl Acad Sci U S A. 1998 Feb 3;95(3):861-8.

[27] Hubbard IJ, Parsons MW, Neilson C, Carey LM. Task-specific training: evidence for and translation to clinical practice. Occup Ther Int. 2009;16(3-4):175-89.

[28] Blennerhassett J, Dite W. Additional task-related practice improves mobility and upper limb function early after stroke: a randomised controlled trial. Aust J Physiother. 2004;50(4):219-24.

[29] Dean CM, Shepherd RB. Task-related training improves performance of seated reaching tasks after stroke. A randomized controlled trial. Stroke. 1997 Apr;28(4):722-8.

[30] Whitall J, McCombe Waller S, Silver KH, Macko RF. Repetitive bilateral arm training with rhythmic auditory cueing improves motor function in chronic hemiparetic stroke. Stroke. 2000 Oct;31(10):2390-5.

[31] Hubli M, Dietz V. The physiological basis of neurorehabilitation--locomotor training after spinal cord injury. J Neuroeng Rehabil. 2013;10:5.

[32] Dietz V. Body weight supported gait training: from laboratory to clinical setting. Brain Res Bull. 2009 Jan $15 ; 78(1):$ I-vi.

[33] Dundar U, Toktas H, Solak O, Ulasli AM, Eroglu S. A comparative study of conventional physiotherapy versus robotic training combined with physiotherapy in patients with stroke. Top Stroke Rehabil. 2014 Nov-Dec;21(6):453-61.

[34] Kim K, Lee S, Lee K. Effects of Progressive Body Weight Support Treadmill Forward and Backward Walking Training on Stroke Patients' Affected Side Lower Extremity's Walking Ability. J Phys Ther Sci. 2014 Dec;26(12):1923-7.

[35] Engel GL. The need for a new medical model: a challenge for biomedicine. Science. 1977 Apr 8;196(4286):129-36.

[36] Available from: http://www.who.int/classifications/icf/en/

[37] Mosey AC. An alternative: the biopsychosocial model. Am J Occup Ther. 1974 Mar;28(3):137-40.

[38] Samuels BM, Ritchie DM. Some social and psychological aspects of rheumatoid arthritis. Physiotherapy. 1970 Sep;56(9):404-8.

[39] Suchett-Kaye Al, Sarkar U, Elkan G, Waring M. Physical, mental and social assessment of elderly patients suffering from cerebrovascular accident with special reference to rehabilitation. Gerontol Clin (Basel). 1971;13(4):192-206.

[40] B B. Adult Hemiplegia Evaluation and Treatment: Heinemann Medical 1978

[41] Semans S. The Bobath concept in treatment of neurological disorders; a neuro-developmental treatment. Am J Phys Med. 1967 Feb;46(1):732-88.

[42] Stockmeyer SA. An interpretation of the approach of Rood to the treatment of neuromuscular dysfunction. Am J Phys Med. 1967 Feb;46(1):900-61.

[43] Brunnstrom S. Motor testing procedures in hemiplegia: based on sequential recovery stages. Phys Ther. 1966 Apr;46(4):357-75.

[44] Woollacott MH, Shumway-Cook A. Changes in posture control across the life span--a systems approach. Phys Ther. 1990 Dec;70(12):799-807.

[45] Shepherd R, Carr J. Reflections on physiotherapy and the emerging science of movement rehabilitation. Aust J Physiother. 1994;40s:39-47.

[46] Milczarek JJ, Kirby RL, Harrison ER, MacLeod DA. Standard and four-footed canes: their effect on the standing balance of patients with hemiparesis. Arch Phys Med Rehabil. 1993 Mar;74(3):281-5. 
[47] Tyson SF, Rogerson L. Assistive walking devices in nonambulant patients undergoing rehabilitation after stroke: the effects on functional mobility, walking impairments, and patients' opinion. Arch Phys Med Rehabil. 2009 Mar;90(3):475-9.

[48] Tyson SF. The support taken through walking aids during hemiplegic gait. Clin Rehabil. 1998 Oct;12(5):395-401.

[49] Chen CL, Chen HC, Wong MK, Tang FT, Chen RS. Temporal stride and force analysis of cane-assisted gait in people with hemiplegic stroke. Arch Phys Med Rehabil. 2001 Jan;82(1):43-8.

[50] Maeda A, Nakamura K, Higuchi S, Yuasa T, Motohashi Y. Postural sway during cane use by patients with stroke. Am J Phys Med Rehabil. 2001 Dec;80(12):903-8.

[51] Laufer Y. Effects of one-point and four-point canes on balance and weight distribution in patients with hemiparesis. Clin Rehabil. 2002 Mar;16(2):141-8.

[52] Laufer $Y$. The effect of walking aids on balance and weight-bearing patterns of patients with hemiparesis in various stance positions. Phys Ther. 2003 Feb;83(2):112-22.

[53] Buurke JH, Hermens HJ, Erren-Wolters CV, Nene AV. The effect of walking aids on muscle activation patterns during walking in stroke patients. Gait Posture. 2005 Oct;22(2):164-70.

[54] Beauchamp MK, Skrela M, Southmayd D, Trick J, Kessel MV, Brunton K, et al. Immediate effects of cane use on gait symmetry in individuals with subacute stroke. Physiother Can. 2009 Summer;61(3):154-60.

[55] Polese JC, Teixeira-Salmela LF, Nascimento LR, Faria CD, Kirkwood RN, Laurentino GC, et al. The effects of walking sticks on gait kinematics and kinetics with chronic stroke survivors. Clin Biomech (Bristol, Avon). 2012 Feb;27(2):131-7.

[56] Kuan TS, Tsou JY, Su FC. Hemiplegic gait of stroke patients: the effect of using a cane. Arch Phys Med Rehabil. 1999 Jul;80(7):777-84.

[57] Tyson SF. Trunk kinematics in hemiplegic gait and the effect of walking aids. Clin Rehabil. 1999 Aug;13(4):295-300.

[58] Maguire C, Sieben JM, Frank M, Romkes J. Hip abductor control in walking following stroke -- the immediate effect of canes, taping and TheraTogs on gait. Clin Rehabil. 2010 Jan;24(1):37-45.

[59] Allet L, Leemann B, Guyen E, Murphy L, Monnin D, Herrmann FR, et al. Effect of different walking aids on walking capacity of patients with poststroke hemiparesis. Arch Phys Med Rehabil. 2009 Aug;90(8):1408-13.

[60] Hamzat TK, Kobiri A. Effects of walking with a cane on balance and social participation among community-dwelling post-stroke individuals. Eur J Phys Rehabil Med. 2008 Jun;44(2):121-6.

[61] Kim O, Kim JH. Falls and Use of Assistive Devices in Stroke Patients with Hemiparesis: Association with Balance Ability and Fall Efficacy. Rehabil Nurs. 2015 Jul-Aug;40(4):267-74.

[62] Son SM, Kwon JW, Nam SH, Lee NK, Kim K, Kim CS. Adverse effects of motor-related symptoms on the ipsilateral upper limb according to long-term cane usage. NeuroRehabilitation. 2012;31(2):137-41.

[63] Jung K, Kim Y, Cha Y, In TS, Hur YG, Chung Y. Effects of gait training with a cane and an augmented pressure sensor for enhancement of weight bearing over the affected lower limb in patients with stroke: a randomized controlled pilot study. Clin Rehabil. 2015 Feb;29(2):135-42.

[64] Wolff JL, Agree EM, Kasper JD. Wheelchairs, walkers, and canes: what does Medicare pay for, and who benefits? Health Aff (Millwood). 2005 Jul-Aug;24(4):1140-9.

[65] S K, T K, MP L. Mobility Device in the United States. Institue for Health and Ageing, University of California, San Francisco, USA. 2000.

[66] West BA, Bhat G, Stevens J, Bergen G. Assistive device use and mobility-related factors among adults aged $>$ /=65years. J Safety Res. 2015 Dec;55:147-50.

[67] Winstein C, Lewthwaite R, Blanton SR, Wolf LB, Wishart L. Infusing motor learning research into neurorehabilitation practice: a historical perspective with case exemplar from the accelerated skill acquisition program. J Neurol Phys Ther. 2014 Jul;38(3):190-200.

[68] Nudo RJ. Adaptive plasticity in motor cortex: implications for rehabilitation after brain injury. J Rehabil Med. 2003 May(41 Suppl):7-10.

[69] Kleim JA, Jones TA. Principles of experience-dependent neural plasticity: implications for rehabilitation after brain damage. J Speech Lang Hear Res. 2008 Feb;51(1):S225-39. 



\section{Chapter}

The influence of walking aids on the plasticity of Spinal Interneuronal Networks, Central Pattern Generators and the recovery of gait post-stroke. A review of the literature.

Authors

Clare C. Maguire, Judith M. Sieben, Robert. A. de Bie

Submitted for publication. 


\section{ABSTRACT}

Fundamental neuroscience has been the foundation for the development of some effective treatments in stroke rehabilitation such as Constrained Induced Movement Therapy. However, many facets of gait rehabilitation have not been adequately considered in this context and remain based on low level clinical evidence or expert opinion. In particular the use of walking aids including canes and rollators (wheeled walkers), although widely used and often for long periods, have not been extensively investigated in terms of impact on the neural and neuromuscular mechanisms controlling gait and balance. Studies have primarily been limited to the immediate effects on kinetic, kinematic or physiological outcomes. In this article we review the structure and function of spinal interneuronal networks and central pattern generators in healthy and post-stroke patients. The influence of afferent inputs on these structures is discussed. Recent evidence demonstrating spinal post-stroke plasticity is presented, relevance for gait recovery and the possible influence of walking aids on these mechanisms are considered. Potential consequences for rehabilitation and areas requiring future research are debated.

Keywords: cerebrovascular stroke, walking, assistive devices, Central Pattern Generators, neuronal plasticity. 


\section{INTRODUCTION}

The most effective treatments for post-stroke rehabilitation, when considering Clinical Practice Guidelines and systematic reviews, are those whose development was based on sound knowledge of basic neuroscience.[1, 2] Despite this, information from basic science is not routinely considered in many aspects of gait rehabilitation. Clinical practice is often based on low level evidence or expert opinion.[3-5] Walking aids, including canes and rollators, although widely used and often for long periods, have not been investigated regarding impact on neural and neuromuscular mechanisms. To date, studies have primarily been limited to the immediate effects on kinetic, kinematic or physiological outcomes in cross-sectional studies. [6, 7]

We review spinal neuronal networks (SINs), Central Pattern Generators (CPGs) and the influence of afferent feedback on these structures in healthy and post-stroke subjects. We discuss possible influences of walking aids on the control and recovery of these systems and gait function, the potential consequences for rehabilitation and areas requiring future research.

\section{A. Spinal interneurons}

Neuronal networks are groups of neurones, each with potentially different input and output relationships and intrinsic properties, which work co-operatively and in parallel.[8] They are distributed throughout the central nervous system, forming for example cortical, vestibular, cerebellar and spinal neural networks.

Spinal interneuronal networks, SINs, are thought to represent the functional link translating higher centre commands and integrating peripheral feedback to achieve orchestrated output at the motor neurones (MNs).[9] This enables coordinated muscle activity to achieve motor goals. SINs are involved in all levels of movement control, from simple reflex responses to complex voluntary tasks.[10] They play an important role in turning the "space based" representation of movement in the motor cortex (e.g. hand to mouth)[11] into specific muscle activity via MNs. They seem to be decisive in reducing the redundancy inherent in cortical movement maps and generating specific patterns of activity.[12]

Locomotor CPGs represent one form of hereditary, stereotyped although flexible SIN.[9]The function of CPGs in gait control and the relevance for rehabilitation has been considered primarily for patients with spinal cord injury(SCI).[13] The importance for stroke rehabilitation has been sparsely considered[14, 15] and to our knowledge not at all for the prescription of walking aids.

\section{B. Structure of SINS}

This paper uses a functional classification of SINs.[16] 
SINs consist of 1. Input neurones from various sources including supraspinal, peripheral afferents and interneurons (INs) from other SINs. 2. Numerous types of INs which process and integrate the inputs. 3. Target output neurones which may be MNs, the interneurons of other SINs, or supraspinal structures. [17]

\section{Spinal interneuronal network (SIN)}

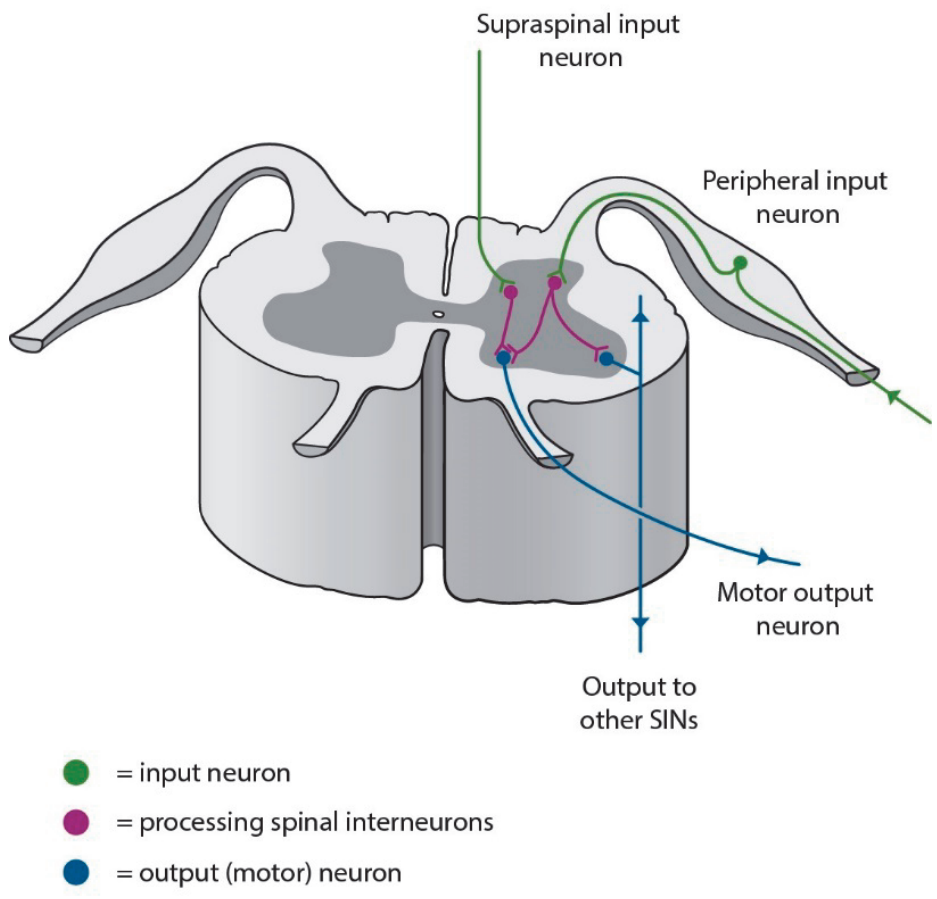

Figure 1. Schematic representation of a Spinal interneuronal network (SIN)

\section{Input-neurons}

Supraspinal input neurones include cortical, vestibular, reticular, cerebellar and rubrospinal sources. Peripheral inputs arise from muscle, tendon, cutaneous, joint, osseous and pain fibres.[18]

The proportion of inputs from different sources varies depending on the spinal segment. MNs directing hand and finger movement receive the most direct corticospinal inputs, the proportion is nevertheless estimated to be $10 \%$ or less. Tracing of corticospinal pathways suggests that most fibres terminate directly onto INs rather than MNs [19], emphasising the integrative importance of these structures. The synaptic strength 
of cortical-MN connections is also weaker than cortical-IN and IN-MN connections. This supports the hypothesis that INs participate in the process of translating cortical commands into muscle activity.

\section{Divergence of input}

Afferent neurons form divergent connections with large numbers of INs.[18] This simultaneous delivery of information to multiple effectors supports a holistic and coordinated response.

\section{Convergence of input}

SINs simultaneously receive input from many different sources.[16] Final output will depend on the weighting and integration of received signals. This integrative function appears to be the main task of interneurons.[20]

\section{Interneurons}

\section{Intra-limb control}

\section{Inhibition}

\section{Reciprocal inhibition.}

Reciprocal inhibition refers to the intra-limb, inter-muscular inhibition of antagonist musculature during agonistic activation.

\section{Presynaptic reciprocal inhibition-stretch reflex}

For example, due to the stretch reflex a sudden stretch of an extensor muscle triggers its reflex contraction thereby stretching the antagonistic flexor. This could trigger a stretch reflex in the flexor muscle, potentially creating a series of agonist-antagonist reflex contractions. To prevent this, $1 \mathrm{~b}$ afferents from the antagonistic muscles are simultaneously and "reciprocally" inhibited during the agonist reflex contraction. This inhibition prevents the antagonistic MNs from receiving the information that a stretch has occurred and therefore from firing.[21] This is an example of pre-synaptic inhibition and is mediated by ipsilateral spinal INs.

\section{Postsynaptic reciprocal inhibition-gait control}

Reciprocal inhibition during walking is important for coordinated limb control, although mediated by a different mechanism than the stretch reflex. Agonistic 1a afferents synapse onto 1a INs which use post-synaptic rather than pre-synaptic inhibition. These 1a INs synapse directly onto the soma of antagonistic MNs therefore inhibiting the MN itself and not it's afferent.[9] These antagonistic inhibitory neurons are activated during 
all agonistic activations even without a stretch reflex. If 1 a INs are blocked, agonistantagonist co-contraction occurs.[22] These networks are primarily ipsilateral as they remain intact in the hemi cord[23]. Reciprocal inhibition therefore provides a principle mechanism by which muscles can be linked and coordinated.[18]

\section{Reciprocal inhibition - inhibitory input to antagonist for inter-muscular, intra-limb control}

Stretch Reflex - presynaptic inhibition - prevents a series of agonistic - antagonistic reflex contractions.
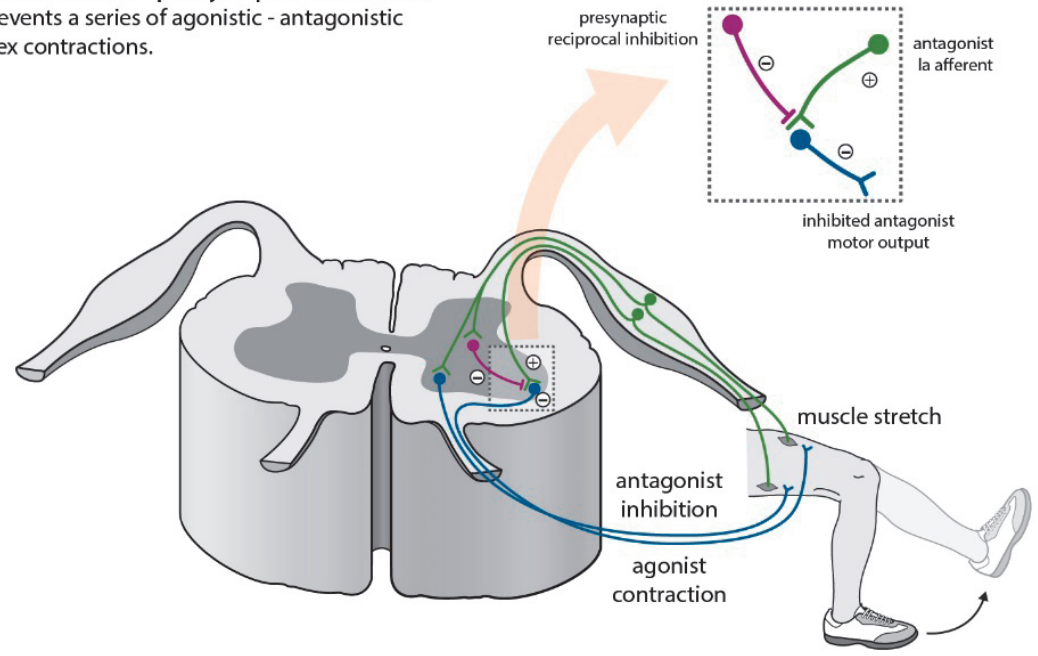

During gait control - postsynaptic inhibition - occurs even in the absence of a stretch reflex and prevents intra-limb co-contraction postsynaptic
eciprocal inhibition reciprocal inhibition
during gait
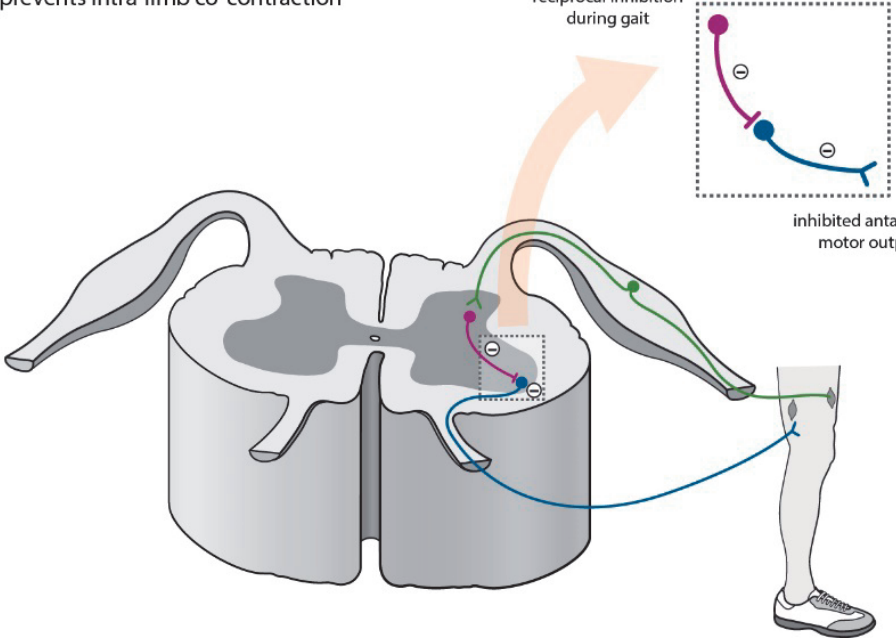

Figure 2. Schematic representation of pre-synaptic and post-synaptic reciprocal inhibition - $=1$ a sensory afferent (muscle spindle)

= processing spinal interneuron

= output (motor) neuron 


\section{Non-reciprocal inhibition}

Animal experiments demonstrate that non-reciprocal inhibition is governed by $1 \mathrm{~b}$ INs. These are activated by muscle and cutaneous afferents, joint receptors and cortical inputs and can project to both agonistic and antagonistic muscle groups.[24] This enables intra-limb muscular inhibition providing a protective mechanism and allowing supra-spinal influence of muscular activity levels.

\section{Non-reciprocal inhibition - Autogenic inhibition (Golgi tendon reflex)}

This reflex is the opposite of the stretch reflex. $1 \mathrm{~b}$ afferent fibres from Golgi Tendon organs fire during muscle contractions signalling tension in the muscle. The fibres synapse onto $1 \mathrm{~b}$ inhibitory INs of their own MN. If signals from $1 \mathrm{~b}$ afferents indicate that muscle tension is high, then $1 \mathrm{~b}$ INs increase firing rate thereby increasing inhibition at their homologous MN. This reduces activity thus having a protective effect.[22, 24]

This circuit operates not only as a protective reflex, but also at lower levels of muscle tension. The inhibition of MNs via $1 \mathrm{~b}$ INs can be reduced or increased, depending on the requirements of the motor task.[24] As the $1 \mathrm{~b}$ INs receive multiple inputs, the final output, whether a reduction or an increase in inhibition, will depend on the integration of incoming information. In the stance phase of gait, despite increased extensor muscle activity which will increase $1 \mathrm{~b}$ afferent signals, a further increase not a decrease in extensor activity occurs. $[25,26]$ The integration of inputs from various sources e.g. joint load receptors, influences final output. SIN output therefore adapts depending on motor goal.[27]

\section{Non-reciprocal inhibition -Recurrent inhibition}

Renshaw cells(RCs) are inhibitory cells located ventrally in the spinal cord. During firing of MNs, the signal is also forwarded, by axon collaterals from the MN, to the Renshaw cell. This stimulates the inhibitory output of the RC which makes synaptic connections to the stimulating $\mathrm{MN}$, to several other synergistic MNs in adjacent segments and to $1 \mathrm{a}$ inhibitory neurones.[18, 28]. This creates a negative feedback loop, in which increasing activity of the MN increases signals to the inhibitory RC, thus reducing the MN output. This process plays an important role in the termination of $\mathrm{MN}$ burst during locomotion. Connections to the $1 \mathrm{a}$ inhibitory neurones may regulate the strength of reciprocal inhibition.[18, 28]. Connections to other local MNs causes a generalised, not muscle specific, inhibitory effect. These processes are termed recurrent inhibition.[29] Recurrent inhibition is strongest among proximal postural muscles during postural control and locomotion. $[18,28]$ In contrast, recurrent inhibition is weak or absent in MN pools concerned with precise, distal voluntary movement.[22] This may reflect the diffuse nature of $\mathrm{RC}$ inhibition which is adequate for postural control but not for the exactitude required for skilled voluntary movement. 


\section{Chapter 2}

Animal models demonstrate that RCs also receive input from descending pathways, commissural INs (from the contralateral side) and during walking from the ipsilateral locomotor networks.[28]

\section{Non-reciprocal inhibition - inhibitory input to own MN for intra-muscular tension control}

\section{Autogenic inhibition (Golgi tendon reflex)}

- Increasing agonistic muscle tension

Increases activity in $1 \mathrm{~b}$ inhibitory INs thus inhibiting

own motor output

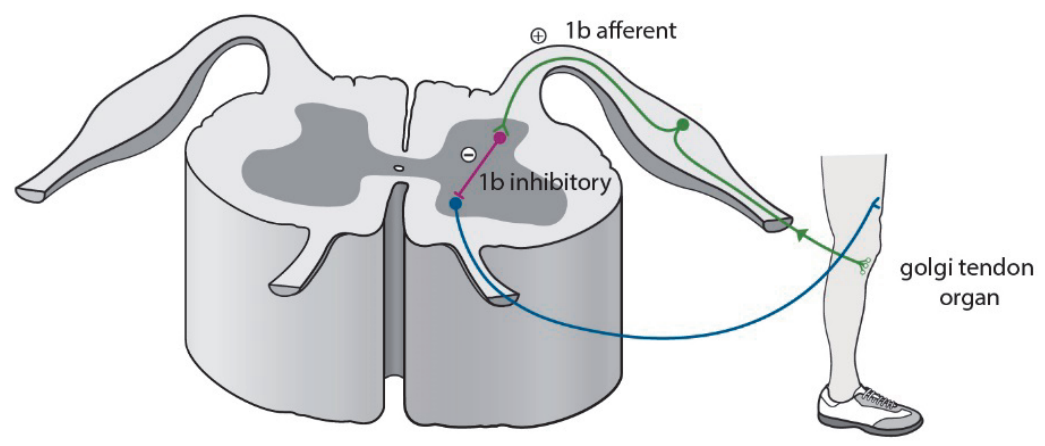

Recurrent inhibition (via Renshaw Cells)

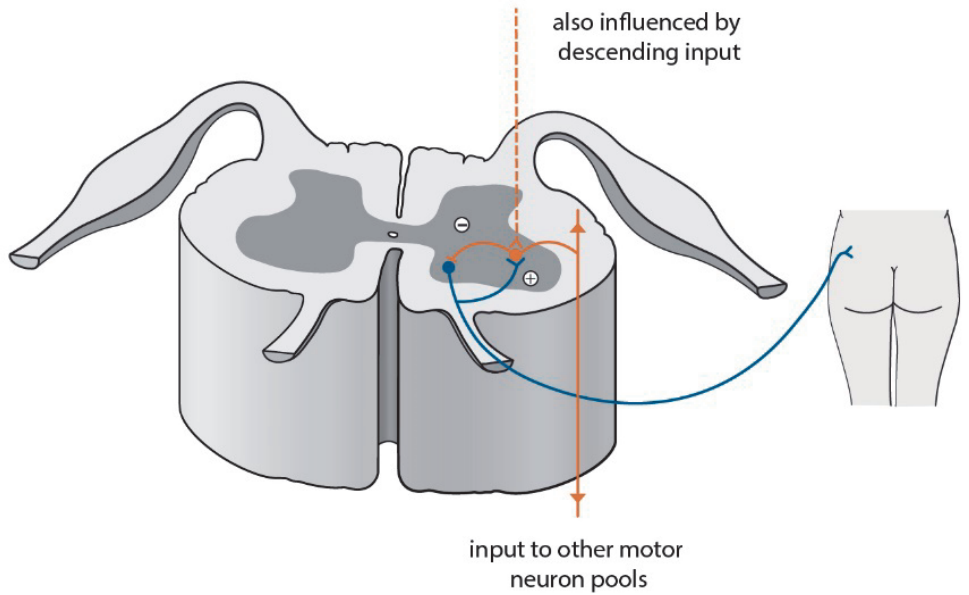

Figure 3. Schematic representation of two types of non-reciprocal inhibition - autogenic via Golgi tendon organ and recurrent via Renshaw Cells

$=1 \mathrm{~b}$ sensory afferent (golgi)

= processing spinal interneuron

= output (motor) neuron

$=$ Renshaw cell 


\section{Excitation}

\section{Afferent Inputs}

Direct connections from group 1a and II muscle afferents onto MNs provide excitatory inputs. The strength of these connections varies depending on the motor task and the afferent fibre. The strongest connections arise from 1a afferents during postural tasks of antigravity muscles.[22]. Evidence shows convergent excitation from cutaneous, joint and group III muscle afferents. Excitatory inputs from supraspinal structures selectively increase activity in SINs. Signals from the "brainstem command centres" such as the Mesencephalic Locomotor Region which is involved in the initiation of gait and control of muscle tone, as well as direct cortico-spinal excitatory inputs.[30]

\section{Excitatory spinal INs}

In animal studies at least four types of INs have been shown to release the excitatory neurotransmitter glutamate and target MNs as well as commissural INs (CINs). The influence on commissural INs suggests an important role for left-right coordination. They also appear to be involved in rhythm generation during gait through rhythmic and repetitive firing.[31]

\section{Inter-limb coordination}

Inter-limb coordination is an important aspect of gait and balance control. For example initiation of swing phase is dependent on ipsilateral signals indicating the end of stance phase and contralateral signals indicating stance. Equally, if one leg is displaced during standing or walking a coordinated bilateral response occurs.[32] This is achieved by both CINs[30] and supraspinal inputs involving the corpus callosum.[14]. A further example is the polysynaptic flexor withdrawl reflex.[33]

\section{Commissural Interneurones}

"Commissural interneurons" (CINs) have been identified in animal studies and can have axonal projections which are both intra- and intersegmental (level of each spinal nerve), descending and ascending.[9] CINs are widely interconnected thus acting as building blocks of complex networks.[17] They integrate information from supraspinal and peripheral sources and provide direct excitation, direct and indirect inhibition to ipsi- and contralateral MNs. 


\section{Commissural interneurons (CINS) involved in inter-limb muscular coordination (identified in animal studies)}

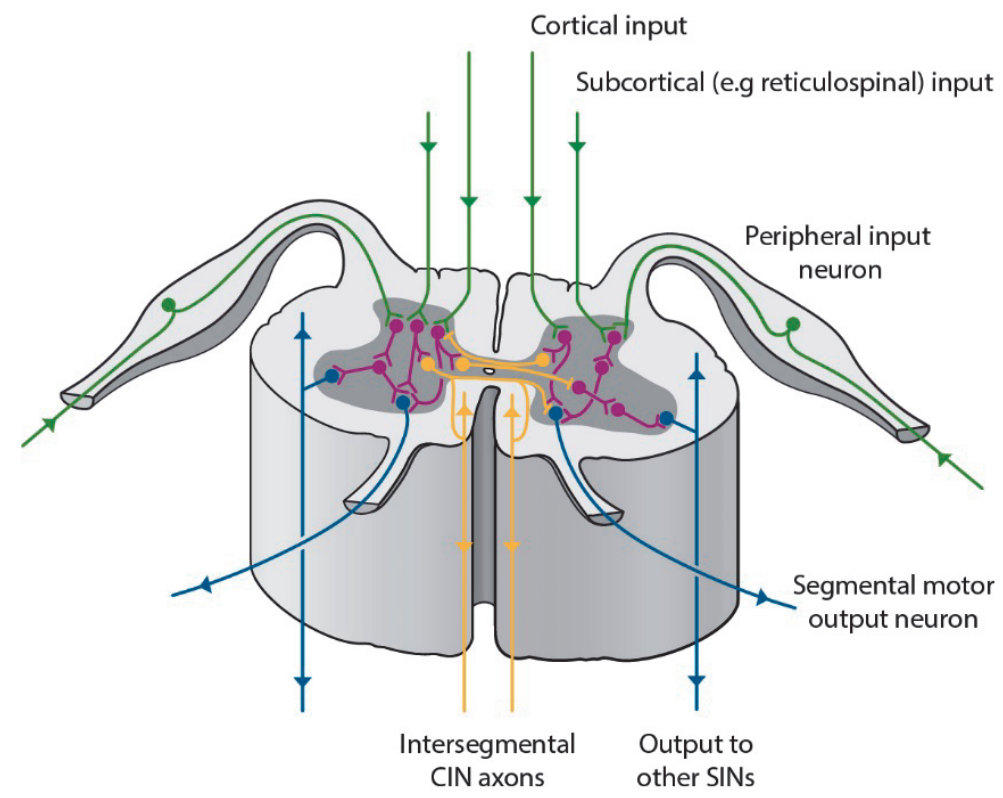

Figure 4. Schematic representation of Commissural interneurons (CINs)

\section{Alternating and synchronous limb movement}

A dual inhibitory system is involved in left-right alternating patterns (anti-phase movement in walking) and synchronous bilateral patterns (in-phase movement such as jumping with two legs).[21] During alternating movement, ipsilateral activation and contralateral inhibition occur simultaneously via a direct inhibitory pathway, mediated by inhibitory GABAergic INs, which project onto contralateral motor neurones and an indirect pathway provided by excitatory glutametergic INs, which synapse onto contralateral inhibitory Renshaw cells.

Excitatory CINs can simultaneously synapse onto MNs and Renshaw cells. This is thought to be important during synchronous activity when both limbs perform the same task.[21] 


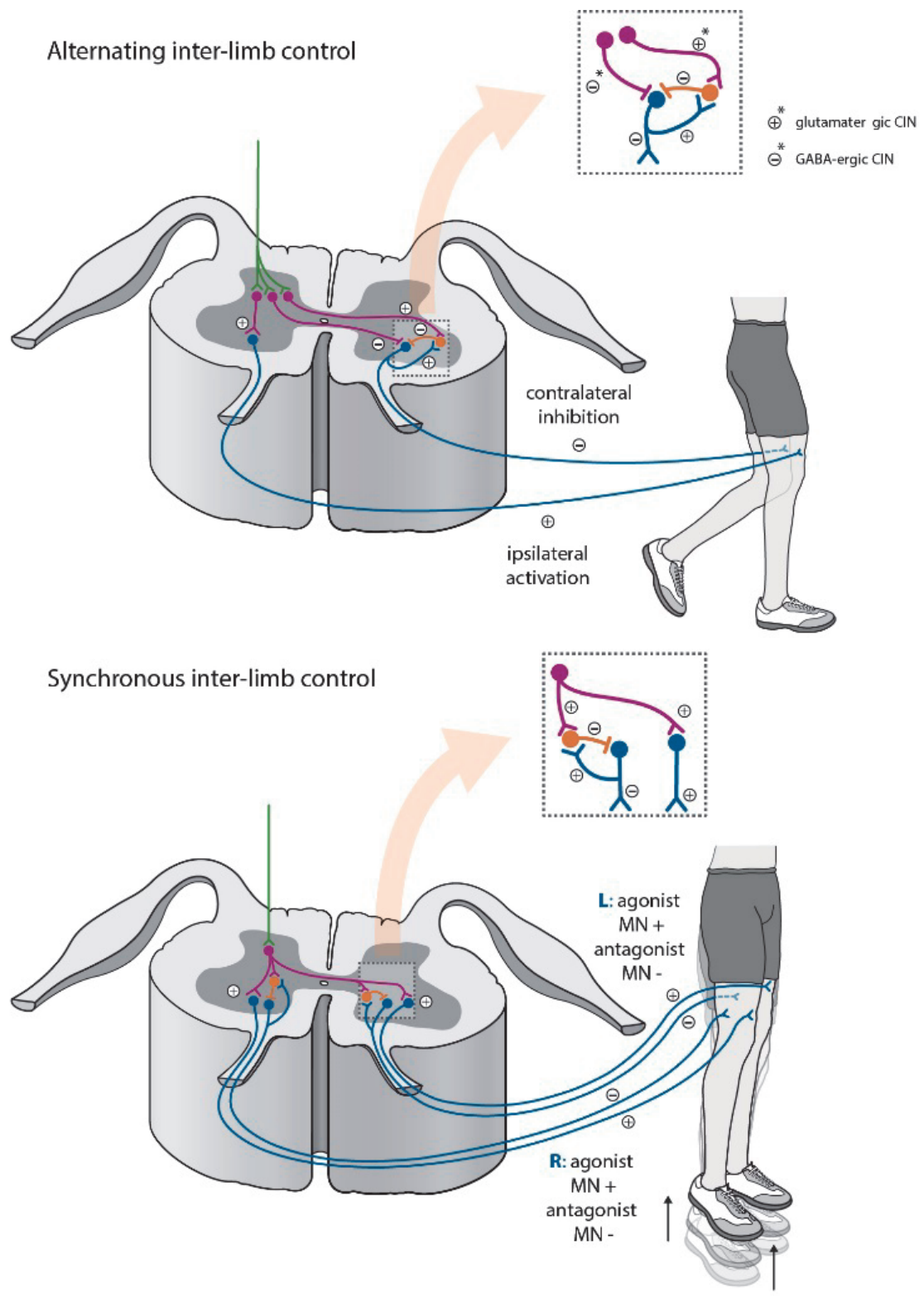

Figure 5 Schematic representation of alternating and synchronous inter-limb control
= descending input
$=$ CINS $^{*}$
= ipsi- and contralateral motor output
= inhibitory Renshaw cell 


\section{Afferent input}

Direct connections exist between CINs and higher centres. Reticulospinal and vestibulospinal connections illustrate that CINs are involved in postural adjustments. Inputs from the pyramidal tract and the mesencephalic locomotor region(MLR) indicate involvement in gait initiation[17] and with the cortex, in the earliest stages of movement preparation.[34] Input from fastigial neurons from the cerebellum indicates involvement in feedforward adaptation and motor learning.[17] Connections from muscle afferents indicate integration of peripheral afferent signals.

Studies demonstrate that supraspinal inputs are essential for the correct functioning of CINs and lower limb coupling.[35] Passive facilitation of walking in one leg with a Dynamic-Gait-Orthosis induced muscle activity in the opposite (still) leg in healthy subjects but not in $\mathrm{SCl}$ subjects.

\section{SIN Output}

\section{To motor neurones}

"The majority of inputs to spinal MNs originate from premotor SINs."[16] Motor neurones are large cells, each one receiving between 50-150K synaptic inputs, approximately $40 \%$ of which are excitatory, $60 \%$ inhibitory.[12] They receive signals from diverse sources including local segmental afferent information and remote inputs from distant segments and descending pathways.

\section{To ascending fibres}

Ascending fibres to supraspinal centres contribute to the monitoring of motor output, inter-limb coordination and to the perception of limb position.[24]

\section{To other SINs}

Output to other SINs enables connectivity and coordinated control.

\section{B. Locomotor CPGs}

\section{CPGs hereditary, stereotyped but flexible SINs}

CPG's have been extensively investigated in animals and indirectly in humans.[35-39]. They are genetically determined networks whose structure and function are modulated through experience.[40] CPGs in the brainstem control breathing and swallowing and spinal cord CPGs control walking.[41] Spinal CPGs influence three aspects of locomotion 1. Rhythm and cycle 2.Intra-limb agonist-antagonist coordination 3.Inter-limb coordination[39]. 
The basic structure of CPGs consists of agonist and antagonist "half-centres", or task-specific sub-populations of INs, which are mutually inhibitory.[42] These are mediated by reciprocal inhibition through 1 a INs. Animal studies show that half-centres exist for specific joints and are situated on each side of the spinal cord for each limb.[39]. It is suggested that half-centres, work together to coordinate movement across joints.[39] This organisation enables flexible combinations of motor actions thus facilitating task specific recruitment of muscle activity. Different models describe how this coordination occurs. One commonly cited model[43] suggests that multi-joint coordination is controlled via SINs connecting joint specific half-centres. All forms of bipedal locomotion in humans, from very slow walking to fast running, appear to be controlled by the flexible combination of four to five CPGs. [44] The strength of excitation and inhibition at each unit is modulated according to the motor task thus allowing flexible and context dependent motor output.[45]

Half-centres are further subdivided. One part determines timing and muscle activation during stance and the transition to swing. The other during swing phase.[45]

Although the basic pattern and rhythm of walking can be produced by CPGs alone, for the skilled and context dependent adaptations of gait required in daily activity, input from supraspinal centres and peripheral afferents is crucial. These inputs determine which components of CPGs are combined to achieve a motor goal thus influencing the timing and level of muscle activation.

\section{Upper and lower limb co-ordination}

In some situations e.g. swimming, coordination between all four limbs is required. CINs connecting the cervical and lumbar enlargements of the spinal cord appear to be responsible for upper and lower limb co-ordination. $[32,46]$ However, unique to humans, due to bipedal stance and skilled hand movements, is the ability to disengage the upper limbs from four limb CPG control when skilled hand movements are required. Dietz et al. have demonstrated that when the lower extremities are stimulated, EMG responses are monitored in the arms when the subject is walking but not when sitting and writing.[47] This implies a "task-dependent neuronal coupling " between upper and lower limbs in which spinal circuits can enable four-limb co-ordination or can be gated via cortical activity to free arm control for skilled movements.[32, 48] This implies that tasks requiring upper limb activity outside the cyclical control of spinal CPGs require increased cortical input. fMRI studies indicate that the cortical supplementary motor area (SMA) is involved in this control.[49]

\section{C.Influence of peripheral afferents on gait}

Studies in individuals with complete $\mathrm{SCl}$ demonstrate that stepping patterns can be driven solely by information from peripheral sensory afferents.[50] Information from 
load receptors, hip joint movement, cutaneous and muscular proprioceptors appear to be particularly influential in determining final motor output.[30, 35, 38].

Dietz et al.[35] demonstrated that passive physiological gait movements alone, without joint loading (facilitated with a Driven-Gait-Orthosis) did not induce muscle activity in healthy or $\mathrm{SCl}$ subjects. These results confirm earlier findings which show that load receptors are essential to trigger extensor muscle activity during walking in humans.[26] Flexor activity appears to be less influenced by load receptors, probably due to more central control during walking.[35, 37].

This study[35] also showed that isolated, loaded hip movement without movement at the knees or ankles, induced normal walking activity patterns in healthy and SCl subjects. Isolated loaded knee or ankle movement did not induce walking patterns. Therefore, sensory feedback from both joint load and hip movement receptors appears to be decisive to facilitate normal CPG activity. These findings were confirmed by Pearson et al. who found that preventing hip extension can inhibit the transition to swing phase.[51] Stretch at the hip flexor musculature signaling the end of stance phase may influence the initiation of swing phase. As CPGs are functionally divided into parts that control i) stance and transition to swing and ii) swing phase,[45] it appears that joint load, hip movement and hip flexor stretch receptors are important in the stance and transition phase. The swing phase appears to be more influenced by central, cortical control.[52]

Muscle afferents also influence activity in SINs. Swing phase is not initiated until intra-limb plantar flexor activity is low and the muscle is unloaded[53].

Sinkjaer et al. showed that during stance phase, unloading the ankle extensors reduced soleus activity by $50 \%$ in early and mid-stance.[54] McCrea et al.[55] showed in animal studies that depending on timing, stimulation of ankle extensor afferents could cause "a premature initiation of the extension phase, entrain the locomotor stepping frequency, alter the period of the flexion and extension phases or increase extensor motoneuron activity."

Cutaneous afferents also impact CPG activity and are important for correct foot placement and obstacle avoidance.[56,57] Cutaneous afferents stimulated in cats in opposite phases of gait induce opposite postural reactions. If the cutaneous plantarflexor paw surface is stimulated during swing phase, flexion activity is increased suggesting obstacle avoidance strategies. If the same surface is stimulated during stance phase, extensor activity is increased.[30] Drew et al.(2004) suggest this response may be due to cutaneous inputs being relayed to the reticular formation in the brainstem. This input triggers reticulospinal neurones which converge onto spinal CPGs and "will tend to re-inforce activity in muscles that are already active".[58]

Speed of displacement, signalled from numerous peripheral afferents simultaneously, influences the activity level of synergistic muscle responses.[59]

The above points illustrate several important roles of sensory feedback. Firstly to help direct the activity of the motor neurones and secondly to contribute to adaptive 
and corrective responses in challenging situations or following perturbation. [36]. Thirdly sensory receptors send error signals in the ascending tracts to inform supraspinal structures to what extent completed movements resemble intended movements. This is important for adjustment of future movements i.e. for fine motor tuning and motor learning.[36] It has been suggested that "The rhythm and pattern are transmitted back to the supraspinal structures by the spinothalamic, - reticular, and -cerebellar tracts, so that the supraspinal structures monitor all events in the spinal cord."[30]

\section{$\underline{\text { Important afferent input in healthy gait for normal CPG activity }}$}

\section{Four limb CPG coupling to reduce cognitive demands}

2. Normal hip joint loading

3. Hip movement particulary hip extension to trigger transistion to swing phase

4. Normal muscle activity levels and loading

5. Cutaneous inpuls particulary to signal when adaptations are necessary for obstacle avoidance or on uneven surfaces

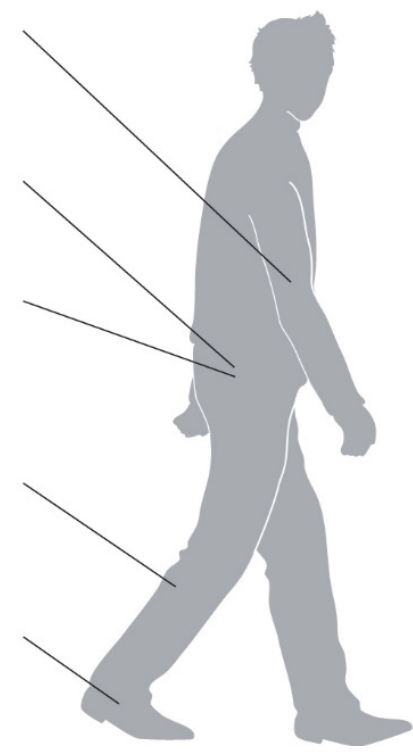

Figure 6. Afferent input necessary for normal CPG activity in healthy gait

\section{Modification and co-operation between SINs as motor tasks change}

Studies indicate that the functions of SINs adapt depending on the motor goal.[20, 60, 61] For example following position change from supine to standing the soleus stretch reflex decreases by $10-15 \%$ although there is a general increase in background soleus EMG.[62] It appears that spinal circuits have simultaneously enabled an increase in activity and a decrease in reactivity. This could be achieved by increased presynaptic inhibition of group I muscle afferents together with a direct increase in excitation of the MN. Optimal movement control therefore requires the ability to adapt interneuronal activity between postures and movements. 


\section{SINs may facilitate change between feedforward and feedback control}

Peripheral afferent information can be blocked due to pre-synaptic inhibition from GABA-ergic INs whilst at the same time excitability of the post-synaptic cell (e.g.MN) is unaffected. This reduction of afferent information has been shown to take place directly before movement onset[63] and the command originates in the SMA.[64]. This allows changes in the weighting of descending and peripheral inputs influencing MNs. Motor control can switch from feedforward, supraspinal control during movement onset to more feedback, peripherally influenced on-line control during movement.[12]

These adaptations further demonstrate the functional flexibility of spinal cord circuitry necessary for movement control.

\section{Relevance for use of walking aids post stroke}

The decisive influence of sensory input on motor output has been demonstrated, thus offering a mechanism by which therapy can influence SIN and CPG activity. These influences have the potential to be beneficial or harmful depending on method and timing of delivery.

\section{Hip joint loading}

Limb loading, particularly of the hip joint, is essential for normal CPG activity and transition from stance to swing phase. This has been used to develop effective treatments for $\mathrm{SCl}$ patients. "Load- and hip-joint-related afferent input seems to be of crucial importance for both the generation of a locomotor pattern and the effectiveness of the training."[65] Additionally, neurologically intact humans and primates returning from space exhibit clonus in the lower extremities. This suggests that clonus is highly influenced by loss of joint load, even when supraspinal input remains intact.[66]

Walking with canes in the contralateral, non-hemiplegic hand in stroke patients unloads the opposite, hemiplegic hip.[67, 68] When walking with rollators, muscle activity is reduced in the lower extremities and is compensated for by increased use of the arms, suggesting that the hips are unloaded during rollator walking.[69] These devices reduce normal hip loading, therefore altering afferent feedback to CPG circuits. Extrapolating from previous studies this suggests that CPG output may be adversely affected, particularly during the transition from stance to swing phase and regarding the development of clonus. Studies also indicate that load receptors contribute to leg extensor activity during standing and walking[26]. Reduced loading may affect this mechanism.

Rollator walking reduces hip extension range of movement [70] which may inhibit normal transition from stance to swing phase.[51] 


\section{Changes in muscle afferent input change CPG output}

As muscle afferents also affect CPG output, $[53,54]$ reduced muscle activity due to cane[68] and rollator use[71] and changes in muscle length due to rollator use[70] may impact CPG activity. A reduction in plantar flexion activity is needed to initiate swing phase.[53] The effect of consistently reduced muscle activity on this transition has to our knowledge not been investigated. Unloading the ankle plantar flexors during the stance phase of walking reduced soleus activity by $50 \%$ in early and mid-stance.[54] As these muscles are unloaded during cane and rollator use, soleus activity may be reduced in a similar way. Although the exact effect of altered muscle afferent input due to use of these devices on CPGs remains unclear, extrapolation from other studies suggests that the altered input will change output.

It has been demonstrated that SIN activity changes depending on postural sets and motor goals. [27] The exact mechanisms which influence these changes remain unclear but it is likely, as with other aspects of SIN and CPG activity, that peripheral inputs play an important role. Altered multisensory afferent input could change IN inhibitory and excitatory activity.

When patients walk with assistive devices that require hands, the upper limbs must be decoupled from four limb CPG control which requires increased cortical activity.[32, 48] This may have detrimental effects on patients with reduced balance, who often use more cognitive resources to maintain stability.[72]

\section{Alterations in afferent information when using a cane which may influence motor output.}

\section{Arms decoupled from four-limb CPG control requiring increased cognitive resources \\ 2. Hip joint loading reduced on opposite side to cane use (hemiplegic hip)}

3. N.A.

4. Muscle activity reduced on opposite side to cane use (hemiplegic muscles)

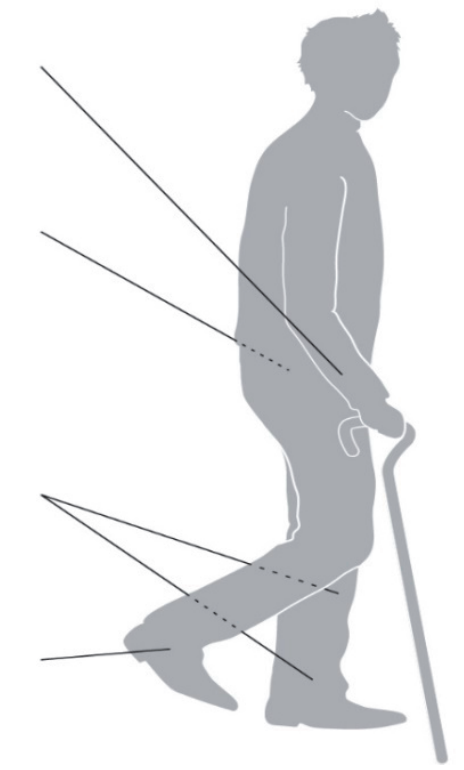

5. Collisions between cane and foot may give confusing cutaneous inputs

Figure 7. Changes in afferent input due to cane use which may influence CPG output 
Alterations in afferent information when a rollator which may influence motor output

\begin{abstract}
1. Arms decoupled from four-limb CPG control requiring increased cognitive resources.
\end{abstract}

2. Increased loading at the arms reduces loading at the hips

3. Hip extension range of motion reduced, potentially influencing transition to swing phase.

4. Muscle activation levels reduced.

5. Collisions between wheels and feet may give confusing cutaneous inputs

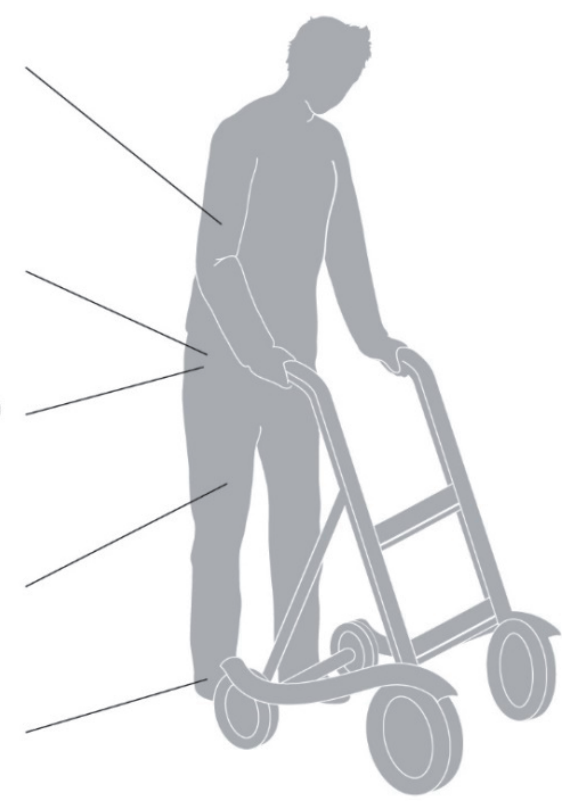

Figure 8. Changes in afferent input due to rollator use which may influence CPG output

\title{
E. Plasticity of spinal circuitry
}

Structural and functional changes to the cerebral cortex following stroke, and the importance of neural plasticity for motor recovery have been well documented.[73]

Recent studies also indicate that changes in spinal cord circuitry post-stroke contribute to functional recovery.[15] Animal studies have shown that improved function is associated with increased axonal sprouting from the uninjured corticospinal tract "with numerous axons observed crossing the midline in the brainstem and spinal cord and terminating in denervated grey matter". Significantly, factors which influenced these spinal plastic changes positively influenced sensorimotor recovery. [74] Although in this study the manipulating factors were chemical in nature (ChABC), the principal that elements which influence spinal plasticity can positively impact motor recovery post-stroke may be significant for physical rehabilitation. The sensory afferents discussed in this review, which have been shown to influence SIN and CPG in- and output, may provide a route to influence spinal plasticity and improve post-stroke function. It has been shown in spinal injury patients that afferent influence on spinal networks can play an important role in functional reorganisation of motor output.[50] and that spinal and cortical plasticity is influenced by the pattern of sensory afferent feedback.[75] 


\section{CONCLUSIONS}

These aspects of neural control and recovery should be considered during the prescription of walking-aids. It may be that assistive walking devices should allow normal joint loading, have minimal effect on muscle activity and length during walking and not require the use of hands. Some orthoses are known to fulfil these criteria. [68] These factors may enable afferent input typical of healthy gait. Normal sensory input may facilitate optimal CPG activity and therefore motor output. This may positively impact spinal plasticity and functional recovery. As walking aids are often used for long periods during the day, meaning high repetition, the influence on plasticity may be significant. Clinical research is needed to investigate these hypotheses.

Conflict of Interest Statement The authors declare that there is no conflict of interest.

Acknowledgements. The authors would like to thank Greet Mommen, MSc. (scientific illustrator) for her invaluable contribution to the figures and medical illustrations in this review. 


\section{REFERENCES}

[1] Taub E. The behavior-analytic origins of constraint-induced movement therapy: an example of behavioral neurorehabilitation. Behav Anal. 2012 Fall;35(2):155-78.

[2] JM V, EEH vW, RPS vP, HIM H, MB R, PhJ vdW, et al. Royal Dutch Society for Physical Therapy - Clinical Practice Guideline for Physical Therapy in patients with stroke. 2014.

[3] States RA, Pappas E, Salem Y. Overground physical therapy gait training for chronic stroke patients with mobility deficits. Cochrane Database Syst Rev. 2009(3):Cd006075.

[4] Lennon S. Physiotherapy practice in stroke rehabilitation: a survey. Disabil Rehabil. 2003 May 6;25(9):455-61.

[5] Kollen BJ, Lennon S, Lyons B, Wheatley-Smith L, Scheper M, Buurke JH, et al. The effectiveness of the Bobath concept in stroke rehabilitation: what is the evidence? Stroke. 2009 Apr;40(4):e89-97.

[6] Jeong YG, Jeong YJ, Myong JP, Koo JW. Which type of cane is the most efficient, based on oxygen consumption and balance capacity, in chronic stroke patients? Gait Posture. 2015 Feb;41(2):493-8.

[7] Polese JC, Teixeira-Salmela LF, Nascimento LR, Faria CD, Kirkwood RN, Laurentino GC, et al. The effects of walking sticks on gait kinematics and kinetics with chronic stroke survivors. Clin Biomech (Bristol, Avon). 2012 Feb;27(2):131-7.

[8] Getting PA. Emerging principles governing the operation of neural networks. Annu Rev Neurosci. 1989;12:185-204.

[9] Squire LR, Berg D, Bloom FE, Lac Sd, Ghosh A, Spitzer NC. Fundamental Neuroscience. 4th ed: Elsevier Inc 2013.

[10] Jankowska E, Hammar I. Spinal interneurones; how can studies in animals contribute to the understanding of spinal interneuronal systems in man? Brain Res Brain Res Rev. 2002 Oct;40(1-3):19-28.

[11] Capaday C, Ethier C, Van Vreeswijk C, Darling WG. On the functional organization and operational principles of the motor cortex. Front Neural Circuits. 2013;7:66.

[12] Harel R, Asher I, Cohen O, Israel Z, Shalit U, Yanai Y, et al. Computation in spinal circuitry: lessons from behaving primates. Behav Brain Res. 2008 Dec 12;194(2):119-28.

[13] Wirz M, Colombo G, Dietz V. Long term effects of locomotor training in spinal humans. J Neurol Neurosurg Psychiatry. 2001 Jul;71(1):93-6.

[14] Arya KN, Pandian S. Interlimb neural coupling: implications for poststroke hemiparesis. Ann Phys Rehabil Med. 2014 Dec;57(9-10):696-713.

[15] Sist B, Fouad K, Winship IR. Plasticity beyond peri-infarct cortex: spinal up regulation of structural plasticity, neurotrophins, and inflammatory cytokines during recovery from cortical stroke. Exp Neurol. 2014 Feb;252:47-56.

[16] Edgley SA. Organisation of inputs to spinal interneurone populations. J Physiol. 2001 May 15;533(Pt 1):51-6.

[17] Jankowska E. Spinal interneuronal networks in the cat: elementary components. Brain Res Rev. 2008 Jan;57(1):46-55.

[18] Kandel ER, Schwartz JH, Jessell TM, Siegelbaum SA, Hudspeth AJ. Principles of Neural Science2013.

[19] Shinoda Y, Yokota J, Futami T. Divergent projection of individual corticospinal axons to motoneurons of multiple muscles in the monkey. Neurosci Lett. 1981 Apr 9;23(1):7-12.

[20] Jankowska E, Edgley S. Interactions between pathways controlling posture and gait at the level of spinal interneurones in the cat. Prog Brain Res. 1993;97:161-71.

[21] Kiehn O, Dougherty KJ, Hagglund M, Borgius L, Talpalar A, Restrepo CE. Probing spinal circuits controlling walking in mammals. Biochem Biophys Res Commun. 2010 May 21;396(1):11-8.

[22] Windhorst U. Muscle proprioceptive feedback and spinal networks. Brain Res Bull. 2007 Jul 12;73(46):155-202.

[23] Kiehn O. Locomotor circuits in the mammalian spinal cord. Annu Rev Neurosci. 2006;29:279-306.

[24] Purves D, George J A, Fitzpatrick D, William C H, Anthony-Samuel L, Leonard E W. Neuroscience. Fifth ed. MA USA: Sinauer Associates, Inc 2012. 
[25] Conway BA, Hultborn $\mathrm{H}$, Kiehn O. Proprioceptive input resets central locomotor rhythm in the spinal cat. Exp Brain Res. 1987;68(3):643-56.

[26] Dietz V, Gollhofer A, Kleiber M, Trippel M. Regulation of bipedal stance: dependency on "load" receptors. Exp Brain Res. 1992;89(1):229-31.

[27] Jankowska E. Interneuronal relay in spinal pathways from proprioceptors. Prog Neurobiol. 1992;38(4):335-78.

[28] Nishimaru H, Restrepo CE, Kiehn O. Activity of Renshaw cells during locomotor-like rhythmic activity in the isolated spinal cord of neonatal mice. J Neurosci. 2006 May 17;26(20):5320-8.

[29] Alvarez FJ, Fyffe RE. The continuing case for the Renshaw cell. J Physiol. 2007 Oct 1;584(Pt 1):31-45.

[30] Takakusaki K. Neurophysiology of gait: from the spinal cord to the frontal lobe. Mov Disord. 2013 Sep;28(11):1483-91.

[31] Zhang Y, Narayan S, Geiman E, Lanuza GM, Velasquez T, Shanks B, et al. V3 spinal neurons establish a robust and balanced locomotor rhythm during walking. Neuron. 2008 Oct 9;60(1):84-96.

[32] Dietz V. Spinal cord pattern generators for locomotion. Clin Neurophysiol. 2003 Aug;114(8):1379-89.

[33] Sandrini G, Serrao M, Rossi P, Romaniello A, Cruccu G, Willer JC. The lower limb flexion reflex in humans. Prog Neurobiol. 2005 Dec;77(6):353-95.

[34] Fetz EE, Perlmutter SI, Prut Y, Seki K, Votaw S. Roles of primate spinal interneurons in preparation and execution of voluntary hand movement. Brain Res Brain Res Rev. Netherlands2002:53-65.

[35] Dietz V, Müller R, Colombo G. Locomotor activity in spinal man: significance of afferent input from joint and load receptors. Brain. 2002 Dec;125(Pt 12):2626-34.

[36] Nielsen JB. How we walk: central control of muscle activity during human walking. Neuroscientist. 2003 Jun;9(3):195-204.

[37] Dietz V. Neuronal plasticity after a human spinal cord injury: positive and negative effects. Exp Neurol. 2012 May;235(1):110-5.

[38] Grillner S. Neuroscience. Human locomotor circuits conform. Science. 2011 Nov;334(6058):912-3.

[39] Squire LR, Berg D, Bloom FE, Lac Sd, Ghosh A, Spitzer NC. Fundamental Neuroscience. 3rd ed: Elsevier Inc 2008.

[40] Molinari M. Plasticity properties of CPG circuits in humans: impact on gait recovery. Brain Res Bull. 2009 Jan;78(1):22-5.

[41] Cheron G, Duvinage M, De Saedeleer C, Castermans T, Bengoetxea A, Petieau M, et al. From spinal central pattern generators to cortical network: integrated $\mathrm{BCl}$ for walking rehabilitation. Neural Plast. 2012;2012:375148.

[42] van Hedel HJ, Dietz V. Rehabilitation of locomotion after spinal cord injury. Restor Neurol Neurosci. 2010;28(1):123-34.

[43] Grillner S. Neurobiological bases of rhythmic motor acts in vertebrates. Science. 1985 Apr 12;228(4696):143-9.

[44] Cappellini G, Ivanenko YP, Poppele RE, Lacquaniti F. Motor patterns in human walking and running. J Neurophysiol. 2006 Jun;95(6):3426-37.

[45] Danner SM, Hofstoetter US, Freundl B, Binder H, Mayr W, Rattay F, et al. Human spinal locomotor control is based on flexibly organized burst generators. Brain. 2015 Jan 12.

[46] Delwaide PJ, Crenna P. Cutaneous nerve stimulation and motoneuronal excitability. II: Evidence for nonsegmental influences. J Neurol Neurosurg Psychiatry. 1984 Feb;47(2):190-6.

[47] Dietz V. Spinal cord lesion: effects of and perspectives for treatment. Neural Plast. 2001;8(1-2):83-90.

[48] Dietz V. Do human bipeds use quadrupedal coordination? Trends Neurosci. 2002 Sep;25(9):462-7.

[49] Debaere F, Swinnen SP, Beatse E, Sunaert S, Van Hecke P, Duysens J. Brain areas involved in interlimb coordination: a distributed network. Neuroimage. 2001 Nov;14(5):947-58.

[50] Harkema SJ. Plasticity of interneuronal networks of the functionally isolated human spinal cord. Brain Res Rev. 2008 Jan;57(1):255-64.

[51] Pearson KG. Generating the walking gait: role of sensory feedback. Prog Brain Res. 2004;143:123-9.

[52] Petersen TH, Willerslev-Olsen M, Conway BA, Nielsen JB. The motor cortex drives the muscles during walking in human subjects. J Physiol. 2012 May 1;590(Pt 10):2443-52. 


\section{Chapter 2}

[53] Pearson KG, Misiaszek JE, Fouad K. Enhancement and resetting of locomotor activity by muscle afferents. Ann N Y Acad Sci. 1998 Nov;860:203-15.

[54] Sinkjaer T, Andersen JB, Ladouceur M, Christensen LO, Nielsen JB. Major role for sensory feedback in soleus EMG activity in the stance phase of walking in man. J Physiol. 2000 Mar 15;523 Pt 3:817-27.

[55] McCrea DA. Spinal circuitry of sensorimotor control of locomotion. J Physiol. 2001 May 15;533(Pt 1):4150.

[56] Pearson KG. Spinal cord injury reveals unexpected function of cutaneous receptors. J Neurophysiol. 2003 Dec;90(6):3583-4.

[57] Rossignol S, Dubuc R, Gossard JP. Dynamic sensorimotor interactions in locomotion. Physiol Rev. 2006 Jan;86(1):89-154.

[58] Drew T, Prentice S, Schepens B. Cortical and brainstem control of locomotion. Prog Brain Res. 2004;143:251-61.

[59] Dietz V, Horstmann GA, Berger W. Interlimb coordination of leg-muscle activation during perturbation of stance in humans. J Neurophysiol. 1989 Sep;62(3):680-93.

[60] Kearney RE, Lortie M, Stein RB. Modulation of stretch reflexes during imposed walking movements of the human ankle. J Neurophysiol. 1999 Jun;81(6):2893-902.

[61] Sinkjaer T, Andersen JB, Larsen B. Soleus stretch reflex modulation during gait in humans. J Neurophysiol. 1996 Aug;76(2):1112-20.

[62] Mynark RG, Koceja DM. Comparison of soleus H-reflex gain from prone to standing in dancers and controls. Electroencephalogr Clin Neurophysiol. 1997 Apr;105(2):135-40.

[63] Voss M, Ingram JN, Haggard P, Wolpert DM. Sensorimotor attenuation by central motor command signals in the absence of movement. Nat Neurosci. 2006 Jan;9(1):26-7.

[64] Haggard P, Whitford B. Supplementary motor area provides an efferent signal for sensory suppression. Brain Res Cogn Brain Res. 2004 Mar;19(1):52-8.

[65] Dietz V. Body weight supported gait training: from laboratory to clinical setting. Brain Res Bull. 2009 Jan $15 ; 78(1):$ I-vi.

[66] Recktenwald MR, Hodgson JA, Roy RR, Riazanski S, McCall GE, Kozlovskaya I, et al. Effects of spaceflight on rhesus quadrupedal locomotion after return to 1G. J Neurophysiol. 1999 May;81(5):2451-63.

[67] Ajemian S, Thon D, Clare P, Kaul L, Zernicke RF, Loitz-Ramage B. Cane-assisted gait biomechanics and electromyography after total hip arthroplasty. Arch Phys Med Rehabil. 2004 Dec;85(12):1966-71.

[68] Maguire C, Sieben JM, Frank M, Romkes J. Hip abductor control in walking following stroke -- the immediate effect of canes, taping and TheraTogs on gait. Clin Rehabil. 2010 Jan;24(1):37-45.

[69] Tung JY, Gage WH, Poupart P, Mcllroy WE. Upper limb contributions to frontal plane balance control in rollator-assisted walking. Assist Technol. 2014 Spring;26(1):15-21; quiz 2-3.

[70] Alkjaer T, Larsen PK, Pedersen G, Nielsen LH, Simonsen EB. Biomechanical analysis of rollator walking. Biomed Eng Online. 2006;5:2.

[71] Zorica S, Jacqueline R, Amir T, Clare M. Walking with a four wheeled walker (rollator) significantly reduces EMG lower limb muscle activity in healthy subjects. Journal of Bodywork and Movement Therapies. 2015.

[72] Lamoth CJ, van Deudekom FJ, van Campen JP, Appels BA, de Vries OJ, Pijnappels M. Gait stability and variability measures show effects of impaired cognition and dual tasking in frail people. J Neuroeng Rehabil. 2011;8:2.

[73] Nudo RJ. Postinfarct cortical plasticity and behavioral recovery. Stroke. 2007 Feb;38(2 Suppl):840-5.

[74] Starkey ML, Bartus K, Barritt AW, Bradbury EJ. Chondroitinase ABC promotes compensatory sprouting of the intact corticospinal tract and recovery of forelimb function following unilateral pyramidotomy in adult mice. Eur J Neurosci. 2012 Dec;36(12):3665-78.

[75] Knikou M. Neural control of locomotion and training-induced plasticity after spinal and cerebral lesions. Clin Neurophysiol. 2010 Oct;121(10):1655-68. 


\section{Chapter}

Movement goals stored in the cortex and muscle synergies to reduce redundancy - an efficient system to control movement and gait. How does it work, what changes following stroke and what are the consequences for rehabilitation and the use of walking-aids? A literature review. 


\section{ABSTRACT}

Current knowledge of neural and neuromuscular processes controlling gait and movement as well as an understanding of how these mechanisms change following stroke is an important basis for the development of effective rehabilitation interventions. To support the translation of findings from basic research into useful treatments in clinical practice, up-to-date neuroscience should be presented in forms accessible to all members of the multidisciplinary team. In this review we discuss aspects of cortical control of gait and movement, muscle synergies as a way of translating cortical commands into specific muscle activity and as an efficient means of reducing neural and musculoskeletal redundancy. We discuss how these mechanisms change following stroke, potential consequences for gait rehabilitation and the use of walking-aids as well as areas requiring further research.

Keywords: cerebral cortex, neuronal networks, muscle synergies, cerebrovascular stroke, gait rehabilitation 


\section{INTRODUCTION}

An up-to-date knowledge of neural and neuromuscular processes controlling movement as well as an understanding of how these mechanisms change following stroke, is an important basis for developing effective rehabilitation interventions. In this article we discuss research findings regarding a)cortical involvement in movement and gait control and b) "muscle-synergies" which according to a large body of evidence are the means by which cortical commands are converted into muscle actions. c)We discuss how these mechanisms change post stroke and possible consequences for rehabilitation and the use of walking-aids.

\section{A) MOTOR CORTEX}

\section{Movement maps}

Literature describes the encoding of complex movements or components of movement sequences by neuronal networks (alternatively called motor-maps) in the cerebral cortex.[1-3] Motor networks are constructed and refined to produce actions.[1, 4]This analysis of cortical representation is relatively new and challenges long standing ideas regarding the existence of clearly defined motor and sensory homunculi.

Motor-maps have been investigated by directly stimulating the cerebral cortex. In early studies, surface stimulation, which provided crude localization, was applied to the motor cortices of animals for brief periods, typically 20ms, evoking muscle twitches in different parts of the body.[5] Similar studies were later carried out in human surgical patients leading to the creation of maps in which stimulated cortical points corresponded to individually activated muscles (somatotopy). These lead to the development of the motor homunculus.[6] In these early studies the motor homunculus was considered to be the primary motor-cortex(M1) which was under the command of the higher order pre-motor-cortex.[7]

By the 1960s surface stimulation of the cerebral-cortex was replaced by more precise microelectrode stimulation.[1] Although more precise, brief impulses which provoked only muscle twitches, continued to be used for several decades.[8] Only when the duration of electrical stimulation was increased, such as by Graziano et al.,[9, 10] were complex, multi-joint movements evoked. In the monkey cortex electrical stimulation was increased to $500 \mathrm{~ms}$, which is approximately the same duration required for a movement to be performed. The movements evoked represented motor tasks e.g. hand to mouth or defensive actions, rather than random complex movements. These studies were decisive in changing the understanding of the structural and functional organization of the motor-cortex. 
It is now generally agreed that a traditional view of a motor homunculus which spans the cortical surface is too simplified. $[3,4,11]$ Movements in the form of specific motor-tasks which can have multiple-representations, overlaps, and areas separated by poorly defined borders, is considered a more realistic view of topographical organization in the motor-cortex.[4]

\section{NEURONAL CIRCUITRY}

\section{Neuronal clusters}

Cortical maps have been described as "neuronal clusters that elicit complex behaviours". [12] Clusters of neurons control muscles in a particular body region, and separate clusters are interconnected into larger networks. Networks can be selectively activated and combined to produce multi-muscle, multi-jointed movements.

\section{Interconnections}

The motor-cortex is highly interconnected.[12, 13] Neuronal networks are bound together by axon collaterals that form boutons (synapses along the length of the axon) along their full length.[14] Some cortical interneurons(INs) extend across the representations for a whole limb thereby connecting territories of proximal and distal muscles.[15] Cortical neurons extend to sub-cortical structures such as the thalamus, cerebellum, basal ganglia and vestibular nuclei as well as via corticospinal pathways directly to spinal INs and motor neurone(MN) pools.

\section{Cascade of activation}

It is likely that cortical stimulation triggers a cascade of activation through horizontal and descending interconnections to distant cortical, sub-cortical and spinal inter-and motor neurons.[16] This dynamic spread of activity through neural space represents the neural activity which occurs during movement preparation, initiation and coordination. $[3,17]$ This allows long-range integrated control of multi-muscle and multi-joint movements.

\section{CORTEX TO CORTEX OR SPINAL CORD - ORGANIZATION OF CONNECTIVITY}

Axons from the motor-cortex demonstrate three types of structural organization: convergence, divergence and horizontal interconnection.[15] 


\section{Convergence}

Convergence occurs when neuronal afferents from diverse cortical regions converge on the spinal motor-neuron pool of a given muscle. This indicates that individual muscles are controlled from multiple cortical sites and can therefore be involved in multiple complex movements. For example the anterior deltoid has been shown to have multiple representations for differing functions such as postural static support during movements of the forearm and hand or movement of the whole arm to transport the hand.[14] This illustrates that muscle representation is replicated and overlapping in the motor cortex.[12]

\section{Divergence}

Divergence is demonstrated when single cortical neurons send outputs to multiple single muscle spinal neuronal pools. A single corticospinal axon may send afferents to the motor-neuron pools of multiple muscles at different levels of the spinal-cord.[15] Consequently a single cortical neuron can influence multi-muscle and multi-joint movement.

\section{Horizontal-interconnectivity}

Horizontal-interconnectivity describes the cortico-cortico networks between neuronal clusters within the motor-cortex as well as between other areas such as pre-frontal and parietal or sub-cortical structures.

Through the formation of motor movement maps each point in the cortex is connected to many muscles and each muscle is represented by many points. Therefore stimulation at discrete points in the cortex can result in activity in a distributed set of muscles.[18]

\section{MOTOR-GOALS STORED IN CORTEX}

Capaday et al. have hypothesized that the motor-cortex functions by determining which spatial, temporal and force patterns of muscle activity, or muscle-synergies, are necessary to place a limb or other body part into a particular spatial location.[13, 14] The studies by Graziano et al. which reveal a map of stored movement goals encoded as final spatial position,[9] appear to demonstrate and support this hypothesis.

The motor-goals are arranged across the cortical surface in a map based on final spatial destination of the body part. For example, premotor cortex stimulation has initiated grip movements of the hand near the mouth. Stimulation of the primary motor cortex caused hand movements to central space in front of the body resembling defensive movements.[4] When the limb was placed in different starting postures in this 
study, stimulation of the same cortical site resulted in identical end positions despite the initial differences. This suggests that a final movement goal is encoded at each cortical site rather than an unvarying sequence of muscle activation, and that different activation sequences will be selected as appropriate, to achieve the goal.

\section{Similar movements stored together}

Stimulation sites situated closely to each other induce similar movements e.g. hopping and running, although certain parameters do vary such as foot or hand position, speed or force.[2, 3] This proximity or clustering strengthens during learning.[77] There is also no apparent hierarchy in movement representation via the primary and pre-motor cortex, as was previously thought.[2]

\section{Obstacle avoidance is not pre-programmed in the cortex}

Graziano et al.[1] found that if obstacles were placed in the way of movement paths, original movement trajectories did not change. No adaptive movements occurred during stimulation. This suggests that adaptation to changing external circumstances is not preprogrammed but occurs due to online input from other areas e.g. from the parietal-cortex in response to visual stimuli or from brain-stem responses to sensory afferent feedback.

\section{b)Muscle-synergies}

\section{Achievement of movement goals through muscle-synergies}

As cortical sites encode movement goals the question arises as to how the kinetic and kinematic changes necessary to achieve the goal are brought about. A large body of research suggests that the selective recruitment and combination of embedded "muscle-synergies" offers an efficient means of providing goal directed, selective movement.[19-22]

\section{Muscle-synergy definition}

Muscle-synergies are defined as "groups of muscles with fixed ratios of activation that can be recruited by neural commands to execute a task in a feedforward or feedback manner".[23] It has been suggested that muscle-synergies represent "a library" of muscle actions that can be combined to create movements.[24] 


\section{Stored throughout the nervous system}

Muscle-synergies for different tasks appear to be encoded at different levels of the nervous system such as motor-cortex for grasping,[25] brain-stem for postural control[26] and spinal-cord for gait[27]. Many muscle-synergies are used across tasks, suggesting that they can be accessed with multiple neural mechanisms regardless of their location.[23]

\section{Central-Pattern-Generators encode muscle-synergies}

Basic locomotion is primarily under Central-Pattern-Generator(CPG) control.[28] Stimulation of specific spinal-cord-sites causes coordinated activation of muscle groups and multi-joint movements, including elements of gait. Regional organization is apparent as stimulation at different levels activates different movement elements. [29] As gait CPGs are stored in the spinal-cord, and as spinal-cord stimulation causes coordinated muscular activity, this suggests that CPGs are a form of spinal muscle-synergy.

However the importance of cortical activity even during steady unperturbed gait to support CPG activity has been emphasised in recent literature.[30] Descending cortical signals interact with spinal networks to ensure that precise changes in limb movement are appropriately integrated into the basic gait pattern. Subpopulations of motorcortical neurones are active sequentially during the step cycle particularly during swing and the initiation of swing.[27]

\section{The problem of redundancy or the gift of abundance?}

Bernstein[31] first discussed the "problem" that in order to achieve a motor-goal many different movements could be used. Each movement could be achieved with different patterns of muscle activation each innervated with different groups of neurones. Neilson et al.[32] state that several hundred functional muscles control about 110 elemental movements, confronting the nervous-system with the dilemma that an infinite number of alpha-drives patterns could produce the same movement. Bernstein termed this huge variety of possible solutions "Degrees of Freedom" (DOF), stating that for each motor task anatomical DOFs(muscles and joints), kinematic DOFs(trajectory, velocity), kinetic DOFs(force) and neural DOFs(neuronal sets) must be selected.

This in-built "redundancy" presents a problem to the central-nervous-system (CNS) if each variable must be determined. One possible solution is via muscle-synergies. This theory hypothesizes that the CNS activates groups of muscles in patterns in order to simplify movement control. This strategy allows a large variety of motor tasks to be controlled with a much smaller number of neural command signals than if each muscle were controlled individually. This is described as a low dimensional control strategy. This idea has been extensively researched in the last decade.[19, 20, 27, 33-40] Studies suggest 
that for a specific motor-goal several muscle-synergies, representing biomechanical subtasks, can be flexibly combined to produce the movement[19-22, 39, 41] Synergies may be viewed as "building blocks which simplify the construction of motor behaviours".[36] Muscle-synergy theory implies that from an endless number of possible solutions an individual develops a limited set of muscle activation patterns for motor-control.

\section{The development and refinement of muscle-synergies}

It has been claimed that "a slowly adapting synergy generator is gradually "wired-in" to the nervous system by processes that begin before birth and continue thereafter."[32] As all foetuses display similar movements, the spontaneous activity is thought to be mediated by inherited descending connectivity.[42] As these spontaneous movements are initially very variable from one repetition to the next, the connectivity is considered to be poorly focused. Nielson et al.[43,44] claim that with practice, task-specific synergies are developed and furthermore that these give rise to cortical motor maps. These ideas are supported by further studies.[36, 45] Kargo and Nitz showed in rats, that as motor skill improved, muscle-synergies became more clearly identifiable and these changes correlated with changing activity in the primary motor-cortex. The cortical activity appeared to be associated with programme (muscle-synergy) selection and tuning.

\section{Variability, fine tuning and motor learning}

Evidence indicates that muscle-synergies used to achieve specific motor-goals are generally the same within individuals across trials and between individuals for fundamental postural, balance and locomotor-goals $[21,45,46]$ as well as for newly learned skilled tasks.[36] This suggests that certain patterns of muscle activation are favoured in order to achieve specific movements. The predominance of the same patterns across individuals may reflect limitations imposed on the CNS by the physical environment and the biomechanics of the musculoskeletal system.

\section{Inter and intra-subject variability}

Although patterns of activation are "generally" consistent, variability does exist. Intrasubject variability between trials as well as inter-subject variability have been observed implying that the same movement is performed with variations in muscle coordination strategies.[36]

\section{Inter-subject variability - skill, practice and musculoskeletal constraints}

Different patterns of muscle activation can create the same resulting force vector and therefore the same movement.[36, 47, 48] Varying patterns of activation between indi- 
viduals may reflect practice or different levels of skill. Constraints imposed by the musculoskeletal system such as flexibility and strength may also influence CNS activation patterns. As the intrinsic mechanical properties between individual musculoskeletal systems vary so will the activation patterns necessary to achieve the same goal.

Frère et al.[36] found that variability across subjects, was seen primarily during complex aspects of a motor task. At this point "subject specific muscle-synergy compositions" seemed to appear. This supports the observation that some muscle-synergies are more robust than others[46] and suggests that more variability appears with increasing task difficulty.

\section{Intra-subject variability - fine tuning and motor learning}

Intra-individual trial-by-trial variations are observed when the motor-goal remains unchanged. The spatial patterns (actual muscles recruited) remain unchanged but the amplitude and timing of activation change.[42] This may reflect between trial differences in the relative contribution of each synergy although the synergies themselves are the same.[26] Ting et al. suggest that this represents a "fine tuning" by the nervous system in an attempt to balance the opposing demands of 1 . achievement of the motor goal and 2. energy efficiency. This fine tuning is based on "feedback" regarding prior performance and "feedforward" to change parameters such as muscle stiffness or trajectory to improve future performance.[47]

As skill and performance improve more consistent synergistic patterns are found.[36, 45, 46] Repeating and practicing movements could lead to the development of new muscle-synergies or change the composition and temporal activation of existing ones.[49]

Variability demonstrates that "redundancy" can be seen as "abundance" allowing creativity and improvisation.[50] It enables flexible control and the ability to adapt as conditions change, whether external or internal.[47] This facilitates the development of individual motor solutions and a motor-style.[51, 52]

\section{Muscle-synergies during healthy walking}

Four to five muscle-synergies have been identified for controlling muscle activity and coordination during human walking. $[24,41,53]$ These are implemented via the CentralPattern-Generators i.e. neural networks at spinal-cord level. These have been identified as:

Synergy 1 - Provides body support in early stance. Hip and knee extensors and hip abductors (vastus lateralis, rectus femoris, gluteus maximus, gluteus medius) are activated. 
Synergy 2 - Is the primary contributor to forward propulsion in late stance and contributes to body support. Ankle plantar flexors (gastrocnemius, soleus) are activated.

Synergy 3 - Generates dorsiflexion in early stance at heel strike and during early swing contributing to foot clearance. Tibialis anterior and rectus femoris are activated.

Synergy 4 - Functions to decelerate the leg in late swing and supports forwards propulsion of the body in early stance. Knee flexors and hip extensor (biceps femoris, semitendinosis, semimembranosis) are active during late swing and early stance.[41, 54, 55]

Some authors also describe a fifth module:

Synergy 5 -The hip flexor (iliopsoas) functions to add energy to the leg during pre-swing and swing phases and to transfer energy from the trunk to the leg during swing.[41]

These synergies specify spatial organisation of muscle activation (which muscles) and represent different biomechanical subtasks.[56] They are stored as CPGs in the spinalcord and can be recruited by different neural circuits and combined to achieve specific motor-goals.[24, 57] Changing the phase, amplitude or duration of individual musclesynergies produces the entire spectrum of locomotor behaviours from very slow walking to sprinting. [41, 54]

Study results suggest that muscle-synergies for gait are modified and used for balance reactions in standing and walking.[56].
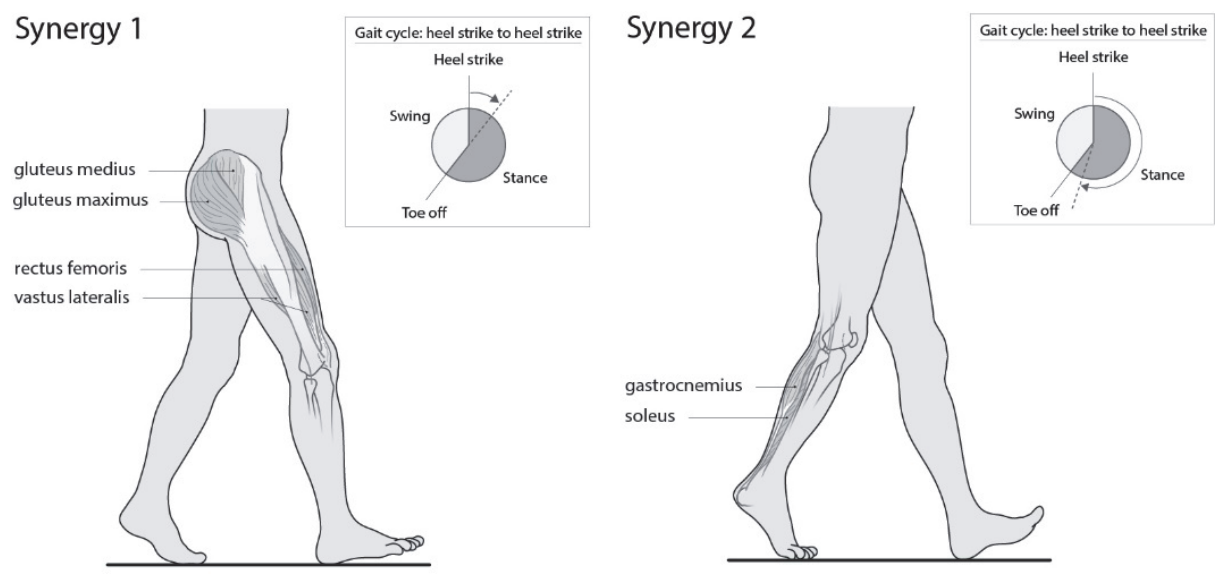

Early stance - loading response. Provides body support

Late stance - contributes to forward propulsion 


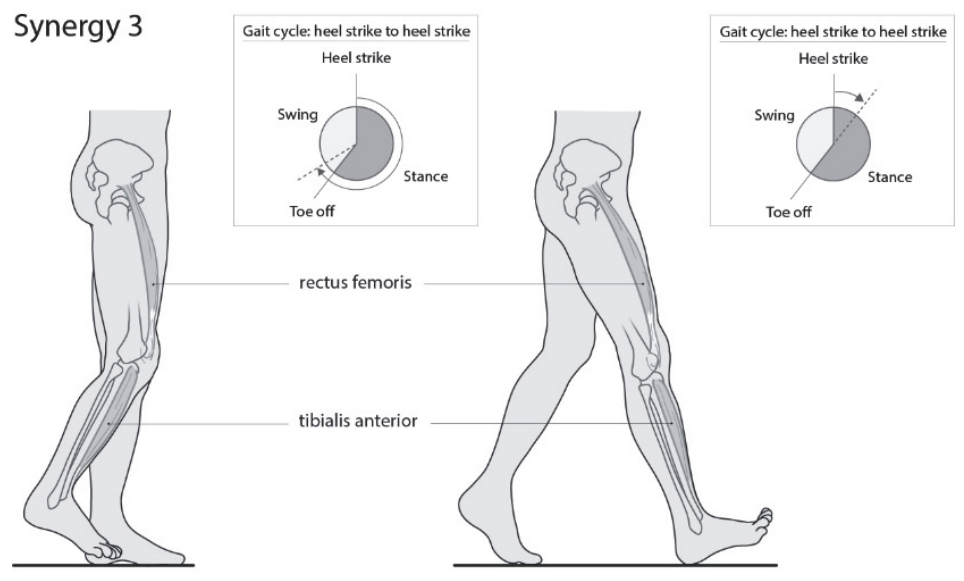

Early swing and early stance. Assists with forward swing and foot clearance

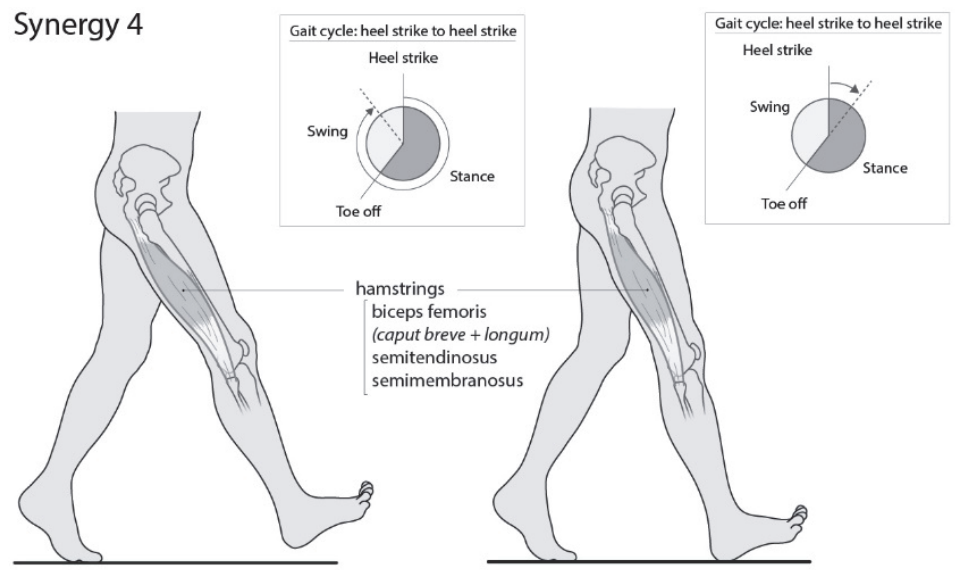

Decelerates leg in late swing

Forward propulsion in early stance

Figure1. Muscle synergies used during healthy gait

CPGs are also described as being functionally divided into parts that control i) stance and transition to swing and ii) swing phase.[58] It may be that synergies 1, 2 and 4 are associated with the stance and transition to swing function and synergies 3 and 5 with swing phase.

As studies also indicate that input from hip joint-load and movement and hip-flexor stretch receptors are important to enable the stance and transition phase of gait, it may be that these peripheral inputs are important for the appropriate recruitment of synergies 1,2 and 4. As swing-phase appears to be more influenced by central, cortical control[30], cortical input maybe more important for activation of synergies 3 and 5. 


\section{Muscle-synergies represent biomechanical subtasks}

McGowan et al.[41] demonstrated that changing body mass changed the contribution of specific muscle-synergies providing body-weight support e.g. extension in stance phase. The authors concluded that "individual modules (synergies) are associated with specific biomechanical subtasks". Further studies indicate that specific synergies are responsible for biomechanical subtasks such as force generation[20] or Centre-of-Mass kinematic variables following balance perturbations.[22]

\section{c). Stroke, cortical damage and muscle-synergies}

"Stroke patients exhibit differences in the number of muscle-synergies recruited which may reflect disruption in descending neural pathways and are correlated to deficits in motor function" $[49,59]$

The four synergies consistently described by all authors during walking in healthy subjects, are combined in the paretic leg of post-stroke subjects resulting in "new" synergies or stroke-synergies of which there may be two, three or four.[59] The recruitment of fewer stroke-synergies correlates with increased co-contraction and impairment. Fortyfive percent of subjects tested demonstrated two stroke-synergies by combining those seen in healthy subjects. One combination consisted of synergies 1,2 and 4 and was primarily active in stance phase leading to non-selective activation of extensor musculature. The second was similar to synergy 3 in healthy subjects and was primarily active in swing phase.

Thirtysix percent of subjects activated three stroke-synergies. These subjects could be further divided into two groups. Approximately half demonstrated a combination of 1 and 2 from healthy subjects. In this group the ankle plantar flexors and proximal extensor muscles remained active throughout stance phase. The differentiated recruitment of this activity by healthy subjects at the beginning and end of stance phase, was combined and unvarying throughout stance phase. The remaining synergies corresponded to synergies 3 and 4 in healthy subjects. The second group appeared to combine synergies 1 and 4 of healthy controls leading to co-activation of proximal extensors and hamstring muscles. Synergies 2 and 3 were the same as in healthy controls.

The remaining subjects with stroke demonstrated the 4 synergies characteristic of healthy controls with similar patterns and timing of muscle activation.[59] These results suggest that muscle-synergies involved in walking remain intact post-stroke but the ability to recruit them independently is diminished. 


\section{Post-stroke synergy A}

$45 \%$ of subjects tested combined

- Pre-stroke synergies 1, 2 and 4 to post-stroke synergy A

leading to non-selective activation of extensor musculature

- Recruited with pre-stroke synergy 3

Post-stroke synergy A

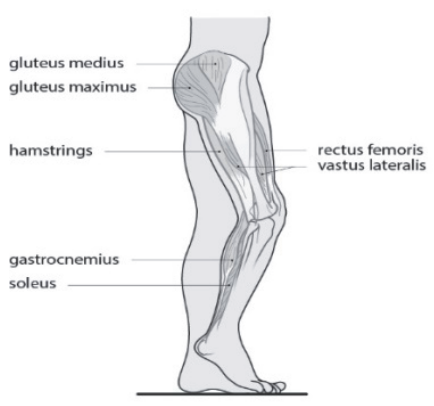

Pre-stroke synergy 3

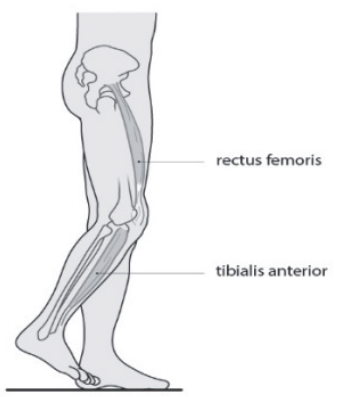

Post-stroke synergies B and C

$36 \%$ recruited 3 post-stroke synergies

Half of those who recruited 3 post-stroke synergies recruited pre-stroke synergies

1 and 2 together (post-stroke synergy B) and pre-stroke synergies 3 and 4)

Post-stroke synergy B

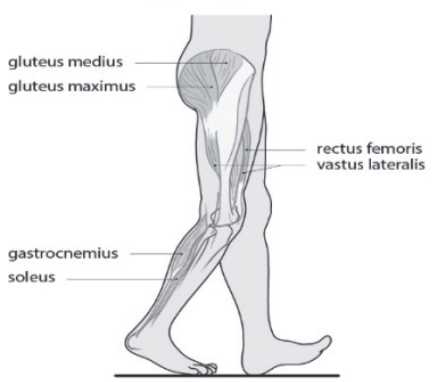

Pre-stroke synergy 3

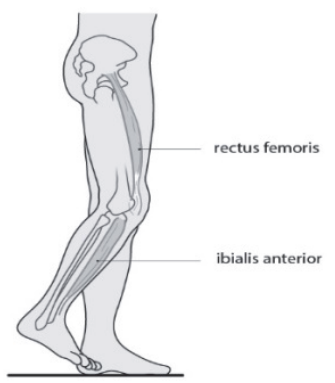

Pre-stroke synergy 4

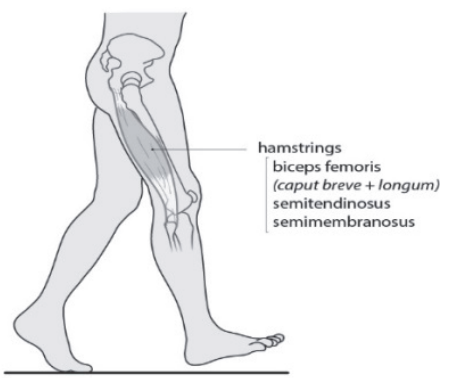

Half of those who recruited 3 post-stroke synergies recruited pre-stroke synergies 1 and 4 together (post-stroke synergy C) and pre-stroke synergies 2 and 3

Post-stroke synergy C

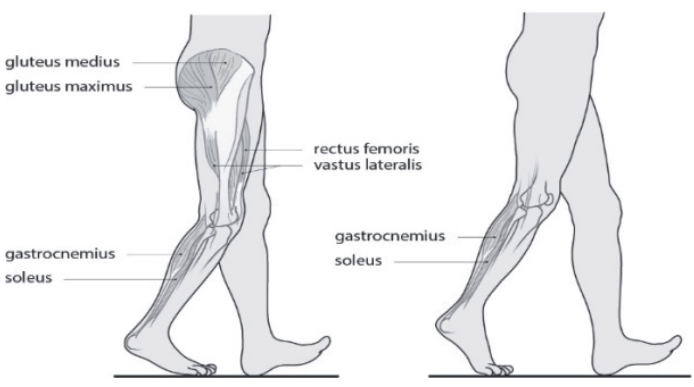

Pre-stroke synergy 3

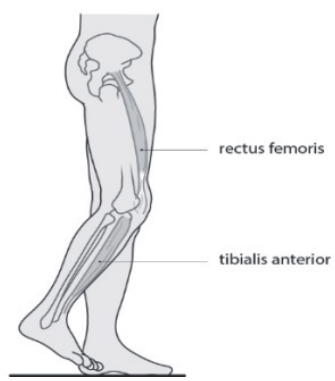

Figure 2. Common combinations of muscle synergies post-stroke 
"Damage to the cerebrum following stroke has been shown to impair the independent recruitment of muscle-synergies but not their structure in either walking or reaching." [59] This suggests that muscle-synergies are not encoded in the cortex or by cortical projections to motor neurones. However cortical input does appear to influence muscle-synergy recruitment.[39]

Anticipatory activity e.g. to avoid obstacles, has been shown to involve musclesynergy activity in atypical phases of gait. This suggests input from descending pathways can modify the timing of muscle recruitment within CPG activity.[24] The timing but not the spatial structure of muscle-synergies appears to be influenced by cortical input during walking. Consequently post-stroke damage to the cortex changes temporal but not spatial aspects of muscle-synergy recruitment. Synergies which are recruited at different times and therefore separately in healthy gait are often recruited together.[59] This would account for typical patterns of co-contraction seen in stroke patients and loss of selective motor control.

Some studies have proposed that loss of cortical input leads to increased influence of brainstem pathways on spinal networks. It is suggested that increased activity in reticulospinal and bulbospinal tracts which show more diffuse connectivity between motor neuron (MN) pools, is responsible for the merging of synergies.[59] This leads to altered MN activity and consequently altered agonistic-antagonistic strength relationships and abnormal coupling of torque generation. $[60,61]$

\section{Diminished ability to respond to changing internal and external circumstances}

When a motor goal remains constant during changing external circumstances, such as continuing to walk when encountering obstacles, a flexible recruitment of musclesynergies is required. Cortical inputs are decisive in this process.[24] Equally, changes in the internal environment such as within musculoskeletal structures, demand adaptability in recruitment of muscle-synergies to achieve the same motor task.[41] Many musculoskeletal changes occur as primary or secondary effects of stroke potentially altering the components of biomechanical sub-tasks of a movement. For example increases or decreases in muscle tone may change resistance to movement requiring altered force generation, changes in muscle length may alter lever length necessitating kinematic adaptations.

\section{Consequences for post-stroke rehabilitation and the use of walking-aids.}

As the ability to respond to internal and external environmental changes via selective recruitment of muscle-synergies is diminished post stroke, the difficulty of achieving motor-goals is increased. Internal structural changes could be seen as the equivalent of placing an obstacle in the path when walking. This indicates on a neural level, that sec- 
ondary musculoskeletal changes, such as loss of strength and flexibility, should be minimised in rehabilitation post stroke. It suggests that the preparation of musculoskeletal structures e.g. via stretching, sensory input or specific muscle activation before functional movement training may optimise the ability to use pre-stroke muscle synergies to complete a motor task and reduce the need for adaptation.

\section{Neural changes post-stroke}

As stroke primarily disrupts cortical or subcortical networks, the muscle-synergies which control locomotion, encoded as spinal CPGs, initially remain intact post-stroke. Due to changes in descending input, the ability to selectively recruit the synergies is diminished leading to the simultaneous activation of several synergies when walking.

Studies indicate that neuroplastic changes in spinal-cord circuitry occur in poststroke patients $[62,63]$ which are use dependent.[64, 65] Thus, repeated activation of several muscle-synergies together, may lead to the structural merging of neural circuitry at spinal level into one synergy. This idea is supported by studies which suggest that repeating movements change the composition and temporal activation of synergies.[49] Such secondary changes could reinforce poor selective control and the corresponding reduction in function.

\section{Gait Rehabilitation}

Task specific, high dosage practice of functional activities positively influences cortical and spinal plasticity $[66,67]$ and improves functional outcomes. $[68,69]$ Taken together, these findings imply that rehabilitation interventions which facilitate the selective activation of separate synergies during walking, with high repetitions may be beneficial. Such interventions used early after stroke may allow the structure of pre-stroke CPGs to be maintained and prevent merging. This in turn may improve functional outcomes. Secondly, as changes in muscle-synergy definition appear to influence cortical representation of these movements[36, 45], specific practice may positively influence the recovery of cortical neuronal networks controlling gait. Such interventions used in chronic stroke patients may allow the re-emergence of pre-stroke synergies.

\section{Treadmill and robotic training}

These neural processes may partially explain the unclear mechanisms behind some evidence based gait interventions, such as body-weight supported treadmill training and robotic-assisted gait training in stroke patients.[70] One consistently important aspect of these interventions is the maintenance of a kinematic pattern based on healthy gait.[71, 72] Locomotor devices guide the hip and knee joints in sagittal-plane trajectories approximating symmetrical, reciprocal locomotion.[72] Typical flexion and 
extension movement ranges at the hip, knee, and ankle joints as well as symmetrical and equal step length, weight shift and weight-bearing onto the hemiplegic leg during stance phase are facilitated during these interventions.[71] Mass patterns of movement, typical of merged muscle-synergies, are therefore prevented. Pre-stroke gait patterns are facilitated both passively and actively via appropriate sensory input. Therapist provided peripheral input combined with treadmill training appears to be the most effective in increasing functional outcomes.[72] Thus peripheral feedback into spinalcircuitry from normally aligned and promptly activated joint, muscle and cutaneous sensory afferents, may mirror peripheral afferent feedback from healthy gait, supporting the activation or re-formation of spinal circuits resembling pre-stroke CPGs and muscle-synergies. Increased selective control of synergies correlates with improved function.[59]

\section{Walking-aids}

Walking-aids, often used for long periods throughout the day, equally enable high dosage, repetitive, task-oriented movement, and influence sensory input into spinalcircuitry. To encourage the selective activation of muscle-synergies during walking, peripheral sensory feedback due to walking aid use should also mirror that of healthy gait as far as possible. Canes and rollators which reduce joint-loading[73, 74] and change muscle activity and joint angles during walking[73, 75] may not be optimal. From a neuroscientific perspective, walking-aids which enable independent walking with minimal alterations to kinetic and kinematic variables may be more effective. However further clinical research is required to evaluate whether and how gait interventions and walking-aids influence the structure and selective recruitment of musclesynergies and whether this impacts function. Such knowledge may assist in the development of evidence based guidelines for the prescription of walking aids post-stroke.

Conflict of Interest Statement. The authors declare that there is no conflict of interest. Acknowledgements. The authors would like to thank Greet Mommen, MSc. (scientific illustrator) for her invaluable contribution to the figures and medical illustrations in this review. 


\section{REFERENCES}

[1] Graziano M. The organization of behavioral repertoire in motor cortex. Annu Rev Neurosci. 2006;29:105-34.

[2] Graziano MS, Aflalo TN, Cooke DF. Arm movements evoked by electrical stimulation in the motor cortex of monkeys. J Neurophysiol. 2005 Dec;94(6):4209-23.

[3] Harrison TC, Murphy TH. Motor maps and the cortical control of movement. Curr Opin Neurobiol. 2014 Feb;24(1):88-94.

[4] Graziano MS, Aflalo TN. Mapping behavioral repertoire onto the cortex. Neuron. United States2007:239-51.

[5] Taylor CS, Gross CG. Twitches versus movements: a story of motor cortex. Neuroscientist. 2003 Oct;9(5):332-42.

[6] Schott GD. Penfield's homunculus: a note on cerebral cartography. J Neurol Neurosurg Psychiatry. 1993 Apr;56(4):329-33.

[7] Fulton J. Physiology of the Nervous System. New York: Oxford University Press 1938.

[8] Graziano MS, Taylor CS, Moore T, Cooke DF. The cortical control of movement revisited. Neuron. United States2002:349-62.

[9] Graziano MS, Taylor CS, Moore T. Complex movements evoked by microstimulation of precentral cortex. Neuron. United States2002:841-51.

[10] Cooke DF, Taylor CS, Moore T, Graziano MS. Complex movements evoked by microstimulation of the ventral intraparietal area. Proc Natl Acad Sci U S A. United States2003:6163-8.

[11] Rizzolatti G, Luppino G. The cortical motor system. Neuron. United States2001:889-901.

[12] Levine AJ, Lewallen KA, Pfaff SL. Spatial organization of cortical and spinal neurons controlling motor behavior. Curr Opin Neurobiol. 2012 Oct;22(5):812-21.

[13] Capaday C. The integrated nature of motor cortical function. Neuroscientist. 2004 Jun;10(3):207-20.

[14] Capaday C, Ethier C, Van Vreeswijk C, Darling WG. On the functional organization and operational principles of the motor cortex. Front Neural Circuits. 2013;7:66.

[15] Squire LR, Berg D, Bloom FE, Lac Sd, Ghosh A, Spitzer NC. Fundamental Neuroscience. 4th ed: Elsevier Inc 2013.

[16] Stroh A, Adelsberger H, Groh A, Ruhlmann C, Fischer S, Schierloh A, et al. Making waves: initiation and propagation of corticothalamic Ca2+ waves in vivo. Neuron. 2013 Mar 20;77(6):1136-50.

[17] Churchland MM, Cunningham JP, Kaufman MT, Foster JD, Nuyujukian P, Ryu SI, et al. Neural population dynamics during reaching. Nature. England2012:51-6.

[18] Sanes JN, Schieber MH. Orderly somatotopy in primary motor cortex: does it exist? Neuroimage. United States2001:968-74.

[19] Chvatal SA, Torres-Oviedo G, Safavynia SA, Ting LH. Common muscle synergies for control of center of mass and force in nonstepping and stepping postural behaviors. J Neurophysiol. United States2011:999-1015.

[20] Ting LH, Macpherson JM. A limited set of muscle synergies for force control during a postural task. J Neurophysiol. United States2005:609-13.

[21] Torres-Oviedo G, Macpherson JM, Ting LH. Muscle synergy organization is robust across a variety of postural perturbations. J Neurophysiol. United States2006:1530-46.

[22] Safavynia SA, Ting LH. Sensorimotor feedback based on task-relevant error robustly predicts temporal recruitment and multidirectional tuning of muscle synergies. J Neurophysiol. United States2013:31-45.

[23] Safavynia SA, Ting LH. Task-level feedback can explain temporal recruitment of spatially fixed muscle synergies throughout postural perturbations. J Neurophysiol. United States2012:159-77.

[24] Chvatal SA, Ting LH. Voluntary and reactive recruitment of locomotor muscle synergies during perturbed walking. J Neurosci. 2012 Aug 29;32(35):12237-50.

[25] Overduin SA, d'Avella A, Roh J, Bizzi E. Modulation of muscle synergy recruitment in primate grasping. J Neurosci. United States2008:880-92.

[26] Torres-Oviedo G, Ting LH. Muscle synergies characterizing human postural responses. J Neurophysiol. 2007 Oct;98(4):2144-56.

[27] Drew T, Kalaska J, Krouchev N. Muscle synergies during locomotion in the cat: a model for motor cortex control. J Physiol. England2008:1239-45. 


\section{Chapter 3}

[28] Molinari M. Plasticity properties of CPG circuits in humans: impact on gait recovery. Brain Res Bull. 2009 Jan;78(1):22-5.

[29] Bizzi E, Giszter SF, Loeb E, Mussa-Ivaldi FA, Saltiel P. Modular organization of motor behavior in the frog's spinal cord. Trends Neurosci. 1995 Oct;18(10):442-6.

[30] Petersen TH, Willerslev-Olsen M, Conway BA, Nielsen JB. The motor cortex drives the muscles during walking in human subjects. J Physiol. 2012 May 1;590(Pt 10):2443-52.

[31] N B. The coordination and regulation of movements. Oxford: Pergamon Press 1967.

[32] Neilson PD, Neilson MD. Motor maps and synergies. Hum Mov Sci. 20052005 Oct-Dec;24(5-6):774-97.

[33] Overduin SA, d'Avella A, Carmena JM, Bizzi E. Microstimulation activates a handful of muscle synergies. Neuron. 2012 Dec 20;76(6):1071-7.

[34] Berniker M, Jarc A, Bizzi E, Tresch MC. Simplified and effective motor control based on muscle synergies to exploit musculoskeletal dynamics. Proc Natl Acad Sci U S A. United States2009:7601-6.

[35] Fautrelle L, Ballay Y, Bonnetblanc F. Muscular synergies during motor corrections: investigation of the latencies of muscle activities. Behav Brain Res. Netherlands: 2010 Elsevier B.V 2010:428-36.

[36] Frere J, Hug F. Between-subject variability of muscle synergies during a complex motor skill. Front Comput Neurosci. 2012;6:99.

[37] McKay JL, Ting LH. Optimization of muscle activity for task-level goals predicts complex changes in limb forces across biomechanical contexts. PLoS Comput Biol. United States2012:e1002465.

[38] Robert T, Zatsiorsky VM, Latash ML. Multi-muscle synergies in an unusual postural task: quick shear force production. Exp Brain Res. 2008 May;187(2):237-53.

[39] Torres-Oviedo G, Ting LH. Subject-specific muscle synergies in human balance control are consistent across different biomechanical contexts. J Neurophysiol. United States2010:3084-98.

[40] Weiss EJ, Flanders M. Muscular and postural synergies of the human hand. J Neurophysiol. United States2004:523-35.

[41] McGowan CP, Neptune RR, Clark DJ, Kautz SA. Modular control of human walking: Adaptations to altered mechanical demands. J Biomech. 2010 Feb;43(3):412-9.

[42] Hubli M, Dietz V. The physiological basis of neurorehabilitation--locomotor training after spinal cord injury. J Neuroeng Rehabil. 2013;10:5.

[43] Neilson PD, Neilson MD. An overview of adaptive model theory: solving the problems of redundancy, resources, and nonlinear interactions in human movement control. J Neural Eng. 2005 Sep;2(3):S279-312.

[44] Neilson PD, Neilson MD. On theory of motor synergies. Hum Mov Sci. Netherlands: 2010 Elsevier B.V 2010:655-83.

[45] Kargo WJ, Nitz DA. Early skill learning is expressed through selection and tuning of cortically represented muscle synergies. J Neurosci. United States2003:11255-69.

[46] Hug F, Turpin NA, Guével A, Dorel S. Is interindividual variability of EMG patterns in trained cyclists related to different muscle synergies? J Appl Physiol. 2010 Jun;108(6):1727-36.

[47] Ting LH, van Antwerp KW, Scrivens JE, McKay JL, Welch TD, Bingham JT, et al. Neuromechanical tuning of nonlinear postural control dynamics. Chaos. 2009 Jun;19(2):026111.

[48] Bunderson NE, McKay JL, Ting LH, Burkholder TJ. Directional constraint of endpoint force emerges from hindlimb anatomy. J Exp Biol. 2010 Jun;213(Pt 12):2131-41.

[49] Safavynia SA, Torres-Oviedo G, Ting LH. Muscle Synergies: Implications for Clinical Evaluation and Rehabilitation of Movement. Top Spinal Cord Inj Rehabil. 2011;17(1):16-24.

[50] Latash ML, Anson JG. Synergies in health and disease: relations to adaptive changes in motor coordination. Phys Ther. 2006 Aug;86(8):1151-60.

[51] Chiel HJ, Beer RD. The brain has a body: adaptive behavior emerges from interactions of nervous system, body and environment. Trends Neurosci. 1997 Dec;20(12):553-7.

[52] Ting LH, McKay JL. Neuromechanics of muscle synergies for posture and movement. Curr Opin Neurobiol. England2007:622-8.

[53] Ivanenko YP, Poppele RE, Lacquaniti F. Five basic muscle activation patterns account for muscle activity during human locomotion. J Physiol. 2004 Apr 1;556(Pt 1):267-82. 
[54] Neptune RR, Clark DJ, Kautz SA. Modular control of human walking: a simulation study. J Biomech. 2009 Jun;42(9):1282-7.

[55] Cappellini G, Ivanenko YP, Poppele RE, Lacquaniti F. Motor patterns in human walking and running. J Neurophysiol. 2006 Jun;95(6):3426-37.

[56] Chvatal SA, Ting LH. Common muscle synergies for balance and walking. Front Comput Neurosci. $2013 ; 7: 48$.

[57] Cheung VC, d'Avella A, Tresch MC, Bizzi E. Central and sensory contributions to the activation and organization of muscle synergies during natural motor behaviors. J Neurosci. 2005 Jul 6;25(27):6419-34.

[58] Danner SM, Hofstoetter US, Freundl B, Binder H, Mayr W, Rattay F, et al. Human spinal locomotor control is based on flexibly organized burst generators. Brain. 2015 Jan 12.

[59] Clark DJ, Ting LH, Zajac FE, Neptune RR, Kautz SA. Merging of healthy motor modules predicts reduced locomotor performance and muscle coordination complexity post-stroke. J Neurophysiol. 2010 Feb;103(2):844-57.

[60] Lum PS, Burgar CG, Shor PC. Evidence for strength imbalances as a significant contributor to abnormal synergies in hemiparetic subjects. Muscle Nerve. 2003 Feb;27(2):211-21.

[61] Cruz TH, Dhaher YY. Evidence of abnormal lower-limb torque coupling after stroke: an isometric study. Stroke. 2008 Jan;39(1):139-47.

[62] Sist B, Fouad K, Winship IR. Plasticity beyond peri-infarct cortex: spinal up regulation of structural plasticity, neurotrophins, and inflammatory cytokines during recovery from cortical stroke. Exp Neurol. 2014 Feb;252:47-56.

[63] Knikou M. Neural control of locomotion and training-induced plasticity after spinal and cerebral lesions. Clin Neurophysiol. 2010 Oct;121(10):1655-68.

[64] Knikou M. Plasticity of corticospinal neural control after locomotor training in human spinal cord injury. Neural Plast. 2012;2012:254948.

[65] Nudo RJ. Adaptive plasticity in motor cortex: implications for rehabilitation after brain injury. J Rehabil Med. 2003 May(41 Suppl):7-10.

[66] Nudo RJ, Milliken GW, Jenkins WM, Merzenich MM. Use-dependent alterations of movement representations in primary motor cortex of adult squirrel monkeys. J Neurosci. 1996 Jan;16(2):785-807.

[67] Martinez M, Delivet-Mongrain H, Leblond H, Rossignol S. Effect of locomotor training in completely spinalized cats previously submitted to a spinal hemisection. J Neurosci. 2012 Aug 8;32(32):10961-70.

[68] Nadeau SE, Wu SS, Dobkin BH, Azen SP, Rose DK, Tilson JK, et al. Effects of task-specific and impairmentbased training compared with usual care on functional walking ability after inpatient stroke rehabilitation: LEAPS Trial. Neurorehabil Neural Repair. 2013 May;27(4):370-80.

[69] Wirz M, Zemon DH, Rupp R, Scheel A, Colombo G, Dietz V, et al. Effectiveness of automated locomotor training in patients with chronic incomplete spinal cord injury: a multicenter trial. Arch Phys Med Rehabil. 2005 Apr;86(4):672-80.

[70] Mehrholz J, Pohl M, Elsner B. Treadmill training and body weight support for walking after stroke. Cochrane Database Syst Rev. 2014;1:Cd002840.

[71] Plummer P, Behrman AL, Duncan PW, Spigel P, Saracino D, Martin J, et al. Effects of stroke severity and training duration on locomotor recovery after stroke: a pilot study. Neurorehabil Neural Repair. 2007 Mar-Apr;21(2):137-51.

[72] Hornby TG, Campbell DD, Kahn JH, Demott T, Moore JL, Roth HR. Enhanced gait-related improvements after therapist- versus robotic-assisted locomotor training in subjects with chronic stroke: a randomized controlled study. Stroke. 2008 Jun;39(6):1786-92.

[73] Alkjaer T, Larsen PK, Pedersen G, Nielsen LH, Simonsen EB. Biomechanical analysis of rollator walking. Biomed Eng Online. 2006;5:2.

[74] Ajemian S, Thon D, Clare P, Kaul L, Zernicke RF, Loitz-Ramage B. Cane-assisted gait biomechanics and electromyography after total hip arthroplasty. Arch Phys Med Rehabil. 2004 Dec;85(12):1966-71.

[75] Maguire C, Sieben JM, Frank M, Romkes J. Hip abductor control in walking following stroke -- the immediate effect of canes, taping and TheraTogs on gait. Clin Rehabil. 2010 Jan;24(1):37-45. 

Chapter

\section{Hip abductor control in walking following stroke - the immediate effect of canes, taping and TheraTogs on gait.}

Clare Maguire, Judith M Sieben, Matthias Frank, Jacqueline Romkes.

Clinical Rehabilitation 2010; 24: 37-45. 


\section{ABSTRACT}

Objective: To confirm previous findings that hip abductor activity measured by electromyography (EMG) on the side contralateral to cane use is reduced during walking in stroke patients. To assess whether an orthosis (TheraTogs) or hip abductor taping increase hemiplegic hip abductor activity compared with activity during cane walking or while walking without aids. To investigate the effect of each condition on temporospatial gait parameters.

Design: Randomized, within-participant experimental study.

Setting: Gait laboratory.

Subjects: Thirteen patients following first unilateral stroke.

Intervention: Data collection over six gait cycles as subjects walked at self-selected speed during: baseline (without aids) and in randomized order with (1) hip abductor taping, (2) TheraTogs, (3) cane in non-hemiplegic hand.

Main measures: Peak EMG of gluteus medius and tensor fascia lata and temporo-spatial gait parameters.

Results: Cane use reduced EMG activity in gluteus medius from baseline by $21.86 \%$. TheraTogs increased it by $16.47 \%$ (change cane use-TheraTogs P $1 / 40.001$, effect size $1 / 4 \_0.5$ ) and tape by $5.8 \%$ (change cane use-tape P1/40.001, effect size $1 / 4 \_0.46$ ). In tensor fascia lata cane use reduced EMG activity from baseline by $19.14 \%$. TheraTogs also reduced EMG activity from baseline by $1.10 \%$ (change cane use-TheraTogs P1/40.009, effect size_0.37) and tape by 3\% (not significant). Gait speed (m/s) at: baseline 0.44 , cane use 0.45 , tape

0.48 , TheraTogs 0.49 .

Conclusion: Hip abductor taping and TheraTogs increase hemiplegic hip abductor activity and gait speed during walking compared with baseline and cane use. 


\section{INTRODUCTION}

Regaining the ability to walk independently is an important aim of patients and therapists in stroke rehabilitation. Canes are commonly provided during the early stages of rehabilitation to enable independent walking, although studies have consistently shown a significant reduction in surface electromyography (EMG) activity in all muscle groups on the side contralateral to cane use in both stroke and non-stroke patients. [1-4] Early walking is an important aim of stroke rehabilitation for many reasons, including the psychological well-being of the patient, to prevent loss of cardiovascular fitness, the development of secondary muscle weakness or further deterioration of abnormal muscle tone. This study questions whether canes are the optimal walking aid to enable early independent walking as their use inhibits rather than stimulates activity of the hemiplegic musculature, contrary to the aims of rehabilitation. On the positive side, canes have been shown to improve standing balance in patients with stroke by increasing the base of support.[5, 6] Positive improvements in temporo-spatial gait parameters have also been recorded. [7] Cane use provided increased stability during the single limb support phase of the hemiplegic limb, allowing more time for the contralateral (non-hemiplegic) swing phase. This resulted in increased stride period and stride length of the nonparetic limb and reduced temporal asymmetry. The authors hypothesize, however, that in the light of recent theories of motor learning, once the goal of improved stability in standing and walking is achieved through cane use, the imperative to recover inherent balance mechanisms may be educed.[8-10] Reduced balance is closely associated with low ambulatory level in community dwelling chronic stroke patients and may be an important factor in loss of cardiovascular fitness. [11] If the recovery of independent balance is adversely affected by cane use, there may be long-term consequences for the level of independence reached and for the cost of long-term care for stroke patients. To date, no studies have investigated alternatives to cane use which enable early independent walking without reducing muscle activity or providing external balance support. This study aimed to investigate the immediate effect of two alternatives TheraTogs (an elasticized orthotic garment and strapping system) and hip abductor taping - on the activity of the hemiplegic hip abductor musculature, specifically gluteus medius and tensor fascia lata, as measured by surface EMG in patients recovering from first ever stroke. The level of activity was compared to EMG activity measured when walking without walking aids (baseline) and when walking with a cane. The immediate effect of each intervention on temporo-spatial gait parameters was also assessed. The hip abductor musculature was investigated because improved postural control at the hemiplegic hip including hip abductor muscle control is an important element in restoring independent gait and balance. [12-14] It has also been shown that cane use in the contralateral hand reduces the mean hip abductor EMG by $31 \%$. This can increase to a reduction of $42 \%$ when maximum push is applied to the cane. 3 It was hypothesized that EMG activity would be reduced during cane walking compared with walking without 
aids and that EMG activity would be increased during walking with TheraTogs and tape compared with walking without aids. It was also hypothesized that temporo-spatial gait parameters would improve during cane walking compared with walking without aids and that values during TheraTogs and tape walking would also improve but less than during cane walking.

\section{METHODS}

Following approval from the Ethics Committee of Basel a randomized, within-subject experimental study design was used. Thirteen patients with hemiplegia following a first unilateral stroke were recruited from the Neurological Rehabilitation Department of the Felix-Platter Hospital in Basel, Switzerland. All patients scored at least level 3 on the Functional Ambulation Category [15] (able to walk unaided on even ground for at least $10 \mathrm{~m}$ but requiring verbal prompts and stand-by help without body contact). All patients were independent walkers prior to insult, had a Mini Mental State [16] score of 22 or above and had no orthopaedic or other neurological conditions that could limit walking ability. Patients had no gross visuospatial or visual field deficits and had no medical contraindications to walking. At baseline, descriptive variables for each patient, including height and weight, were recorded. Hip abductor muscle strength on the hemiplegic and non-hemiplegic sides was recorded while lying in a supine position using a handheld dynamometer [17] (C.I.T. Technics BV, Haren/Groningen, The Netherlands). Skin was then prepared for surface EMG placement. The skin was shaved over the gluteus medius and tensor fascia lata muscles and rigorously cleaned with alcohol. Surface EMG electrodes were placed onto the skin overlying these muscles following the 'European Recommendations for Surface Electromyography"(SENIAM) guidelines. [18] The ground electrode was placed on the clean-shaven skin over the sacrum. Bipolar $\mathrm{Ag} / \mathrm{AgCl}$ surface electrode pairs with an electrode diameter of $10 \mathrm{~mm}$ and an inter-electrode spacing of $22 \mathrm{~mm}$ were used. Using a Neurodata system (Vienna, Austria; amplifiers of Biovison, Wehrheim, Germany) at a sampling rate of $2520 \mathrm{~Hz}$, EMG signals were pre-amplified and band-pass filtered $(10-700 \mathrm{~Hz})$. Skin impedance between the electrodes was measured with a skin impedance monitor (Grass Electrode Impedance Meter, Astro-Med, Inc, W.Warwick, RI, USA). An impedance range of 1-10 kohm was considered acceptable.[19]19 Gait parameters were assessed using a sixcamera, $120 \mathrm{~Hz}$ VICON 460 motion measurement system (Oxford Metrics Ltd, Oxford, UK). Fourteen-millimeter-diameter sphere-shaped reflective markers were affixed with double-sided tape bilaterally to the calcaneus, lateral malleolus, second metatarsal head and lateral surface of the lower leg below the head of the fibula. Infrared sensitive solid-state cameras were used within this system for locating and following the fixed reflective markers through space. Patients were asked to walk at a self-selected speed over an indoor, hard surface 10-m walkway without walking aids in their own footwear and with usual foot-ankle orthoses. 
Patients were accompanied by an assistant at all times who remained within arms' reach to ensure safety. EMG and temporo-spatial parameters were collected to establish pre-experimental baseline values while walking without aids until six gait cycles with clear data-sets were collected. A gait cycle was defined as starting with initial foot contact and ending with the subsequent foot contact of the same leg. Testing of the remaining three conditions then continued in a randomized order determined by a computerized randomization program while walking with (2) hip abductor taping, (3) TheraTogs, (4) cane with cane at normal height (defined as at the level of the radial styloid of the sound wrist) - at the Movement Analysis Laboratory, Basel, Switzerland.

Taping was applied to the hemiplegic side with the patient standing with the hip in 5 degrees abduction. Three bands of taping were applied from just below the greater trochanter (1) directly upwards to the iliac crest, (2) upwards and anteriorally to the anterior superior iliac spine, (3) upwards and posteriorally towards the posterior third of the iliac crest. The TheraTogs consisted of two wide straps which were applied under tension over a hip held in 5 degrees abduction in standing, spanning the thigh cuff, crossing over the hip joint and attaching to the torso above the iliac crest. All conditions are depicted in Figure 1.

\section{Primary outcome measure}

Percentage change of peak EMG in millivolts from baseline while walking with cane, TheraTogs and tape for gluteus medius and tensor fascia lata.

Secondary outcome measures

Temporo-spatial gait parameters at baseline and during interventions:

- $\quad$ self-selected walking speed in $\mathrm{m} / \mathrm{s}$,

- spatial asymmetry - calculated as step length

- asymmetry ratio (defined as non-paretic step length $(m) /$ paretic step length $(m)$ ), and

- temporal asymmetry - calculated as mean single limb support time ratio (defined as mean non-paretic single limb support time (s)/ mean paretic single limb support time (s)).

These measures have been shown to correlate with balance and degree of motor function of the lower extremities following stroke. $[20,21]$ 


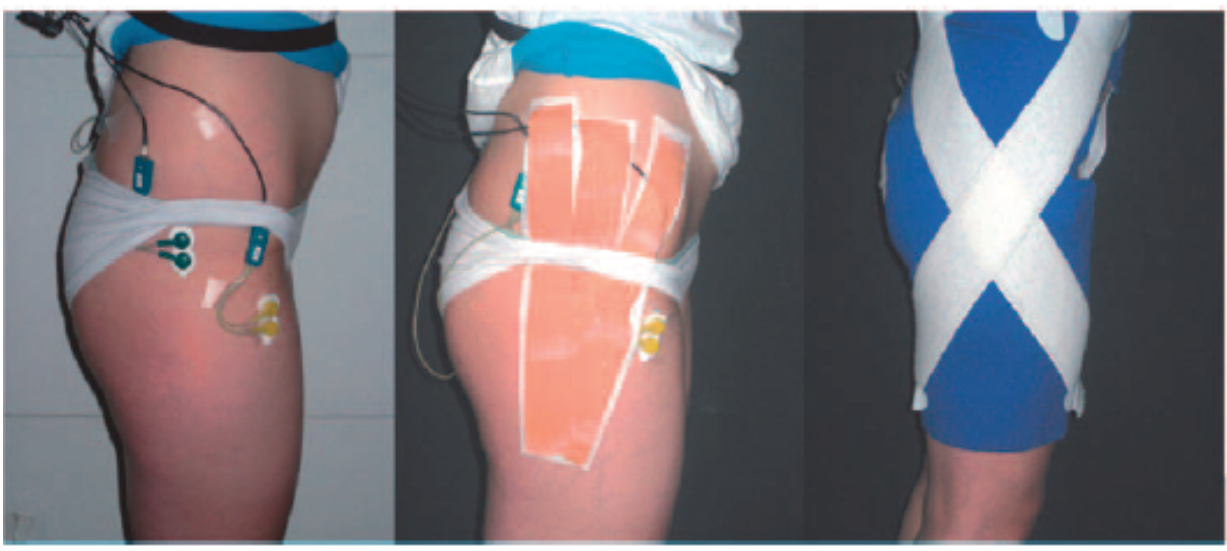

Figure 1. Surface electrode positions, hip abductor taping technique and TheraTogs application.

The EMG signals were full-wave rectified and each data-set of one gait cycle was interpolated to 300 data-points using the MATLAB software package. For each subject the mean of each data-point from six gait cycles was calculated for each condition separately (baseline and interventions) and for each muscle (gluteus medius and tensor fascia lata). The peak EMG amplitude during baseline and interventions for each muscle was then identified. The EMG values obtained were then compared as a percentage change from baseline. Friedman's ANOVA was used for non-parametric repeated measures analysis of variance to test for statistically significant differences in percentage change of peak EMG values between interventions, baseline and cane use. Wilcoxon tests were used as post-hoc tests to follow-up these findings and a Bonferroni correction was applied. All effects are therefore reported at a 0.0167 level of significance. Friedman's ANOVA was also used to test for significant differences between changes in gait speed.

\section{RESULTS}

Thirteen subjects with hemiplegia following a first unilateral stroke were recruited. Five of the subjects were women and six had a right-sided hemiplegia. Mean age was 64 (standard deviation 14). Twelve of the 13 patients had just reached the point in their rehabilitation where they were to be provided with a cane or rollator frame to enable independent walking. The mean time since stroke for these patients was 9.2 weeks (range 5-16 weeks, standard deviation 3.8 weeks). These patients were able to walk short distances independently (minimum $10 \mathrm{~m}$ ) but all required stand by assistance for safety when walking without aids. Three subjects wore ankle-foot orthoses due to inadequate dorsiflexion for foot clearance. One subject had a more chronic stroke (64 weeks 
since stroke) was independently mobile with a cane, and could walk on even surfaces independently without aids for approx. $30 \mathrm{~m}$.

EMG data for each muscle and each condition was processed and graphically represented as in Figure 2. EMG data collected and temporo-spatial gait parameters are presented in Table 1.

Friedman's ANOVA showed statistically significant differences between baseline and interventions for gluteus medius (chi-square (2) 1/419.077, P50.001) and tensor fascia lata (chi-square (2) 1/47.538, P1/40.025). Cane use reduced EMG activity for gluteus medius from baseline by $21.86 \%$ whereas TheraTogs increased EMG activity for gluteus medius from baseline by $16.47 \%$. This represented a highly statistically significant difference for change in peak EMG between cane use and TheraTogs for gluteus medius (P50.001).

EMG channel 1
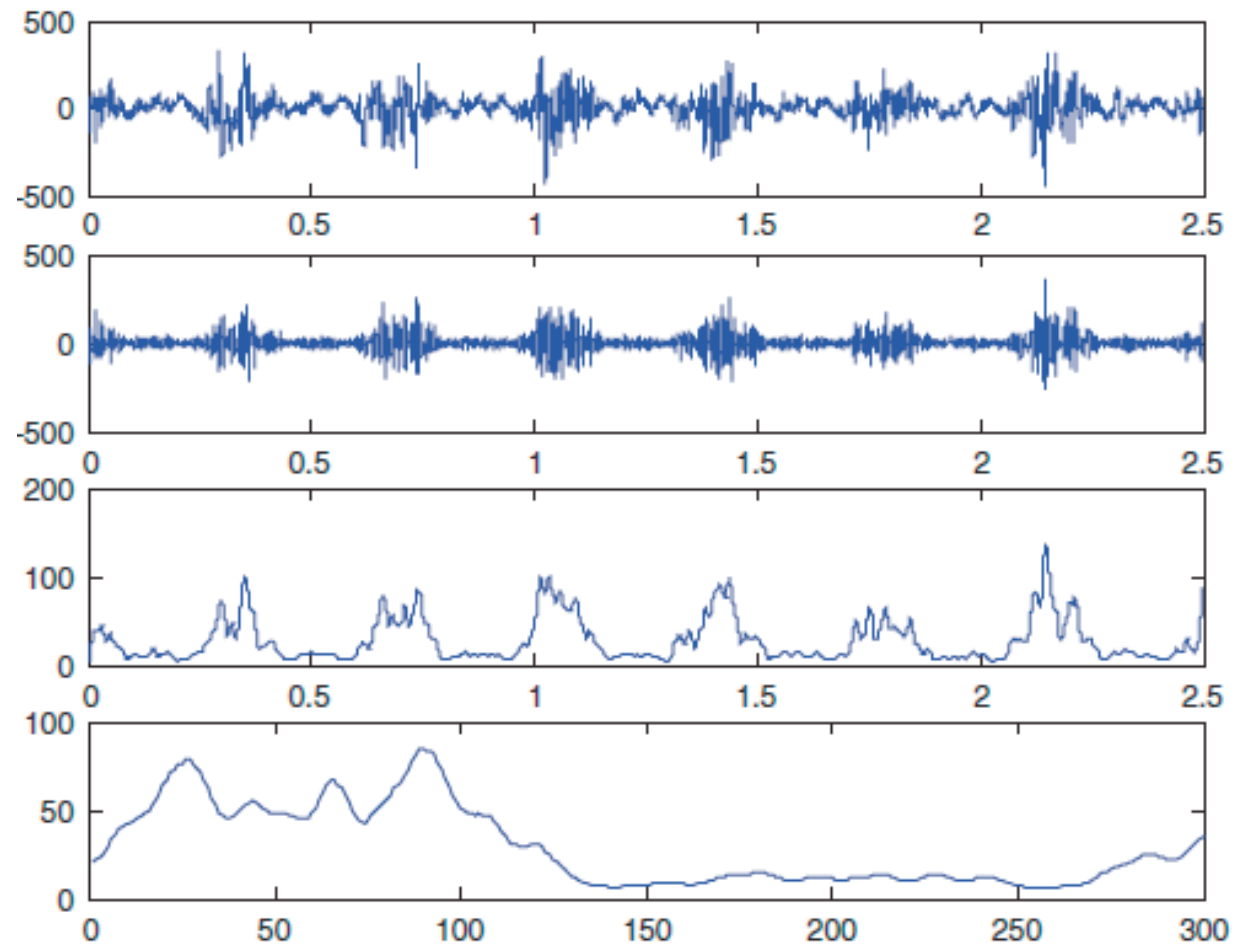

Figure 2. A typical representation of raw and smooth rectified surface EMG of baseline walking for gluteus medius muscle. Each block represents in ascending order: (1) raw EMG recording, (2) high and low pass filtered, (3) full wave rectified and smoothed, (4) one selected gait cycle of data. 
Table 1. EMG values and temporo-spatial gait parameters

\begin{tabular}{lcccc}
\hline & Baseline & $\begin{array}{c}\text { Walking with } \\
\text { cane }\end{array}$ & $\begin{array}{c}\text { Walking with } \\
\text { tape }\end{array}$ & $\begin{array}{c}\text { Walking with } \\
\text { TheraTogs }\end{array}$ \\
$\begin{array}{l}\text { Mean \% change of EMG for gluteus medius } \\
\text { between baseline and intervention. (SD) }\end{array}$ & - & $-21.8(19.3)$ & $5.8(28.2)$ & $16.4(23.6)$ \\
Mean EMG mV (SD) gluteus medius & $76(37)$ & $56(31)$ & $78(39)$ & $87(47)$ \\
$\begin{array}{l}\text { Median EMG mV gluteus medius } \\
\text { Mean \% change of EMG for tensor fascia }\end{array}$ & -82 & 61 & 87 & 107 \\
$\begin{array}{l}\text { lata between baseline and intervention (SD) } \\
\text { Mean EMG mV (SD) tensor fascia lata }\end{array}$ & $99(77)$ & $74(61)$ & $87(60)$ & $91(62)$ \\
$\begin{array}{l}\text { Median EMG mV tensor fascia lata } \\
\text { Mean gait speed m/s (SD) }\end{array}$ & $8.44(0.23)$ & $0.45(0.17)$ & $0.48(0.20)$ & 96 \\
$\begin{array}{l}\text { Mean step length asymmetry ratio (spatial } \\
\text { asymmetry) }\end{array}$ & $1.27(0.87)$ & $0.91(0.29)$ & $1.08(0.30)$ & $0.93(0.26)$ \\
$\begin{array}{l}\text { Mean single limb support time ratio } \\
\text { (temporal asymmetry) }\end{array}$ & $1.24(0.41)$ & $1.15(0.25)$ & $1.30(0.55)$ & $1.33(0.47)$
\end{tabular}

${ }^{a}$ Values41 represent longer step length with non-hemiplegic leg and vice versa.

${ }^{b}$ Values41 represent longer single support time on non-hemiplegic leg

The effect size of TheraTogs use was - 0.5 (large effect size by Cohen's benchmark). Tape increased EMG activity from baseline by $5.8 \%$. This change between cane use and tape was also statistically significant $(P=0.001)$ and the effect size -0.46 was medium to large. No statistically significant difference was identified between TheraTogs and tape use $(P=0.029)$, although a small to medium effect size was calculated -0.30 in favour of TheraTogs use. For tensor fascia lata, cane use reduced EMG activity from baseline by 19.14\%. In this instance TheraTogs also reduced EMG activity from baseline by $1.10 \%$. This represented a statistically significant difference between percentage change in peak EMG between cane use and TheraTogs ( $P=0.009)$. The effect size -0.37 was medium. EMG activity during tape walking was reduced by $3 \%$. This difference between cane use and tape was not statistically significant. No significant difference between TheraTogs and tape was identified for tensor fascia lata. Gait speed improved with TheraTogs and tape compared to baseline and cane walking althoughno statistically significant differences were found. Step length asymmetry was greatest at baseline and was reduced during cane use and interventions.

Symmetry was greatest with TheraTogs followed by tape then cane. Cane use and TheraTogs led to longer step lengths with the hemiplegic leg than the non-hemiplegic leg. Taping resulted in non-hemiplegic step lengths remaining longer than hemiplegic, as was the case at baseline, although the difference was reduced. Temporal asymmetry was reduced from baseline only during cane use and was increased with both taping and TheraTogs. At baseline single support on the non-hemiplegic leg was longer than on the hemiplegic leg. This tendency was increased with TheraTogs and taping. Cane use led to increased single support on the hemiplegic leg. 


\section{GRAPHICAL REPRESENTATION}

Processed EMG data was averaged for six gait cycles. The resulting mean EMG values were calculated for one gait cycle for both muscles during all interventions. Figures 3 and 4 graphically represent these values for one representative patient. In Figure 3 mean EMG for cane use lies below the mean EMG for baseline throughout the majority of the gait cycle. Mean EMG values for tape and TheraTogs are higher than mean EMG for baseline throughout the gait cycle and peak EMGs for tape and TheraTogs are both clearly increased from baseline.
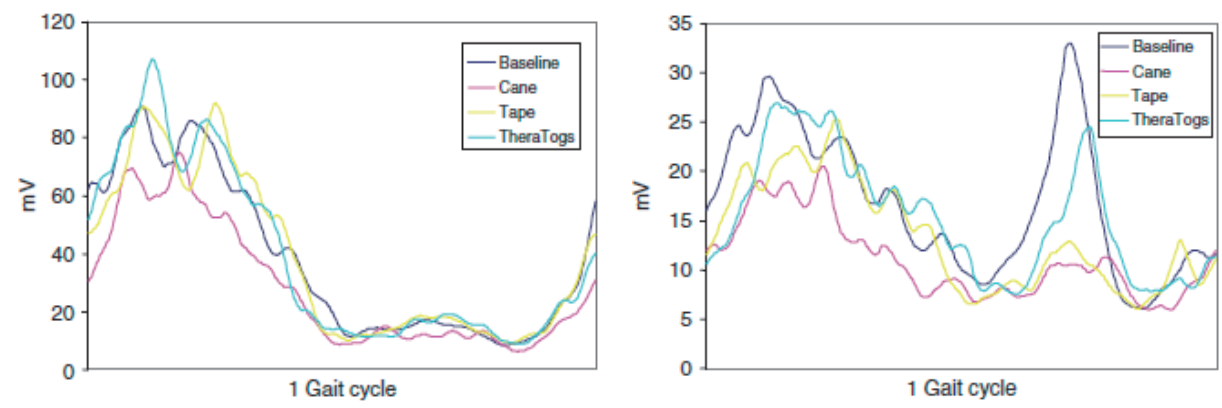

Figure 3. Smooth rectified mean EMG graphs of glute- Figure 4. Smooth rectified mean EMG graphs of the us medius muscle of a representative subject walking tensor fascia lata muscle of a representative subject for one gait cycle (initial foot contact to subsequent walking. foot contact) at baseline and during interventions.

In Figure 4 mean EMG for cane use lies clearly below the mean EMG for baseline throughout the gait cycle. Mean EMGs for tape and TheraTogs show a tendency to lie between these two values with TheraTogs EMG values generally higher than tape and increased beyond baseline at some points.

\section{POSSIBLE EFFECT MODIFIERS}

Pearson correlations were calculated for each muscle to see whether results were influenced by body mass index (BMI), time since stroke or variations in hip abductor muscle strength ratio.

No statistically significant correlations were calculated. 


\section{DISCUSSION}

The results of this investigation confirm the findings of other studies in both stroke and non-stroke subjects1-4 that the EMG activity of hip abductor muscles is significantly reduced during cane walking, on the side contralateral to cane use. This study has shown, for the first time, that alternative treatment interventions are available which have the immediate effect of significantly increasing hip abductor muscle activity in stroke patients during walking as in the case of the gluteus medius, or have a significantly reduced negative effect as in the case of tensor fascia lata. Two of the three temporo-spatial gait parameters measured - gait speed and spatial asymmetry - were improved with TheraTogs and tape compared with baseline and cane walking. One parameter, temporal asymmetry, was worsened with TheraTogs and taping compared with baseline and cane walking. Kirker et al. [12] have shown that hip abductor activity post stroke is primarily disrupted while initiating movement (e.g. taking a first step) and when responding to external perturbations. These abilities are important for independent walking at home and in the community. Interventions such as taping and TheraTogs which improve hip abductor activity during these movements may be more effective in promoting recovery than interventions which consistently reduce activity, as in cane use. These conclusions are supported by Kim and Eng [14] in a study to investigate the relationship between walking speed in stroke survivors and kinemetic and kinetic gait profiles.

This study concluded that interventions which increase frontal plane hip powers by strengthening the hemiplegic hip abductors would increase gait speed. Gait speed was increased with the interventions although no statistically significant differences were found between speeds and all gait speeds remained within the same functional category. [22, 23] Schmid et al.[22] and Perry et al. [23] determined that household ambulation is equivalent to severe gait impairments and a walking speed of $50.4 \mathrm{~m} / \mathrm{s}$. Moderate gait impairments are equivalent to between 0.4 and $0.8 \mathrm{~m} / \mathrm{s}$ and represent limited community ambulation. Full community ambulation indicates mild impairment and a walking speed of $40.8 \mathrm{~m} / \mathrm{s}$. In this study gait speed always remained within the middle category of moderate gait impairments $(0.4-0.8 \mathrm{~m} / \mathrm{s})$. Although gait speed improved, neither treatment interventions nor cane use had the immediate effect of moving gait speed to a different functional category. Step length asymmetry ratios showed that spatial symmetry was improved compared to baseline with both interventions and dur-

ing cane use. However temporal asymmetry ratios were improved only during cane use and were increased, showing more asymmetry compared to baseline, with both interventions. As the temporo-spatial gait parameters of walking speed and symmetry have been shown to correlate with balance and lower limb function following stroke [21, 22, $24,25]$, the immediate improvement of two from three of these parameters may indicate that balance reactions are also stimulated during walking with taping and TheraTogs. The use of surface EMG electrodes to assess several interventions, two of 
which cover the electrodes, could have been problematic. The quality of the EMG signal could have been compromised. Through visual assessment of the raw EMG signal between interventions, and by measurement of skin impedance at the beginning and end of each trial, signal quality was assured One further drawback of the present study is the lack of information gained about the longer term effects of the interventions. Although the aim of the study was to assess the immediate effects of the interventions, clinical relevance cannot be assumed until these effects have been investigated over a longer time period. The results of this study indicate that cane use may not be optimal during the early stages of gait rehabilitation following stroke. TheraTogs and taping may be more effective in promoting muscle activity and balance reactions. Clinical trials to assess and compare the long-term effects of the interventions and cane use are needed.

\section{CLINICAL MESSAGES}

- Cane walking following stroke reduces hemiplegic hip abductor muscle activity compared with walking without walking aids.

- Hip abductor taping and TheraTogs increase hemiplegic hip abductor activity and gait speed during walking compared with walking without aids or walking with a cane.

\section{ACKNOWLEDGEMENTS}

We would like to thank Georg Ferber for his statistical advice and assistance. We would also like to thank Alfio Albasini for his advice relating to taping techniques.

\section{SOURCE OF EQUIPMENT}

TheraTogs, Inc., Corporate Offices, 305 Society drive, Suite C-3, Telluride, Colorado 81435-8914, USA. TheraTogs orthoses were provided free of charge for research purposes from the above named company. No other financial support was or provided by this or any other company. 


\section{REFERENCES}

[1] Buurke JH, Hermens HJ, Erren-Wolters CV, Nene AV. The effect of walking aids on muscle activation patterns during walking in stroke patients. Gait Posture. 2005 Oct;22(2):164-70.

[2] Ajemian S, Thon D, Clare P, Kaul L, Zernicke RF, Loitz-Ramage B. Cane-assisted gait biomechanics and electromyography after total hip arthroplasty. Arch Phys Med Rehabil. 2004 Dec;85(12):1966-71.

[3] Neumann DA. An electromyographic study of the hip abductor muscles as subjects with a hip prosthesis walked with different methods of using a cane and carrying a load. Phys Ther. 1999 Dec;79(12):1163-73; discussion 74-6.

[4] Neumann DA. Hip abductor muscle activity as subjects with hip prostheses walk with different methods of using a cane. Phys Ther. 1998 May; 78(5):490-501.

[5] Laufer Y. Effects of one-point and four-point canes on balance and weight distribution in patients with hemiparesis. Clin Rehabil. 2002 Mar;16(2):141-8.

[6] Laufer $Y$. The effect of walking aids on balance and weight-bearing patterns of patients with hemiparesis in various stance positions. Phys Ther. 2003 Feb;83(2):112-22.

[7] Kuan TS, Tsou JY, Su FC. Hemiplegic gait of stroke patients: the effect of using a cane. Arch Phys Med Rehabil. 1999 Jul;80(7):777-84.

[8] Mastos M, Miller K, Eliasson AC, Imms C. Goal-directed training: linking theories of treatment to clinical practice for improved functional activities in daily life. Clin Rehabil. 2007 Jan;21(1):47-55.

[9] Krakauer JW. Motor learning: its relevance to stroke recovery and neurorehabilitation. Curr Opin Neurol. 2006 Feb;19(1):84-90.

[10] Huang VS, Krakauer JW. Robotic neurorehabilitation: a computational motor learning perspective. J Neuroeng Rehabil. 2009;6:5.

[11] Michael KM, Allen JK, Macko RF. Reduced ambulatory activity after stroke: the role of balance, gait, and cardiovascular fitness. Arch Phys Med Rehabil. 2005 Aug;86(8):1552-6.

[12] Kirker SG, Simpson DS, Jenner JR, Wing AM. Stepping before standing: hip muscle function in stepping and standing balance after stroke. J Neurol Neurosurg Psychiatry. 2000 Apr;68(4):458-64.

[13] Kirker SG, Jenner JR, Simpson DS, Wing AM. Changing patterns of postural hip muscle activity during recovery from stroke. Clin Rehabil. 2000 Dec;14(6):618-26.

[14] Kim CM, Eng JJ. Magnitude and pattern of 3D kinematic and kinetic gait profiles in persons with stroke: relationship to walking speed. Gait Posture. 2004 Oct;20(2):140-6.

[15] Holden MK, Gill KM, Magliozzi MR, Nathan J, Piehl-Baker L. Clinical gait assessment in the neurologically impaired. Reliability and meaningfulness. Phys Ther. 1984 Jan;64(1):35-40.

[16] Folstein MF, Folstein SE, McHugh PR. "Mini-mental state". A practical method for grading the cognitive state of patients for the clinician. J Psychiatr Res. 1975 Nov;12(3):189-98.

[17] Click Fenter P, Bellew JW, Pitts TA, Kay RE. Reliability of stabilised commercial dynamometers for measuring hip abduction strength: a pilot study. Br J Sports Med. 2003 Aug;37(4):331-4.

[18] Hermens HJ, Freriks B, Disselhorst-Klug C, Rau G. Development of recommendations for SEMG sensors and sensor placement procedures. J Electromyogr Kinesiol. 2000 Oct;10(5):361-74.

[19] Konrad P. The ABC of EMG. A Practical Introduction to Kinesiological Electromyography.: Noroxon 2006.

[20] Nadeau S, Arsenault AB, Gravel D, Bourbonnais D. Analysis of the clinical factors determining natural and maximal gait speeds in adults with a stroke. Am J Phys Med Rehabil. 1999 Mar-Apr;78(2):123-30.

[21] Brandstater ME, de Bruin H, Gowland C, Clark BM. Hemiplegic gait: analysis of temporal variables. Arch Phys Med Rehabil. 1983 Dec;64(12):583-7.

[22] Schmid A, Duncan PW, Studenski S, Lai SM, Richards L, Perera S, et al. Improvements in speed-based gait classifications are meaningful. Stroke. 2007 Jul;38(7):2096-100.

[23] Perry J, Garrett M, Gronley JK, Mulroy SJ. Classification of walking handicap in the stroke population. Stroke. 1995 Jun;26(6):982-9.

[24] Patterson KK, Parafianowicz I, Danells CJ, Closson V, Verrier MC, Staines WR, et al. Gait asymmetry in community-ambulating stroke survivors. Arch Phys Med Rehabil. 2008 Feb;89(2):304-10. 
Hip abductor control and walking aids post-stroke

[25] Witte US, Carlsson JY. Self-selected walking speed in patients with hemiparesis after stroke. Scand J Rehabil Med. 1997 Sep;29(3):161-5. 



\section{Chapter}

\section{Walking with a four wheeled walker (rollator) significantly reduces EMG lower-limb muscle activity in healthy subjects.}

Zorica Suica, Jacqueline Romkes, Amir Tal, PT, Clare Maguire 


\section{ABSTRACT}

Objective: To investigate the immediate effect of four-wheeled- walker(rollator)walking on lower-limb muscle activity and trunk-sway in healthy subjects.

Methods: In this cross-sectional design electromyographic(EMG) data was collected in six lower-limb muscle groups and trunk-sway was measured as peak-to-peak angular displacement of the centre-of-mass (level L2/3) in the sagittal and frontal-planes using the SwayStar balance system. 19 subjects walked at self-selected speed firstly without a rollator then in randomised order 1 . with rollator 2 . with rollator with increased weightbearing.

Results: Rollator-walking caused statistically significant reductions in EMG activity in lower-limb muscle groups and effect-sizes were medium to large. Increased weightbearing increased the effect. Trunk-sway in the sagittal and frontal-planes showed no statistically significant difference between conditions.

Conclusion: Rollator-walking reduces lower-limb muscle activity but trunk-sway remains unchanged as stability is likely gained through forces generated by the upper-limbs. Short-term stability is gained but the long-term effect is unclear and requires investigation.

\section{Keywords}

Assistive devices, rollator, wheeled-walker, EMG, trunk stability 


\section{INTRODUCTION}

Rollators are four-wheeled walkers commonly used in clinical practice in many patient groups (Gupta et al., 2006; Chee et al., 2013; Eggermont et al., 2006; Smith et al., 2012). The specific aims of rollator use vary depending on the patient diagnosis. For example, in the elderly or patients with neurological disease such as multiple sclerosis, rollators are used to increase walking endurance and muscle strength or to improve balance (Chee et al., 2013; Vogt et al., 2010; Braun et al., 2014 ). Following surgery, reduced weight bearing or pain may be treatment goals (Smith et al., 2012), whereas in patients with chronic obstructive pulmonary disease improvement of functional exercise capacity and reduction of dyspnoea can be the objectives (Gupta et al., 2006).

To optimally prescribe rollators therefore, clear treatment goals for each patient are necessary as well as a thorough understanding of the biomechanical and physiological effects of rollator walking.

Despite this, the biomechanical effects have only been sparsely investigated. Alkjaer et al.( 2006) assessed sagittal and frontal plane kinematics and joint dynamics in the lower limbs of seven healthy women walking with and without rollators. They concluded that walking with a rollator unloaded the knee and ankle extensors (which would reduce extensor muscle activity at these joints although this was not directly measured), whilst increasing hip extensor load (which would suggest increased hip extensor muscle activity).

Ishikura (2001) did investigate muscle activity with surface electromyography (EMG) when walking with walker at varying degrees of hip flexion. This study concluded that muscle activity in both the rectus femoris and biceps femoris was reduced during walker gait and that reduction in activity increased with increasing hip flexion.

To our knowledge no further studies have investigated the effect of rollator walking on muscle activation. The shortage of such studies as well as the partially contradictory conclusions of those mentioned above (Ishikura (2001) stating that muscle activity is reduced, Alkjaer et al. (2006) suggesting that it would be increased in hip extensors) mean that the influence of rollator walking on muscle activity remains unclear although this is an important consideration during clinical prescription. If muscle activity is reduced during rollator walking, long term use may reduce muscle strength. This could be relevant for patients groups in which rehabilitation aims to maintain or increase muscle strength e.g. geriatric or neurologically impaired patients. On the other hand, reduction in muscle activity may be important for some patients e.g. to unload joints following surgery. A clearer understanding of the effect of rollator walking on muscle activity would improve the appropriate and evidence based prescription of these devices.

The influence of rollators on the control of balance also remains unclear. Alkjaer et al. (2006) stated that "hip range of movement in the frontal plane was significantly less walking with a rollator than without". At the same time, the internal hip abductor moment was reduced. 
In this situation reduced abductor moment would likely be due to reduced hip abductor muscle activity (although this was not directly measured) which would cause contralateral pelvic drop during single leg stance and therefore increased movement in the frontal plane. This in turn would increase trunk sway peak to peak angular displacement of the Centre of Mass (CoM) (Allum et al., 2001) indicating balance instability (Corporaal et al., 2013; Gill et al., 2001; Goutier et al., 2010). The importance of hip abductor control in balance stability has long been established (Runge et al., 1999; Winter et al., 1996) and more recently confirmed (Gribble \& Hertel, 2004; Negahban et al., 2013).

Additionally lateral spontaneous sway amplitude was found to be the single best predictor of future risk of falling in an elderly population (Maki et al.1997).

The fact that hip range of motion in the frontal plane did not increase despite reduced hip abductor activity, suggests that other mechanisms than hip abductor muscle activity were responsible for maintaining frontal plane pelvic stability. Pardo et al. (1993) investigating rollator use in patients with lower limb amputations suggested that these stabilizing mechanisms are generated by the upper limb reaction forces and moments at the walker. This could mean that rollator use facilitates use of the arms and reduces typical patterns of muscle activation in the lower limbs to maintain stability. Compensatory, abnormal balance reactions to maintain stability may therefore be encouraged during rollator walking.

Rollators also increase the base of support (BoS) during standing and walking (Bateni \& Maki, 2005). As balance comprises maintaining the CoM within the limits of the BoS, (Bateni \& Maki, 2005; Maki \& Mcllroy, 1997; Pai \& Patton, 1997) rollator use, through increasing the BoS, makes it easier to balance.

These points would be important considerations in the prescription of rollators. When rehabilitation aims to improve balance reactions, a walking aid which does not facilitate normal reactions is possibly not optimal e.g. in fall prevention programs or in early post-stroke recovery. Alternatively, when improvement of balance control is not the priority of treatment, rather the provision of stability to enable independent mobility e.g. in some eldery patient groups, a rollator may be the appropriate device.

This study aims to investigate the effect of rollator walking with normal and increased weight bearing on muscle activity in the lower extremities and concurrently on trunk sway in comparison to walking without a rollator in healthy subjects.

We suggest that if the person pushes on the rollator toward the ground, a rollator force in the opposite rotary direction to the body weight force will be generated(see Figure 1).

The reactive rollator force will form force couples with the hip extensor, abductor and ankle plantar flexor muscle groups, the force couples being equal and opposite to the bodyweight. The contribution of muscle activity necessary to oppose the effects of body weight will therefore be reduced. Increasing push on the rollator will increase the reactive rollator force thus further reducing muscle activity. 


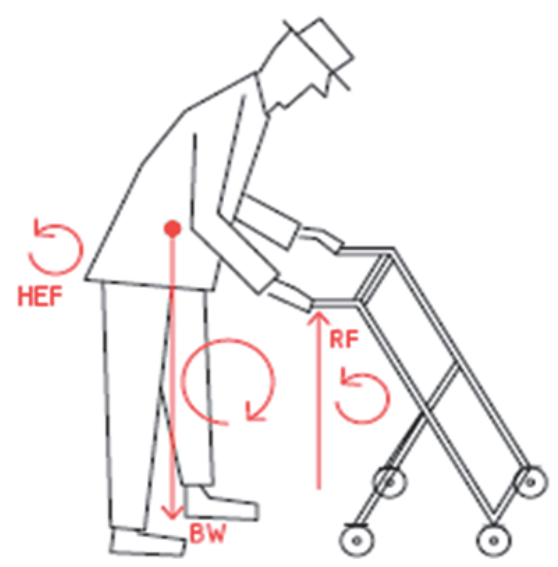

Figure 1 The hip extensor force (HEF) and rollator force (RF) combining to generate torques equal and opposite to the direction to the torque generated by the body weight force (BW).

Our first hypothesis is therefore that rollator walking will reduce hip extensor, abductor and plantar flexion muscle activity. Increasing push on the rollator will further reduce muscle activity.

Our second hypothesis is that knee extensor and ankle dorsiflexion activity will be reduced.

We suggest that this could be due to hip flexion shifting the bodyweight force forwards creating knee extension and ankle dorsiflexion moments about these joints.

If these hypotheses are true that muscle activity is reduced in the hip and lower extremity musculature during rollator walking, we further suggest that stability at the pelvis and trunk are maintained by activity and force generation of the upper extremities. Our third hypothesis is therefore that despite reduced hip extensor and abductor muscle activity, trunk sway in the sagittal and frontal planes will not increase during rollator walking.

\section{METHODS}

\section{Participants}

Following approval from the Ethics Committee of Basel nineteen healthy subjects(10 female, 9 male) age from 22 to 70 years, body mass of $70.95 \pm 8.8 \mathrm{~kg}$, and height of $172.7 \pm 9.3 \mathrm{~cm}$. with normal walking ability, no cardiovascular or neurological diseases, no lower extremity prostheses or orthoses and no diabetes were recruited. All subjects gave their written informed consent to participate in this cross-sectional study design. 


\section{Primary and secondary outcome measures}

The primary outcome measure was percentage change of surface electromyography (EMG). Peak of mean values over nine gait cycles in microvolts $(\mu \mathrm{V})$ were compared between walking without a rollator (baseline assessment) and rollator walking with a push (increased weight bearing).

Secondary outcomes were peak-to-peak angular displacement of the CoM in the frontal-plane (roll) and sagittal-plane (pitch) in degrees for each condition.

\section{Sample Size}

In order to detect a clinically significant difference in the primary outcome measure with a probability of $80 \%$ at a two sided $5 \%$ significance level a total of 14 subjects were necessary. This calculation was performed assuming a mean difference of EMG and standard deviation (SD) for gluteus medius between normal and cane gait based on the studies by (Maguire et al., 2010; Neumann, 1998).

\section{Testing Procedure}

The rollator (Figure 2) was firstly adjusted for each subject in an upright standing position with the arms hanging down along the body so that the handles were on a level with the styloid process (Alkjaer et al., 2006).

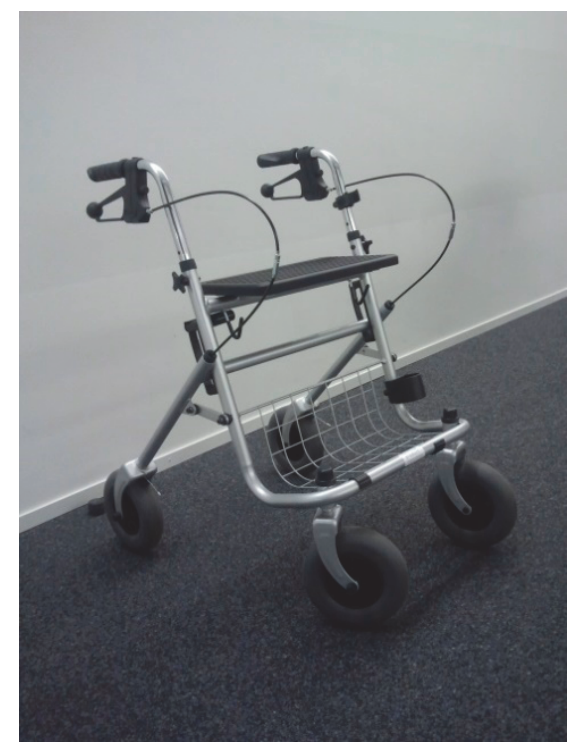

Figure 2 This Rollator, Ergo-Bremse 326 NPU, was used in the study. 
Subjects walked for approximately 5 minutes with the rollator before measurements began to familiarize themselves with rollator use.

Each subject was instructed to walk at a self-selected, comfortable speed over a level, eight meter walkway firstly without a rollator then in a computer generated randomised order (1) with a rollator (2) with a rollator with a "push" described as a "moderate but comfortable amount of pressure". EMG activity for each muscle and trunk sway were concurrently measured.

To obtain an objective measure of the subjective variable „moderate but comfortable amount of pressure" and to assess whether this was comparable between subjects, all subjects stood with the rollator wheels on four weighing scales -each wheel on one of four identical scales. Subjects were instructed to push with a "moderate but comfortable amount of pressure" -the same as whilst they were walking. The amount of weight on each wheel was measured in $\mathrm{kg}$. This was repeated three times and a mean for each wheel was calculated.

The subjective amount of weight bearing and this objective measurement thereof have been shown to correlate therefore validating this method (Malviya et al., 2005; Youdas et al., 2005).

\section{EMG Measurement system}

Surface electromyography (EMG) was used to measure muscle activity with a 16 bipolar channel Porti system (TMS International, Enschede, the Netherlands). Skin was prepared firstly shaved over the relevant muscles and then cleaned with alcohol. Noraxon bipolar $\mathrm{Ag} / \mathrm{AgCl}$ surface EMG electrode pairs with an electrode diameter of $10 \mathrm{~mm}$ and an inter-electrode spacing of $20 \mathrm{~mm}$ (Noraxon USC, Inc.) were placed on gluteus medius and maximus, rectus femoris, semitendinosus, tibialis anterior and gastrocnemius on both lower extremities following the "European Recommendations for Surface Electromyography" (SENIAM) guidelines (Hermens et al., 2000). The ground electrode was placed overlying the sacrum.

\section{EMG Data Collection}

Data was collected for each muscle over nine gait cycles as the subject walked at a selfselected, comfortable speed under the three conditions. Gait cycles were defined as heel strike to heel strike of the same leg determined by a foot switch placed mid-heel and synchronised with the measurement system.

EMG was measured at a sampling rate of $2048 \mathrm{~Hz}$ without filtering, so that the signals were measured including Direct Current (DC). The digitalised data was high pass filtered with a fourth order filter with a cut off frequency of $10 \mathrm{~Hz}$. The EMG signals were full wave rectified and each data-set of one gait cycle was interpolated to 500 data- 
points using the MATLAB software package (CThe MathWorks, Inc, 1994-2014). The data was exported to Microsoft Excel ${ }^{\circledR}$ (Vers. 14.1.0, 2011) for further analysis.

For each subject the mean of each EMG data-point over nine gait cycles was calculated in $\mu \mathrm{V}$ for each muscle and each condition separately. The peak from the mean EMG was then identified. The EMG values of conditions 1.rollator walking and 2.rollator walking with a "push" were compared as a percentage change to values when walking without rollator (baseline).

\section{Trunk Sway Data Collection}

Trunk Sway was measured when walking under each condition using the SwayStar balance system (Balance International Innovations $\mathrm{GmbH}$, Iseltwald, Switzerland), which consists of two angular velocity sensors (fibre-optic gyroscopes). SwayStar was attached via a belt to the subject at the midline of the second and third lumbar spine vertebrae (L2/3). The sensors were attached to a computer via a Bluetooth communication which sampled the velocity signals every $100 \mathrm{~ms}$ and numerically integrated the velocity signals to yield angular displacement. Trunk sway was measured as peak to peak angular displacement of the CoM (level L2/3) in the frontal plane (roll) and sagittal plane (pitch) in degrees during an $8 \mathrm{~m}$ walk for each condition.

The Sway Star has been used to assess trunk sway in healthy subjects and many pathologies and differing age groups (Allum \& Carpenter, 2005; Allum et al., 2001; Bischoff-Ferrari et al., 2006; Corporaal et al., 2013; Horlings et al., 2009).

\section{Statistical Analysis}

SPSS (IBM ${ }^{\circledR}$ SPSS $^{\circledR}$ Statistics, Version 20.0, 2011) was used for statistical analysis.

Because the EMG and Sway Star data were not normally distributed (the Kolmogorov- Smirnov significance was $\mathrm{P}<0.05)$, Friedman's ANOVA was used to test for statistically significant differences between all conditions baseline, rollator walking and rollator walking with a "push". Wilcoxon signed-rank tests were used as post-hoc tests for evaluating differences between: 1) baseline and rollator-walking and 2) baseline and rollator walking with a "push".

The effect size was calculated using the equation $r=Z / V N$ in which $r=$ effect size; $Z=$ $\mathrm{z}$-score obtained from the SPSS Output for Wilcoxon signed-rank test produces; $\mathrm{N}=$ number of total observations. To interpret the effect size Cohen's benchmarks: $r=.10$ small effect; $r=.30$ medium effect; $r=.50$ large effect was used (A, 2005). 


\section{RESULTS}

"Moderate but comfortable amount of pressure" (push on rollator) was approximately equal for all participants. Mean (SD) for "moderate but comfortable amount of pressure" was $21 \%( \pm 6.57)$ of body weight.

All subjects walked at a comfortable self-selected gait speed as indicated in Table 1. Mean values when walking with rollator and with rollator with push were slightly lower than without rollator (mean difference $-0.02 \mathrm{~m} / \mathrm{s}$ ). Friedmans Anova indicated a significant difference between conditions but post-hoc tests with a Bonferroni Correction significance level of $p<0.001$ showed no statistically significant difference between walking speeds in each condition.

Table 1. Gait Speed (m/s) mean (SD1)

\begin{tabular}{llll}
\hline no rollator2 & rollator3 & rollator + push4 & Friedmans ANOVA5 \\
\hline $1.41(0.15)$ & $1.39(0.15)$ & $1.39(0.14)$ & $p=0.006^{*}$ \\
Post-hoc Wilcoxon Tests6 & & & \\
no rollator $V$ with rollator & rollator V rollator + push & no rollator $V$ with rollator + push & \\
$p=0.19$ & $p=0.50$ & $p=0.01$ & \\
\hline
\end{tabular}

${ }^{1}$ Standard deviation; ${ }^{2}$ baseline, ${ }^{3}$ Rollator walking; ${ }^{4}$ Rollator walking with increased weight- bearing on the handles with a "moderate but comfortable amount of pressure"; ${ }^{5}$ Friedman's ANOVA, * significant values $p<$ $.05 ;{ }^{6}$ Post-hoc tests with Bonferroni Correction significance values $p<0.01$

Table 2. shows the percentage change of maximum EMG activity between walking without rollator (baseline), with rollator and with rollator with a "push" together with effect sizes. Absolute EMG values and statistical analysis for each condition are presented in Table 3.

Table 2. Percentage change of EMG1 values and Effect Sizes between walking without rollator, with rollator \& rollator + push (mean \& SD2)

\begin{tabular}{|c|c|c|c|c|c|c|}
\hline & $\begin{array}{l}\text { Gluteus } \\
\text { maximus }\end{array}$ & $\begin{array}{l}\text { Gluteus } \\
\text { medius }\end{array}$ & $\begin{array}{l}\text { Rectus } \\
\text { femoris }\end{array}$ & $\begin{array}{l}\text { Semi- } \\
\text { tendinosis }\end{array}$ & $\begin{array}{l}\text { Tibialis } \\
\text { anterior }\end{array}$ & $\begin{array}{l}\text { Gastroc- } \\
\text { nemius }\end{array}$ \\
\hline $\begin{array}{l}\% \text { Change of EMG1 } \\
\text { no rollator3 } \mathrm{V} \\
\text { rollator4 }\end{array}$ & $-3.51(18.33)$ & $-13.51(14.91)$ & ) -13.56 (16.84) & $-8.92(20.30)$ & $-10.50(14.63)$ & $-5.97(20.81)$ \\
\hline Effect Size6 & -0.15 & -0.31 & -0.33 & -0.26 & -0.34 & -0.16 \\
\hline $\begin{array}{l}\% \text { Change of EMG1 } \\
\text { no rollator } V \\
\text { rollator + push5 }\end{array}$ & $1-26.73(20.46)$ & $-29.03(19.99)$ & ) -19.15 (26.32) & $-21.01(30.65)$ & $-9.54(21.03)$ & $-10.08(15.41)$ \\
\hline Effect Size6 & $-0.47 \#$ & $-0.45 \#$ & -0.36 & -0.36 & -0.27 & -0.33 \\
\hline
\end{tabular}

${ }^{1}$ Electromyography; ${ }^{2}$ Standard deviation; ${ }^{3}$ baseline, ${ }^{4}$ Rollator walking; ${ }^{5}$ Rollator walking with increased weightbearing on the handles with a "moderate but comfortable amount of pressure"; ${ }^{6} \mathrm{r}=\mathrm{Z} / \mathrm{VN}$; small effect=.10; medium effect= .30; large effect= .50; \# medium to large effect 
Chapter 5

Table 3. Peak of mean EMG1 values over 9 gait cycles for each condition in $\mu \mathrm{V} 2$ (mean \& SD3)

\begin{tabular}{|c|c|c|c|c|c|c|}
\hline & $\begin{array}{l}\text { Gluteus } \\
\text { maximus }\end{array}$ & $\begin{array}{l}\text { Gluteus } \\
\text { medius }\end{array}$ & $\begin{array}{l}\text { Rectus } \\
\text { femoris }\end{array}$ & $\begin{array}{l}\text { Semi- } \\
\text { tendinosis }\end{array}$ & $\begin{array}{l}\text { Tibialis } \\
\text { anterior }\end{array}$ & $\begin{array}{l}\text { Gastroc- } \\
\text { nemius }\end{array}$ \\
\hline No rollator4 & $12.04(6.78)$ & $45.34(27.15)$ & $11.89(5.50)$ & $32.47(27.66)$ & 71.45 (35.29) & 89.93 (231.79) \\
\hline Rollator5 & $11.64(7.38)$ & $40.22(27.97)$ & $10.60(5.77)$ & $29.49(24.65)$ & $62.93(31.65)$ & $50.69(49.48)$ \\
\hline Rollator + push 6 & $8.41(4.96)$ & $31.45(22.46)$ & $9.56(5.23)$ & $25.35(23.26)$ & $63.42(33.95)$ & $77.97(182.76)$ \\
\hline $\begin{array}{l}\text { ANOVA P-value } \\
\text { (CI) } 7\end{array}$ & $<0.001^{* * *}$ & $<0.001^{* * *}$ & $0.006^{*}$ & $0.003^{*}$ & $0.018^{*}$ & 0.080 \\
\hline $\begin{array}{l}\text { Wilcoxon (IQR) } \\
\text { no rollator V } \\
\text { rollator }\end{array}$ & 0.243 & $0.021^{*}$ & $0.013^{*}$ & 0.053 & $0.011^{*}$ & 0.215 \\
\hline $\begin{array}{l}\text { Wilcoxon (IQR) } \\
\text { no rollator V } \\
\text { rollator + push }\end{array}$ & $<0.001 * * *$ & $0.001^{*}$ & $0.006^{*}$ & $0.007^{*}$ & $0.044^{*}$ & $0.013^{*}$ \\
\hline
\end{tabular}

1Electromyography; 2microvolts; 3Standard deviation; 4baseline, 5Rollator walking; 6Rollator walking with increased weight- bearing on the handles with a "moderate but comfortable amount of pressure" 7 Friedman's ANOVA, * significant values $p<.05 ; * * *$ highly significant values $p \leq .001$

For the primary outcome, rollator walking with a "push", a statistically significant reduction of EMG activity in all muscle groups compared to baseline walking without rollator was shown and effect sizes for percentage reductions were medium to large.

Walking with a rollator without a "push" also reduced muscle activity in all muscle groups but changes were statistically significant only in the gluteus medius, rectus femoris, semi-tendinosis and tibialis anterior and effect sizes for percentage reductions were small to medium.

Figure 3. shows a typical example of the effect of walking without rollator, with rollator and with rollator with a "push" on the EMG muscle activity of gluteus medius averaged over nine gait cycles. 


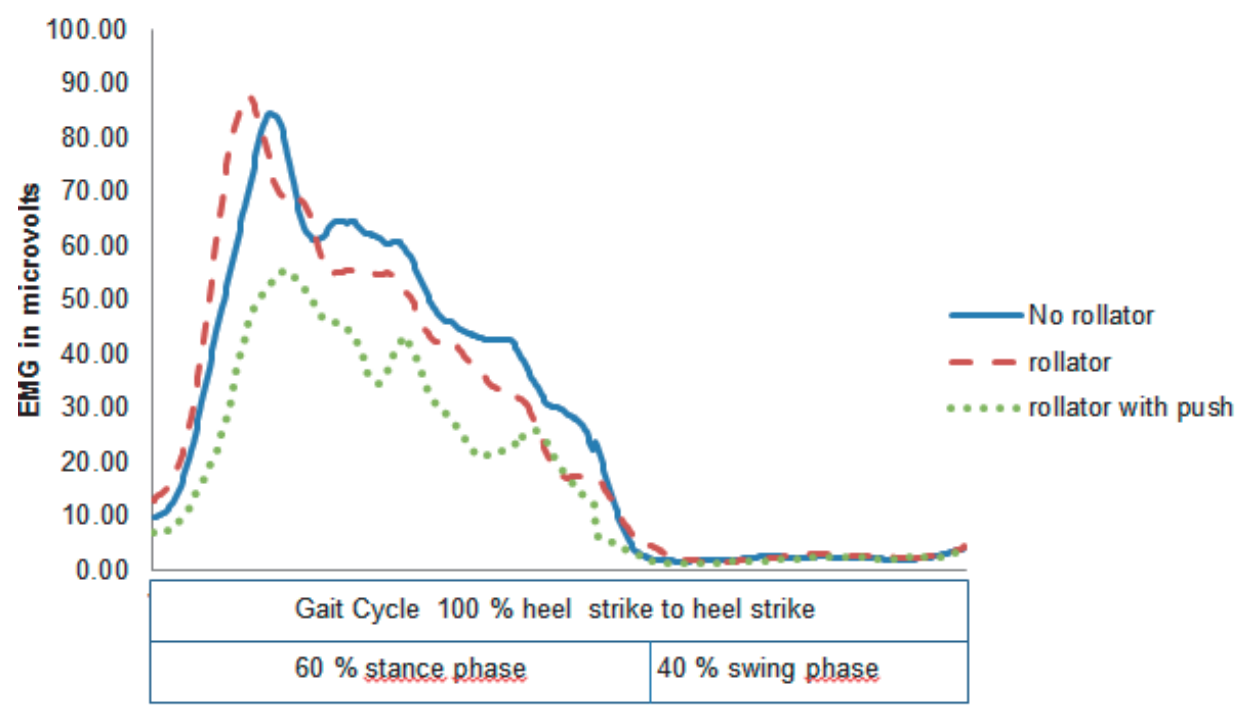

Figure 3. Mean EMG Gluteus Medius activity over 9 Gait Cycles.

In Table 4 . values of trunk sway in degrees in the frontal and sagittal planes when walking without rollator, with rollator and with rollator with a "push" are presented. No statistically significant differences in trunk sway between conditions were found.

Table 4. Trunk sway in frontal (roll) and sagittal (pitch) planes in degrees

\begin{tabular}{lll}
\hline & RoAngRange1 & PiAngRange2 \\
\hline Baseline & $5,84(1,74)$ & $6,59(2,58)$ \\
Rollator & $5,46(1,79)$ & $5,77(1,70)$ \\
Rollator+ & $5,67(1,88)$ & $6,93(1,80)$ \\
Friedman's ANOVA & & 0.080 \\
P-value & 0.196 & \\
\hline
\end{tabular}

1Roll Angular Range, 2Pitch Angular Range

Figure 4. shows a typical example of peak-to-peak angular displacement of the centre of mass in the frontal-plane(roll) and sagittal-plane(pitch) in degrees during each condition in one subject. 
Chapter 5

4a) Walking without rollator
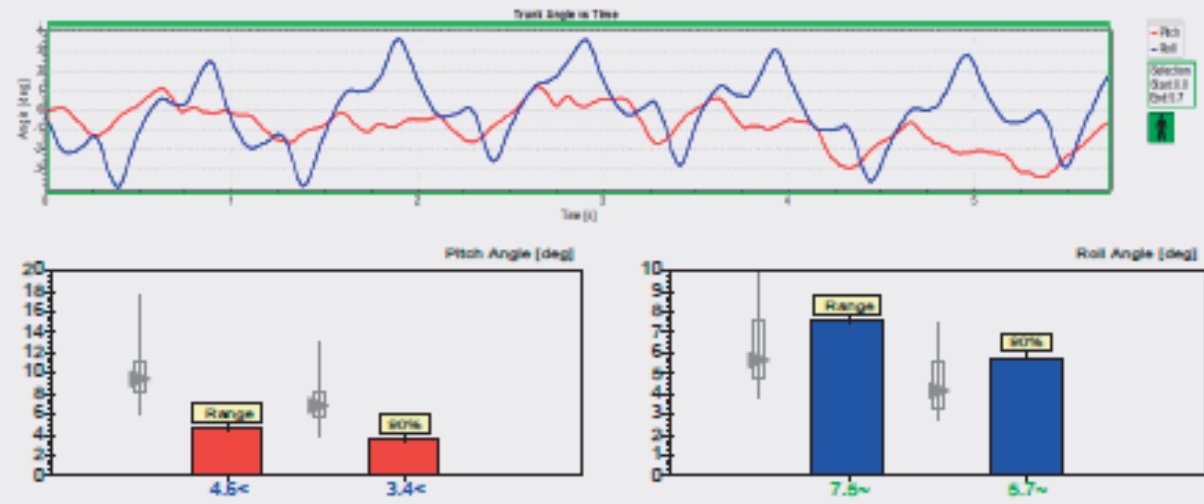

4b) Walking with rollator
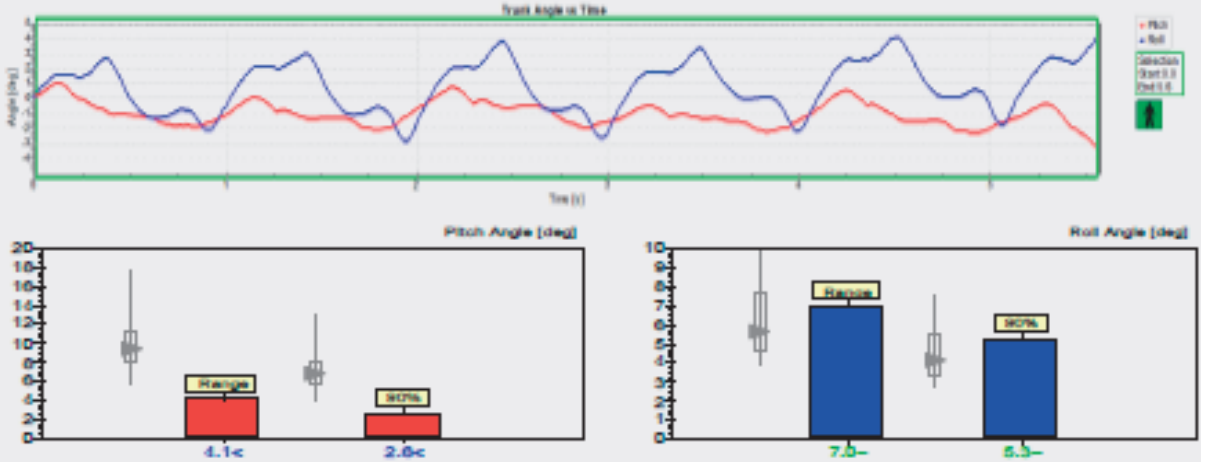

4c) Walking with rollator with additional (moderate) pressure
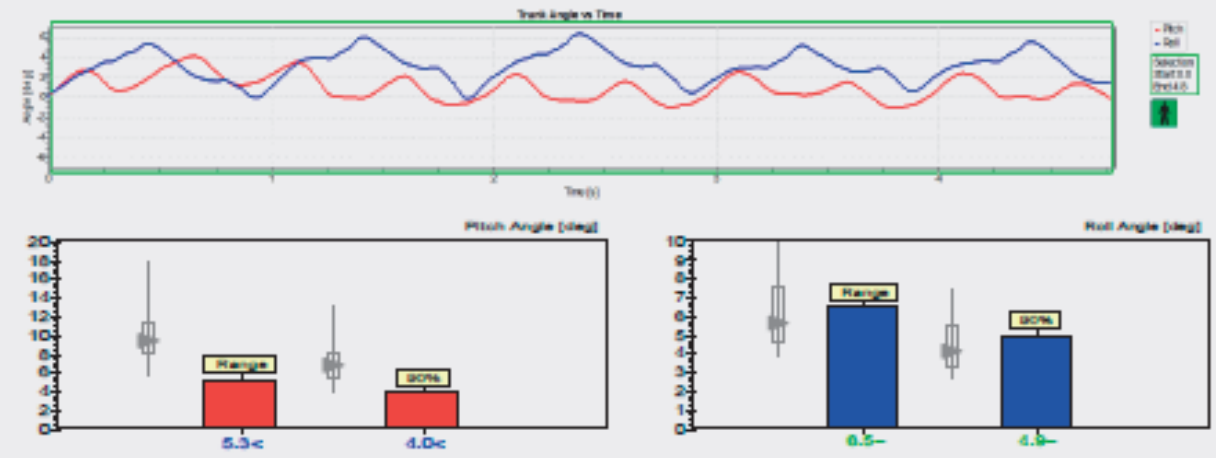

Figure 4. Peak to peak angular displacement of the centre of mass in the frontal (roll) and sagittal (pitch) planes during each condition in one subject. 


\section{DISCUSSION}

This study investigated the influence of walking with a rollator with and without increased weight bearing (push) on muscle activity in the lower extremities and trunk sway in the frontal and sagittal planes compared to walking without a rollator in healthy subjects without gait impairments.

Gait speed reduced minimally when walking with a rollator compared to walking without, but no statistically significant differences were measured. These results confirm the findings of Tung JY et al. 2014 who measured gait speed with and without rollators in healthy young and balance impaired elderly subjects. Further, this degree of change in mean gait speed between conditions, $-0.02 \mathrm{~m} / \mathrm{s}$, has been shown to have minimal influence on EMG activity during walking (Murley GS et al., 2014, Hof AL et al., 2002).

Our results showed that rollator walking consistently reduced EMG muscle activity in all lower extremity muscle groups and that increased weight bearing lead to increased reduction in activity. Indeed a "moderate but comfortable amount of pressure" on the rollator leads to statistically significant reductions in activity in all muscle groups with medium to large effect sizes. These results confirm the first and second hypotheses.

These points could be important during the clinical prescription of rollators although the effect on muscle activity in patient groups must be investigated before definite clinical conclusions can be drawn.

Additionally, although EMG activity is reduced in all investigated muscle groups, it is not clear how much reduction of muscle force this represents. The long-term influence of rollator use on muscle mass and strength needs further research. Vogt et al. (2010) did assess the effect on functional rehabilitation in geriatric patients in comparison to non-rollator users. The study concluded that there was no difference in functional gains between the different groups. However the rollator patients were allowed to use the device during measurement of functional tasks. Schwenk et al. (2011) showed that when assessing mobility using a rollator, initial deficits are underestimated and responsiveness and assessment of change are reduced. This may have been an important confounding factor in these study results.

Trunk sway measurements in our study showed no statistically significant differences in peak to peak angular displacement in the frontal or sagittal planes under all three conditions. Our third hypothesis is therefore also true. This maintenance of stability despite reduced hip muscle activity indicates that compensatory postural strategies were induced by rollator walking. Hip abductor activity is usually responsible for maintaining a level pelvis in the frontal plane and hip extensor muscle activity acts to restrain anterior pelvic tilt during the loading response (Perry, 1992).Therefore other mechanisms than hip muscle activity must have prevented increased pelvic movement and consequently trunk sway during rollator walking. Chee et al. (2013) investigated rollator use in multiple sclerosis patients and concluded that the device would "compensate for 
hip abductor weakness by providing improved frontal plane stability through upper extremity control". Tung JY , Gage WH et al. (2014) investigating rollator use in balance impaired subjects and healthy subjects under challenging balance conditions also concluded that "the upper limbs play a greater roll in controlling frontal plane balance during rollator assisted walking when lower-limb balance capabilities are limited or impaired". A further study with stroke and brain inury patients also concluded that the degree of upper limb use correlated with increasingly difficult walking environments.(Tung JY, Chee et al. 2014)

We postulate that this upper extremity activity is responsible for maintaining trunk stability in both frontal and sagittal planes.

Walking with a rollator therefore reduces muscle activity in the lower limbs but maintains trunk and balance stability by facilitating compensatory activity of the upper extremities. This may represent the inhibition of normal motor patterns for the control of walking and balance.

As the degree of spontaneous lateral trunk sway is an important indicator for fall risk (Maki et al.1997) and correlates with balance stability (Corporaal et al., 2013; Gill et al., 2001; Goutier et al., 2010) assistive devices which influence the control of trunk sway need to be carefully considered when prescribed in clinical practice. If treatment goals are to improve, as far as possible, balance reactions e.g. during fall prevention programs or early post-stroke rehabilitation, rollators may not be optimal. Conversely if the priority of treatment is to restore stability, regardless of the strategy used or simply to unload joints post-operatively then rollators may be the assistive device of choice. Indeed different patient groups show some differences in loading and upper limb activity depending on need (Fast et al., 1995). Axial forces are higher and rhythmical in non-weight bearing post-operative patients, lower and more sporadic in ataxic patients.

However further studies are needed to clarify the short and long-term effects of rollator use in different patient groups. It may be that parameters such gait speed and muscle activation levels and patterns are influenced differently depending on pathology. Long-term use may also effect responses compared to the healthy subjects in this study who practiced for only five minutes before measurements began.

Salminen et al. (2009) stated that 'The effectiveness of an assistive device is the extent to which it produces a beneficial outcome in a routine setting'. We suggest that this definition merely considers the immediate effect of assistive devices. Long-term as well as short term effects need to be clarified.

\section{CONCLUSION}

The results of our study show that rollator-walking reduces lower-limb muscle activity but trunk-sway remains unchanged in healthy subjects. We postulate that stability is gained through forces generated by the upper-limbs, suggesting that rollator use facili- 
tates compensatory postural and balance control strategies. These findings should be investigated in patient groups to allow an evidence based and differentiated prescription of rollators in clinical practice.

\section{CONFLICT OF INTEREST}

None.

\section{ACKNOWLEDGEMENTS}

We would like to thank the Bildungszentrum Gesundheit Basel-Stadt for all their support during the research. 


\section{REFERENCES}

Field A, 2005 Discovering Statistics Using SPSS., 2nd ed. Sage, London.

Alkjaer T, Larsen PK, Pedersen G, Nielsen LH, Simonsen EB 2006 Biomechanical analysis of rollator walking. Biomed Eng Online 5, 2.

Allum JH, Carpenter MG 2005 A speedy solution for balance and gait analysis: angular velocity measured at the centre of body mass. Curr Opin Neurol 18, 15-21.

Allum JH, Carpenter MG, Adkin AL 2001 Balance control analysis as a method for screening and identifying balance deficits. Ann N Y Acad Sci 942, 413-427.

Bateni H, Maki BE 2005 Assistive devices for balance and mobility: benefits, demands, and adverse consequences. Arch Phys Med Rehabil 86, 134-145.

Bischoff-Ferrari HA, Conzelmann M, Stähelin HB, Dick W, Carpenter MG, Adkin AL, Theiler R, Pfeifer M, Allum $\mathrm{JH} 2006$ Is fall prevention by vitamin D mediated by a change in postural or dynamic balance? Osteoporos Int 17, 656-663.

Braun T, Marks D, Zutter D, Grüneberg C 2014 The impact of rollator loading on gait and fall risk in neurorehabilitation - a pilot study. Disabil Rehabil Assist Technol 17, 1-7.Chee JN, Gage WH, Mcllroy WE, Zabjek KF 2013 Foot placement patterns of female rollator users with multiple sclerosis in the community. Disabil Rehabil 35, 27-35.

Corporaal SH, Gensicke H, Kuhle J, Kappos L, Allum JH, Yaldizli Ö 2013 Balance control in multiple sclerosis: correlations of trunk sway during stance and gait tests with disease severity. Gait Posture 37, 55-60.

Eggermont LH, van Heuvelen MJ, van Keeken BL, Hollander AP, Scherder EJ 2006 Walking with a rollator and the level of physical intensity in adults 75 years of age or older. Arch Phys Med Rehabil 87, 733-736.

Fast A, Wang FS, Adrezin RS, Cordaro MA, Ramis J, Sosner J 1995 The Instrumented Walker: Usage Patterns and Forces. Arch Phys Med Rehabil 76, 484-91

Gill J, Allum JH, Carpenter MG, Held-Ziolkowska M, Adkin AL, Honegger F, Pierchala K 2001 Trunk sway measures of postural stability during clinical balance tests: effects of age. J Gerontol A Biol Sci Med Sci 56, M438-447.

Hof AL, Elzinga H, Grimmus W, Halbertsma JPK 2002 Speed dependence of averaged EMG profiles in walking, Gait Posture 16, 78-86.

Goutier KM, Jansen SL, Horlings CG, Küng UM, Allum JH 2010 The influence of walking speed and gender on trunk sway for the healthy young and older adults. Age Ageing 39, 647-650.

Gribble PA, Hertel J 2004 Effect of hip and ankle muscle fatigue on unipedal postural control. J Electromyogr Kinesiol 14, 641-646.

Gupta RB, Brooks D, Lacasse Y, Goldstein RS 2006 Effect of rollator use on health-related quality of life in individuals with COPD. Chest 130, 1089-1095.

Hermens HJ, Freriks B, Disselhorst-Klug C, Rau G 2000 Development of recommendations for SEMG sensors and sensor placement procedures. J Electromyogr Kinesiol 10, 361-374.

Horlings CG, Küng UM, van Engelen BG, Voermans NC, Hengstman GJ, van der Kooi AJ, Bloem BR, Allum JH 2009 Balance control in patients with distal versus proximal muscle weakness. Neuroscience 164, 1876 1886.

Ishikura T 2001 Biomechanical analysis of weight bearing force and muscle activation levels in the lower extremities during gait with a walker. Acta Med Okayama 55, 73-82.

Maguire C, Sieben JM, Frank M, Romkes J 2010 Hip abductor control in walking following stroke -- the immediate effect of canes, taping and TheraTogs on gait. Clin Rehabil 24, 37-45.

Maki BE, Holliday PJ, Topper AK 1994 A prospective study of postural balance and falling in an ambulatory and independent elderly population. J.Gerontol 49 (2), 72-84 Maki BE, Mcllroy WE 1997 The role of limb movements in maintaining upright stance: the "change-in-support" strategy. Phys Ther 77, 488-507.

Malviya A, Richards J, Jones RK, Udwadia A, Doyle J 2005 Reproducibilty of partial weight bearing. Injury 36, 556-559. 
Murley GS, Menz HB, Landorf KB 2014 Electromyographic patterns of tibialis posterior and related muscles when walking at different speeds. Gait Posture 39, 1080-1085.

Negahban H, Etemadi M, Naghibi S, Emrani A, Shaterzadeh Yazdi MJ, Salehi R, Moradi Bousari A 2013 The effects of muscle fatigue on dynamic standing balance in people with and without patellofemoral pain syndrome. Gait Posture 37, 336-339.

Neumann DA 1998 Hip abductor muscle activity as subjects with hip prostheses walk with different methods of using a cane. Phys Ther 78, 490-501.

Pai YC, Patton J 1997 Center of mass velocity-position predictions for balance control. J Biomech 30, 347-354.

Pardo RD, Deathe AB, Winter DA 1993 Walker user risk index. A method for quantifying stability in walker users. Am J Phys Med Rehabil 72, 301-305.

Perry J 1992 Gait Analysis: Normal and Pathological Function. SLACK.

Runge CF, Shupert CL, Horak FB, Zajac FE 1999 Ankle and hip postural strategies defined by joint torques. Gait Posture 10, 161-170.

Salminen AL, Brandt A, Samuelsson K, Toytari O, Malmivaara A 2009 Mobility devices to promote activity and participation: a systematic review. J Rehabil Med 41, 697-706.

Schwenk M, Schmidt M, Pfisterer M, Oster P, Hauer K 2011 Rollator use adversely impacts on assessment of gait and mobility during geriatric rehabilitation. J Rehabil Med 43, 424-429.

Smith TO, McCabe C, Lister S, Christie SP, Cross J 2012 Rehabilitation implications during the development of the Norwich Enhanced Recovery Programme (NERP) for patients following total knee and total hip arthroplasty. Orthop Traumatol Surg Res 98, 499-505.

Tung JY, Chee JN, Zabjek KF, Mcllroy WE 2014 Combinibng amulatory and laboratory assessment of rollator use for balance and mobility in neurologic rehabilitation in-patients. Disbil Rehabil Technol Early Online 1-8.

Tung JY, Gage WH, Poupart P, Mcllroy WE 2014 Upper limb contributions to frontal plane balance control in rollator-assisted walking. Assist Technol 26, 15-21; quiz 22-13.

Vogt L, Lucki K, Bach M, Banzer W 2010 Rollator use and functional outcome of geriatric rehabilitation. J Rehabil Res Dev 47, 151-156.

Winter DA, Prince F, Frank JS, Powell C, Zabjek KF 1996 Unified theory regarding A/P and M/L balance in quiet stance. J Neurophysiol 75, 2334-2343.

Youdas JW, Kotajarvi BJ, Padgett DJ, Kaufman KR 2005 Partial weight-bearing gait using conventional assistive devices. Arch Phys Med Rehabil 86, 394-398. 



\section{Chapter}

\section{The effect of crutches, an orthosis TheraTogs and no walking aids on the recovery of gait in a patient with delayed healing post hip fracture: a case report.}

Authors

Clare Maguire, Judith M Sieben, Heike Scheidhauer, Jacqueline Romkes, Zorica Suica, Robert de Bie.

Physiotherapy Theory and Practice, 32:1, 69-81 


\section{ABSTRACT}

Accelerated rehabilitation following hip fracture and joint-replacement, including early unrestricted weight-bearing and muscle strengthening has gained importance in hastening functional recovery and hospital discharge. The influence of walking-aids on these parameters is sparsely investigated. In this single-subject case report we document the effect of walking with crutches, an orthotic garment and strapping-system, TheraTogs, and no walking-aids over three four week periods on walking speed, trunksway and muscle activity measured with electromyography(EMG). The patient was a 49 year old female showing delayed healing following a conservatively treated avulsion fracture of the greater trochanter twelve weeks previously with a fourteen year history of total hip arthroplasty. EMG-analysis showed muscle activity increased with TheraTogs and decreased with crutches compared to walking with no-aids. Walking speed improved at a faster rate in the TheraTogs-phase than crutches-phase and reduced in no walking-aids phase. Mean speed(SD)m/s for each phase was: crutches 1.11(0.08), TheraTogs 1.35(0.11), no-aids 1.19(0.14). Trunk-sway increased in the crutch and noaids phases, and became more stable in TheraTogs-phase. In this patient, function and recovery rate of all measured parameters increased more in the TheraTogs-phase than the crutches or no-aids phase. This may be because muscle activity was facilitated enabling active support of recovering structures.

\section{KEYWORDS}

Accelerated rehabilitation; hip fracture; muscle strength; unrestricted weight-bearing; walking aids 


\section{INTRODUCTION}

Accelerated rehabilitation for patients following hip fracture or total hip replacement (THR) has become established over the last decade to hasten functional recovery and shorten hospital stay. (Hol, van Grinsven, Lucas, van Susante, \& van Loon, 2010; Hung, Egol, Zuckerman, \& Siu, 2012; Robbins et al., 2014; Tayrose et al., 2013) Early or immediate unrestricted weight-bearing is an important aspect of these programmes.

Additionally, the importance of regaining hip muscle strength to improve patient outcomes and reduce health care costs for patients following joint replacement and hip fracture patients has been demonstrated. (Piva et al., 2011) (Borrelli, Ricci, Anglen, Gregush, \& Engsberg, 2006; Zhao, Chen, Lee, \& Wu, 2013)

Deficiency of the hip abductor mechanism is however a common complication following THR with a reported incidence of up to 20\%.(Garcia, Picado, \& NogueiraBarbosa, 2010; Masonis \& Bourne, 2002) This may be pre-existing and found during surgery or it may occur during surgery as a result of damage to the superior gluteal nerve or due to the detachment of the abductor muscles from the greater trochanter. (Odak \& Ivory, 2013) Fracture of the femur is also a common complication with five percent involving the greater trochanter. (Pritchett, 2001) Recovery may occur spontaneously or surgery may be necessary if conservative treatment fails. For these patients unrestricted weight bearing and muscle strengthening can be delayed.

As the choice of walking aid in orthopaedic rehabilitation clearly influences weight bearing status and has a significant impact on muscle activity (Maguire, Sieben, Frank, \& Romkes, 2010; Neumann, 1998; Sonntag, Uhlenbrock, Bardeleben, Kading, \& Hesse, 2000) the prescription of appropriate assistive devices to optimise recovery is important.

Canes and crutches enable partial and non-weight bearing gait thus unloading joints and potentially reducing pain when walking. However they have also been shown, in cross-sectional studies, to reduce muscle activity, gait speed and cadence. (Maguire et al., 2010; Neumann, 1998; Sonntag et al., 2000) Crutches cause abnormal activation patterns in hip abductor muscles following hip arthroplasty(Sonntag et al., 2000) and restricted weight bearing through assistive devices which use the upper limbs significantly increases energy expenditure. (Westerman, Hull, Hendry, \& Cooper, 2008)

As many patients are unable to walk without some form of walking aid immediately post-operatively, assistive devices which support the affected structures without reducing muscle activity or requiring the use of the upper limbs could be more beneficial than hand-held walking aids. This may be particularly relevant for patients with a defective hip abductor mechanism.

An elasticized orthotic garment and strapping system named TheraTogs ${ }^{\mathrm{TM}}$ (Telluride, Colorado) which does not require use of the arms, has shown promising results regarding muscle activity when tested with patients following stroke. (Maguire et al., 2010) This study showed that hip abductor activity increased by $16.5 \%$ when walking with the orthotic-garment compared to walking without walking aids. Studies have also 
indicated that TheraTogs facilitates improvements in bony-alignment and kinematic gait parameters in children with neurological conditions (Abd El-Kafy, 2014; Richards, Morcos, Rethlefsen, \& Ryan, 2012). To our knowledge this system has not been tested in orthopaedic patients.

In this clinically driven single case report we outline the effects of crutches, the orthosis TheraTogs and no walking aids during the gait rehabilitation of a 49 year old patient with delayed healing of a conservatively treated avulsion fracture of the greater trochanter having undergone total hip replacement 14 years previously. Written consent was obtained from the patient.

\section{Case Description}

The patient was a 49 year old female $(1 \mathrm{~m} 60 \mathrm{~cm}, 50 \mathrm{~kg})$ who had undergone a left total hip arthroplasty 14 years previously, suffering an incomplete lesion of the lumbar plexus during surgery resulting in residual muscle weakness. Subsequent replacement of the acetabulum was necessary twice, seven then eight years after original surgery due to initial incorrect positioning of the acetabulum component. Twelve weeks before the reported course of treatment the patient had stumbled causing an avulsion fracture of the greater trochanter, leaving the prosthetic implant at this level without bony covering and having direct contact with overlying soft tissues (Figure 1.). Surgical repair was not recommended at this stage as the original incorrect positioning of the acetabulum and repeated surgical corrections had resulted in poor bone quality. The fracture was treated conservatively and the patient was non-weight bearing for five weeks after which she could progress to partial then to full weight bearing as pain allowed.

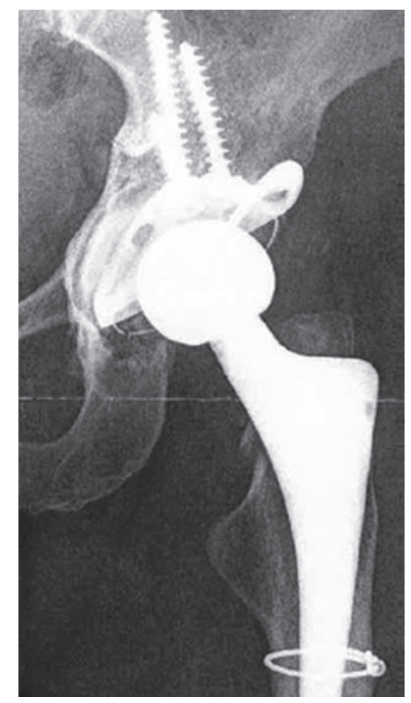

Figure 1. Plain radiograph (AP-view) taken on initial presentation, showing an avulsion fracture of the greater trochanter, leaving the shoulder of the prosthesis in direct contact with soft tissues (circled). 
Twelve weeks post-fracture the patient was continuing to walk with elbow crutches and attempting to reduce crutch use. Normal progression to full weight- bearing without assistive devices was delayed due to high levels of pain. Physiotherapy treatment had consisted of advice and exercise prescription when in hospital and nothing further since discharge. The patient had now requested assistance with the reduction of crutch use and the progression to full weight-bearing.

Before fracture the patient walked independently without aids for 2-3 km, at which point she would rest. Stair climbing was possible using a bannister. A slight Trendelenburg was visible after walking long distances, indicating underlying hip abductor weakness.

The patient had no other medical or surgical complaints.

\section{Clinical Examination}

\section{Social History}

The patient worked as a teacher. She had pain standing for long periods in the classroom and was currently working more with office and administrative tasks. She was able to drive to and from work. She lived with her husband in a house with stairs. Active hobbies were cycling (usually tandem), E-biking (a bicycle with an integrated electric motor) and diving.

\section{Pain}

A deep burning pain becoming sharp and intense began over the lateral aspect of the left hip (site of greater trochanter fracture) (P1 on body chart Figure 2a) after 15-20 minutes walking on flat ground and partial weight bearing with two crutches. This radiated to the medial aspect of the knee joint if the patient continued to walk (P2). These pains varied between 3-8 on the Visual Analogue Scale (VAS). Pain began at 3 and became quickly worse if the patient attempted to walk through the pain or to reduce weight bearing on the crutches. Pain was also present in the left buttock when attempting to reduce weight-bearing on the crutches (P3) (VAS 4-5) (Figure 2b). This increased significantly during stair climbing or after sitting for 30 to 60 minutes. Generalised groin pain (P4) (VAS 3-4) was not present in the morning but increased throughout the day. All pain was reduced after 1-3 hours of complete rest in lying with the hip in extension and slight abduction. 


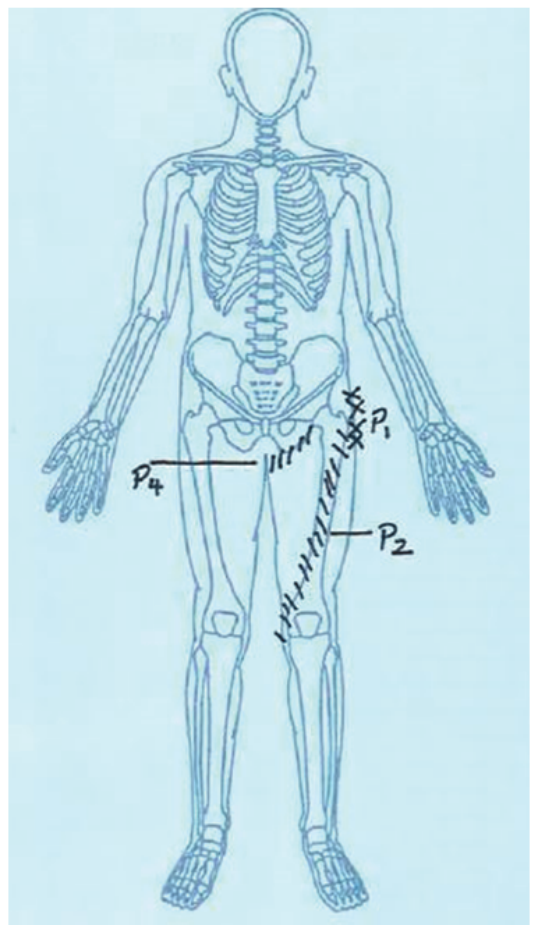

Figure 2a. Body chart showing areas of pain P1, P2 and P4.

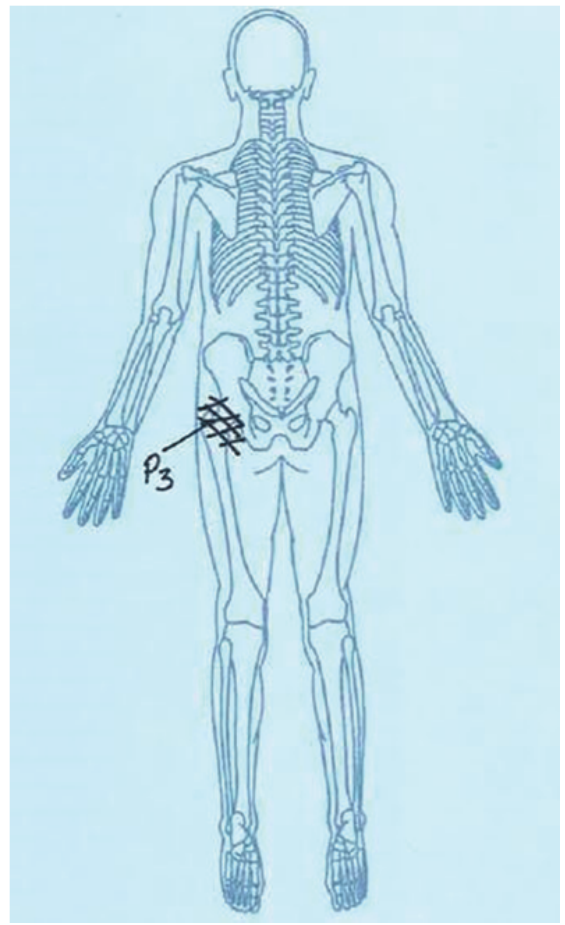

Figure $2 \mathrm{~b}$. Body chart showing areas of pain P3.

\section{Medication}

Paracetamol 325mg / Tramadol 37.5 mg taken 2-4 times daily as needed for pain.

\section{Observation}

\section{Gait}

The patient had a Trendelenberg gait pattern when walking without crutches.

\section{Standing}

Weight bearing was predominantly on the right leg. Atrophy of left gluteus medius and hypertrophy of left tensor fascia lata. Atrophy of left-sided quadriceps, hamstrings, and gastrocnemius (Table 1).

Palpation Generalised tenderness over the lateral aspect of the left hip. Over-activity and hypertrophy of left tensor fascia lata.

Reflexes Patellar Tendon Reflex Reduced on the left. Normal on the right. Achilles tendon reflex - absent on the left. Normal on the right. 
Table 1 Active and passive Range of Movement in the hip, knee and ankle joints of the left leg. Muscle strength of hip and lower limb muscle groups tested with the Manual Muscle Test

\begin{tabular}{|c|c|c|c|c|c|c|}
\hline & \multicolumn{6}{|c|}{ Hip joint Range of Movement (ROM) and Muscle Strength } \\
\hline & Flexion & Extension & Abduction & Adduction & $\begin{array}{l}\text { Internal } \\
\text { Rotation }\end{array}$ & $\begin{array}{l}\text { External } \\
\text { Rotation }\end{array}$ \\
\hline Active ROM & $80^{\circ}$ & $0^{\circ}$ & $30^{\circ}$ & $0^{\circ}$ & $5^{\circ}$ & $20^{\circ}$ \\
\hline Left & limited by pain & limited by pain & limited by pain & limited by pain & limited by pain & limited by pain \\
\hline Right & Full range & Full range & Full range & Full range & Full range & Full range \\
\hline Passive ROM & $100^{\circ}$ & $0^{\circ}$ & $40^{\circ}$ & $10^{\circ}$ & $10^{\circ}$ & $30^{\circ}$ \\
\hline Left & limited by pain & limited by pain & limited by pain & limited by pain & limited by pain & limited by pain \\
\hline Right & Full range & Full range & Full range & Full range & Full range & Full range \\
\hline $\begin{array}{l}\text { Strength: Manual } \\
\text { Muscle Test Left }\end{array}$ & 4 & 4 & $2-3$ & 4 & 3 & 4 \\
\hline \multirow[t]{3}{*}{ Right } & 5 & 5 & 5 & 5 & 5 & 5 \\
\hline & \multicolumn{6}{|c|}{ Range of Movement and Strength in Lower Limbs } \\
\hline & Knee flexion & Knee extension & $\begin{array}{l}\text { Ankle } \\
\text { dorsiflexion }\end{array}$ & $\begin{array}{l}\text { Ankle } \\
\text { plantarflexion }\end{array}$ & Ankle inversion & Ankle eversion \\
\hline $\begin{array}{l}\text { Active ROM } \\
\text { Left }\end{array}$ & Full range & Full range & $80^{\circ}$ & Full range & Full range & Full range \\
\hline Right & Full range & Full range & Full range & Full range & Full range & Full range \\
\hline $\begin{array}{l}\text { Passive ROM } \\
\text { Left }\end{array}$ & Full range & Full range & Full range & Full range & Full range & Full range \\
\hline Right & Full range & Full range & Full range & Full range & Full range & Full range \\
\hline $\begin{array}{l}\text { Strength: Manual } \\
\text { Muscle Test Left }\end{array}$ & 4 & 4 & 3 & 4 & 4 & 3 \\
\hline Right & 5 & 5 & 5 & 5 & 5 & 5 \\
\hline
\end{tabular}

Table 2. Problem list based on the International Classification of Functioning (ICF)

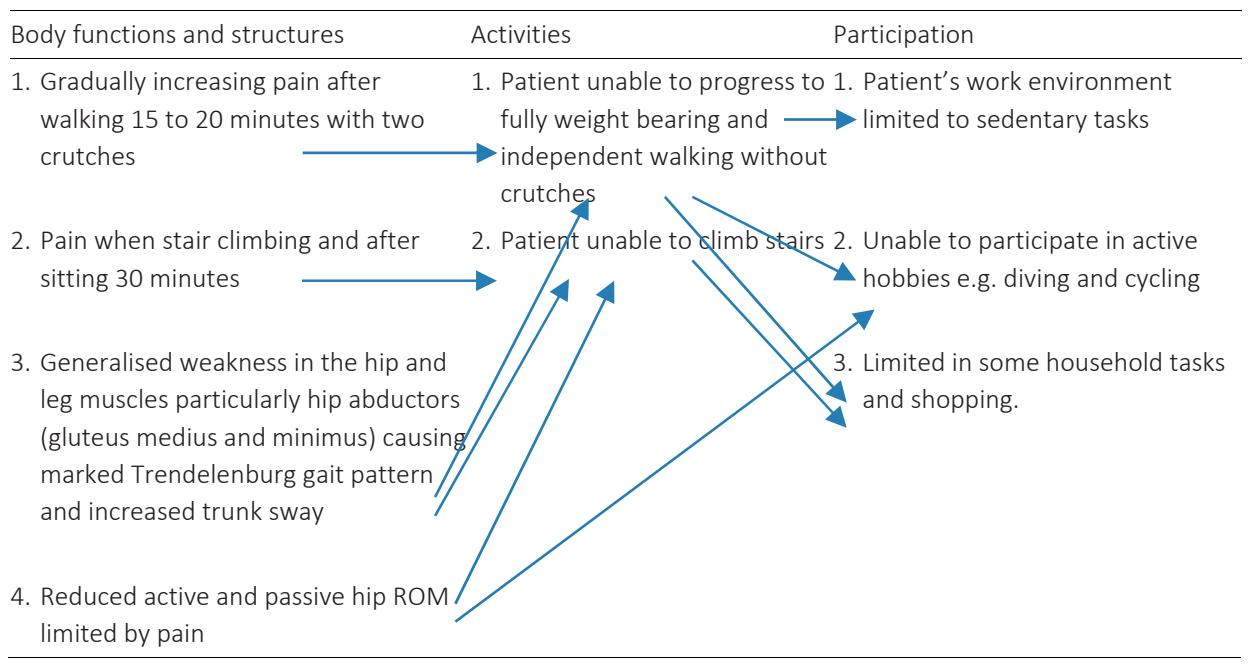




\section{Problem Analysis and Clinical Hypotheses}

The dislocated greater trochanter (displaced dorsally and cranially as seen on x-ray) left the hip prosthesis without bony covering. This caused the shoulder of the prosthesis to irritate overlying soft tissues during all hip movements but particularly during joint loading, causing increased inflammation and pain. The patient was able to walk short distances with crutches as the joint was partially unloaded. Walking without aids increased loading and therefore soft tissue irritation and pain (Table 2).

As the greater trochanter is the insertion for gluteus medius and gluteus minimus, the displacement of this fragment also led to a defective hip abductor mechanism leading to compensation and increased activity of tensor fascia lata (TFL).

Weakness in the other muscle groups was a long-standing problem caused by the incomplete lesion of the lumbar plexus during surgery 14 years previously. This was also the cause of the reduced spinal reflexes.

No further deficits in the lumbar region which may have affected gait function (e.g. core stability, mobility) were found.

\section{Physiotherapy Diagnosis and Treatment Plan}

As pain was caused by the ongoing irritation and inflammation of tissues overlying the exposed prosthesis when walking, it was considered that short term, symptomatic treatment of pain with physiotherapy interventions would not be effective. In order to enable pain free or pain reduced walking without aids, treatment should attempt to increase support around the hip joint and exposed prosthesis during weight bearing. This could be achieved primarily through strengthening of all hip muscle groups including the remaining hip abductor musculature (TFL and possibly muscular contractile elements from gluteus maximus which can fuse with the gluteal aponeurosis)(Netter, $\mathrm{H}$, \& Frank, 2014). Crutch use should be gradually reduced as muscle strength increases. A muscle strengthening program combined with a gradual reduction of crutch use was prescribed.

\section{Prognostic Indicators}

Compensatory strategies are developed by a large proportion of patients in the presence of defective hip abductor mechanisms.(Howell, Biggs, \& Bourne, 2001; Odak \& Ivory, 2013; Williams \& Cohen, 2009) The physical requirements necessary to develop these strategies have not been reported (as far as the authors are aware). Positive predictive indicators in this patient which may have supported this ability were considered to be age (49), low BMI, moderate levels of cardiovascular fitness and high motivation. However it was clear that the challenges posed by the exposed prosthesis could not be reduced with conservative treatment. Significant increases in muscle strength in sur- 
rounding muscle groups would be necessary to adequately support the hip joint and exposed soft tissues. The large changes required in muscle strength to enable independent gait and the incomplete lesion of the lumbar plexus were considered negative prognostic indicators.

As the patient was very eager to attempt to achieve her goals without surgery, and because surgery was not recommended at this stage due to weakening of the bone from previous operations, conservative management as outlined was indicated (Table 3).

Table 3. Aims of treatment related to ICF problem list (formulated with patient)

\begin{tabular}{|c|c|c|}
\hline $\begin{array}{l}\text { Short term treatment aims } \\
\text { (2-6 weeks) }\end{array}$ & $\begin{array}{l}\text { Long term treatment aims } \\
\text { (6-12 weeks) }\end{array}$ & $\begin{array}{l}\text { Long term treatment aims } \\
\text { (6-12 weeks) }\end{array}$ \\
\hline Body functions and structures & Activities & Participation \\
\hline $\begin{array}{l}\text { 1. Strengthening of hip and lower } \\
\text { extremity muscle groups } \\
\text { particularly hip abductor and } \\
\text { extensor groups }\end{array}$ & $\begin{array}{l}\text { 1. Improved gait pattern with } \\
\text { reduced Trendelenburg and trunk } \\
\text { sway }\end{array}$ & $\begin{array}{l}\text { 1. Patient able to stand, walk and } \\
\text { climb stairs as necessary at work } \\
\text { and during household tasks } \\
\text { without walking aids }\end{array}$ \\
\hline $\begin{array}{l}\text { 2. Graded and supervised reduction } \\
\text { of crutch walking (increasing } 10 \\
\text { minutes pain free per week) }\end{array}$ & $\begin{array}{l}\text { 2. Pain free, independent walking } \\
\text { without walking aids for } 2-3 \mathrm{~km} \\
\text { (approx. } 1 \text { hour) }\end{array}$ & $\begin{array}{l}\text { 2. Able to participate in more active } \\
\text { hobbies e.g. cycling }\end{array}$ \\
\hline $\begin{array}{l}\text { 3. Increased active and passive hip } \\
\text { ROM }\end{array}$ & $\begin{array}{l}\text { 3. Ability to climb stairs using a } \\
\text { bannister pain free }\end{array}$ & \\
\hline
\end{tabular}

\section{Outcome Measures}

Outcome measurements consisted of: 1) Gait speed measured twice weekly. Changes in gait speed in a female population following hip fracture have been shown to indicate meaningful functional change. (Alley et al., 2011); 2) Dynamic hip muscle function measured twice weekly evaluated through trunk sway using the SwayStar instrumentation (see below). This method was chosen because direct measurement of muscle strength using the manual muscle test was painful for hip abductor muscles. Trunk sway has been used in hip fracture and normal subjects to assess abductor muscle function.(Archdeacon et al., 2008; Klemetti, Steele, Moilanen, Avela, \& Timonen, 2014); and 3) Distance and time walked without crutches with minimal or no pain.

To measure gait speed and trunk sway the patient was instructed to stand with feet level at the starting line of an $8 \mathrm{~m}$ marked level track and when given the cue to start, to walk at a comfortable speed and to stop at the end. Time to complete was measured using the SwayStar instrumentation (see below). Gait speed was calculated.

The SwayStar balance system (Balance International Innovations $\mathrm{GmbH}$, Iseltwald, Switzerland), consists of two angular velocity sensors (fibre-optic gyroscopes). SwayStar was attached via a belt to the patient at the level L2/3 in the midline. The sensors were attached to a computer via a Bluetooth communication which sampled the velocity signals every $100 \mathrm{~ms}$ and numerically integrated the velocity signals to yield angular 
displacement. Trunk sway was measured as the Total Angle Area (TAA) displacement in degrees $^{2}$ of the centre of mass (level L2/3) during $8 \mathrm{~m}$ walk. This was calculated from total movement in the frontal and sagittal planes. The SwayStar has been used to assess trunk sway in many pathologies and differing age groups. (Allum \& Carpenter, 2005; Bischoff-Ferrari et al., 2006; Corporaal et al., 2013; Goutier, Jansen, Horlings, Küng, \& Allum, 2010; Horlings et al., 2009)

The measurement results are presented in Table 5. and plotted in Figures 6. and 7. to show changes over time during the different rehabilitation phases. "Celeration" or "Split Middle" lines, developed to quantify rate of change over time for single case reports or studies, have been plotted to show change in each rehabilitation phase.

\section{Muscle Activity Walking with and without Assistive Devices.}

Muscle activity when walking without walking aids, with TheraTogs and with crutches using electromyography (EMG) was measured. Measurements took place on different days. Walking without aids and with crutches was compared first, followed by walking without aids and with TheraTogs.

\section{Surface EMG Measurements.}

Surface electromyography (EMG) was used to measure muscle activity with a 16 bipolar channel Porti system (TMS International, Enschede, the Netherlands) of the gluteus maximus and gluteus medius (standard SENIAM electrode placement (Hermens, Freriks, Disselhorst-Klug, \& Rau, 2000) despite avulsion fracture to investigate muscle activity at this level and possible compensation mechanisms), tensor fascia lata, quadriceps and hamstrings of the left (affected) leg when walking with and without aids. Gait cycles were defined as heel strike to heel strike of the same leg determined by a foot switch placed mid-heel and synchronised with the Porti-system.

EMG activity during maximum voluntary contraction (MVC) for each muscle was collected on each occasion using the protocol described by Konrad (Konrad, 2006)

\section{EMG Data Collection}

Data was collected for each muscle over nine gait cycles as the patient walked at a selfselected, comfortable speed. Skin was prepared for surface EMG placement, firstly shaved over the relevant muscles and cleaned with alcohol. Noraxon bipolar $\mathrm{Ag} / \mathrm{AgCl}$ surface EMG electrode pairs with an electrode diameter of $10 \mathrm{~mm}$ and an interelectrode spacing of $20 \mathrm{~mm}$ (Noraxon USC, Inc.) were placed onto the skin overlying these muscles following the "European Recommendations for Surface Electromyography" (SENIAM) guidelines.(Hermens et al., 2000) The ground electrode was placed overlying the sacrum. 
EMG was measured at a sampling rate of $2048 \mathrm{~Hz}$ without filtering, so that the signals were measured including Direct Current (DC). The digitalised data was high pass filtered with a fourth order filter with a cut off frequency of $10 \mathrm{~Hz}$. The EMG signals were full wave rectified and each data-set of one gait cycle was interpolated to 500 datapoints using the MATLAB software package(CThe MathWorks, Inc, 1994-2014). The data was exported to Microsoft Excel ${ }^{\circledR}$ (Vers. 14.1.0, 2011) for further analysis.

\section{EMG Analysis}

Mean EMG muscle activity for each muscle in each gait cycle was measured and a maximum of mean values and the standard deviation over nine gait cycles were calculated. EMG activity during maximum voluntary contraction (MVC) for each muscle (remeasured during each measurement at the beginning and end of each phase) was then determined. Maximum of the mean EMG activity during walking as a percentage of MVC was calculated when walking without aids, with TheraTogs and with crutches. Percentage changes between conditions were calculated. Measurement results are presented in Table 4 and Figure 4.

\section{Intervention}

\section{Crutch Walking (Weeks 1-4)}

The patient continued working in a sedentary job and was able to drive a car. Hydrotherapy was completed twice weekly for active left hip and leg strengthening and mobilising exercises within pain limits. A home exercise programme of strengthening and stretching exercises was also completed within pain limits daily. The patient also walked $500 \mathrm{~m}-1 \mathrm{~km}$ each day and had a structured programme to reduce crutch use. Initially two crutches were used for the whole $1 \mathrm{~km}$ distance. On the basis of the structured programme crutch use was reduced.

\section{Response to Treatment}

After two weeks the patient could walk 500m (7-12 minutes) without crutches and with minimal pain. Pain medication remained unchanged. During this period gait speed was increasing indicating functional improvement but trunk sway was also increasing indicating reduced trunk stability und reduced hip muscle control (see Table 5. and figures 6. and 7.). Trunk sway increases with increasing gait speed but the levels of this patient were higher than normal values and continued to increase.(Goutier et al., 2010)

For the next two weeks treatment continued. Figures $6 \& 7$ show that gait speed continued to improve but that trunk sway was also increasing. During these two weeks 
the distance the patient was able to walk without crutches improved slightly to approximately $700 \mathrm{~m}$. It was still not possible to climb stairs without pain.

After four weeks patient and therapist felt that progress had slowed and that a change in treatment approach was necessary.

It was hypothesised that the crutches, which have been shown to reduce hip muscle activity during walking (Sonntag et al., 2000), were having a negative impact on the recovery of hip muscle strength and therefore gait function and trunk sway. As crutches were used for long periods daily, the likelihood of this effect was increased.

We considered that a walking aid which would increase hip abductor and extensor muscle activity whilst providing external hip support may be more appropriate. It was decided that the orthotic garment and strapping system TheraTogs applied to facilitate hip abductor and extensor muscle activity would be an appropriate treatment alternative to crutch walking. It was not clear at this point whether the hip joint which would now be loaded (partial weight-bearing would no longer be possible as arms are not used with TheraTogs) would become more painful.

\section{TheraTogs(Weeks 5-8)}

TheraTogs consists of an inner "suit" worn next to the patient's skin under the clothes. The outer surface is Velcro-sensitive allowing the attachment of elasticated straps. The straps provide a gentle pressure with the aim of improving joint alignment and increasing muscle activity. The orthosis was individually adapted and applied to facilitate hip abductor and extensor muscle activity (Figure 3). The patient applied TheraTogs in the morning when dressing and wore it throughout the day until undressing at night. During this period crutches were not used.

The patient continued with strengthening and stretching exercises twice weekly in water and daily home exercises.

\section{Response to Treatment}

The patient continued initially to walk $500 \mathrm{~m}$ without crutches but now wearing TheraTogs and pain was absent or minimal. After $2-3$ weeks this increased to $1 \mathrm{~km}$ taking 15 - 20 minutes. The patient was fully weight-bearing but still wearing TheraTogs. Pain medication remained unchanged. After 4 weeks the patient could walk approximately $1.5 \mathrm{~km}$ with TheraTogs and distance without aids remained approximately $700 \mathrm{~m}$.

Gait speed continued to improve and at a faster rate than during the crutch walking phase (Table 5 and Figure 6). Trunk sway was reduced compared to the earlier phase although gait speed was increasing. Stair climbing was possible when wearing TheraTogs and using a bannister. It remained painful however and the patient reported that TheraTogs acted more as a hindrance than a help. This may have been because the strap applied to facilitate hip muscle extensor activity opposed hip flexion. 


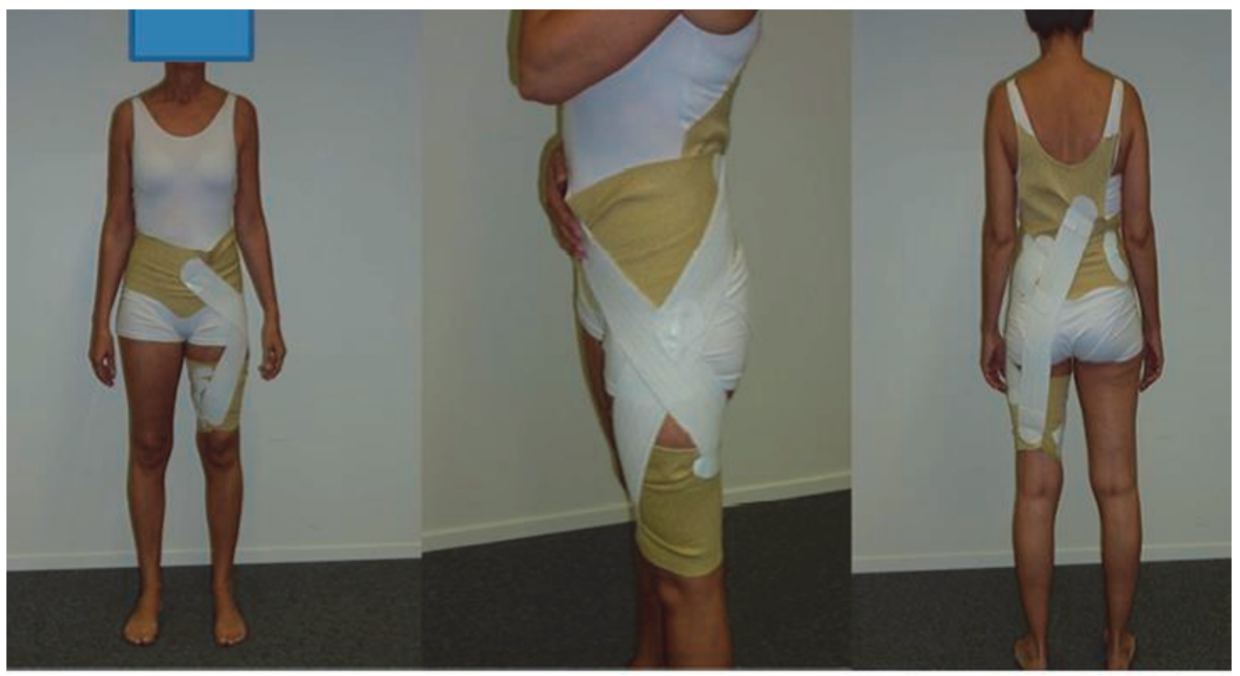

Figure 3. TheraTogs adapted for easy application by the patient herself and applied to facilitate hip abductor and extensor activity.

After two weeks the patient was able to commence cycling with an E-bike and began a twice weekly ride of $5-10 \mathrm{~km}$. Sometimes this would replace the hydrotherapy session. TheraTogs was uncomfortable when cycling. At the end of four weeks gait speed had significantly improved and trunk sway had become more stable. The patient was able to walk longer distances without pain and stair climbing although still uncomfortable was possible. The patient felt that TheraTogs provided stability and support when walking, but was a hindrance when stair climbing and uncomfortable when sitting or cycling. Additionally the patient felt restricted in the clothes she could wear with TheraTogs and was often warm on hot days. As gait speed and trunk-sway had improved and pain was controlled, the patient and therapist decided to progress treatment and attempt to remove walking aids from daily activities and walking.

The question as to whether pelvic stability was improved through improved pelvic muscle activity or through passive support of the orthosis itself remained unclear. Muscle activity when walking without walking aids, with TheraTogs and with crutches using electromyography (EMG) was measured as mentioned above to help clarify this question.

\section{EMG - The Immediate Effects on Muscle Activity}

As shown in Table 4. muscle activity was reduced in all muscle groups when walking with crutches and increased in all muscle groups when walking with TheraTogs. Figure 4 shows typical examples of these effects for the measurements over the standard SENIAM hip abductor position. The nature of EMG means that absolute values on different days cannot be compared. The percentage change between conditions can however be compared. (Konrad, 2006) 
Table 4. EMG activity when walking 1 . Without aids then with crutches and 2. Without aids then with TheraTogs, as a percentage of MVC Mean (SD)

\begin{tabular}{lccccc}
\hline EMG activity as a percentage of Maximum Voluntary Contraction* & & & \\
\hline & $\begin{array}{c}\text { Glut } \\
\text { Maximus }\end{array}$ & $\begin{array}{c}\text { Glut } \\
\text { Medius }\end{array}$ & $\begin{array}{c}\text { Tensor Fascia } \\
\text { Lata }\end{array}$ & Quadriceps & Hamsrtings \\
\hline Without assistive devices & $40.95(7.24)$ & $64.23(10.39)$ & $62.32(18.74)$ & $44.12(11.44)$ & $35.07(3.80)$ \\
With crutches & $13.20(3.48)$ & $29.08(5.97)$ & $36.46(20.22)$ & $19.65(6.89)$ & $23.80(10.29)$ \\
\% change & $-27.75(9.80)$ & $-35.14(12.59)$ & $-25.87(20.80)$ & $-24.47(6.73)$ & $-11.27(9.91)$ \\
Without assistive devices & $58.19(69.19)$ & $45.73(16.49)$ & $29.38(12.72)$ & $20.13(3.22)$ & $39.43(5.00)$ \\
With TheraTogs & $59.87(66.74)$ & $60.85(17.79)$ & $37.63(13.02)$ & $23.07(7.64)$ & $48.14(12.75)$ \\
\% change & $+18.33(26.1)$ & $+36.18(25.19)$ & $+31.41(25.19)$ & $+14.16(22.06)$ & $+24.15(41.35)$ \\
\hline
\end{tabular}

*Measured on different days. Percentage change not absolute values compared.

\section{Mean EMG activity at gluteus medius electrode position walking without aids then with TheraTogs averaged over 9 gait cycles}

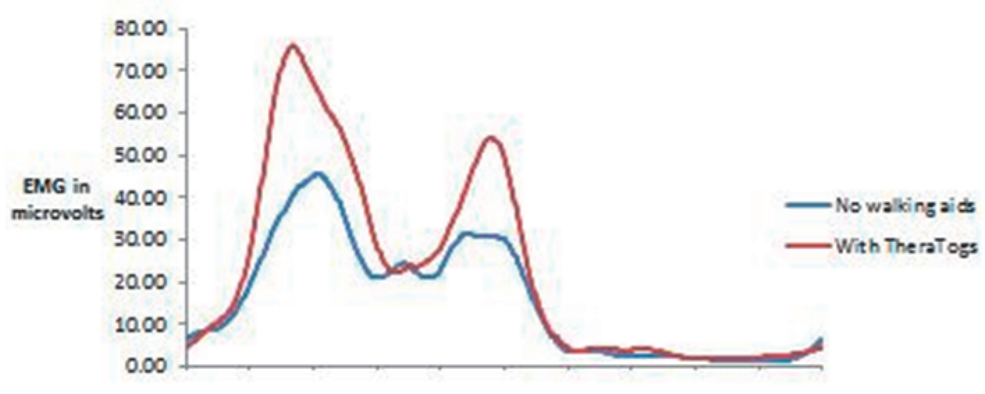

Goit Cycle (heel strike to heel strike)

\section{Mean EMG activity at gluteus medius electrode position walking without aids then with crutches averaged over 9 gait cycles}

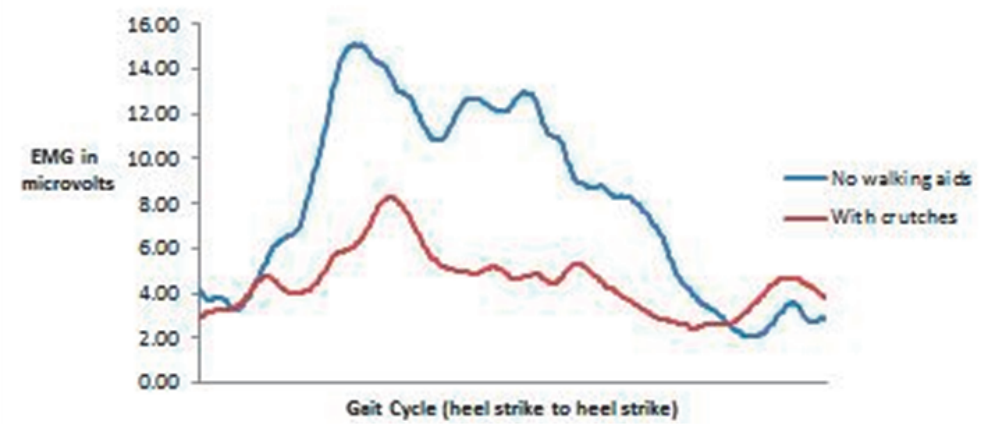

Figure 4. Mean EMG activity over 9 gait cycles at the gluteus medius electrode placement position walking comparing 1. walking without aids with TheraTogs and 2. walking without aids with crutches. 


\section{No Walking Aids (Weeks 9-12)}

The patient now began to walk throughout the day without walking aids. Gait speed and trunk sway continued to be measured twice weekly. Initially strengthening and stretching exercises in water and at home were continued. Cycling with an E-bike 5-10 km once or twice a week and the daily walk was also continued.

\section{Response to Treatment}

After 2 days pain began to increase when walking. After $7-10$ days pain had increased significantly and began to limit function. An acute inflammation at the anatomical level of the greater trochanter was diagnosed as the exposed prosthesis irritated soft tissues. The non-steroidal anti-inflammatory Eterocoxib 90mg to be taken once in the evening, and rest were prescribed. Due to the pain the patient discontinued the E-bike riding and daily walks. Because of high levels of inactivity and the increased pain gait speed reduced and trunk sway increased substantially (Figures 6 and 7). The patient continued to rest as prescribed for the following 10-14 days.

At this point (12 weeks following the commencement of gait rehabilitation) it seemed unlikely that treatment aims of returning to pre-fracture levels of mobility without walking aids would be achieved. Treatment aims of increasing hip muscle strength and mobility to enable walking without aids were not achieved. The patients goals of being able to complete work and leisure tasks without walking aids were also not achieved. The patient was reluctant to return to either crutch walking or TheraTogs on a long term basis. Both of these walking aids had improved functional mobility whilst they were being used but the patient's goal was to walk without assistive devices. It appeared that supporting the hip joint either through partial weight bearing with crutches or through facilitation of muscle activity and the passive support from TheraTogs, were necessary to reduce inflammation and pain when walking. A timeline overview of treatment interventions is presented in Figure 5. 


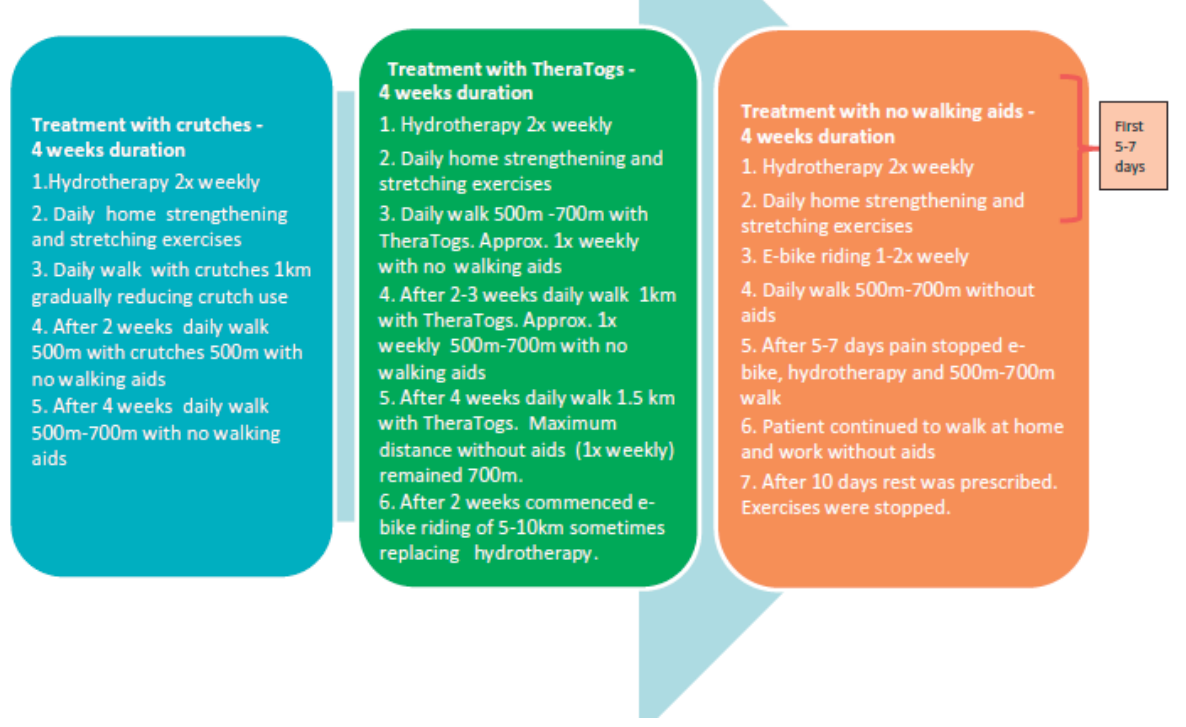

Figure 5. Timeline of treatment interventions

\section{OUTCOME}

\section{Surgical Intervention}

Imaging showed that the trochanter fracture had formed a pseudarthrosis. The shoulder of the hip prosthesis continued to irritate overlying soft tissues and the abductor mechanism, attached to the displaced trochanter fragment could not be normally retrained. Surgery was carried out to change the shaft of the prosthesis, repair the pseudarthrosis with autologous bone grafting and reattachment of the fragment of the trochanter to the femur with osteosynthesis. Thus the gluteal musculature was also reattached. At the time of this report the patient was non weight-bearing. It was expected to take 4-6 months before the procedure was completed and the patient could begin to fully weight bear. 
Table 5. Gait Speed $(\mathrm{m} / \mathrm{s})$ and Trunk Sway $\left(\right.$ degrees $\left.^{2}\right)$ in each phase

\begin{tabular}{|c|c|c|c|}
\hline \multirow{2}{*}{$\frac{\text { Measures }}{\text { Gait Speed m /s }}$} & \multicolumn{3}{|l|}{ Phases } \\
\hline & Phase A & Phase B & Phase C \\
\hline Measurement point & Crutch walking & TheraTogs & No walking aids \\
\hline 1 & 1.01 & 1.25 & 1.29 \\
\hline 2 & 1.01 & 1.19 & 1.51 \\
\hline 3 & 1.03 & 1.21 & 1.10 \\
\hline 4 & 1.23 & 1.43 & 1.14 \\
\hline 5 & 1.16 & 1.38 & 1.10 \\
\hline 6 & 1.16 & 1.48 & 1.05 \\
\hline 7 & 1.13 & 1.48 & 1.19 \\
\hline 8 & 1.16 & 1.38 & 1.14 \\
\hline Mean (SD) & $1.11(0.08)$ & $1.35(0.11)$ & $1.19(0.14)$ \\
\hline Median (IQR) & $1.14(0.14)$ & $1.38(0.25)$ & $1.14(0.17)$ \\
\hline \multicolumn{4}{|c|}{$\begin{array}{l}\text { Trunk Sway - Total angle } \\
\text { area in degrees }^{2} \\
\text { Measurement point }\end{array}$} \\
\hline 1 & 59.04 & 71.96 & 100.49 \\
\hline 2 & 48.08 & 85.43 & 88.12 \\
\hline 3 & 49.04 & 61.36 & 85.63 \\
\hline 4 & 73.86 & 67.63 & 74.82 \\
\hline 5 & 59.10 & 68.38 & 101.70 \\
\hline 6 & 107.11 & 61.28 & 104.12 \\
\hline 7 & 89.90 & 81.74 & 87.11 \\
\hline 8 & 67.95 & 78.30 & 53.26 \\
\hline Mean (SD) & $69.26(20.51)$ & 72.01 (9.06) & $86.90(16.82)$ \\
\hline Median (IQR) & $63.52(34.35)$ & 70.17 (17.96) & 87.61 (23.88) \\
\hline
\end{tabular}

$\mathrm{SD}=$ Standard Deviation. $\mathrm{IQR}=$ Interquartile Range 


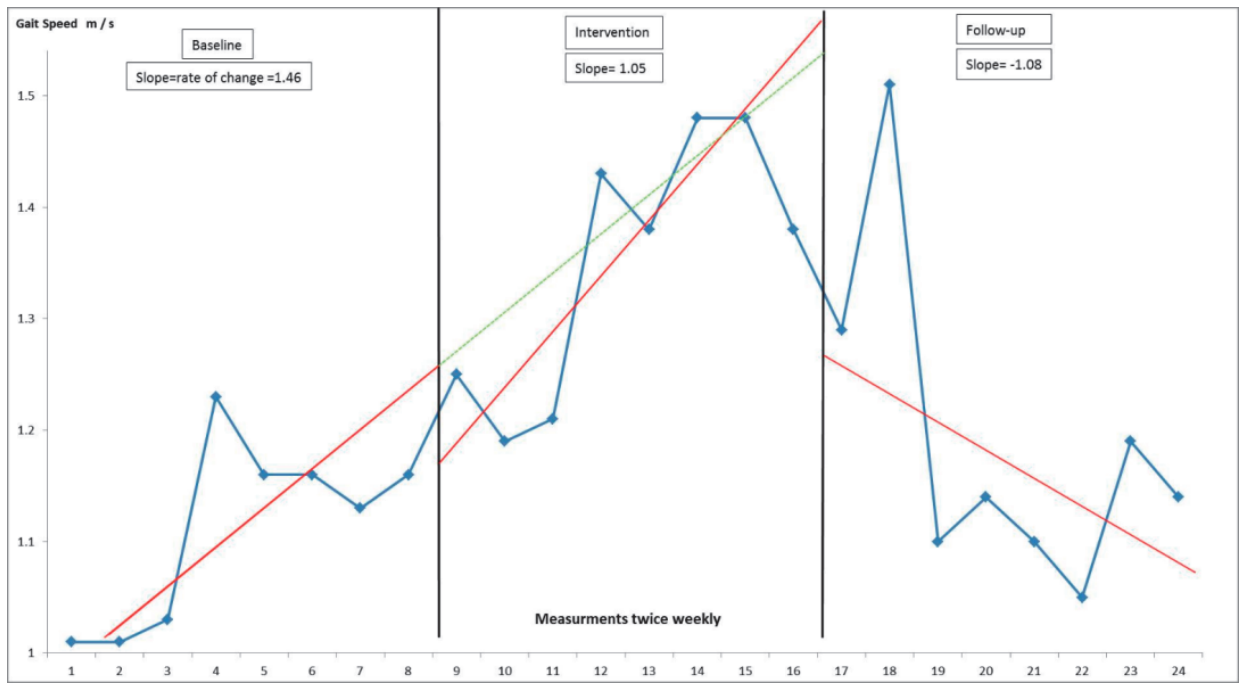

Figure 6. Gait speed - twice weekly measurements during gait rehabilitation with crutches, TheraTogs and without walking aids. Red line is the calculated "Celeration" line indicating rate of change in each phase. The green line in the TheraTogs phase indicates expected values in TheraTogs phase if rate of change from crutch walking had continued.

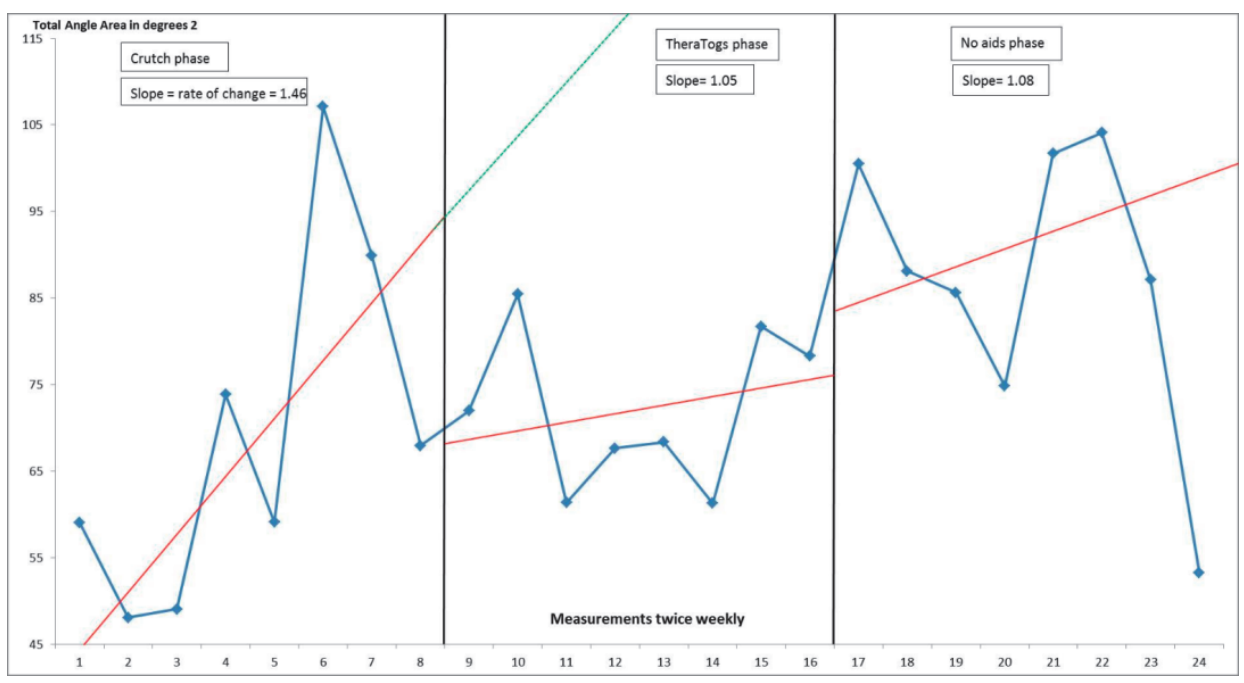

Figure 7. Trunk-sway twice weekly measurements during gait rehabilitation with crutches, TheraTogs and without walking aids. Increasing values indicate greater instability. Red line is the calculated "Celeration" line indicating rate of change in each phase. The green line in the TheraTogs phase indicates expected values in TheraTogs phase if rate of change from crutch walking had continued. 


\section{DISCUSSION}

\section{Effects of the Walking Aids during Rehabilitation.}

To our knowledge this is the first detailed case to document the specific influence of walking aids over a prolonged period on functional recovery in orthopedic rehabilitation.

\section{Gait Speed and Trunk Sway}

Table 5 and Figures 6 and 7 show the changes in gait speed and trunk sway as the patient underwent gait rehabilitation using different walking aids. Gait speed increased during crutch walking. It continued to improve with TheraTogs at a faster rate of change (steeper slope) and reduced when walking with no-aids. Gait speed (m/s) mean(SD) changed from 1.11(0.08) in crutch phase, to 1.35 (0.11) with TheraTogs. According to Alley et al. (Alley et al., 2011) who investigated meaningful improvement in gait speed in 217 women following hip fracture, this represents a "substantial functional improvement". This was mirrored in the activity levels when walking with TheraTogs. A daily walk of $500 \mathrm{~m}$ to $1 \mathrm{~km}$ was continued as during crutch walking but the patient was fully weight bearing. 1-2 weekly bike rides of 5-10 km were also done on a regular basis. During the no walking aids phase gait speed reduced again to $1.19(0.14) \mathrm{m} / \mathrm{s}$. The patients function was substantially reduced, shown by the necessity to stop activity such as daily walks and e-bike riding.

Trunk sway increased steadily indicating increasing pelvic and balance instability during the crutch walking phase. In healthy subjects increasing gait speed is associated with increased trunk sway.(Goutier et al., 2010) It could be argued therefore that the increased values were due to increasing gait speed as values began and remained well above normal. Normal values for the average gait speed obtained by this patient when crutch walking vary between 18 and 31.62 degrees $^{2}$. (Goutier et al., 2010) This value in our patient was $69.26(20.51)$ degrees $^{2}$ indicating instablity and consistent with the generalised weakness around the hip. Increasing trunk sway is associated with an increased risk of falling. (Bischoff-Ferrari et al., 2006; Dingwell \& Marin, 2006; Goutier et al., 2010; van lersel, Ribbers, Munneke, Borm, \& Rikkert, 2007)

During the TheraTogs phase the values became less variable, exhibiting fewer extremes of movement and indicating improved stability. The rate of increase was much reduced compared to crutch walking, even though gait speed increased. In the no walking aids phase trunk sway again began to increase at a faster rate indicating greater instability despite reducing gait speed. 


\section{Muscle Activity.}

When walking with crutches, muscle activity was reduced in all muscle groups compared to walking with no aids, confirming the findings of Sonntag D et al. (Sonntag et al., 2000). Despite this, gait speed increased. A possible explanation is that the unloading which occurs at the hip joint when walking with crutches (Bateni \& Maki, 2005) not only caused an ongoing reduction in muscle activity, but also enabled spontaneous recovery of the affected structures resulting in increased gait speed. However, given the importance of hip abductor control in postural stability (Gribble \& Hertel, 2004; Negahban et al., 2013), the constant inhibition of this activity in this phase may have been responsible for the increasing trunk sway.

The immediate effect of TheraTogs on muscle activity compared to walking without aids was an increased activity in all muscle groups with the largest effects at the hip abductor muscles (gluteus medius electrode position) and hamstrings. The mechanism by which TheraTogs facilitates muscle activity has not been determined. It may be that propriceptive input from the elasticated straps facilitates muscle activity or that the passive correction of biomechanical aligment during walking "normalises" muscle length allowing improved contraction. Due to the avulsion fracture of the greater trochanter the biomechanical source of the abductor activity remained unclear but the functional relevance was demonstrated through the improved walking speed and trunk sway. The activity could have been the result of surface EMG crosstalk (EMG signal generated by a nearby muscle) (Farina, Merletti, Indino, \& Graven-Nielsen, 2004) (Beck, DeFreitas, \& Stock, 2010) for example from tensor fascia lata, or it may have arisen from muscular contractile elements which can fuse with the gluteal aponeurosis.(Netter et al., 2014)

\section{Possible Implications for Clinical Practice}

Walking with TheraTogs increased muscle activity, gait speed improved at a faster rate than crutch walking although the hip was not unloaded through partial weight bearing and trunk stability improved. The facilitation of muscle activity through TheraTogs may have accounted for increasing gait speed in this phase and stabilisation of trunk sway due to increased activity in hip abductors.

However the gains made during the TheraTogs phase were not maintained when walking without aids. The influence of the exposed prosthesis may have prevented longterm benefits. When walking without aids gait speed and trunk stability deteriorated. Possibly because the hip was not supported either through unloading or due to facilitation of muscle activity, the exposed shoulder of the prosthesis caused increased irritation and inflammation of overlying soft tissues during walking. This could explain the increased pain, reduction in trunk stablity (pain inhibition of muscle activity) and reduction of gait speed. All outcome measures deteriorated when walking with no aids and 
daily activity was reduced. Improvements in strength and function made during treatment with TheraTogs were insufficient to ensure independent support of the joint with no aids. These changes could not prevent irritation of overlying soft tissues and the escalation of inflammation and pain.

Ultimately the patients goal of walking independently without aids was not achieved through conservative interventions. As the patient was reluctant to use walking aids long-term, surgery was considered necessary to restore function.

Overall the results measured with this patient suggest that TheraTogs may offer an attractive alternative to crutch walking in orthopedic patients particularly if assistance is needed in the progression to full weight bearing. TheraTogs appeared to provide adequate support to the recovering structures whilst facilitating muscle activity.This enabled an increased recovery rate of all measured parameters, compared to walking with crutches. In this patient these changes were not maintained when progressing to walking with no aids. However the exposed prosthesis irritating overlying structures caused a more challenging situation than occurs in many patients following orthopedic hip surgery.

As up to $20 \%$ of patients demonstrate a failure of the hip abductor mechanism following total hip replacement (Odak \& Ivory, 2013) and as post-operative fracture of the greater trocahnter is not uncommon (Pritchett, 2001) this functional improvement may be particularly important for this patient group who are often treated conservatively (Odak \& Ivory, 2013).

As hand-held assistive devices have been shown to be associated with increased risk of falling, possibly due to tripping or by diverting attentional resources (Bateni \& Maki, 2005), devices such as TheraTogs may avoid these drawbacks. Problems due to repetitive stress on upper extremity joints are also avoided.

It is important to emphasize that these results have only been confirmed in one patient and cannot be generalized. Research to investigate the long term impact of different walking aids on the functional recovery of gait and balance are needed.

\section{ACKNOWLEDGEMENTS}

The authors would like to thank Hans-Peter Karrer from the Bildungszentrum Gesundheit Basel Stadt for his support.

\section{DECLARATION OF INTEREST}

The authors report no declarations of interest. 


\section{REFERENCES}

Abd El-Kafy, E. M. (2014). The clinical impact of orthotic correction of lower limb rotational deformities in children with cerebral palsy: a randomized controlled trial. Clin Rehabil, 28: 1004-1014

Alley, D. E., Hicks, G. E., Shardell, M., Hawkes, W., Miller, R., Craik, R. L., . . Magaziner, J. (2011). Meaningful improvement in gait speed in hip fracture recovery. J Am Geriatr Soc, 59(9), 1650-1657.

Allum, J. H., \& Carpenter, M. G. (2005). A speedy solution for balance and gait analysis: angular velocity measured at the centre of body mass. Curr Opin Neurol, 18(1), 15-21.

Archdeacon, M., Ford, K. R., Wyrick, J., Paterno, M. V., Hampton, S., Ludwig, M. B., \& Hewett, T. E. (2008). A prospective functional outcome and motion analysis evaluation of the hip abductors after femur fracture and antegrade nailing. J Orthop Trauma, 22(1), 3-9.

Bateni, H., \& Maki, B. E. (2005). Assistive devices for balance and mobility: benefits, demands, and adverse consequences. Arch Phys Med Rehabil, 86(1), 134-145.

Beck, T. W., DeFreitas, J. M., \& Stock, M. S. (2010). Cross-talk among monopolar surface electromyographic signals from the superficial quadriceps femoris muscles. Electromyogr Clin Neurophysiol, 50(5), 245-250.

Bischoff-Ferrari, H. A., Conzelmann, M., Stähelin, H. B., Dick, W., Carpenter, M. G., Adkin, A. L., . . Allum, J. H. (2006). Is fall prevention by vitamin D mediated by a change in postural or dynamic balance? Osteoporos Int, 17(5), 656-663.

Borrelli, J., Jr., Ricci, W. M., Anglen, J. O., Gregush, R., \& Engsberg, J. (2006). Muscle strength recovery and its effects on outcome after open reduction and internal fixation of acetabular fractures. J Orthop Trauma, 20(6), 388-395.

Corporaal, S. H., Gensicke, H., Kuhle, J., Kappos, L., Allum, J. H., \& Yaldizli, Ö. (2013). Balance control in multiple sclerosis: correlations of trunk sway during stance and gait tests with disease severity. Gait Posture, 37(1), 55-60.

Dingwell, J. B., \& Marin, L. C. (2006). Kinematic variability and local dynamic stability of upper body motions when walking at different speeds. J Biomech, 39(3), 444-452.

Farina, D., Merletti, R., Indino, B., \& Graven-Nielsen, T. (2004). Surface EMG crosstalk evaluated from experimental recordings and simulated signals. Reflections on crosstalk interpretation, quantification and reduction. Methods Inf Med, 43(1), 30-35.

Garcia, F. L., Picado, C. H., \& Nogueira-Barbosa, M. H. (2010). Sonographic evaluation of the abductor mechanism after total hip arthroplasty. J Ultrasound Med, 29(3), 465-471.

Goutier KM, Jansen SL, Horlings CG, Küng UM, Allum JH 2010 The influence of walking speed and gender on trunk sway for the healthy young and older adults. Age Ageing 39: 647-650.

Gribble, P. A., \& Hertel, J. (2004). Effect of hip and ankle muscle fatigue on unipedal postural control. J Electromyogr Kinesiol, 14(6), 641-646.

Hermens, H. J., Freriks, B., Disselhorst-Klug, C., \& Rau, G. (2000). Development of recommendations for SEMG sensors and sensor placement procedures. J Electromyogr Kinesiol, 10(5), 361-374.

Hol, A. M., van Grinsven, S., Lucas, C., van Susante, J. L., \& van Loon, C. J. (2010). Partial versus unrestricted weight bearing after an uncemented femoral stem in total hip arthroplasty: recommendation of a concise rehabilitation protocol from a systematic review of the literature. Arch Orthop Trauma Surg, 130(4), 547-555.

Horlings, C. G., Küng, U. M., van Engelen, B. G., Voermans, N. C., Hengstman, G. J., van der Kooi, A. J., . . . Allum, J. H. (2009). Balance control in patients with distal versus proximal muscle weakness. Neuroscience, 164(4), 1876-1886.

Howell, G. E., Biggs, R. E., \& Bourne, R. B. (2001). Prevalence of abductor mechanism tears of the hips in patients with osteoarthritis. J Arthroplasty, 16(1), 121-123.

Hung, W. W., Egol, K. A., Zuckerman, J. D., \& Siu, A. L. (2012). Hip fracture management: tailoring care for the older patient. JAMA, 307(20), 2185-2194.

Klemetti R, Steele KM, Moilanen P, Avela J, Timonen J 2014 Contributions of individual muscles to the sagittaland frontal-plane angular accelerations of the trunk in walking. J Biomech, 47: 2263-2268. 
Konrad, P. (2006). The ABC of EMG. A Practical Introduction to Kinesiological Electromyography.: Noroxon.

Maguire, C., Sieben, J. M., Frank, M., \& Romkes, J. (2010). Hip abductor control in walking following stroke -the immediate effect of canes, taping and TheraTogs on gait. Clin Rehabil, 24(1), 37-45.

Masonis, J. L., \& Bourne, R. B. (2002). Surgical approach, abductor function, and total hip arthroplasty dislocation. Clin Orthop Relat Res(405), 46-53.

Negahban, H., Etemadi, M., Naghibi, S., Emrani, A., Shaterzadeh Yazdi, M. J., Salehi, R., \& Moradi Bousari, A. (2013). The effects of muscle fatigue on dynamic standing balance in people with and without patellofemoral pain syndrome. Gait Posture, 37(3), 336-339.

Netter, H, \& Frank. (2014). Atlas of Human Anatomy (6th Edition ed.). Philadelphia: Elsevier Saunder.

Neumann, D. A. (1998). Hip abductor muscle activity as subjects with hip prostheses walk with different methods of using a cane. Phys Ther, 78(5), 490-501.

Odak, S., \& Ivory, J. (2013). Management of abductor mechanism deficiency following total hip replacement. Bone Joint J, 95-B(3), 343-347.

Piva, S. R., Teixeira, P. E., Almeida, G. J., Gil, A. B., DiGioia, A. M., Levison, T. J., \& Fitzgerald, G. K. (2011). Contribution of hip abductor strength to physical function in patients with total knee arthroplasty. Phys Ther, 91(2), 225-233.

Pritchett, J. W. (2001). Fracture of the greater trochanter after hip replacement. Clin Orthop Relat Res(390), 221-226.

Richards, A., Morcos, S., Rethlefsen, S., \& Ryan, D. (2012). The use of TheraTogs versus twister cables in the treatment of in-toeing during gait in a child with spina bifida. Pediatr Phys Ther, 24: 321-326.

Robbins, C. E., Casey, D., Bono, J. V., Murphy, S. B., Talmo, C. T., \& Ward, D. M. (2014). A multidisciplinary total hip arthroplasty protocol with accelerated postoperative rehabilitation: does the patient benefit? Am J Orthop (Belle Mead NJ), 43(4), 178-181.

Sonntag, D., Uhlenbrock, D., Bardeleben, A., Kading, M., \& Hesse, S. (2000). Gait with and without forearm crutches in patients with total hip arthroplasty. Int J Rehabil Res, 23(3), 233-243.

Tayrose, G., Newman, D., Slover, J., Jaffe, F., Hunter, T., \& Bosco lii, J. (2013). Rapid Mobilization Decreases Length-of-Stay in Joint Replacement Patients. Bull Hosp Jt Dis 71(3), 222-226.

van lersel, M. B., Ribbers, H., Munneke, M., Borm, G. F., \& Rikkert, M. G. (2007). The effect of cognitive dual tasks on balance during walking in physically fit elderly people. Arch Phys Med Rehabil, 88(2), 187-191.

Westerman, R. W., Hull, P., Hendry, R. G., \& Cooper, J. (2008). The physiological cost of restricted weight bearing. Injury, 39(7), 725-727. doi: 10.1016/j.injury.2007.11.007

Williams, B. S., \& Cohen, S. P. (2009). Greater trochanteric pain syndrome: a review of anatomy, diagnosis and treatment. Anesth Analg, 108(5), 1662-1670.

Zhao, Y., Chen, S. Y., Lee, Y. C., \& Wu, N. (2013). Clinical and economic characteristics of hip fracture patients with and without muscle atrophy/weakness in the United States. Arch Osteoporos, 8: 127. 



\section{Chapter}

How to improve walking, balance and social participation following stroke: a comparison of the long term effects of two walking aids - canes and an orthosis TheraTogs - on the recovery of gait following acute stroke. A study protocol for a multicentre, single blind, randomised control trial. 


\section{ABSTRACT}

Background Annually, some 9000 people in Switzerland suffer a first time stroke. Of these $60 \%$ are left with moderate to severe walking disability. Evidence shows that rehabilitation techniques which emphasise activity of the hemiplegic side increase ipsilesional cortical plasticity and improve functional outcomes. Canes are commonly used in gait rehabilitation although they significantly reduce hemiplegic muscle activity. We have shown that an orthosis "TheraTogs" (a corset with elasticated strapping) significantly increases hemiplegic muscle activity during gait. The aim of the present study is to investigate the long term effects on the recovery of gait, balance and social participation of gait rehabilitation with TheraTogs compared to gait rehabilitation with a cane following first time acute stroke.

Methods Multi-centre, single blind, randomised trial with 120 patients after first stroke. When subjects have reached Functional Ambulation Category 3 they will be randomly allocated into TheraTogs or cane group. TheraTogs will be applied to support hip extensor and abductor musculature according to a standardised procedure. Cane walking held at the level of the radial styloid of the sound wrist. Subjects will walk throughout the day with only the assigned walking aid. Standard therapy treatments and usual care will remain unchanged and documented. The intervention will continue for five weeks or until patients have reached Functional Ambulation category 5. Outcome measures will be assessed the day before begin of intervention, the day after completion, 3 months, 6 months and 2 years. Primary outcome: Timed "up and go" test, secondary outcomes: peak surface EMG of gluteus maximus and gluteus medius, activation patterns of hemiplegic leg musculature, temporo-spatial gait parameters, hemiplegic hip kinematics in the frontal and sagittal planes, dynamic balance, daily activity measured by accelerometry, Stroke Impact Scale.

Results Significance levels will be 5\% with 95\% Cl's. IntentionToTreat analyses will be performed. Descriptive statistics will be presented.

Discussion This study could have significant implications for the clinical practice of gait rehabilitation after stroke, particularly the effect and appropriate use of walking aids. The results could be important for the development of clinical guidelines and for the socio-economic costs of post-stroke care.

Keywords. Stoke, gait, canes, TheraTogs, EMG 


\section{BACKGROUND}

Stroke is the leading cause of acquired disability in adults. [1] In Switzerland approximately 9000 people a year suffer a first time stroke. [2] Of those patients who survive the acute phase, between 20 and $30 \%$ are unable to walk. [3] Those who can are often left with moderate to severe walking disability and reduced gait speeds. [3,4] The risk of falling increases - a recent study indicating that at 3 months $28 \%$ of stroke patients have fallen. [5] The resulting disability has an enormous socioeconomic impact on patients, families and health service providers. [6,7]

Rehabilitation methods which improve balance and gait are crucial for the quality of life of stroke victims and to reduce the ongoing cost of long term care. Studies have shown that early rehabilitation in specialised settings e.g. stroke units results in better functional outcome than in non-specialised units. [8,9]. It has been suggested that these better results are achieved partly due to enhanced staff awareness of the importance of mobility thus preventing secondary complications such as loss of cardiovascular fitness or muscle weakness. The physical environment is also likely to be adapted enabling more independent movement. $[10,11]$. Current rehabilitation methods which aim to improve motor control during walking do not appear to deliver additional improvements $[12,13]$ A recent meta-analysis showed that only cardiorespiratory physical fitness training provides robust evidence for a benefit to walking ability after stroke. Repetitive task training also appeared to have some effect. Motor and neurophysiological approaches did not demonstrate a positive effect on walking recovery [13] This suggests that underlying mechanisms responsible for the recovery of motor co-ordination and control of walking following stroke are not significantly influenced by current therapy methods. These findings are further illustrated by studies which show that long term improvements in gait function occur in the absence of improvements in muscle co-ordination patterns [14] or improved kinematic or kinetic gait profiles [15]. Buurke et al. [14] concluded that "functional gait improvements may be more related to compensatory strategies ..than by restitution of muscle co-ordination patterns in the affected leg." Kautz et al. [16] concluded that "There is no evidence of improved locomotor co-ordination post intervention. The increased walking and pedalling speed were achieved by a more proficient use of the same impaired pattern without EMG timing changes, likely because of increased strength and endurance post intervention." We suggest that improvements in gait function due to current rehabilitation methods are predominantly achieved through more efficient use of abnormal movement patterns. This may be a reason for the generally low level of independence and function achieved following stroke.

Spontaneous recovery which occurs within the first weeks post stroke largely defines long term functional outcome. [17] Recent studies investigating changes in the cerebral cortex following focal injury have indicated that cortical plasticity and neuronal growth that occurs early after infarct may "underlie the brain`s self-repair process". [18, 
19] Evidence shows that this early cortical plasticity is an important factor in the spontaneous recovery of motor control which predicts long term outcome.[18,19,20,21, 22] We suggest that it is this process which is inadequately influenced by current rehabilitation methods. The recovery of motor skills following stroke relies on the brains ability to reorganise its neuronal control of movement. $[19,20]$ Reorganisation can occur within and between cortical networks both within the lesioned and non-lesioned hemisphere. $[21,18]$ Rossini et al. stated that "Reorganization phenomena following ischemic stroke observed so far, may be classified into two main groups: overactivation of areas belonging to the neural network for a specific task - or activation of unusual areas that attempt to replace the function of the damaged tissue." [18] The overactivation of the original neural network is primarily activity in the lesioned hemisphere. Following stroke cortical activity has been recorded in both lesioned and non-lesioned hemispheres during movement. [21,23] Increasingly findings show that increased activity in the lesioned hemisphere correlates with better recovery and improved motor performance $[23,24,25,26,27]$ Conversely increased activity in the contralesional hemisphere is associated with poorer motor recovery of the hemiplegic limb.[ 26,27] It appears that improved motor recovery occurs when the brain is able to make use of the original neural network to control movement. When new networks are formed for example in the unaffected hemisphere, motor recovery is reduced. The authors suggest that activity in the original network represents "true" recovery. The recruitment of new networks may represent the learning of compensatory movement strategies associated with poorer functional outcome.

Findings in favour of this hypothesis have been demonstrated in both upper and lower limbs. $[25,26,27]$. As post-injury behavioural experience is critical to the reassembly of adaptive networks and strongly influences cortical reorganisation $[22,28]$ these results indicate that treatment approaches which promote increased plasticity in the lesioned hemisphere will promote greater functional recovery. This assumption is supported by a recent meta-analysis [21] which examined whether participating in stroke rehabilitation which emphasised use of the paretic upper limb was associated with increased cortical activity of the lesioned hemisphere and consequently better function. The review concluded that neural changes in the sensorimotor cortex of the lesioned hemisphere were achieved with rehabilitation interventions that emphasized use of the paretic upper limb and resulted in improved functional motor gains.

Nudo et al. [28] demonstrated with squirrel monkeys that forced retraining of skilled hand use prevented loss of hand territory representation adjacent to the infarct and in some instances the representations expanded. This functional reorganization was accompanied by recovery of skilled hand movements. Control monkeys with identical lesions who did not receive therapy lost paretic hand representation und function.

In general these results suggest that therapies which emphasise use of the hemiplegic side promote plasticity in the lesioned hemisphere through emphasising the use of previously established neural networks and are associated with improved function. It 
may be that therapies which promote compensatory movement or reduce activity of the hemiplegic limb inhibit plasticity in the lesioned hemisphere and promote the development of new neural networks associated with reduced function.

To date therapies which emphasise use of the hemiplegic limb have been confined to the upper extremity $[29,30]$ and have not been applied during gait rehabilitation following stroke. Canes are very commonly used post stroke although studies have consistently shown a significant reduction in surface electromyography (EMG) activity in all muscle groups on the side contralateral to cane use in both stroke and non-stroke patients. [14,31,32,33]. In light of the factors which increase plastic reorganisation in the lesioned hemisphere, namely interventions which emphasise use of the paretic limb, the effect of canes which reduce activity in the hemiplegic side may be to inhibit activity in the original neural networks responsible for lower limb control resulting in poorer walking function.

In gait rehabilitation, attention should also be paid to the optimal restoration of balance. In relation to balance, evidence shows that balance control does not occur automatically at spinal cord and brainstem level as has previously been thought, but rather is highly influenced by cortical activity and cognitive control.[34,35,36]. Two main types of balance strategies are recognised - fixed support or change of support strategies. $[34,35]$ Fixed support strategies are used when no stepping or reaching activities are needed to maintain balance. Rotatory torques are generated through muscle activity primarily around the hip and ankle. Change of support strategies are used in challenging conditions when stepping or reaching reactions are necessary to maintain equilibrium. Fixed support strategies use less cognitive resources than change in support.[34,35] Elderly people or subjects with poor balance use more change in support strategies and therefore more cognitive resources than younger, healthy subjects to maintain equilibrium under the same conditions.

The authors suggest that balance rehabilitation should attempt to restore balance strategies used by healthy individuals - namely fixed support responses requiring fewer cognitive resources in unchallenging situations. However cane use increases the base of support when walking through use of the arms. This strategy may emphasise use of cortical networks used for reaching "change of support" reactions. It emphasises use of cognitive resources on safe level ground and reduces the use of "fixed support" strategies. The authors suggest this leads to a long term reduction of automatic balance responses. It may be that because cognitive resources are needed in safe, level environments fewer additional resources are available for more difficult conditions such as walking outside or on public transport. The long term effects of canes on balance recovery and functional gait following stroke has to our knowledge been investigated in one study [37]. Balance recovery and community participation were shown to be reduced.

Taking all of these considerations together, the authors suggest that an optimal walking aid for post stroke gait rehabilitation would provide enough support to enable independent early walking without reducing hemiplegic muscle activity or inhibiting the 
use of balance reactions. The immediate effect of an elasticised orthotic walking aid (TheraTogs) on hemiplegic hip abductor activity has been previously investigated by the authors. [38] Activity in gluteus medius was increased by $16.5 \%$ compared to walking without aids when walking with TheraTogs (with cane use activity in gluteus medius was reduced by $22 \%$ compared to walking without aids). The increased activity with TheraTogs may be due to increased proprioceptive input provided by the orthosis or to the physical shortening of the muscle leading to increased overlap between the actin and myosin filaments and consequently a stronger contraction.

The authors hypothesize that cane use will inhibit activity on the hemiplegic side leading to reduced ipsilesional cortical activity and poorer long term functional gait outcomes. In contrast we hypothesize that TheraTogs will increase activity on the hemiplegic side during walking leading to increased ipsilesional cortical activity and improved long term functional gait outcomes.

We further hypothesize that cane use will inhibit the use of normal balance reactions leading to reduced balance recovery and poorer social participation. As no external support is provided with the TheraTogs orthosis the use of automatic balance responses will not be inhibited during walking. This will result in improved balance recovery and social participation.

The aim of this study is to investigate the long term effects of canes and TheraTogs on the recovery of motor control and co-ordination, gait, daily activity, balance and social participation when used in early gait rehabilitation following stroke.

\section{METHODS}

\section{Design and setting}

This study is a multi-centred single blind, randomised, control trial with parallel design.

Subjects will be recruited from the neurological rehabilitation department of 3 participating Swiss hospitals: Felix Platter Spital Basel, Kantonspital Luzern Neurorehabiltaton and Reha Clinic Zurzach. Each department is a dedicated unit specialising in the rehabilitation of post acute neurological patients. Ethical approval has been obtained from the cantonal ethics committees.

\section{In- and exclusion criteria}

All subjects (1) will be patients with hemiplegia following a first unilateral stroke, (2) will score at least level 3 on the Functional Ambulation Category (FAC) [39] (able to walk unaided on even ground but requiring verbal prompts and stand-by help without body contact) and (3) must have been independent walkers prior to insult without walking aids. (4)Subjects will have a Mini Mental State [40] score of 22 or above, (5)will have no ortho- 
paedic or other neurological conditions that could limit walking ability, (6)have no gross visuospatial or visual field deficits and (7)will have no medical contraindications to walking.

\section{Recruitment}

Potential subjects will be identified by rehabilitation staff at the participating hospitals. Suitability for participation will be checked and confirmed by the researchers. Signed, informed consent will be obtained from subjects before inclusion and randomisation. Subjects will be randomised into TheraTogs (intervention) and cane (control) group (see below). Intervention will begin when patients have reached level 3 on the FAC.

\section{Intervention details}

Interventions will be applied for five weeks. If patients become independent walkers before this time (FAC 5) the intervention will be discontinued. If patients are discharged before this time and have not reached FAC 5 where possible the home carer will be instructed in the application of TheraTogs and the intervention will continue for 5 weeks as planned. TheraTogs will be applied as part of the washing/dressing routine in the morning by therapists or nursing staff instructed in the standard application. Subjects will walk throughout the day until preparing for bed with the prescribed walking aid. Standby assistance only will be provided during walking when necessary for safety. No other form of walking aid will be used for the duration of the study intervention. Foot or foot/ankle orthoses will remain unchanged and be worn as usual. All other forms of therapy (frequency and type) will remain unchanged and documented. Patients will receive usual care. When therapists feel that TheraTogs hinders treatment sessions the application may be removed for the duration of the session. It must be immediately reapplied with the standard application following treatment. When necessary TheraTogs may be removed for sleeping in the afternoon. It will be reapplied with the standard application on waking.

TheraTogs is worn directly on the skin. Attached to the basic suit hip abductor support [38] will consist of two broad straps attached 1.to the anterior torso, pulled downwards across the abductors towards and attached to the posterior aspect of the hemiplegic leg and , 2. to the posterior aspect of the torso pulled downwards to cross the abductors and attach to the anterior aspect of the hemiplegic leg For hip extension one wide strap will be attached to the top of the pelvic rim on the non-hemiplegic side, pulled downwards and laterally passing across the buttock and towards the anterior aspect of the hemiplegic thigh attaching laterally. (Fig 1.) Standard application training sessions will be provided to all staff before study begin. Written and photographic instructions will be provided. TheraTogs suits will be marked to ensure consistent application. 


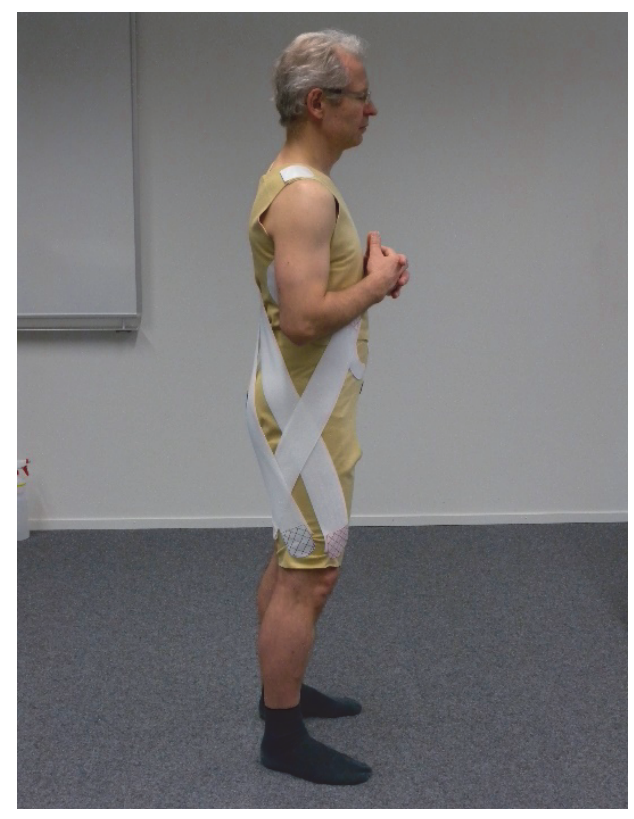

Figure 1. Standard application TheraTogs

In the control group cane walking will take place with cane held at the level of the radial styloid of the sound wrist. Occurrences of non-adherence to the protocol will be documented in subject notebooks.

\section{Measures}

Outcome measures will be taken at baseline, defined as the day before intervention begin, the day after intervention is completed (max. 5 weeks), 3 months, 6 months and 2 years after intervention begin.

\section{Baseline descriptives}

At baseline descriptive variables for each patient including age, sex area and type of infarct, side of stroke, time since stroke, height and weight will be recorded.

\section{Primary outcome}

The primary outcome measure will be the time taken to complete the Timed "Up and Go" Test. [41] This is a basic test for functional mobility which has good reliability and validity. [41,42] The seat height will be $65 \%$ of the subjects leg length and subjects will turn towards the unaffected side [43] 


\section{Secondary outcomes}

The following secondary outcomes will be measured during walking and during sit to stand (in "Timed up and go"):

Peak surface EMG measurement of gluteus maximus and gluteus medius muscle (TMS International, Enschede, Holland);

Activation patterns of gluteus maximus, gluteus medius, vastus lateralis, semitendinosis, gastrocnemius and tibialis anterior; the peak SEMG amplitude of the maximum voluntary contraction of the unaffected gluteus maximus and medius will also be measured.

Temperospatial gait parameters - gait speed, cadence, step length, stride length, stance phase and swing phase of both legs; hip kinematics in the frontal and sagittal planes using twin-axis electrogoniometers (Biometrics Ltd UK,USA SG 150).

An intergrated system The Porti-system with a Polybench software package (TMS International, Enschede, Holland) will be used to simultaneously measure these parameters together with a synchronised camcorder with a sagittal view.

Dynamic balance will be simultaneously measured using two angular velocity sensors (fibreoptic gyroscopes) of the SwayStar balance system (Balance International Innovations GmbH, Iseltwald, Switzerland). [44,45]

Muscle strength of hemiplegic and non-hemiplegic hip abductors will be measured using a hand held dynamometer.

Daily activity during the intervention will be measured using an accelerometer (Aipermon $\mathrm{GmbH}$, Germany). Activity modes and accelerometer detection accuracy have been validated. [46]

Social participation will be measured using The Stroke Impact Scale (SIS)[47] Validated German, French and Italian versions will be used. $[47,48]$ The Stroke Impact Scale is a stroke specific evaluative instrument that measures the impact of stroke in multiple domains including physical, emotional, memory/thinking, communication and social participation. It is a face-to-face interviewer administered instrument that takes 15 to 20 minutes to conduct. [47]

"Usual care" will be documented daily for each patient in a specifically designed questionnaire. Each 10 minute treatment block will be classified into 1 of 5 categories. (See attachment "Documentation Standard Therapy.").

Technical details about the measurement of primary and secondary outcome variables are explained below.

\section{Testing Procedure}

Physical measurements take place and data collection is performed in a specific, standardised order, conform to the following protocol: 
1. Hip abductor muscle strength on both sides of the body will be measured using a hand-held dynamometer. Each test will be performed three times for each leg starting with the healthy leg then alternating. Subjects will rest for 30 seconds between trials. Subjects will lie in supine on an examination plinth. Two Velcro straps will be applied to stabilise the pelvis (applied over both anterior superior iliac spines and attached to the bed) and the thorax/spine (applied to the chest below the breasts and attached to the bed). Hips will be positioned in neutral extension and rotation, hip abduction in mid range and knee in extension. The dynamometer will be held perpendicular to the thigh above the lateral femoral condyl of the leg being tested with one foot of the tester against a wall for stability. Subjects will be instructed to push with maximum effort. One practice trial will be carried out prior to testing. A four second hip abduction isometric maximal contraction will be recorded. Mean maximum force over 3 measurements for each leg will be calculated. [49]

2. Subjects will rest for five minutes after which a Maximun Voluntary EMG Contraction (MVC) of the non-hemiplegic gluteus maximus and gluteus medius will be measured. The skin will be prepared for surface EMG placement. Skin will be shaved over the appropriate muscles and cleaned with alcohol. Surface EMG electrodes (Kendall, Tyco/Healthcare) will be placed onto the skin overlying these muscles following the "European Recommendations for Surface Electromyography" (SENIAM) guidelines with a spacing of $20 \mathrm{~mm}$.[50] The ground electrode will be placed on the clean shaven skin overlying the sacrum. EMG is measured at a sampling rate of 2048 $\mathrm{Hz}$ without filtering so that the signals are measured including DC. The digitalised data will be high pass filtered with a fourth order filter with a cut off frequency of $10 \mathrm{~Hz}$ and full wave rectified. To test gluteus maximus subjects will lie prone on an examination plinth. With hip and knee extended manual resistance will be given at the ankle joit in the opposite direction to movement. Subjects will be asked to slowly increase the force, reaching maximum effort after 3-5 seconds, hold it for 3 seconds and relax over 3 seconds. A rest for 1 minute will provided after which the test will be carried out once more. The maximum value from both tests will be used a s MVC.

To test gluteus medius, subjects will lie on the hemiplegic side. <the subject will beinstucted to lift leg leg sideways and manual resistance will be applied at the ankle in the opposite direction to movement. . Subjects will be asked to slowly increase the force, reaching maximum effort after 3-5 seconds, hold it for 3 seconds and relax over 3 seconds. A rest for 1 minute will provided after which the test will be carried out once more. The maximum value from both tests will be used a s MVC.

3. Electrodes will be removed from the non-hemiplegic side and will be applied with the same procedure as described above to the hemiplegic muscles of gluteus maximus, gluteus medius, vastus lateralis, semitendinosis, gastrocnemius and tibialis anterior. Henna will be applied to ensure accurate replacement at subsequent sessions. 
4. The electrogomiometer, foot switches and SwayStar instrumentation will also be fitted. A biaxial electrogoniometer will be placed over the anterior hemiplegic hip joint line, proximal arm in line with the anterior superior iliac spines, distal in line with the axis of the femur. Foot switches will be placed under the calcaneus and the third distal phalange of each foot to measure "initial contact" and "foot off "simultaneously with the EMG. SwayStar will be strapped to the patients waist.

5. Patients will complete the "Timed up and go test". Time taken will be recorded, EMG activity, dynamic balance and hip kinematics will be collected and video recordings will be taken.

6. Subjects will then rest for five minutes after which they will walk for twelve gait cycles three times. Subjects will rest for five minutes between each set of twelve gait cycles. Data collected during the first two and last two gait cycles will be excluded. Data from the three sets of eight remaining gait cycles will be used to calculate mean values.

7. Patients will rest for ten minutes and then walk over a set of four low (24cm high) barriers placed $1 \mathrm{~m}$ apart.

8. Measurement equipment will be removed, patients will rest for 20 minutes with a drink after which the SIS will be completed.

\section{Analysis}

Hemiplegic peak EMG values of gluteus maximus and medius will be compared as a percentage of the maximum voluntary contraction peak EMG value of the same muscles of the unaffected leg.

Activation patterns of gluteus maximus, gluteus medius, vastus lateralis, semitendinosis, gastrocnemius and tibialis anterior will be assessed at baseline and at subsequent data collection points. On and off times for each muscle during each stride will be calculated. An amplitude of two standard deviations higher that the resting (reference) amplitude will be considered "on" activity. All detected on- and off- times will be normalised in time using the stride time from the related heel strike measured by a foot switch. SEMG will be rectified and filtered with a high pass fourth order filter with a cut off of $10 \mathrm{~Hz}$ and plotted with the timing information along the x-axis. Total burst duration (gait cycle time minus off time) and median on and off times in percentage of the gait cycle will be calculated for each subject for each muscle. Activation patterns will be compared to established normal patterns.

For kinematic measures mean hip range of movement during 1 gait cycle in the frontal plane (ab/aduction) and in the sagittal plane (flex /ext) will be measured for the intervention groups and compared with normal values for matched controls.

Balance control will be assessed with four measurements consisting of trunk pitch (forwards-backwards) angular displacement and velocity and roll (side to side) angular displacement and velocity. Measurements will be taken during all tasks. The fibreoptic 
gyroscopes (SwayStar) will be attached via a belt to the subjects so that the sensors are at the level $\mathrm{L} 2 / 3$. The sensors will be attached to a computer via a Bluetooth communication, which will sample the velocity signals every $100 \mathrm{~ms}$ and numerically integrate the velocity signals to yield angular displacement. The mean will be taken from the maximum values of the two angular displacement measures and the two angular velocities for the cane and TheraTogs intervention groups. The Sway Star has been has been used to assess static and dynamic balance in healthy individuals of differing ages $[51,44]$ and for institutionalised older individuals [45] The means obtained for the intervention groups post treatment will therefore be compared to baseline values and to established normal values.

\section{Accelerometer Activity Monitoring}

The accelerometer (Aipermon ${ }^{\circledR} \mathrm{GmbH}$, Germany) will be attached to the patient's belt and positioned above the left hip. Patients will wear the device during waking hours during intervention time. The accelerometer will be attached after dressing in the morning and only taken off for showering, bathing and sleeping. In the statistical analysis a day starts at 24.00 o'clock and ends at 23.59 o'clock the same day. Mean activity per day will be calculated. All device settings (date, time, weight, age and gender) are pre-programmed for each patient upon receiving it and the device is switched on throughout the entire measurement period to keep patient handling of the accelerometer to a minimum. Upon completion of the intervention, the data is copied onto a PC, and its contents are viewed via ActiCoach MPAT2Viewer, Aipermon. Wearing-time include min/day spent passively (PAS: sitting), actively (ACT: movement, but not walking), walking (WLK: $0-5 \mathrm{~km} / \mathrm{h})$ ) and fast walking (FWLK: $>5 \mathrm{~km} / \mathrm{h}$ ). These are computed and analyzed. Walking speeds from $0-80 \mathrm{~m} / \mathrm{min}$ are detected as Walking and walking speeds from $83-115 \mathrm{~m} / \mathrm{min}$ are detected as Fast Walking. Speeds above $115 \mathrm{~m} / \mathrm{min}$ are considered Sportive at which point walking would turn into jogging in most individuals. Non-wearing time is indicated by the device as "resting mode". Walking and fast walking times are added to a total walking time (TWT). Activity modes and accelerometer detection accuracy have been extensively validated [46]

\section{Blinding}

All testing procedures will be carried out by dedicated trained, blinded assessors. A further research assistant will be responsible for data input. The data will be analysed by the main author. 


\section{Sample size and power calculation}

In order to detect a clinically significant difference in the primary outcome measure of "Timed up and go" of 10 seconds (from a likely range of 10-40 seconds), with a probability of $80 \%$ at a two sided 5\% significance level a total of 116 patients (58 per group) must enter the study. This calculation was performed assuming a SD of the timed up and go of $19 \mathrm{sec}$ [52] Since there are indications that the distributions of the primary endpoint variable is skewed, the use of a nonparametric test should increase the actual power. To allow for drop-outs 60 participants per intervention will be recruited.

\section{Randomization and allocation concealment}

Subjects will be randomised into cane or TheraTogs group using a computer generated randomisation programme. Allocation will be concealed with group allocation contained in centrally held sealed envelopes at Maastricht University.

\section{RESULTS}

\section{Statistical methods}

The primary analysis will be performed in an intention-to-treat fashion, i.e. all subjects who where randomised and have at least a Timed Up and Go assessment before start of therapy will be included in all analyses. In the primary analysis, missing values will be replaced by the last available value. If no value under or after treatment is available, the value measured before start of therapy will be used.

The primary analysis will be performed on the Timed Up and Go after 5 weeks of therapy. A nonparametric two sample Wilcoxon-Mann-Whitney test will be performed. Significance levels will be $5 \%$. As robustness analyses, an analysis of covariance will be performed with the value before start of therapy as covariate and centre and therapy as factors. 95\% Cl's will be calculated based on this analysis. Descriptive statistics for all data will be presented. For continuous data, this will include the change from pretherapy values. Pre-therapy values will be compared between groups to identify relevant co-variables.

\section{DISCUSSION}

Various forms of walking aids are commonly used in post stroke rehabilitation although the long term effects have been sparsely researched. In order to ensure the positive 
effect of these treatments and to enable evidence based practice, these interventions should be clinically researched.

Early walking is an important aim of stroke rehabilitation for many reasons including the psychological well-being of the patient, to prevent loss of cardiovascular fitness or the development of secondary musculoskeletal problems. This study questions whether canes are the optimal walking aid to enable early independent walking as their use inhibits rather than stimulates activity of the hemiplegic musculature and reduces the use of automatic balance responses contrary to the aims of rehabilitation. A possible alternative to cane use was recently tested by the authors in which the immediate effects of TheraTogs were to significantly improve hip abductor muscle activity compared to cane walking or walking without walking aids. [38]

The rate of recovery of all impairments after stroke is greatest in the first few weeks and slows down after two to three months. Kwakkel et al. discussed an early window during which rehabilitation has the most long term impact. [17] The potential negative or positive effects of walking aids may therefore be particularly significant in this early stage. To our knowledge no studies have investigated this questionbefore. The results of this study may have important clinical significance and could be used in the development of guidelines for gait rehabilitation following stroke.

\section{COMPETING INTERESTS}

The authors declare that they have no competing interests. 


\section{REFERENCES}

1. Sarti C, Rastenyte D, Cepaitis Z, Tuomilehto J: International trends in mortality from stroke, 1968 to 1994. Stroke 2000, 31(7):1588-1601.

2. Geschwindner HM, Rettke H, van den Heuvel WJ, Halfens RJ, Dassen T: Rehabilitation in acute stroke patients in German-speaking Switzerland. Swiss Med Wkly 2007, 137(13-14):205-211.

3. van de Port IG, Kwakkel G, van Wijk I, Lindeman E: Susceptibility to deterioration of mobility long-term after stroke: a prospective cohort study. Stroke 2006, 37(1):167-171.

4. Kollen B, van de Port I, Lindeman E, Twisk J, Kwakkel G: Predicting improvement in gait after stroke: a longitudinal prospective study. Stroke 2005, 36(12):2676-2680.

5. Indredavik B, Rohweder G, Naalsund E, Lydersen S: Medical complications in a comprehensive stroke unit and an early supported discharge service. Stroke 2008, 39(2):414-420.

6. Feigin VL, Lawes CM, Bennett DA, Barker-Collo SL, Parag V: Worldwide stroke incidence and early case fatality reported in 56 population-based studies: a systematic review. Lancet Neurol 2009, 8(4):355-369.

7. Langhorne P, Duncan P: Does the organization of postacute stroke care really matter? Stroke 2001, 32(1):268-274.

8. Collaborative systematic review of the randomised trials of organised inpatient (stroke unit) care after stroke. Stroke Unit Trialists' Collaboration. BMJ 1997, 314(7088):1151-1159.

9. Nudo RJ: Adaptive plasticity in motor cortex: implications for rehabilitation after brain injury. J Rehabil Med 2003(41 Suppl):7-10.

10. Cheeran B, Cohen L, Dobkin B, Ford G, Greenwood R, Howard D, Husain M, Macleod M, Nudo R, Rothwell $\mathrm{J}$ et al: The future of restorative neurosciences in stroke: driving the translational research pipeline from basic science to rehabilitation of people after stroke. Neurorehabil Neural Repair 2009, 23(2):97-107.

11. Huang VS, Krakauer JW: Robotic neurorehabilitation: a computational motor learning perspective. J Neuroeng Rehabil 2009, 6:5.

12. Ottenbacher KJ, Jannell S: The results of clinical trials in stroke rehabilitation research. Arch Neurol 1993, 50(1):37-44.

13. Langhorne P, Coupar F, Pollock A: Motor recovery after stroke: a systematic review. Lancet Neurol 2009, $8(8): 741-754$

14. Buurke JH, Hermens HJ, Erren-Wolters CV, Nene AV: The effect of walking aids on muscle activation patterns during walking in stroke patients. Gait Posture 2005, 22(2):164-170.

15. Kim CM, Eng JJ: Magnitude and pattern of 3D kinematic and kinetic gait profiles in persons with stroke: relationship to walking speed. Gait Posture 2004, 20(2):140-146.

16. Kautz SA, Duncan PW, Perera S, Neptune RR, Studenski SA: Coordination of hemiparetic locomotion after stroke rehabilitation. Neurorehabil Neural Repair 2005, 19(3):250-258.

17. Kwakkel G, Kollen B, Lindeman E: Understanding the pattern of functional recovery after stroke: facts and theories. Restor Neurol Neurosci 2004, 22(3-5):281-299.

18. Rossini PM, Altamura C, Ferreri F, Melgari JM, Tecchio F, Tombini M, Pasqualetti P, Vernieri F: Neuroimaging experimental studies on brain plasticity in recovery from stroke. Eura Medicophys 2007, 43(2):241-254.

19. Johansson BB: Brain plasticity and stroke rehabilitation. The Willis lecture. Stroke 2000, 31(1):223-230.

20. Rossini PM, Pauri F: Neuromagnetic integrated methods tracking human brain mechanisms of sensorimotor areas 'plastic' reorganisation. Brain Res Brain Res Rev 2000, 33(2-3):131-154.

21. Richards LG, Stewart KC, Woodbury ML, Senesac C, Cauraugh JH: Movement-dependent stroke recovery: a systematic review and meta-analysis of TMS and fMRI evidence. Neuropsychologia 2008, 46(1):3-11.

22. Nudo RJ: Postinfarct cortical plasticity and behavioral recovery. Stroke 2007, 38(2 Suppl):840-845.

23. Nudo RJ: Mechanisms for recovery of motor function following cortical damage. Curr Opin Neurobiol 2006, 16(6):638-644.

24. Ward NS, Cohen LG: Mechanisms underlying recovery of motor function after stroke. Arch Neurol 2004, 61(12):1844-1848. 
25. Carey JR, Kimberley TJ, Lewis SM, Auerbach EJ, Dorsey L, Rundquist P, Ugurbil K: Analysis of fMRI and finger tracking training in subjects with chronic stroke. Brain 2002, 125(Pt 4):773-788.

26. Loubinoux I, Carel C, Pariente J, Dechaumont S, Albucher JF, Marque P, Manelfe C, Chollet F: Correlation between cerebral reorganization and motor recovery after subcortical infarcts. Neuroimage 2003, 20(4):2166-2180.

27. Enzinger C, Johansen-Berg H, Dawes H, Bogdanovic M, Collett J, Guy C, Ropele S, Kischka U, Wade D, Fazekas $\mathrm{F}$ et al: Functional MRI correlates of lower limb function in stroke victims with gait impairment. Stroke 2008, 39(5):1507-1513.

28. Nudo RJ, Milliken GW, Jenkins WM, Merzenich MM: Use-dependent alterations of movement representations in primary motor cortex of adult squirrel monkeys. J Neurosci 1996, 16(2):785-807.

29. Wolf SL, Winstein CJ, Miller JP, Taub E, Uswatte G, Morris D, Giuliani C, Light KE, Nichols-Larsen D, Investigators $\mathrm{E}$ : Effect of constraint-induced movement therapy on upper extremity function 3 to 9 months after stroke: the EXCITE randomized clinical trial. JAMA 2006, 296(17):2095-2104.

30. Hakkennes S, Keating JL: Constraint-induced movement therapy following stroke: a systematic review of randomised controlled trials. Aust J Physiother 2005, 51(4):221-231.

31. Ajemian S, Thon D, Clare P, Kaul L, Zernicke RF, Loitz-Ramage B: Cane-assisted gait biomechanics and electromyography after total hip arthroplasty. Arch Phys Med Rehabil 2004, 85(12):1966-1971.

32. Neumann DA: Hip abductor muscle activity as subjects with hip prostheses walk with different methods of using a cane. Phys Ther 1998, 78(5):490-501.

33. Neumann DA: An electromyographic study of the hip abductor muscles as subjects with a hip prosthesis walked with different methods of using a cane and carrying a load. Phys Ther 1999, 79(12):1163-1173; discussion 1174-1166.

34. Maki BE, Mcllroy WE: Cognitive demands and cortical control of human balance-recovery reactions. J Neural Transm 2007, 114(10):1279-1296.

35. Mochizuki G, Sibley KM, Cheung HJ, Camilleri JM, Mcllroy WE: Generalizability of perturbation-evoked cortical potentials: Independence from sensory, motor and overall postural state. Neurosci Lett 2009, 451(1):40-44.

36. Woollacott M, Shumway-Cook A: Attention and the control of posture and gait: a review of an emerging area of research. Gait Posture 2002, 16(1):1-14.

37. Hamzat TK, Kobiri A: Effects of walking with a cane on balance and social participation among community-dwelling post-stroke individuals. Eur J Phys Rehabil Med 2008, 44(2):121-126.

38. Maguire C, Sieben JM, Frank M, Romkes J: Hip abductor control in walking following stroke -- the immediate effect of canes, taping and TheraTogs on gait. Clin Rehabil 2010, 24(1):37-45.

39. Holden MK, Gill KM, Magliozzi MR, Nathan J, Piehl-Baker L: Clinical gait assessment in the neurologically impaired. Reliability and meaningfulness. Phys Ther 1984, 64(1):35-40.

40. Folstein MF, Folstein SE, McHugh PR: "Mini-mental state". A practical method for grading the cognitive state of patients for the clinician. J Psychiatr Res 1975, 12(3):189-198.

41. Ng SS, Hui-Chan CW: The timed up \& go test: its reliability and association with lower-limb impairments and locomotor capacities in people with chronic stroke. Arch Phys Med Rehabil 2005, 86(8):1641-1647.

42. Andersson AG, Kamwendo K, Seiger A, Appelros P: How to identify potential fallers in a stroke unit: validity indexes of 4 test methods. J Rehabil Med 2006, 38(3):186-191.

43. Heung TH, Ng SS: Effect of seat height and turning direction on the timed up and go test scores of people after stroke. J Rehabil Med 2009, 41(9):719-722.

44. Allum JH, Carpenter MG: A speedy solution for balance and gait analysis: angular velocity measured at the centre of body mass. Curr Opin Neurol 2005, 18(1):15-21.

45. Bischoff-Ferrari HA, Conzelmann M, Stähelin HB, Dick W, Carpenter MG, Adkin AL, Theiler R, Pfeifer M, Allum JH: Is fall prevention by vitamin $\mathrm{D}$ mediated by a change in postural or dynamic balance? Osteoporos Int 2006, 17(5):656-663.

46. Jehn M, Schmidt-Trucksäess A, Schuster T, Hanssen H, Weis M, Halle M, Koehler F: Accelerometer-based quantification of 6-minute walk test performance in patients with chronic heart failure: applicability in telemedicine. J Card Fail 2009, 15(4):334-340. 
47. Duncan PW, Wallace D, Lai SM, Johnson D, Embretson S, Laster LJ: The stroke impact scale version 2.0. Evaluation of reliability, validity, and sensitivity to change. Stroke 1999, 30(10):2131-2140.

48. Lai SM, Perera S, Duncan PW, Bode R: Physical and social functioning after stroke: comparison of the Stroke Impact Scale and Short Form-36. Stroke 2003, 34(2):488-493.

49. Click Fenter P, Bellew JW, Pitts TA, Kay RE: Reliability of stabilised commercial dynamometers for measuring hip abduction strength: a pilot study. Br J Sports Med 2003, 37(4):331-334.

50. Hermens HJ, Freriks B, Merletti R et al. European recommendations for surface electromyography (SENIAM). CD-rom Enschede, Roessingh Research and Development, 1999

51. Allum JH, Zamani F, Adkin AL, Ernst A: Differences between trunk sway characteristics on a foam support surface and on the Equitest ankle-sway-referenced support surface. Gait Posture 2002, 16(3):264-270.

52. Walker C, Brouwer BJ, Culham EG: Use of visual feedback in retraining balance following acute stroke. Phys Ther 2000, 80(9):886-895. 



\title{
Chapter
}

\begin{abstract}
An orthotic garment improves gait and balance when replacing canes in chronic, previously cane using stroke-patients. A replicated, single case experimental study.
\end{abstract}

Clare Maguire, Judith M. Sieben, Nathanael Lutz, Gisela van der Wijden, Heike Scheidhauer, Robert. A. de Bie 


\section{ABSTRACT}

Background Hip-joint and leg-muscle loading facilitates normal Central-PatternGenerator activity and high hemiplegic muscle-activity increases ipsilesional corticalplasticity and improves function. Cane-walking post-stroke typically unloads hemiplegichip and muscles and reduces muscle-activity. An orthotic-garment TheraTogs allows normal joint-loading and increases muscle-activity.

Objective To investigate the effect of TheraTogs on gait and balance when replacing canes in chronic stroke-patients.

Methods Replicated, single-case ABC experiment with 4 subjects. Weekly outcome assessment of Functional-Gait-Assessment(FGA) and trunk-sway in walking. Randomized phase-duration: PhaseA Usual cane-walking for 9-12 weeks, PhaseB TheraTogs worn throughout the day and cane reduction for 9-19 weeks and PhaseC Follow-up on either no walking-aid, TheraTogs or Cane for 9-10 weeks

Results FGA score), p-value(Friedmans-Anova) and phaseC walking-aid.

Subject 1: A10, B12.5, C12.5 p=0.05, C=reduced cane-use.

Subject 2: $A 13, B 15.5, C 20 * p=0.001, C=$ no walking-aids.

Subject 3: A12, B14.5, C11 $p=0.02, C=$ cane-use.

Subject 4: $A 8, B 14 *, C 12$ p=0.002 C=intermittent TheraTogs \& reduced cane-use.

Trunk-sway measured as Total-Angle-Area $\left(T A A^{\circ}\right)$ in the frontal and sagittal-planes, reduced in two patients from phase $A$ to $B$ and in three patients from $A$ to $C$ indicating increased stability. Improvements from A to B were maintained or continued in all subjects in phaseC although no TAA changes were statistically significant.

Conclusions One patient became fully independent. Others improved but effect-sizes were smaller, thus indicating that walking-aids decisively impact gait and balanceability. Walking-aids which increase muscle-activity, that allow normal joint-loading and do not require hands may be more effective than canes. Further research is needed to confirm these results and identify which patients benefit.

Keywords cerebrovascular stroke, canes, orthoses, walking, postural balance 


\section{INTRODUCTION}

Stroke is the second leading cause of acquired disability in adults and stroke survivors worldwide number 33 million.[1] Disability-Adjusted-Life-Years(DALYs) lost due to stroke, are estimated at 102 million.[2] Importantly, most of the burden of stroke falls on resource-poor, low and middle income countries (70.9\% stroke deaths, and $77.7 \%$ DALYs lost).[1]

Of those patients who survive stroke, approximately 65\% are left with some form of disability.[3] At 6 months 50\% have hemiparesis, 30\% are unable to walk without assistance, $26 \%$ are dependent in activities of daily living, $26 \%$ are institutionalized and $35 \%$ have depressive symptoms.[4] Taken together, these figures emphasise the need for more effective and affordable rehabilitation interventions.

As well as developing novel, often expensive treatments such as robotic and treadmill training $[5,6]$ current, widely used interventions, particularly those which are inexpensive, should be critically evaluated in order to optimize prescription and application. Walking-aids, most commonly canes, used in post-stroke rehabilitation have primarily been investigated for the immediate effects on kinematic and kinetic parameters in cross-sectional studies.[7-9] These studies widely conclude that walking-aids positively influence gait and broadly recommend use in clinical practice. Indeed a recent clinical practice guideline stated "in the opinion of the guideline development team, the use of walking-aids is beneficial to patients with a stroke in terms of safety, independence, and efficiency of walking, as well as confidence."[10] However, a number of prospective observational studies have demonstrated that stroke patients using canes long-term have lower balance scores and show less social participation than those without[11] that balance and falls efficacy are reduced in stroke patients who regularly used walking-aids,[12] and that the sensorimotor function of the non-hemiplegic, cane holding hand is significantly reduced compared to non-cane using stroke patients.[13] These results appear to support studies in the general population which indicate a high positive correlation between walking aid use and fall risk.[14]. However no causal relationship has been established through experimental studies.

The impact of walking-aids on balance and gait-function in post-stroke patients therefore remains unclear. To date no prospective studies have investigated the long term impact of walking-aids on balance and gait-function in chronic stroke patients.

\section{Disadvantages of cane use}

The basic pattern of human walking is directed by spinal Central-Pattern-Generators (CPGs).[15-18] CPG activity is influenced by descending signals from higher centers and by peripheral afferent feedback. Studies with spinal-cord injury patients demonstrate that stepping patterns can be driven solely by peripheral afferents via the CPGs.[19] Normal joint loading, particularly at the hip joint, normal muscle loading, hip joint 
movement as well as cutaneous and muscular proprioceptors of the foot and ankle, appear to be particularly influential in determining final motor output and leg muscle activation patterns.[17,18,20]. Walking with canes in the contralateral, non-hemiplegic hand unloads the opposite, hemiplegic hip [21,22], thus preventing normal hip jointload feedback to CPG circuits. Hemiplegic muscles are also unloaded and muscle activity on the hemiplegic side is reduced.[22] Afferent feedback to CPG circuits, potentially already disturbed due to stroke induced sensorimotor changes, may thus be further disrupted by cane use. CPG output may be adversely impacted.[23]

Spinal CPGs appear to be responsible for upper and lower limb coordination during movements such as running. [24,25] Humans can disengage the upper limbs from four limb control when required.[26] Spinal circuits can be "gated" via cortical activity to free arm movements. This implies that upper limb activity outside cyclical CPG control when walking, requires increased cortical input.[25,27] Hand held walking-aids such as canes require upper limb decoupling from four limb CPG control, which may demand increased cognitive resources. $[25,27]$ This may have a negative impact on patients with reduced balance, who often use more cognitive resources to maintain stability.[28]

These effects may be relevant in rehabilitation as recent animal studies indicate that plastic changes in spinal-cord circuitry occur post-stroke and impact functional recovery.[29] Sensory afferents may provide a route to influence spinal plasticity and improve post-stroke function. It has been shown in spinal injury patients that "segmental influence of spinal networks can play a significant role in appropriate functional organisation of motor output"[19] and that spinal and cortical plasticity is influenced by the pattern of sensory afferent feedback.[30]

Cortical plasticity is also use-dependent post-stroke. Ipsilesional cortical plasticity following stroke correlates with improved functional outcomes and is increased by activity on the hemiplegic side.[31] Cane-walking significantly reduces muscle activity on the hemiplegic side[22] thus potentially decreasing ipsilesional plasticity and consequently functional outcomes. Walking-aids which do not reduce or which even increase hemiplegic muscle activity may induce more beneficial neuroplastic changes.

Numerous factors which are known to impact neuroplasticity and motor-learning are present during cane use in post stroke patients. High intensity, task-specific training involving meaningful tasks, for which patients are highly motivated have the largest influence on neural plasticity and motor-learning.[32-34] As walking-aids are often used for long periods during the day causing high repetition,for the important task of walking, the influence on plasticity may be significant.

It has been shown that levels of ambulation and cardiovascular fitness are extremely low in chronic stroke survivors. These secondary consequences of stroke increase disability. Various mobility deficits, particularly poor balance and muscle-strength, contribute to low activity levels and deconditioning.[35,36] Balance has also been shown to correlate strongly to walking ability post-stroke.[36,37] Interventions which improve dynamic balance may therefore help to increase walking function and activity levels. 
Cane walking increases the base of support potentially reducing the need to use balance reactions to maintain stability. Typical "ankle" and "hip" balance strategies[38] may be biomechanically impeded by the cane, whereas untypical activity of the caneholding arm may be facilitated. Reduced hemiplegic muscle activity during cane walking may impact the recovery of muscle-strength.

We suggest therefore, that walking-aids should allow normal joint and muscle loading, have minimal effect on muscle activity and length during walking and should not require the use of hands.

\section{Orthotics as an alternative to cane use}

The orthotic garment and strapping system TheraTogs increases muscle activity during gait.[22,39] As nothing is pushed or leant upon, hands are not used and the base of support is not increased, joints and muscles remain loaded when walking. Therefore this walking device may be more effective in gait rehabilitation following stroke than canes.

\section{Research question}

The aim of this study was therefore to investigate the influence of TheraTogs on balance and walking ability when replacing canes in chronic, cane using stroke patients.

\section{METHODS}

\section{Study design}

A replicated single subject $A B C$ experimental design. $A B C$ designs are recommended in cases where interventions are expected to cause stable changes.[40]

\section{Participants}

A convenience sample of four patients was recruited from the physiotherapy outpatient department of the "REHAB" neurorehabilitation centre in Basel, Switzerland.

\section{Inclusion criteria were:}

1. Chronic stroke (> six months since last stroke). 2. Independent cane walkers. Subjects used canes to walk inside (this could be intermittent changing from cane to holding furniture etc.) and always when outside. 3. A Mini Mental Score of 22 or above.[41] 
Chapter 8

\section{Exclusion criteria were:}

1. Patients at risk of falling identified as a Berg Balance Score $\leq 42 / 56$.[42]

\section{Intervention}

The study consisted of three phases $A, B$ and $C$. Randomisation of time periods of baseline-A, intervention-B and follow-up-C, was performed to improve internal validity, potentially threatened by time related changes in the dependent variables. [40]

- PhaseA(Baseline). Subjects continued to walk unchanged with a cane for a period of 9 to 12 weeks during which period outcomes were measured weekly to establish baseline values. Participants were randomised into baseline periods of 9, 10, 11 or 12 weeks duration.

\section{TheraTogs fitting}

During this period subjects were individually fitted with TheraTogs, applied to support hemiplegic hip extensor and abductor musculature(Fig.1). The upper-and lower body parts of the orthosis were tailored to ensure comfortable neck, arm and leg fit. Elastic straps were applied to facilitate muscle activity. One end of the strap was sewn onto the garment and the second attachment position was marked to enable daily, standardised fitting. Caregivers were taught in as many sessions as necessary(2-3) how to fit and remove the orthotic garment. Written and photographic instructions were provided. TheraTogs was fitted to wear beneath undergarments to allow toileting without having to remove the orthosis.

- PhaseB (TheraTogs Intervention). Subjects were randomized into intervention periods of 9 to 16 weeks. During this period TheraTogs was applied when dressing in the morning and removed before bed at night. On measurement days, weekly measurements were conducted in the morning and TheraTogs was fitted afterwards by physiotherapy staff in the clinic. Caregivers were offered ongoing support during this period if necessary. Subjects were instructed to try to stop using the cane. If this was not possible in all situations, then to reduce cane use to the minimum and to note how often and it what circumstances it was used.

- PhaseC(Follow-up). Subjects were randomized into follow-up periods of 9-10 weeks. During this phase participants were able to choose between walking 1. with a cane 2.with TheraTogs 3. without an assistive device. 

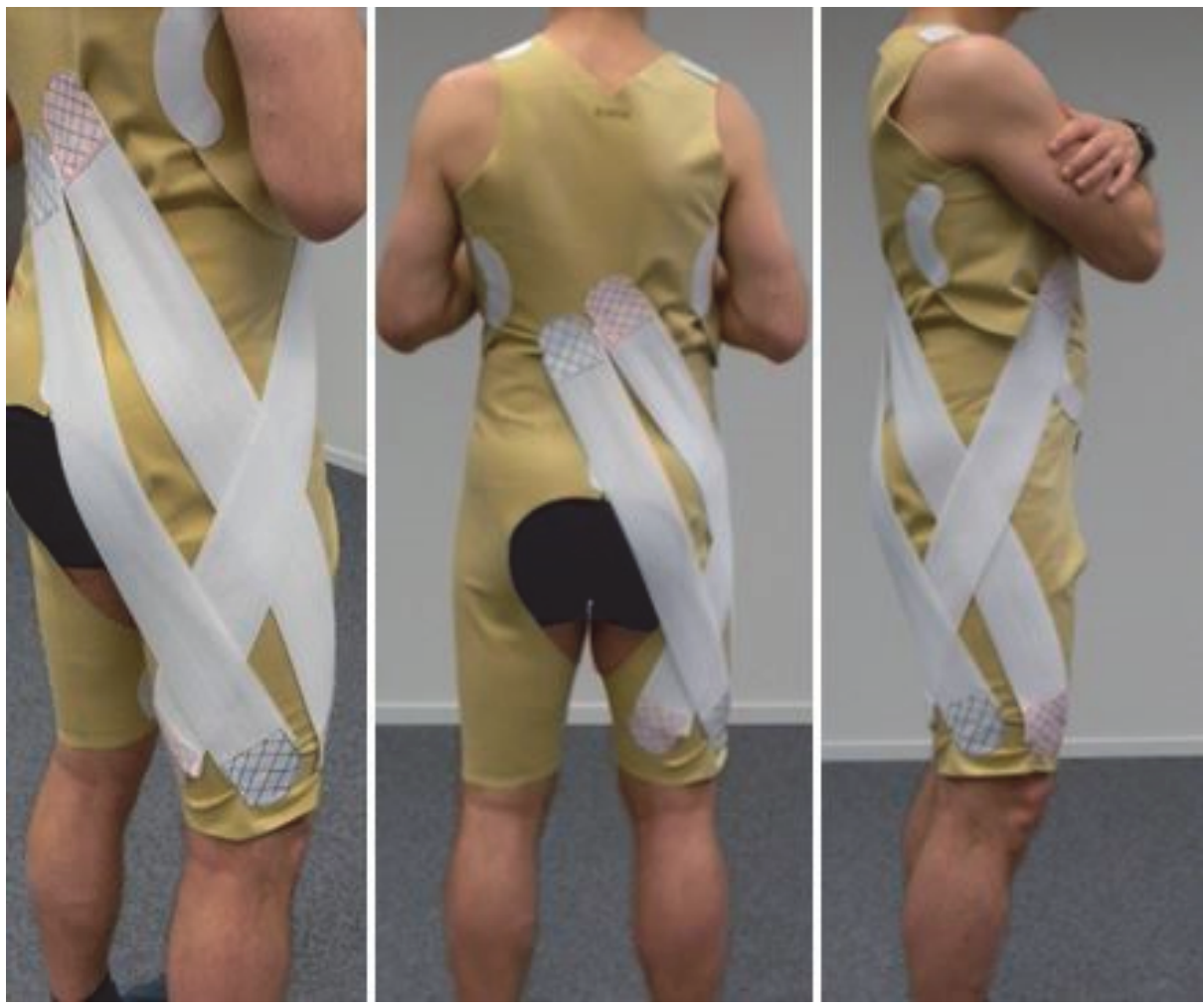

Figure 1. Standard TheraTogs application

\section{Standard Therapy}

All patients were receiving out-patient physiotherapy, occupational therapy and where necessary speech therapy once or twice weekly prior to and during the study. This continued independently of the study.

\section{Outcome Measures}

All measurements were carried out without any walking-aids, once a week during the baseline, intervention and follow-up periods.

\section{Primary Outcome}

The Functional Gait Assessment[43-45] has been shown to be a valid and reliable tool for measuring functional gait ability in stroke patients and is translated and validated for a German speaking population.[43] This test assesses postural stability during various walking tasks with 10-items, each item being scored on a scale from 0-3. This measure 
takes five to ten minutes to complete and was therefore considered practical for weekly measurements.

\section{Secondary Outcomes}

Trunk-sway measured as trunk angular displacement in the frontal and sagittal planes using two angular velocity sensors (fibreoptic gyroscopes) of the SwayStar balance system(Balance International Innovations $\mathrm{GmbH}$, Iseltwald, Switzerland).[46,47] It was worn by subjects attached via a belt at the L2/3 level. Sensors connected to PC software via a Bluetooth communication, which sampled velocity signals every $100 \mathrm{~ms}$ and numerically integrated the velocity signals to yield angular displacement. Trunk angular displacement was measured weekly during an $8 \mathrm{~m}$ walk.

Patients completed a weekly questionnaire during phaseBrecording the average number of hours per day in which TheraTogs was worn, the comfort level, continued use of a cane with or without TheraTogs as a percentage of previous (phaseA) use, the circumstances of cane use and the subjective effect on walking ability when wearing TheraTogs using a Likert scale (5 items: much worse - much better).

\section{Ethical approval}

The study was approved by the Ethics Committee of Canton Basel.

\section{Statistics}

\section{Power analysis - sample size (number of measurements per phase)}

A power analysis was conducted to determine the number of measurements necessary in each phase to detect significant differences between conditions with alpha set at 0.05 and a power (1-beta) of 0.80 .

As no studies have directly estimated a minimal clinically significant difference for the FGA in chronic stroke patients, these values were estimated based on the following information:

- Mean score of the FGA in healthy adults age 60-70 - score 27, age 70-80 - score 25.[48]

- Estimated mean of chronic stroke patients based on Lin et al. and Thieme et al. score $15[43,49]$

- The included participants will not be at risk of falling (exclusion criteria). Studies estimate a cut-off score for predicting falls with the FGA is between 15 for Parkinsons[50]) and 20-22 for community dwelling older adults.[45]

- We therefore assumed our patients would have a minimum score of 15 ranging to a maximum of 22 . We estimated a mean value of 19 . Improvement to achieve inde- 
pendent walking without a walking aid would mean reaching a score of an unimpaired elder of 25 points. This is a clinically important difference of 6 points.

Therefore $\mu(0)=19, \mu(1)=25 \sigma=7$. Based on these values sample size i.e. number of measurements necessary per phase is 9 . We determined measurements should be separated by at least one week.

\section{Statistical Analysis}

As no gold-standard for the analysis of single case data exists, it is recommended that several statistical methods are combined and compared to improve the validity of data interpretation.[51,52]

Data was plotted graphically and assessed visually for changes in level, trend and variability between baseline, intervention and follow-up phases.[53,54] The median and Inter-Quartile-Range were calculated for FGA scores in each phase. The mean and standard deviation of trunk-sway (angular excursion in the sagittal and frontal planes of the Centre of Mass) during walking in each phase was calculated. Box plots and error bars were plotted for visual analysis of differences between phases.

Several methods were used for statistical analysis, the split method of trend estimation, Cohen's d for different sized groups to estimate the effect-size between phases and Friedmans Anova and Wilcoxon ranks tests.

The split method of trend estimation - the "celeration line". This is a method commonly used to analyse single case experimental data. A trend line is calculated in the baseline phase using median values in the first and second half of that phase to determine the gradient of the slope. The gradient is maintained and the height of the slope is adjusted so that $50 \%$ of the data points fall above the line and $50 \%$ fall below. This line is then extended through the intervention phase to provide a visual guide for predicting behavior over time if baseline trends had remained unchanged. In effect, the celeration line tests the hypothesis that there is no difference in behavior across the two phases. If the intervention data points also fall such that $50 \%$ of them are above and $50 \%$ below the celeration line, then it may be concluded that there was no change in behavior resulting from introduction of the treatment.[53] If this is not the case, the percentage of observed data points above or below the line is subtracted from the baseline value to give an indication of change in trend.[55] This procedure is repeated from the intervention to the follow-up phase.

\section{RESULTS}

Four cane using, chronic stroke survivors with a mean (SD) time since stroke in years of 4.5(1.6)) reduced or stopped cane use and replaced this with a wearable orthosis, 
TheraTogs, over a period of 8 to 19 weeks (mean(SD) 12(4.7)) in phaseB. In subjects 1, 2 and 4 TheraTogs was worn seven days a week; in subject 3 , due to difficulty applying TheraTogs in the morning, this was three days a week and application was done in the REHAB clinic.

Subject 2 was randomised to a period of 14 weeks in phase $B$, however due to improvements in balance and walking ceased using TheraTogs or cane, on request, after 8 weeks. Subject 3 was randomised to a period of 13 weeks in phase B. However as he was only able to wear TheraTogs three days a week he requested to have this period extended, and continued for 19 weeks.

Although average daily wear of TheraTogs in hours was similar between subjects (mean(SD), 10(1.08)) cane use in phase B as a percentage of baseline use differed considerably (mean(SD) 19.55\%(22.68)). For further details see Table 1.

Interventions in phaseC varied and were determined by subject preference. Subject 1 stopped TheraTogs and returned to cane use at lower levels than baseline (75\%), subject 2 stopped using TheraTogs and cane and walked independently without aids, subject 3 stopped and returned to baseline conditions, subject 4 continued to use TheraTogs intermittently (1-2 times weekly) and returned to cane use as at baseline.

Patient presentations at baseline analysed according to the International Classification of Functioning Disability and Health (ICF)[56] are presented in Table 2. 
Orthotic garment, canes, gait and balance, chronic stroke

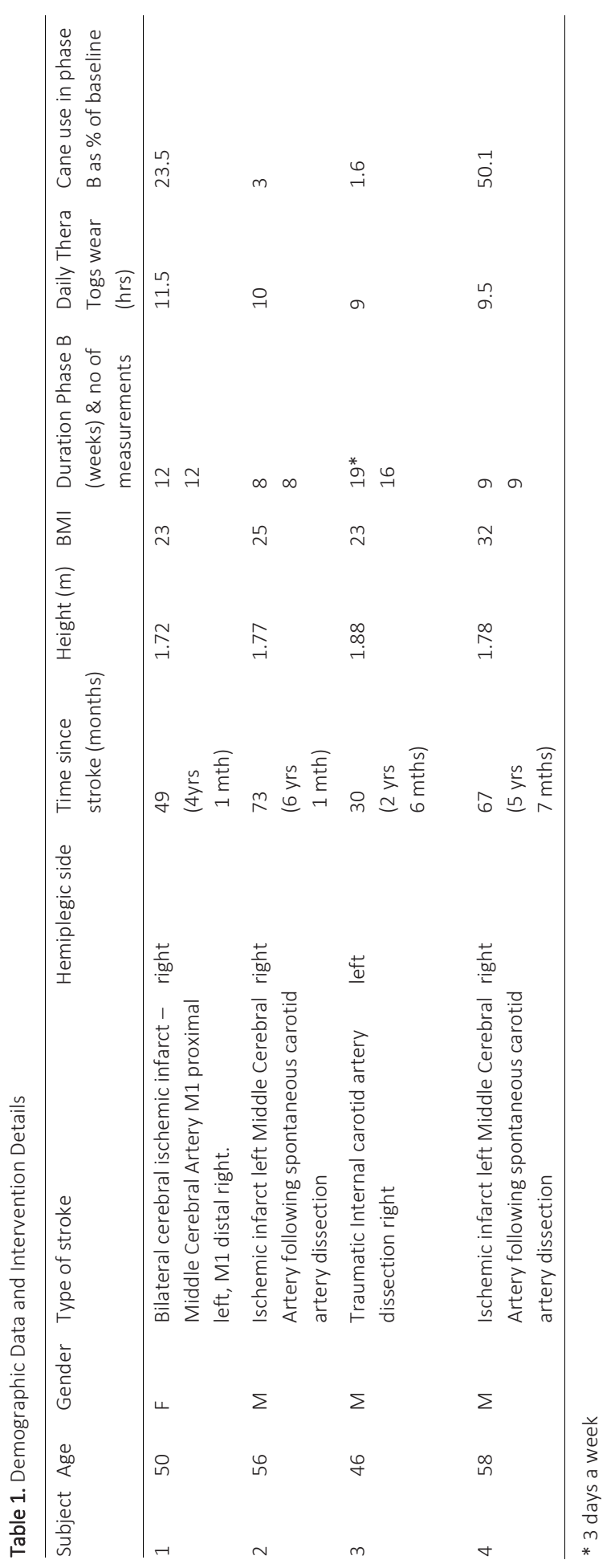




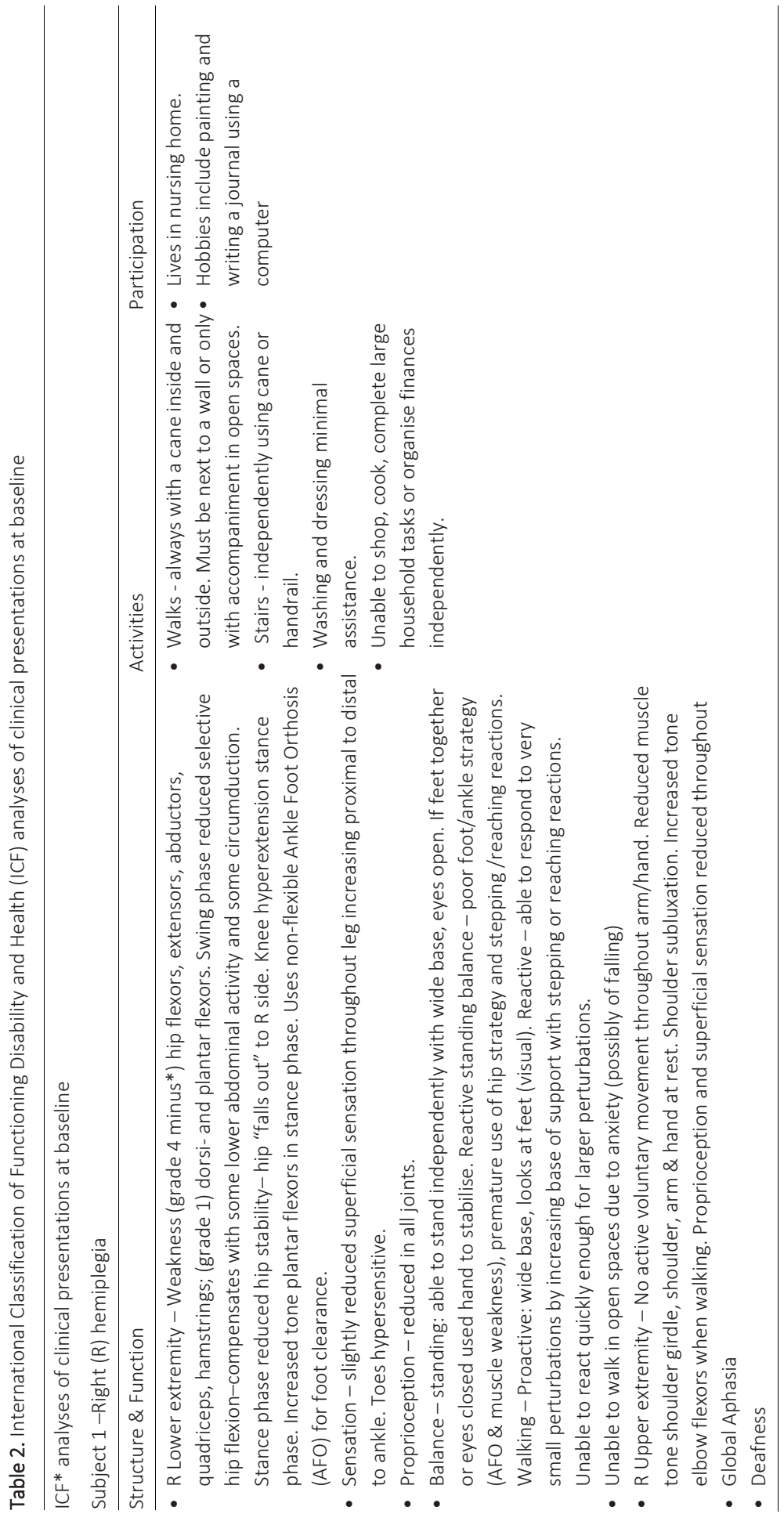


Orthotic garment, canes, gait and balance, chronic stroke

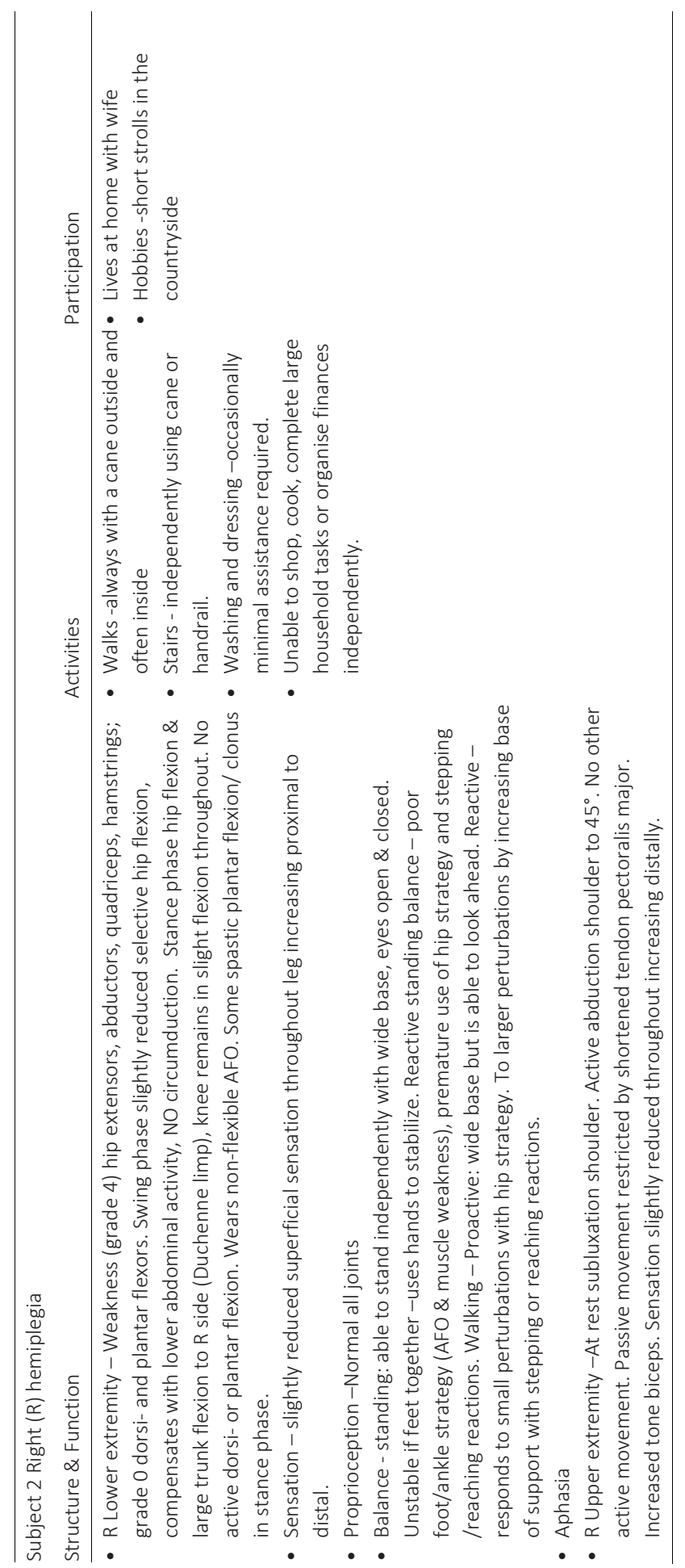




\section{Chapter 8}

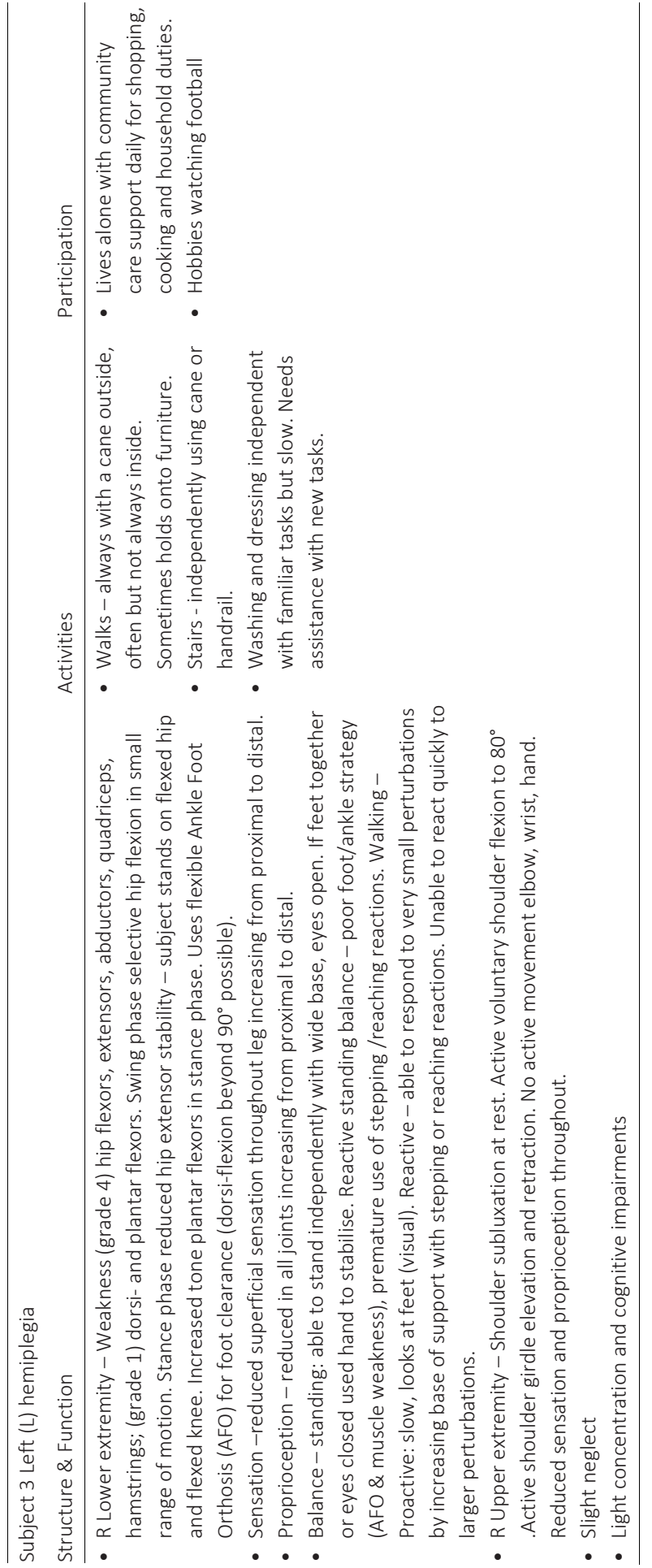


Orthotic garment, canes, gait and balance, chronic stroke

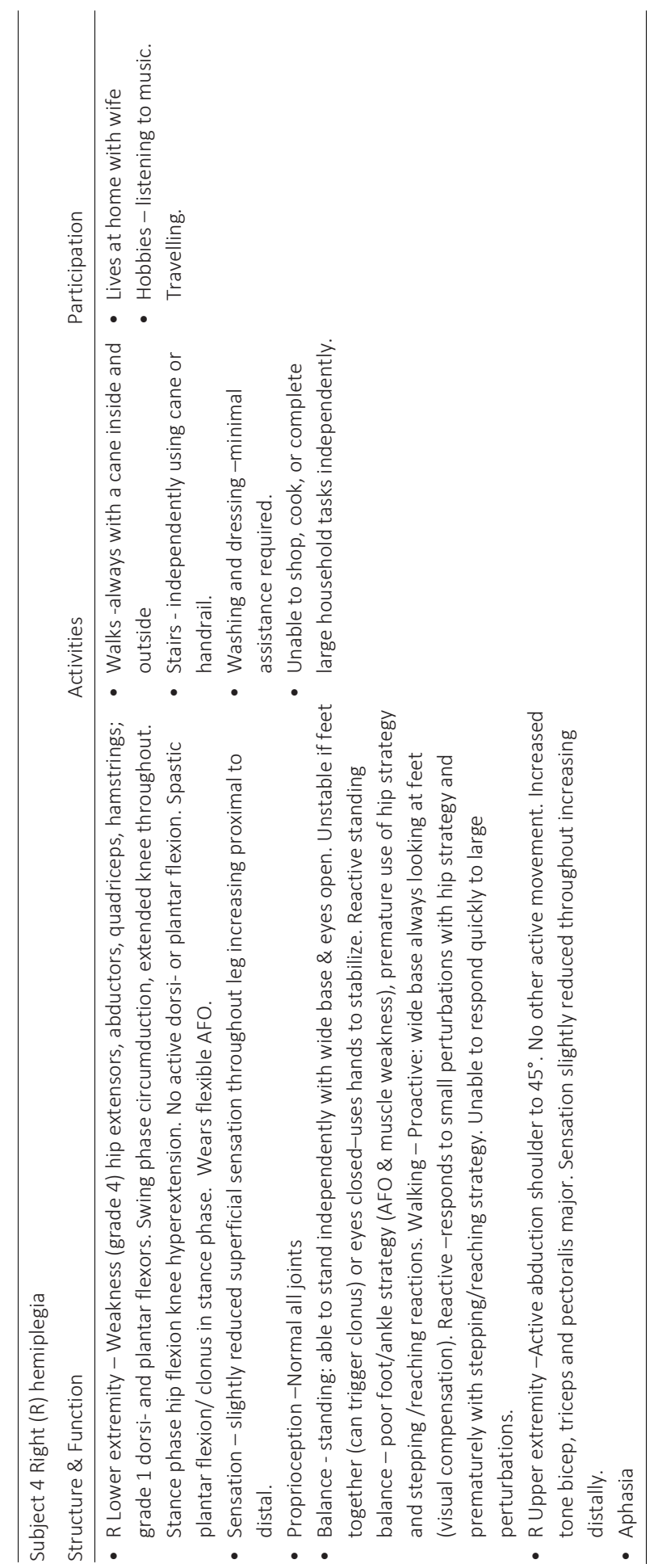


Figure 2. shows FGA and trunk-sway data plotted against time. Visual analysis demonstrates jumps in FGA values between phases $A$ and $B$ for all subjects, and reductions between phases $B$ and $C$ for all except subject 2. In subject 1, $83 \%$ of measurement points in $B$ are above the extended celeration line from $A$, indicating a $33 \%$ improvement from baseline. In subjects 2, $3 \& 4$ all points are above the line, indicating at least $50 \%$ improvement. In phaseC, $66 \%$ of measurement points from subject 1 are below the extended celeration line from $B$, indicating a $16 \%$ deterioration, subject 2 shows a $12.5 \%$ improvement from $B$ to $C$, subjects 3 and 4 show at least $50 \%$ deterioration, as all measurement points fall below the extended celeration line.

Reduced values of trunk-sway indicate balance improvement. In subject 1 trunksway measurements from A to B remained distributed evenly around the extended celebration line, indicating no change. Subjects 2 \& 4 showed a 50\%(at least) and 39\% improvement respectively. Subject 3 showed a $50 \%$ deterioration. From phase B to C subject 1 showed a 17\% deterioration, subject 2 a 17.5\% improvement, subjects 3 and 4 a deterioration of at least $50 \%$.

All subjects showed significant improvements in FGA scores from A(baseline) to $B$ (intervention) (see Table 3). Subject 4 scores showed an improvement exceeding the Minimal-Clinical-Important-Difference(MCID) between these phases; effect-sizes were large in all subjects. Scores from B to $C$ deteriorated slightly but not significantly in subject 1 , with a small negative(non-significant) effect-size; improved significantly in subject 2 with a large effect-size; change from phase A to C exceeded the MCID; deteriorated significantly in subjects 3 and 4 with medium to large (although non-significant) effectsizes. The phaseC scores of subjects $1 \& 4$ nevertheless remained higher than baseline.

Trunk-sway improved (reduced) in subjects 1, 2 and 4 from phase A to phaseB although changes were not significant. Effect-sizes were small to medium but not significant. Trunk-sway increased non-significantly from A to B in subject 3 with a large but non-significant effect-size. Although visual analysis showed a deteriorating trend in trunk-sway values from B to C, mean values showed a small but non-significant improvement. Effect-sizes were small to medium and also non-significant.

Figure 3 shows results of subjects' weekly questionnaire regarding TheraTogs and cane use. Results indicate that TheraTogs was considered comfortable, helped walking and was consistently worn for most of the day. Cane reduction was more varied as indicated earlier. 

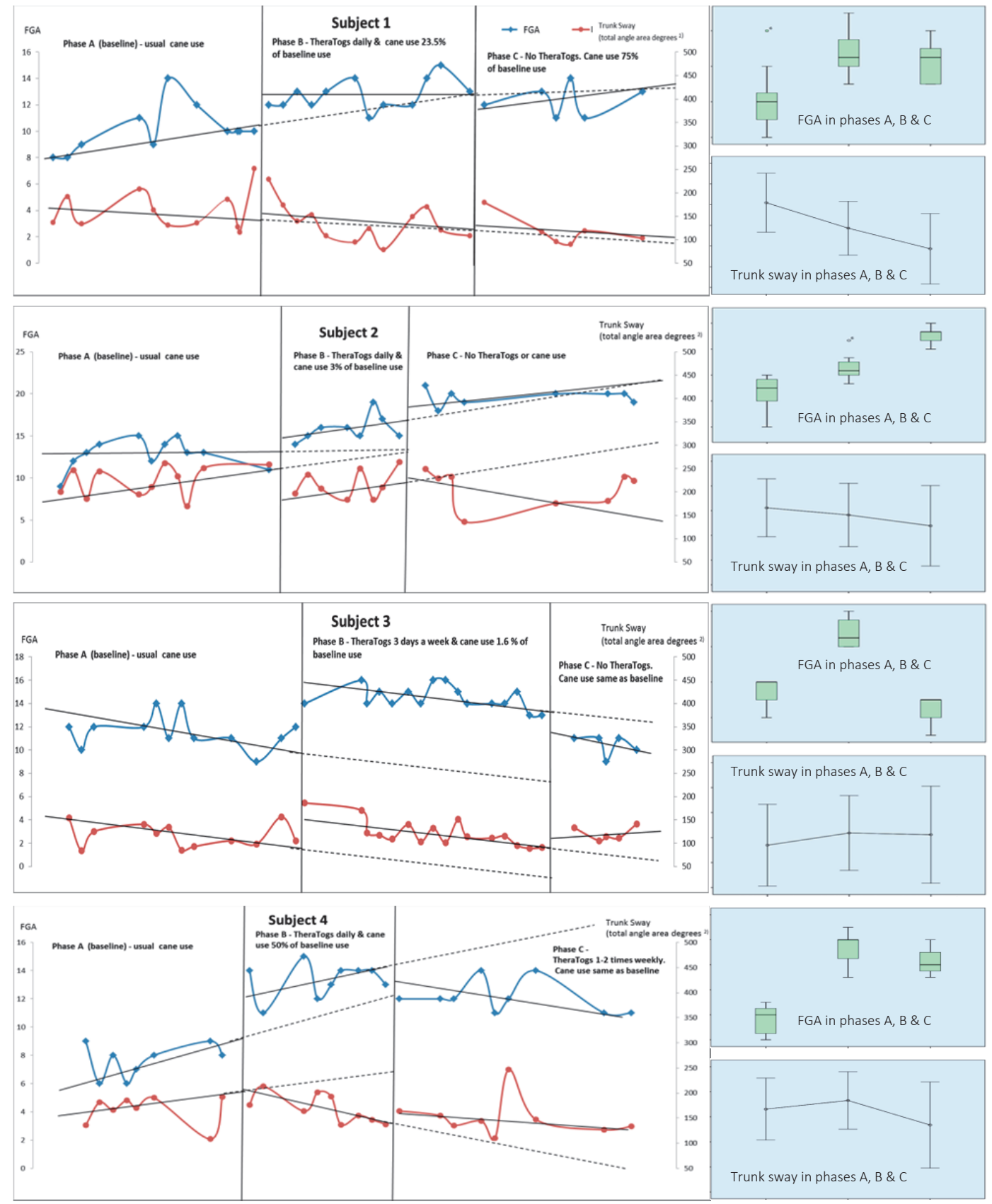

Time in weeks

Figure 2. Functional Gait Assessment (FGA) and Trunk-sway plotted against time showing celebration lines for each phase for each subject with FGA box-plots and Trunk-sway error bars 


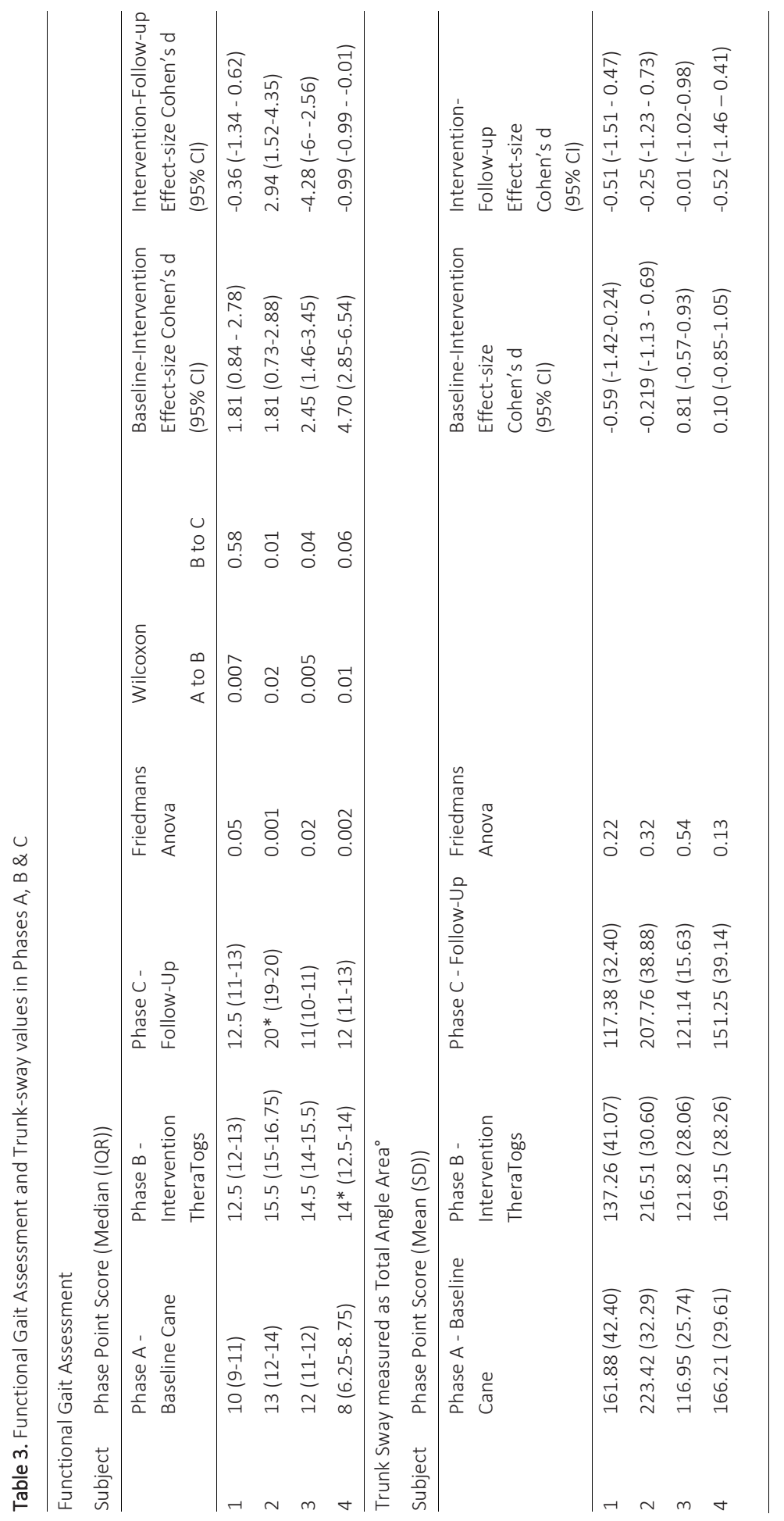


Orthotic garment, canes, gait and balance, chronic stroke

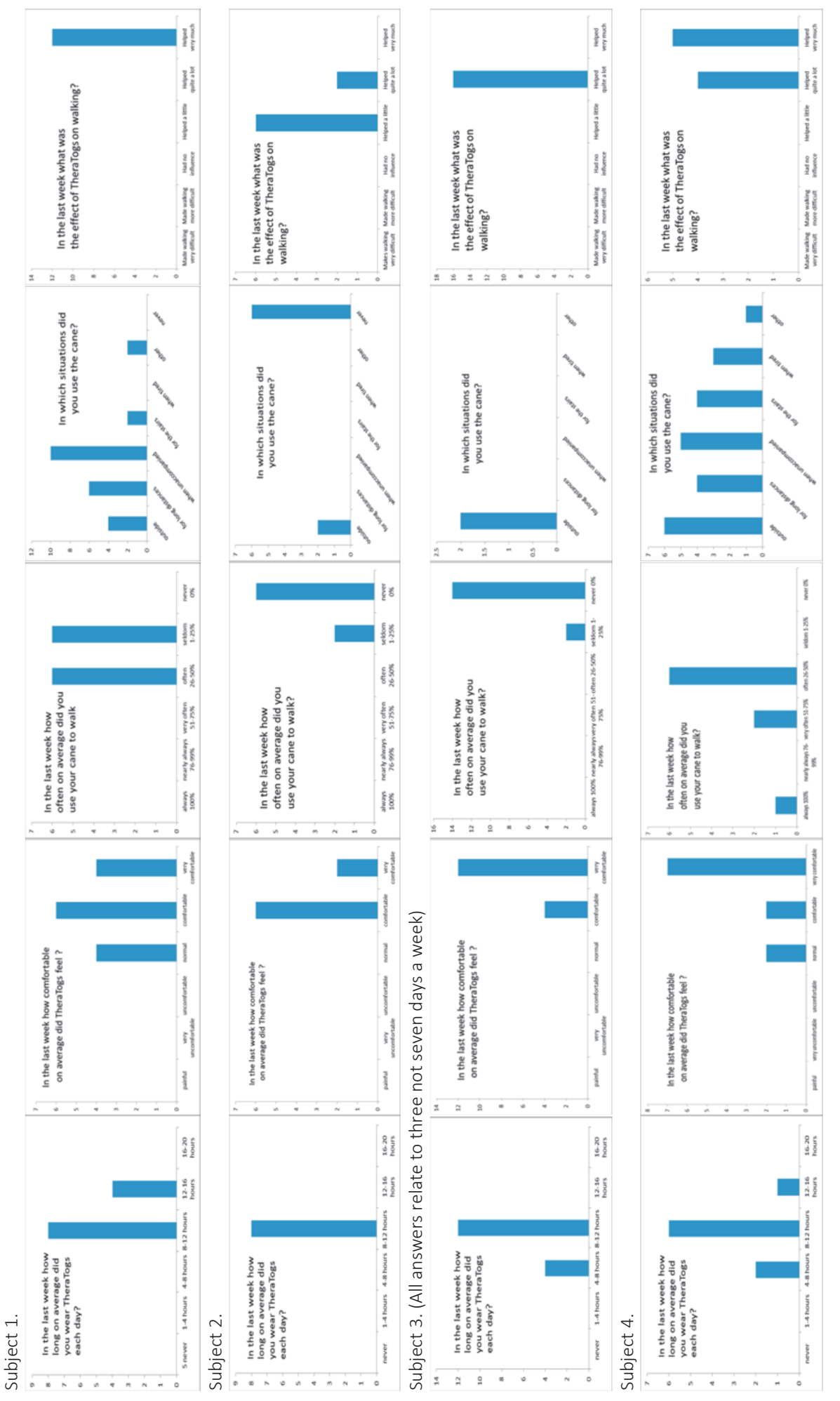

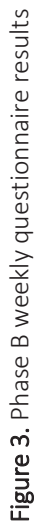




\section{DISCUSSION}

In this replicated single case study we examined the effect on gait and balance when reducing cane use and replacing it with an orthosis TheraTogs, in chronic, cane using stroke patients. To our knowledge, this is the first experimental, prospective study to investigate these effects in chronic stroke patients.

All subjects showed improvements in the primary outcome, FGA, between baseline phaseA and intervention phaseB. All analyses techniques confirmed this improvement.

Although reductions in cane use and clinical presentations varied, time spent wearing TheraTogs was similar between subjects. This suggests that time potentially benefitting from the increase in muscle activity facilitated by TheraTogs[22] was also similar. The long term impact of canes, which reduce muscle activity, or TheraTogs, which increase muscle activity[22], on strength has not been specifically investigated. It may be that walking-aids which increase muscle activity positively impact strength and viceversa. As increased muscle-strength is associated with improved balance and reduced fall risk[57], this may have been an important factor in outcomes.

The largest effect in FGA from A to B occurred in subject 4, who showed an improvement exceeding the MCID, despite beginning with the lowest score and reducing cane use the least (50\%). This was the only subject demonstrating pronounced clonus in the hemiplegic leg, which was easily triggered during standing and walking. As clonus appears to be very sensitive to afferent input from load receptors[58], it may be that increasing joint loading when walking without the cane was particularly relevant for this pathophysiology. Reduced clonus may allow improved weight transfer onto the hemiplegic leg and more stability in stance phase.

Subject 3, who reduced cane use almost completely when wearing TheraTogs, although only three days a week, also demonstrated a large effect-size from phase A to B. Clinically this subject demonstrated the most pronounced reduction in proprioception. More normalised afferent input to spinal circuits due to increased loading of joints and muscles without cane, may have been particularly beneficial for disrupted joint position sense.

Responses from phases $\mathrm{B}$ to $\mathrm{C}$ were more variable, possibly reflecting different interventions. Only subject 2 , whose progress in phaseB enabled him to walk without aids in phaseC, continued to improve. In this subject, afferent feedback to the hemiplegic limb remained normalised in phase $C$ as in phaseB, muscle activity was not reduced due to cane use, arms were not decoupled from four-limb CPG control and hip strategy balance reactions could be accessed. All other subjects showed some deterioration from phases B to C, although subjects 1 (who reduced cane use in phaseC to $75 \%$ of baseline values) \& 4 (who continued to intermittently use TheraTogs) maintained scores above baseline levels.

These changes indicate that gains were not completely stable and were influenced by ongoing use of walking-aids suggesting that a longer intervention period may be necessary to create established neuroplastic changes. 
Reductions in trunk-sway during walking reflect improved balance. Therefore, gains in FGA scores would be expected to negatively correlate with trunk-sway values. This was the case in subjects $1 \& 2$. However in subjects $3 \& 4$, who showed most gains in FGA from $A$ to $B$, mean values of trunk-sway in B increased slightly (although trend was negative). This may be because increasing gait speed can increase trunk-sway.[59] Increased gait speed in these subjects, measured during FGA and $8 \mathrm{~m}$ walk, may have led to higher trunk-sway values.

Variations in body weight and height lead to differing biomechanical forces, which could affect the efficacy of TheraTogs. However subjects $3 \& 4$ who showed the largest effect-sizes in primary outcome were the tallest and had the highest BMI respectively.

Fear appeared to be an important factor in the ability to reduce case use. Subjects 1 and 4 showed most anxiety when walking without a cane. Subject 1 compensated by walking near walls or furniture, subject 4 preferred to be accompanied when without cane and therefore reduced cane use the least.

Questionnaire results showed that subjects found the individualised TheraTogs comfortable and felt it stabilised gait. Caregivers reported that standardised application was quick and easy. However, despite positive feedbacks only one subject continued using the orthosis post intervention. Subject 3 was unable to apply TheraTogs independently despite special adaptation and several teaching sessions.

It remains unclear from these results how much improvement was due to TheraTogs and how much to reduced cane use. A combination of effects is likely. TheraTogs may increase muscle activity and provide stability, which in turn may enable a reduction of cane use. This would allow more normal afferent feedback, reduce the artificially increased base of support, thus requiring increased use of hip and ankle balance strategies and reduce the cognitively demanding de-coupling from four limb CPG control.

As FGA consists of a battery of tests which were repeated weekly, it is likely that some score changes were due to learning effects rather than improvement in gait-function. However, these effects most likely occurred during the first weeks of baseline testing. This would tend to overestimate improvements in baseline FGA scores and lead to an underestimation of differences between phases $A$ and $B$. These effects as well as ongoing physio and occupational-therapy may also have contributed to the unstable baseline.

This study indicates that walking-aids have a specific influence on balance and walking ability in chronic stroke subjects, and that TheraTogs use combined with cane reduction is more effective than cane use alone. Further studies are required to confirm these results and to identify which patients benefit. Clinical Practice Guidelines should be updated to reflect the results of this and other observational prospective studies regarding the effect of cane use post-stroke.

\section{CONFLICT OF INTEREST STATEMENT.}

The authors declare there is no conflict of interest. 


\section{REFERENCES}

[1] Feigin VL, Forouzanfar MH, Krishnamurthi R, Mensah GA, Connor M, Bennett DA, et al. Global and regional burden of stroke during 1990-2010: findings from the Global Burden of Disease Study 2010. Lancet. 2014 Jan 18;383(9913):245-54.

[2] Feigin VL, Mensah GA, Norrving B, Murray CJ, Roth GA. Atlas of the Global Burden of Stroke (1990-2013): The GBD 2013 Study. Neuroepidemiology. 2015;45(3):230-6.

[3] Scherbakov N, von Haehling S, Anker SD, Dirnagl U, Doehner W. Stroke induced Sarcopenia: muscle wasting and disability after stroke. Int J Cardiol. 2013 Dec 10;170(2):89-94.

[4] Go AS, Mozaffarian D, Roger VL, Benjamin EJ, Berry JD, Borden WB, et al. Heart disease and stroke statistics-2013 update: a report from the American Heart Association. Circulation. 2013 Jan 1;127(1):e6-e245.

[5] Mehrholz J, Elsner B, Werner C, Kugler J, Pohl M. Electromechanical-assisted training for walking after stroke. Cochrane Database Syst Rev. 2013;7:Cd006185.

[6] Mehrholz J, Pohl M, Elsner B. Treadmill training and body weight support for walking after stroke. Cochrane Database Syst Rev. 2014;1:Cd002840.

[7] Allet L, Leemann B, Guyen E, Murphy L, Monnin D, Herrmann FR, et al. Effect of different walking aids on walking capacity of patients with poststroke hemiparesis. Arch Phys Med Rehabil. 2009 Aug;90(8):1408-13.

[8] Cha YJ. Do traditionally recommended cane lengths equally influence walking in patients after stroke? Disabil Health J. 2015 Jan;8(1):136-9.

[9] Tyson SF. Trunk kinematics in hemiplegic gait and the effect of walking aids. Clin Rehabil. 1999 Aug;13(4):295-300.

[10] JM V, EEH vW, RPS vP, HIM H, MB R, PhJ vdW, et al. Royal Dutch Society for Physical Therapy - Clinical Practice Guideline for Physical Therapy in patients with stroke. 2014.

[11] Hamzat TK, Kobiri A. Effects of walking with a cane on balance and social participation among community-dwelling post-stroke individuals. Eur J Phys Rehabil Med. 2008 Jun;44(2):121-6.

[12] Kim O, Kim JH. Falls and Use of Assistive Devices in Stroke Patients with Hemiparesis: Association with Balance Ability and Fall Efficacy. Rehabil Nurs. 2015 Jul-Aug;40(4):267-74.

[13] Son SM, Kwon JW, Nam SH, Lee NK, Kim K, Kim CS. Adverse effects of motor-related symptoms on the ipsilateral upper limb according to long-term cane usage. NeuroRehabilitation. 2012;31(2):137-41.

[14] West BA, Bhat G, Stevens J, Bergen G. Assistive device use and mobility-related factors among adults aged>/=65years. J Safety Res. 2015 Dec;55:147-50.

[15] Nielsen JB. How we walk: central control of muscle activity during human walking. Neuroscientist. 2003 Jun;9(3):195-204.

[16] Dietz V. Neuronal plasticity after a human spinal cord injury: positive and negative effects. Exp Neurol. 2012 May;235(1):110-5.

[17] Dietz V, Müller R, Colombo G. Locomotor activity in spinal man: significance of afferent input from joint and load receptors. Brain. 2002 Dec;125(Pt 12):2626-34.

[18] Grillner S. Neuroscience. Human locomotor circuits conform. Science. 2011 Nov;334(6058):912-3.

[19] Harkema SJ. Plasticity of interneuronal networks of the functionally isolated human spinal cord. Brain Res Rev. 2008 Jan;57(1):255-64.

[20] Takakusaki K. Neurophysiology of gait: from the spinal cord to the frontal lobe. Mov Disord. 2013 Sep;28(11):1483-91.

[21] Ajemian S, Thon D, Clare P, Kaul L, Zernicke RF, Loitz-Ramage B. Cane-assisted gait biomechanics and electromyography after total hip arthroplasty. Arch Phys Med Rehabil. 2004 Dec;85(12):1966-71.

[22] Maguire C, Sieben JM, Frank M, Romkes J. Hip abductor control in walking following stroke -- the immediate effect of canes, taping and TheraTogs on gait. Clin Rehabil. 2010 Jan;24(1):37-45.

[23] Pearson KG, Misiaszek JE, Fouad K. Enhancement and resetting of locomotor activity by muscle afferents. Ann N Y Acad Sci. 1998 Nov;860:203-15.

[24] Delwaide PJ, Crenna P. Cutaneous nerve stimulation and motoneuronal excitability. II: Evidence for nonsegmental influences. J Neurol Neurosurg Psychiatry. 1984 Feb;47(2):190-6. 
[25] Dietz V. Spinal cord pattern generators for locomotion. Clin Neurophysiol. 2003 Aug;114(8):1379-89.

[26] Dietz V. Spinal cord lesion: effects of and perspectives for treatment. Neural Plast. 2001;8(1-2):83-90.

[27] Dietz V. Do human bipeds use quadrupedal coordination? Trends Neurosci. 2002 Sep;25(9):462-7.

[28] Lamoth CJ, van Deudekom FJ, van Campen JP, Appels BA, de Vries OJ, Pijnappels M. Gait stability and variability measures show effects of impaired cognition and dual tasking in frail people. J Neuroeng Rehabil. 2011;8:2.

[29] Sist B, Fouad K, Winship IR. Plasticity beyond peri-infarct cortex: spinal up regulation of structural plasticity, neurotrophins, and inflammatory cytokines during recovery from cortical stroke. Exp Neurol. 2014 Feb;252:47-56.

[30] Knikou M. Neural control of locomotion and training-induced plasticity after spinal and cerebral lesions. Clin Neurophysiol. 2010 Oct;121(10):1655-68.

[31] Enzinger C, Johansen-Berg H, Dawes H, Bogdanovic M, Collett J, Guy C, et al. Functional MRI correlates of lower limb function in stroke victims with gait impairment. Stroke. 2008 May;39(5):1507-13.

[32] Winstein C, Lewthwaite R, Blanton SR, Wolf LB, Wishart L. Infusing motor learning research into neurorehabilitation practice: a historical perspective with case exemplar from the accelerated skill acquisition program. J Neurol Phys Ther. 2014 Jul;38(3):190-200.

[33] Nudo RJ. Adaptive plasticity in motor cortex: implications for rehabilitation after brain injury. J Rehabil Med. 2003 May(41 Suppl):7-10.

[34] Kleim JA, Jones TA. Principles of experience-dependent neural plasticity: implications for rehabilitation after brain damage. J Speech Lang Hear Res. 2008 Feb;51(1):S225-39.

[35] Michael KM, Allen JK, Macko RF. Reduced ambulatory activity after stroke: the role of balance, gait, and cardiovascular fitness. Arch Phys Med Rehabil. 2005 Aug;86(8):1552-6.

[36] Patterson SL, Forrester LW, Rodgers MM, Ryan AS, Ivey FM, Sorkin JD, et al. Determinants of walking function after stroke: differences by deficit severity. Arch Phys Med Rehabil. 2007 Jan;88(1):115-9.

[37] Lee KB, Lim SH, Ko EH, Kim YS, Lee KS, Hwang BY. Factors related to community ambulation in patients with chronic stroke. Top Stroke Rehabil. 2015 Feb;22(1):63-71.

[38] Maki BE, Mcllroy WE. The role of limb movements in maintaining upright stance: the "change-insupport" strategy. Phys Ther. 1997 May;77(5):488-507.

[39] Maguire C, Sieben JM, Scheidhauer H, Romkes J, Suica Z, de Bie RA. The effect of crutches, an orthosis TheraTogs, and no walking aids on the recovery of gait in a patient with delayed healing post hip fracture: A case report. Physiother Theory Pract. 2016 Jan;32(1):69-81.

[40] Hart T, Bagiella E. Design and implementation of clinical trials in rehabilitation research. Arch Phys Med Rehabil. 2012 Aug;93(8 Suppl):S117-26.

[41] Folstein MF, Folstein SE, McHugh PR. "Mini-mental state". A practical method for grading the cognitive state of patients for the clinician. J Psychiatr Res. 1975 Nov;12(3):189-98.

[42] Tilson JK, Wu SS, Cen SY, Feng Q, Rose DR, Behrman AL, et al. Characterizing and identifying risk for falls in the LEAPS study: a randomized clinical trial of interventions to improve walking poststroke. Stroke. United States2012:446-52.

[43] Thieme H, Ritschel C, Zange C. Reliability and validity of the functional gait assessment (German version) in subacute stroke patients. Arch Phys Med Rehabil. United States2009:1565-70.

[44] Wrisley DM, Marchetti GF, Kuharsky DK, Whitney SL. Reliability, internal consistency, and validity of data obtained with the functional gait assessment. Phys Ther. 2004 Oct;84(10):906-18.

[45] Wrisley DM, Kumar NA. Functional gait assessment: concurrent, discriminative, and predictive validity in community-dwelling older adults. Phys Ther. 2010 May;90(5):761-73.

[46] Allum JH, Zamani F, Adkin AL, Ernst A. Differences between trunk sway characteristics on a foam support surface and on the Equitest ankle-sway-referenced support surface. Gait Posture. 2002 Dec;16(3):264-70.

[47] Allum JH, Carpenter MG. A speedy solution for balance and gait analysis: angular velocity measured at the centre of body mass. Curr Opin Neurol. 2005 Feb;18(1):15-21.

[48] Walker ML, Austin AG, Banke GM, Foxx SR, Gaetano L, Gardner LA, et al. Reference group data for the functional gait assessment. Phys Ther. 2007 Nov;87(11):1468-77. 


\section{Chapter 8}

[49] Lin JH, Hsu MJ, Hsu HW, Wu HC, Hsieh CL. Psychometric comparisons of 3 functional ambulation measures for patients with stroke. Stroke. 2010 Sep;41(9):2021-5.

[50] Leddy AL, Crowner BE, Earhart GM. Functional gait assessment and balance evaluation system test: reliability, validity, sensitivity, and specificity for identifying individuals with Parkinson disease who fall. Phys Ther. 2011 Jan;91(1):102-13.

[51] Deng H, Kimberley TJ, Durfee WK, Dressler BL, Steil C, Carey JR. Combined statistical analysis method assessing fast versus slow movement training in a patient with cerebellar stroke: a single-case study. Phys Ther. 2013 May;93(5):649-60.

[52] Nourbakhsh MR, Ottenbacher KJ. The statistical analysis of single-subject data: a comparative examination. Phys Ther. 1994 Aug;74(8):768-76.

[53] Backman CL, Harris SR. Case studies, single-subject research, and N of 1 randomized trials: comparisons and contrasts. Am J Phys Med Rehabil. 1999 Mar-Apr;78(2):170-6.

[54] Marklund I, Klässbo M. Effects of lower limb intensive mass practice in poststroke patients: single-subject experimental design with long-term follow-up. Clin Rehabil. 2006 Jul;20(7):568-76.

[55] Byiers BJ, Reichle J, Symons FJ. Single-subject experimental design for evidence-based practice. Am J Speech Lang Pathol. 2012 Nov;21(4):397-414.

[56] Ustun TB, Chatterji S, Bickenbach J, Kostanjsek N, Schneider M. The International Classification of Functioning, Disability and Health: a new tool for understanding disability and health. Disabil Rehabil. 2003 Jun 3-17;25(11-12):565-71.

[57] Horlings CG, van Engelen BG, Allum JH, Bloem BR. A weak balance: the contribution of muscle weakness to postural instability and falls. Nat Clin Pract Neurol. 2008 Sep;4(9):504-15.

[58] Recktenwald MR, Hodgson JA, Roy RR, Riazanski S, McCall GE, Kozlovskaya I, et al. Effects of spaceflight on rhesus quadrupedal locomotion after return to 1G. J Neurophysiol. 1999 May;81(5):2451-63.

[59] Goutier KM, Jansen SL, Horlings CG, Küng UM, Allum JH. The influence of walking speed and gender on trunk sway for the healthy young and older adults. Age Ageing. 2010 Sep;39(5):647-50. 
Chapter

General Discussion 


\section{THE NEED FOR EFFECTIVE AND INEXPENSIVE INTERVENTIONS}

Improving the effectiveness of gait and balance rehabilitation following stroke, with inexpensive interventions, needs to be a global priority. The reduction of disability, the prevention of secondary complications, which lead to loss of independence and depression, and consequently an easing of the global burden of disease could all be improved if low-priced, effective treatments were widely available. This is particularly the case as most of the burden of stroke falls on low and middle income countries and the incidence of stroke is increasing in these economies.[1] Despite this, much recent research concentrates on resource intensive interventions such as robotics [2-4], virtual reality and computer games $[5,6]$ and various forms of non-invasive brain stimulation such as transcranial direct current, transcranial magnetic and paired associative stimulations. [7, 8] Although the development of such interventions is crucial to supplement or increase the efficacy of current treatments, the availability of such interventions is likely to remain limited to well resourced, first world economies. Even in these economies, financial pressures on health care providers mean that treatments must be cost effective and expensive interventions may be rationed. [9] Therefore, as well as developing new treatments, the efficacy and optimal utilization of current, widespread aspects of care should be evaluated. Improving the evidence based choice and use of walking aids may provide an excellent opportunity to cost-effectively improve patient outcomes and thus relieve pressure on resources.

\section{TRANSLATING NEUROSCIENTIFIC EVIDENCE INTO CLINICAL HYPOTHESES}

Walking aids are widely used in gait rehabilitation following stroke. However, prior to the studies included in this thesis, no prospective, experimental studies had been conducted to evaluate the long-term effects of walking aids on balance and gait recovery. Experimental studies which had been conducted were limited to cross-sectional designs, predominantly evaluating the kinetic and kinematic effects of walking aids. The theoretical backgrounds presented in these studies were consequently biomechanical in nature.

We felt that as stroke patients have pathological changes in the central nervous system (CNS), affecting control of walking and balance, the impact of walking aids on these neuropathological processes should be considered. Chapters $2 \& 3$ as well as the introductions of chapters $7 \& 8$ therefore present a theoretical framework of the neurophysiological and neuromuscular control of gait and balance in both healthy and post-stroke individuals. Processes of recovery and factors influencing recovery are also discussed. This systematic examination of the neural science enabled the formulation of hypotheses regarding long-term effects of walking aids on gait function. The study protocol in chapter 7 and the study in chapter 8 therefore set out to analyse the impact of walking 
aids in stroke from this novel perspective. The studies in chapters 4 \& 5 examined the impact of various walking aids on healthy subjects or subjects undergoing rehabilitation without neurological impairments. The results of these studies enabled a deeper understanding of the immediate and long-term effects of walking aids on factors hypothesized to be important for post-stroke recovery, e.g. levels of muscle activity and balance control, in non-neurologically impaired subjects. Understanding how "normal" subjects responded enabled a more precise formulation of hypotheses for stroke patients which are outlined in the following sections.

\section{ASPECTS OF NEURAL CONTROL IDENTIFIED AS IMPORTANT IN POST- STROKE GAIT RECOVERY}

\section{Motor learning and re-learning}

Motor learning processes are operative in the acquisition of new skills post-stroke, both during spontaneous recovery and in rehabilitative interventions.[10, 11] Although this has been widely demonstrated and has been the basis for the development of several effective treatments such as robotic therapy, [12] virtual reality [13] and constrained induced movement therapy, [14] the principles had not been applied to our knowledge, in studies previous to this thesis, to investigate the role of walking aids in gait rehabilitation.

We considered that many of the criteria known to be important to support motorlearning, such as high repetition, $[10,15,16]$ task specific but variable training [16] completing a meaningful task (in this case walking), [15] are fulfilled when walking with aids throughout the day. On this basis we hypothesized that the influence of walking aids on gait function may be considerable, therefore justifying the examination of these specific effects.

\section{Neuronal plasticity}

We considered aspects of cortical and spinal plasticity to postulate what influence walking aids may have on plasticity and how this may affect gait and balance recovery and function.

\section{Cortical plasticity}

Cortical plasticity following stroke has been widely studied in both animal and human models. Animal studies have shown that improvements in motor skills lead to dendritic growth, synaptogenesis, enhanced synaptic responses and expansion of motor task representation in the cerebral cortex.[17, 18] Studies in humans have also shown that 
increased neuronal activity in the lesioned cortical hemisphere (indicating motor-map activation in or near the pre-infarct task representation ) during movement of the hemiplegic side, correlates with better function. Equally, increased activity in the nonlesioned hemisphere correlates with poorer motor recovery and functional outcomes. $[19,20]$ This suggests that rehabilitation should attempt to induce plasticity in the lesioned hemisphere, possibly the original neuronal networks or closely associated networks, to optimise function. A meta-analysis [21] reviewed interventions in the upperlimb which lead to increased activity in the ipsilesional cortical hemisphere during arm movement. It was concluded that "neural changes in the sensorimotor cortex of the lesioned hemisphere were achieved with rehabilitation interventions that emphasized use of the paretic upper limb and resulted in improved functional motor gains." [21] On the basis of this evidence we hypothesised that, due to the same mechanism, walking aids which increase activity on the hemiplegic side would be more likely to induce ipsilesional cortical plasticity and induce functional gains than walking aids which reduce hemiplegic side activity.

\section{Spinal plasticity}

Spinal cord plasticity and factors affecting this have been frequently investigated in spinal cord injury patients, but much less in stroke patients. In chapter 2, spinal networks controlling gait and factors which influence this control were reviewed before and after injury to the CNS. Neuronal plasticity at spinal cord level and resultant effects on cortical plasticity and function were also considered. On the basis of the review findings we postulated that walking aids which (1) allowed normal joint and muscle loading, (2) allowed normal cutaneous feedback and (3) did not use hands would have the most positive effects on plasticity in the spinal neuronal networks controlling gait and consequently also on cortical plasticity and function.

\section{Theory to practice}

Based on the theoretical analysis of the above mentioned aspects of neural science we concluded that walking aids:

- Are likely to have a significant and specific effect on gait and balance recovery following stroke.

- To be optimally effective should not reduce muscle activity on the hemiplegic side, but rather should increase this muscle activity.

- Should not require the use of hands.

- Should allow normal joint and muscle loading.

- Should allow normal cutaneous feedback. 
We therefore hypothesized that an orthotic garment and strapping system such as "TheraTogs" would be more effective in gait rehabilitation following stroke than canes to restore gait function and balance.

\section{Testing the theory in acute and chronic patients}

Recovery after stroke can occur in both acute and chronic patients. [10, 22, 23]

\section{Acute patients}

Early after stroke spontaneous recovery begins. The majority of spontaneous recovery has been observed to occur between 8 [24] and 10 weeks [25] post-stroke and has been estimated to be responsible for between $16 \%$ and $42 \%$ of variance in functional outcomes at 10 weeks. [26] A typical non-linear recovery pattern over time is observed, showing steep increases in the first 10 weeks, slowing to 20 weeks after which spontaneous recovery plateaus. [27] This pattern suggests an underlying mechanism of recovery that impacts final outcome. Physiological processes involved in this spontaneous recovery appear to include 1.restoration of function in penumbral (non-infarcted) areas, 2. resolution of diaschisis (temporary loss of function in other non-damaged CNS regions due to loss of connectivity), 3.tissue repair via neural plasticity.[28]

Nudo states that a time-dependent cascade of reactions involving morphological changes is set into motion in the peri-infarct and remote regions by focal ischemic damage to the cerebral cortex. [29] Early after injury, growth promoting molecules are demonstrated. In the second to third week post-stroke, dendritic sprouting is observed in the contralesional hemisphere and widespread synaptogenesis is seen by the third week. This time dependent sequence of events creates a plasticity-promoting environment, which is responsible for spontaneous recovery. However, plasticity is also influenced by post-infarct behavior and rehabilitation. [30] Intensive rehabilitation increased cortical hand representation in infarct-induced primates compared to a norehabilitation control group, in which hand representation decreased. [31] It would seem logical therefore, that the efficacy of interventions would be increased if carried out during the plasticity promoting phase. It has indeed been shown in animal models, that training is most effective at restoring pre-infarct function if begun within one week of injury. [22] It has also been shown that delaying the initiation of treatment until 25 or 30 days post-infarct results in reduced dendritic branching and poorer functional recovery than training initiated 4 days post-injury. $[32,33]$ It has been found that a statistically significant negative association exists between effect sizes in individual trials and timing of the first therapeutic intervention. [34]

When determining the methods for the studies in this thesis, we therefore concluded that one study should evaluate the influence of different walking aids during gait rehabilitation in acute patients. Chapter 7 describes the protocol for this study. As pa- 
tients recover functional abilities at different times, inclusion criteria was not defined as time since stroke, rather as gait function described as Functional Ambulation level 3 (patients just beginning to walk with supervision or with minimal contact). This was the point at which therapists would first have to decide which walking aid to prescribe. As spontaneous recovery would still be occurring at this point, it was decided that a randomized control trial, in which the effect of two different walking aids (canes and TheraTogs) would be compared was the optimal design.

\section{Chronic patients}

Spontaneous recovery appears to plateau at approximately 20 weeks post-stroke [26] and it has been claimed, on the basis of observational studies, that no further functional recovery should be expected after 5 months. [35] However, although a plasticity enriched environment no longer exists after 4-5 months, usual mechanisms of motor learning are still able to occur in chronic stroke patients. As is the case with healthy individuals who reach "plateaus" when learning new motor skills or reaching levels of fitness, modifying aspects of training such as variety of exercises, context or intensity of practice can result in motor and fitness gains. [23] Although it is correct that chronic stroke patients often cease to improve in functional ability after 5 months, it is not correct to assume that this is indicates a reduced capacity to learn. The assumption that no further gains will be made has often lead to the cessation of therapy in this patient group, creating a self-fulfilling prophesy.[23] Recent studies have demonstrated that improvements can be achieved in chronic stroke patients. [36-38] We therefore concluded that one study in this thesis should evaluate the impact of different walking aids on chronic stroke patients.

We earlier hypothesized that walking aids such as canes which required hand use (decoupling from four-limb CPG control and requiring increased cognitive resources), reduced hemiplegic muscle activity when held in the non-hemiplegic hand (potentially reducing ipsilesional plasticity and functional outcomes), unloaded hemiplegic joints and muscles (adversely effecting peripheral afferents and CPG output), and increased base of support (reducing need to use intrinsic balance reactions) would not optimally improve gait function. An orthosis TheraTogs which does not require hand use, increases hemiplegic muscle activity during gait, does not unload muscles or joints and does not increase base of support, may have a more positive effect. As variety and intensity of exercise as well as changes in context may promote motor learning in chronic stroke patients, we hypothesized that changing canes to TheraTogs would improve gait function even in chronic stroke patients.

We determined that a multiple single case study design would be appropriate to investigate this clinical question. As all patients had passed the stage of spontaneous recovery, a stable baseline period of gait function when walking with canes could be 
assumed. Inclusion criteria therefore included chronic, cane using (always outside, usually inside) stroke patients. This study is described in chapter 8.

\section{Study designs for rehabilitation. Randomized, control trials and single case designs.}

Although randomized control trials are considered the gold standard for studies aiming to test the effectiveness of interventions, $[39,40]$ the practical realities of completing such studies in a rehabilitation setting can mean that intervention contamination is difficult to adequately exclude [41] and that statistical power can be compromised due to recruitment difficulties [42]. This is reflected in the results of Cochrane reviews, systematic reviews and meta-analyses regarding rehabilitation interventions in which clear conclusions are often lacking and appeals for further studies are relatively common.[4345] Hart et al.[41] discuss some of the added complexities of rehabilitation research compared to pharmaceutical or surgical studies, such as the need for active engagement and effort on the part of the patient compared to passive interventions in the former, the interpersonal relationship between patient and therapist as well as the collective behaviour of the rehabilitation team.

\section{Randomized control trial}

In the RCT carried out according to the protocol in this thesis, many of the problems discussed in the literature were experienced. The RCT was initially planned to include three neurological rehabilitation units as part of a multi-center trial. This was later increased to four. Although dedicated staff were appointed in each center to screen all stroke admissions for suitability for inclusion, recruitment numbers were poor from the outset. Several factors influenced this. The most significant being the narrow patient profile defined by the inclusion and exclusion criteria. In order to ensure good internal validity, factors such as many co-morbidities, previous strokes or common symptoms such as neglect were excluded. The subject defined by these criteria did not represent the majority of stroke patients appearing in the clinics. Additionally, therapist attitudes were frequently a negative factor. Therapists working in the rehabilitation teams often had opinions, based on clinical experience, regarding the efficacy of the two walking aids being tested. If they felt that one of the aids was not appropriate for their patient (randomization meant that the chance of receiving that intervention was 50\%), they prevented inclusion into the study. This was done for example by suggesting to the patient or relatives that inclusion would not be in their best interest. This was not done maliciously but nevertheless increased recruitment problems.

Recruitment is a general problem in rehabilitation studies. Our experience illustrates that the requirement to improve internal validity through strictly defined inclusion and exclusion criteria can lead to lack of statistical power or (if power is achieved), may lead 
to study results in a population not representative of clinical reality. Pragmatic RCTs, designs in which inclusion and exclusion criteria are more loosely defined in order to represent the real world patient population, could offer solutions to these problems. [46] It may be that the inclusion of pragmatic RCT designs into the rehabilitation research portfolio would help to avoid recruitment and generalizability difficulties in future trials.

The importance of therapist attitudes was also illustrated. We realized in advance that the support of team members for the study could be decisive and therefore interventions to achieve this were included in the methods. All teams were given intensive training providing a theoretical background to the study and on-going support was offered for therapists whose patients had been included. Thorough discussions took place regarding the important difference between decisions based on clinical experience or evidence, and the need to improve the external evidence base of clinical practice. Nevertheless, therapists often felt in an ethical dilemma if in their clinical opinion an intervention was not optimal for patient recovery.

Contamination was also a challenge in this design. Subjects randomized to the TheraTogs group were fitted daily with the orthosis and were not supposed to walk with canes. Nursing and therapy staff were given practical training regarding application, booklets with photographs were provided and illustrated instructions were wall mounted by subjects beds. Despite this, application of the orthosis varied greatly. Factors such as staff changes and time restraints in busy departments contributed to this problem. Dedicated personnel to apply the orthosis in each of the four centers might have helped to improve standardization. However, this would have required financial resources which were not available. Subjects were occasionally given canes by staff members or relatives not fully aware of the protocol such as temporary agency staff. These issues indicate that to reduce contamination and ensure adherence to an intervention in multicenter trials, significant financial resources to create a stable study infra-structure are required. Such resources are rarely available in physiotherapy or rehabilitation studies.

\section{Alternative designs for physiotherapy and rehabilitation research}

In order to supplement the findings of RCTs and to provide additional and robust evidence for the efficacy of rehabilitation interventions, alternative study designs are necessary. Pragmatic RCTs have been mentioned. A further possibility are small sample designs. The potential for small-sample or small-N designs to fill this role has been widely discussed in research literature. $[47,48]$ This design is often used in behavioural sciences and psychotherapy but remains relatively rare in primary and secondary care research. Small-sample studies involve frequent and systematic measurement of patients before, during and following an intervention. Baseline values of the dependent variable are established before intervention begins, changes occurring during and postintervention can be described and analyzed by comparison to baseline values. In RCTs 
the effectiveness of an intervention is tested by means of a comparison of dependent variables (means or differences) between intervention and control group. In a single case design the patient is used as his or her own control - the values of the dependent variable during and after intervention are compared to the baseline values before treatment, each case being an experiment in itself. Based on this intra-subject variation, effectiveness of the intervention can be reliably tested. [42, 49]. Potential ethical dilemmas for therapy staff are reduced as all patients receive the intervention and smaller subject numbers who are seen frequently by research staff (for intervention or measurement), mean that possible sources of contamination are easier to exclude. Generalizability can be improved by replication with multiple single cases. The statistical power of single case studies lies in the number of measurements, not in the number of subjects as in classical RCT designs. As patients are their own control, strict inclusion and exclusion criteria do not need to be defined as comorbidities generally remain constant throughout phases. If not, this can be noted and included in the data evaluation and interpretation.

Several designs have been applied in research including $A B$ design - $A$ being control condition, $B$ being intervention, $A B A$ design, $A B A B$ design, $A B C$ design in which $C$ is a further intervention or follow-up etc. $A B C$ designs are recommended for interventions which are expected to show stable changes.

\section{Multiple single case study}

In chapter 8 we present a multiple single case study in which the effect of different walking aids on gait function and balance in chronic stroke patients is presented. The results show an improvement in gait function during the intervention phase in all patients. The improvement is sustained in the follow-up phase in three from four subjects.

\section{Comparison of these designs}

As a neuroplasticity supporting environment exists with acute post-stroke patients, it is easier to influence change in this patient group than in chronic patients. It could therefore be expected that as effect sizes are likely to be bigger in acute patients, it would be easier to show a between-group difference in an RCT with acute patients than a between-phase difference with chronic patients in a multiple single case study. However in the studies we conducted, this was not the case. In the RCT, due to the problems discussed above, recruitment numbers were very low. In total, 11 patients over a period of 18 months were recruited from the four study clinics (Felix Platter Spital, Kantonspital Luzern, Reha BadZurzach, Kantonspital Bruderholz). A lack of statistical power as well as high levels of contamination therefore made it impossible to establish between group differences. A difference was established however between phases, with chronic stroke patients in the replicated single case design. These results suggest that 
the replicated single case design was able to demonstrate a (probably smaller) effect size and between phases difference, in a group of patients with whom change was relatively difficult to achieve. The RCT was unable to demonstrate a (likely larger) effect size and between group difference, in a group of patients in whom change was easier to affect. In the investigation of the clinical question asked in this thesis "are TheraTogs more effective than canes to improve gait function and balance in stroke patients?" the multiple single case design was superior to the RCT to demonstrate differences.

As the need to demonstrate the effectiveness in rehabilitation interventions continues to grow, this result is important for the planning of future research designs. It may be that traditional Levels of Evidence [40] should not only state the adequacy of study designs for particular clinical questions, e.g RCT for intervention studies, analytic crosssectional studies for diagnostic studies etc., rather also for the clinical area being investigated. It may be that RCTs are the optimal primary research design for medication and surgical interventions but not for multi-faceted rehabilitative interventions. Replicated single case designs and pragmatic RCTs may be more appropriate and at least equally powerful for these questions.

\section{Results, future research and clinical practice guidelines}

The results of the studies presented in this thesis demonstrate that some walking aids, namely canes, rollators and crutches have the immediate effect of reducing muscle activity when walking compared to walking with no aids. Others, namely TheraTogs and tape increase muscle activity when walking compared to walking with no aids. This may have consequences for the long-term development of muscle strength and for neural plasticity.

In rollators we demonstrated that trunk sway, a measure indicating balance when walking, was not reduced, although pelvic muscle activity was lower, which normally leads to reduced pelvic stability and increased trunk sway. This suggests that stability was gained elsewhere, likely from increased arm activity through pushing on the rollator. This suggests that rollator walking facilitates abnormal balance reactions. A similar response is likely in all walking aids which use arms.

As walking aids which use arms increase the base of support, stability is increased via this external factor, giving an immediate impression of increased stability for patients, but reducing the need to use inherent balance reactions. In the long-term, this could reduce balance.

On the basis of the theoretical reviews in this thesis we also postulate that walking aids which use hands require more cognitive resources as decoupling from four limb CPG control is necessary. This is problematic in patients with reduced balance who often use more cognitive resources to stay stable and frequently have difficulty with dual tasking, for example. 
We also postulated that walking aids which enable normal afferent inputs are more likely to facilitate normal motor output from spinal CPG circuits. Walking aids which do not use hands such as TheraTogs, allow normal joint and muscle loading and do not adversely affect cutaneous and kinematic feedback. Walking aids which use hands alter many of these parameters, therefore causing abnormal afferent input when walking and potentially disrupting the output of spinal circuitry.

We report the results from two studies, one a single case report with an orthopedic patient and one a multiple single case study with chronic stroke patients, which investigated experimentally, and to our knowledge for the first time, the long term effect of different walking aids on gait and balance. The results appeared to confirm our hypotheses. Walking with TheraTogs when walking without cane or with reduced cane use improved parameters of gait function and balance in all patients, although effect sizes varied.

\section{CONCLUSIONS}

Walking aids appear to have a specific and significant effect on the control of gait and balance. Walking aids which don't use hands appear to be more effective than canes or rollators in gait rehabilitation for stroke patients to improve walking function and balance. Additional experimental research is needed in more patients to establish generalisable results across different types of patient profiles and to further optimize intervention details such as duration or frequency/intensity of walking aid use.

Some aspects of the effects of walking aids still need to be clarified. It remains unclear what the long term effect is, of walking with aids which reduce lower limb muscle activity. It may be that muscle strength is reduced. On the other hand, for patients who would not walk at all without canes or rollators, muscle strength would improve compared to being sedentary.

For patients with fear of falling or with perceptual difficulties, it may not be possible to walk without the feeling of stability provided by canes or rollators. Walking aids which do not provide the external stability delivered by hand held devices, may not be appropriate for such patients.

However, as we have demonstrated, the decisive impact of walking aids on the longterm outcomes of gait function and balance, the need for further research to answer these questions and thus enable the appropriate, evidence based prescription of walking aids has been shown.

As our results confirm findings of observational studies that cane using stroke patients demonstrate poorer balance and gait function than non-cane using stroke patients, clinical practice guidelines need to be changed to reflect this. The recommendation that "in the opinion of the guideline development team, the use of walking-aids is 
beneficial to patients with a stroke in terms of safety, independence, and efficiency of walking, as well as confidence" [10] should be reviewed.

The results presented in this thesis offer clues towards a cost-effective method to help improve gait and balance in stroke patients. The concept that hand-free devices are more effective that traditional canes or rollators in certain patient groups, may enable the development of similar appliances to TheraTogs. Walking aids which help improve gait function and balance may reduce disability and help improve quality of life in acute and chronic stroke patients. 


\section{REFERENCES}

1. Feigin VL, Forouzanfar MH, Krishnamurthi R, Mensah GA, Connor M, Bennett DA, Moran AE, Sacco RL, Anderson L, Truelsen T et al: Global and regional burden of stroke during 1990-2010: findings from the Global Burden of Disease Study 2010. Lancet 2014, 383(9913):245-254.

2. Swinnen E, Beckwee D, Meeusen R, Baeyens JP, Kerckhofs E: Does robot-assisted gait rehabilitation improve balance in stroke patients? A systematic review. Top Stroke Rehabil 2014, 21(2):87-100.

3. Forrester LW, Roy A, Goodman RN, Rietschel J, Barton JE, Krebs HI, Macko RF: Clinical application of a modular ankle robot for stroke rehabilitation. NeuroRehabilitation 2013, 33(1):85-97.

4. Mehrholz J, Elsner B, Werner C, Kugler J, Pohl M: Electromechanical-assisted training for walking after stroke. Cochrane Database Syst Rev 2013, 7:Cd006185.

5. Luque-Moreno C, Ferragut-Garcias A, Rodriguez-Blanco C, Heredia-Rizo AM, Oliva-Pascual-Vaca J, Kiper P, Oliva-Pascual-Vaca A: A Decade of Progress Using Virtual Reality for Poststroke Lower Extremity Rehabilitation: Systematic Review of the Intervention Methods. Biomed Res Int 2015, 2015:342529.

6. Laver KE, George S, Thomas S, Deutsch JE, Crotty M: Virtual reality for stroke rehabilitation. Cochrane Database Syst Rev 2015, 2:Cd008349.

7. Wessel MJ, Zimerman M, Hummel FC: Non-invasive brain stimulation: an interventional tool for enhancing behavioral training after stroke. Front Hum Neurosci 2015, 9:265.

8. Tan AQ, Shemmell J, Dhaher YY: Downregulating Aberrant Motor Evoked Potential Synergies of the Lower Extremity Post Stroke during TMS of the Contralesional Hemisphere. Brain Stimul 2016.

9. Rooshenas L, Owen-Smith A, Hollingworth W, Badrinath P, Beynon C, Donovan JL: "I won't call it rationing...": an ethnographic study of healthcare disinvestment in theory and practice. Soc Sci Med 2015, 128:273-281.

10. Krakauer JW: Motor learning: its relevance to stroke recovery and neurorehabilitation. Curr Opin Neurol 2006, 19(1):84-90.

11. Winstein CJ, Merians AS, Sullivan KJ: Motor learning after unilateral brain damage. Neuropsychologia 1999, 37(8):975-987.

12. Fasoli SE, Krebs HI, Stein J, Frontera WR, Hogan N: Effects of robotic therapy on motor impairment and recovery in chronic stroke. In: Arch Phys Med Rehabil. Volume 84, edn. United States; 2003: 477-482.

13. Holden MK: Virtual environments for motor rehabilitation: review. Cyberpsychol Behav 2005, 8(3):187211; discussion 212-189.

14. Mark VW, Taub E: Constraint-induced movement therapy for chronic stroke hemiparesis and other disabilities. Restor Neurol Neurosci 2004, 22(3-5):317-336.

15. Plautz EJ, Milliken GW, Nudo RJ: Effects of repetitive motor training on movement representations in adult squirrel monkeys: role of use versus learning. Neurobiol Learn Mem 2000, 74(1):27-55.

16. Hubbard IJ, Parsons MW, Neilson C, Carey LM: Task-specific training: evidence for and translation to clinical practice. Occup Ther Int 2009, 16(3-4):175-189.

17. Nudo RJ: Adaptive plasticity in motor cortex: implications for rehabilitation after brain injury. J Rehabil Med 2003(41 Suppl):7-10.

18. Kleim JA, Jones TA: Principles of experience-dependent neural plasticity: implications for rehabilitation after brain damage. J Speech Lang Hear Res 2008, 51(1):S225-239.

19. Enzinger C, Johansen-Berg H, Dawes H, Bogdanovic M, Collett J, Guy C, Ropele S, Kischka U, Wade D, Fazekas $\mathrm{F}$ et al: Functional MRI correlates of lower limb function in stroke victims with gait impairment. Stroke 2008, 39(5):1507-1513.

20. Ward NS, Cohen LG: Mechanisms underlying recovery of motor function after stroke. Arch Neurol 2004, 61(12):1844-1848.

21. Richards LG, Stewart KC, Woodbury ML, Senesac C, Cauraugh JH: Movement-dependent stroke recovery: a systematic review and meta-analysis of TMS and fMRI evidence. Neuropsychologia 2008, 46(1):3-11.

22. Nudo RJ: Mechanisms for recovery of motor function following cortical damage. Curr Opin Neurobiol 2006, 16(6):638-644. 
23. Page SJ, Gater DR, Bach YRP: Reconsidering the motor recovery plateau in stroke rehabilitation. Arch Phys Med Rehabil 2004, 85(8):1377-1381.

24. van Kordelaar J, van Wegen E, Kwakkel G: Impact of time on quality of motor control of the paretic upper limb after stroke. Arch Phys Med Rehabil 2014, 95(2):338-344.

25. Kwakkel G, Kollen BJ: Predicting activities after stroke: what is clinically relevant? Int J Stroke 2013, $8(1): 25-32$.

26. Kwakkel G, Kollen B, Twisk J: Impact of time on improvement of outcome after stroke. Stroke 2006, 37(9):2348-2353.

27. Kwakkel G, Kollen B, Lindeman E: Understanding the pattern of functional recovery after stroke: facts and theories. Restor Neurol Neurosci 2004, 22(3-5):281-299.

28. Kwakkel G, Kollen B, Lindeman E: Understanding the pattern of functional recovery after stroke: facts and theories. Restor Neurol Neurosci 2004, 22(3-5):281-299.

29. Nudo RJ: Plasticity. NeuroRx 2006, 3(4):420-427.

30. Nudo RJ: Postinfarct cortical plasticity and behavioral recovery. Stroke 2007, 38(2 Suppl):840-845.

31. Nudo RJ, Milliken GW, Jenkins WM, Merzenich MM: Use-dependent alterations of movement representations in primary motor cortex of adult squirrel monkeys. J Neurosci 1996, 16(2):785-807.

32. Biernaskie J, Chernenko G, Corbett D: Efficacy of rehabilitative experience declines with time after focal ischemic brain injury. J Neurosci 2004, 24(5):1245-1254.

33. Hsu JE, Jones TA: Time-sensitive enhancement of motor learning with the less-affected forelimb after unilateral sensorimotor cortex lesions in rats. Eur J Neurosci 2005, 22(8):2069-2080.

34. Ottenbacher KJ, Jannell S: The results of clinical trials in stroke rehabilitation research. Arch Neurol 1993, $50(1): 37-44$.

35. Jorgensen HS, Nakayama H, Raaschou HO, Vive-Larsen J, Stoier M, Olsen TS: Outcome and time course of recovery in stroke. Part II: Time course of recovery. The Copenhagen Stroke Study. Arch Phys Med Rehabil 1995, 76(5):406-412.

36. Macko RF, Ivey FM, Forrester LW, Hanley D, Sorkin JD, Katzel LI, Silver KH, Goldberg AP: Treadmill exercise rehabilitation improves ambulatory function and cardiovascular fitness in patients with chronic stroke: a randomized, controlled trial. Stroke 2005, 36(10):2206-2211.

37. Whitall J, McCombe Waller S, Silver KH, Macko RF: Repetitive bilateral arm training with rhythmic auditory cueing improves motor function in chronic hemiparetic stroke. Stroke 2000, 31(10):2390-2395.

38. Malagoni AM, Cavazza S, Ferraresi G, Grassi G, Felisatti M, Lamberti N, Basaglia N, Manfredini F: Effects of a "test in-train out" walking program versus supervised standard rehabilitation in chronic stroke patients: a feasibility and pilot randomized study. Eur J Phys Rehabil Med 2016.

39. Trisha G: How to Read a Paper: The Basics of Evidence based Medicine, 3rd Edition edn: John Wiley and Sons, Blackwell Publishing, BMJ Books; 2006.

40. The Oxford 2011 Levels of Evidence

41. Hart T, Bagiella E: Design and implementation of clinical trials in rehabilitation research. Arch Phys Med Rehabil 2012, 93(8 Suppl):S117-126.

42. Graham JE, Karmarkar AM, Ottenbacher KJ: Small sample research designs for evidence-based rehabilitation: issues and methods. Arch Phys Med Rehabil 2012, 93(8 Suppl):S111-116.

43. Nair RD, Lincoln NB: Cognitive rehabilitation for memory deficits following stroke. Cochrane Database Syst Rev 2007(3):CD002293

44. Mehrholz J, Kugler J, Pohl M: Water-based exercises for improving activities of daily living after stroke. Cochrane Database Syst Rev 2011(1):CD008186.

45. Brasure M, Lamberty GJ, Sayer NA, Nelson NW, Macdonald R, Ouellette J, Wilt TJ: Participation after Multidisciplinary Rehabilitation for Moderate to Severe Traumatic Brain Injury in Adults: A Systematic Review. Arch Phys Med Rehabil 2013.

46. Woodcock A, Bakerly ND, New JP, Gibson JM, Wu W, Vestbo J, Leather D: The Salford Lung Study protocol: a pragmatic, randomised phase III real-world effectiveness trial in asthma. BMC Pulm Med 2015, 15(1):160. 
47. Barnett SD, Heinemann AW, Libin A, Houts AC, Gassaway J, Sen-Gupta S, Resch A, Brossart DF: Small N designs for rehabilitation research. J Rehabil Res Dev 2012, 49(1):175-186.

48. Horn SD, DeJong G, Deutscher D: Practice-based evidence research in rehabilitation: an alternative to randomized controlled trials and traditional observational studies. Arch Phys Med Rehabil 2012, 93(8 Suppl):S127-137.

49. Bloom M, J. F, G OJ: Evaluating practice: guidelines for the accountable professional (6th ed.)Allyn and Bacon, Boston edn; 2009.

50. Guyatt GH, Keller JL, Jaeschke R, Rosenbloom D, Adachi JD, Newhouse MT: The n-of-1 randomized controlled trial: clinical usefulness. Our three-year experience. Ann Intern Med 1990, 112(4):293-299. 

Summary 
Chapter 1. In the general introduction, the prevalence of stroke, resulting disability and global burden of disease are discussed in order to highlight the need for effective and affordable treatment interventions. The requirement of a clear understanding of neurophysiological processes as a basis for the development of effective treatments is debated. These thoughts are considered both in the context of the International Classification of Function (ICF) and in relation to the historical development of many physiotherapeutic, rehabilitative interventions. Current research into walking aids is summarized, indicating that the majority of experimental studies are cross-sectional, testing the immediate, biomechanical effects of assistive devices. It is shown that prospective, experimental studies which consider the neurophysiological impact of walking-aids are lacking. Finally, the aims of the thesis are described.

Chapter 2. In this chapter we reviewed the structure and function of spinal interneuronal networks and Central Pattern Generators with respect to walking in healthy and post-stroke patients. The influence of afferent inputs (essential for proper gait) on these structures was discussed. Recent evidence demonstrating spinal post-stroke plasticity was presented and its relevance for gait recovery and the possible influence of walking aids on these mechanisms were considered. The aim of this review was to relate, as far as we are aware for the first time, knowledge from basic neuroscience and clinical research done with other patient groups (primarily spinal cord injury patients) to stroke rehabilitation. We discussed recent studies which indicate that changes in spinal cord circuitry post-stroke contribute to functional recovery. We presented evidence which suggests that peripheral inputs such as joint and muscle loading and muscle activity during gait can influence spinal plasticity and therefore impact motor recovery. We suggest that peripheral afferent information should be as similar as possible to input during healthy gait in order to positively impact spinal plasticity and functional recovery. We concluded that assistive walking devices should allow normal joint loading, not reduce muscle activity or length during walking and not require the use of hands. We show that canes and rollators negatively impact all of these afferent inputs whereas an orthosis TheraTogs, allows normal joint and muscle loading, increases muscle activity and does not require the use of hands. TheraTogs and similar orthoses may therefore be more effective in gait rehabilitation. This extrapolation of knowledge was an attempt to improve the understanding of neural, neuromuscular and pathophysiological processes which occur when walking with different devices following stroke. This may help to optimize prescription and use of walking aids in rehabilitation.

Chapter 3. In this chapter we reviewed aspects of cortical control of gait and movement, and focused on muscle synergies, as a way of translating cortical commands into specific muscle activity and as an efficient means of reducing neural and musculoskeletal redundancy. We presented evidence which suggests that four to five muscle synergies are responsible for the normal control of walking and running in all conditions and are stored in the spinal cord as Central Pattern Generators. We summarized evidence showing that post-stroke these synergies appear initially to remain intact at spinal-cord 
level, but that the ability to selectively recruit and combine them in flexible ways to serve the motor task, is lost. It appears that this selective recruitment is primarily under supra-spinal and cortical control but also influenced by peripheral inputs. As spinal plasticity occurs post-stroke and is activity dependent, the repeated recruitment of combined muscle synergies may lead to a permanent loss of selective and adaptable gait control. We suggest that normalizing peripheral input during gait may facilitate the selective recruitment of spinal Central Pattern Generators (gait muscle synergies), potentially compensating for abnormal cortical inputs and helping to maintain or restore selective recruitment. The need for walking aids to allow normal afferent information from the periphery, as discussed in chapter 2 was therefore re-emphasized. The disadvantages of canes and rollators and the advantages of TheraTogs were again debated. This chapter was a further attempt to improve and broaden the understanding of neurophysiological and pathophysiological processes which occur following stroke in the control of walking and how these processes are relevant for the optimal prescription and use of walking aids in rehabilitation.

Chapter 4. In this cross-sectional study in post-stroke individuals, we investigated the immediate effect of walking with canes, tape and an orthotic garment TheraTogs on hip abductor muscle activity on the hemiplegic side and on tempero-spatial gait parameters, compared to walking with no aids. We found that walking with canes in the hemiplegic hand significantly reduced muscle activity on the hemiplegic side compared to walking with no aids. Walking with tape and TheraTogs significantly increased hemiplegic muscle activity compared to walking with no aids. Increases in muscle activity were greatest with TheraTogs. No significant changes in tempero-spatial gait parameters between conditions were found. As other studies have indicated that increasing muscle activity on the hemiplegic side increases ipsilesional cortical plasticity, which correlates to better function, these findings were potentially important when considering how walking aids should be prescribed post-stroke to optimally influence recovery. We generated the hypotheses that canes which reduce hemiplegic muscle activity, may reduce ipsilesional cortical plasticity and therefore function. TheraTogs which increase hemiplegic muscle activity during walking, may increase ipsilesional cortical plasticity and therefore function. These hypotheses provided the basis for the development of prospective study designs in acute and chronic stroke patients (chapters $7 \& 8$ ).

Chapter 5. In this cross-sectional study in healthy subjects we investigated the immediate effect of walking with a four-wheeled-walker (rollator) on lower-limb muscle activity and trunk-sway, compared to walking with no aids. We found that during rollator use, muscle activity in all muscle groups was reduced in both lower limbs and that increasing the push on the walker through the arms, resulted in further reductions. This increased push replicates the situation in patients who require more support and therefore push more on the rollator. Despite reductions in the hip abductor and extensor muscle activity, which are partly responsible for maintaining a stable pelvis during gait, trunk sway did not increase. It would be expected that pelvic instability caused by re- 
duced activity in the stabilizing musculature, would result in increased trunk sway. As this did not occur, other mechanisms must have been responsible for maintaining stability. We postulated that increased activity at the arms when pushing on the rollator frame, compensated for reduced activity at the lower extremities. Rollator walking may therefore cause reduced muscle activity in the lower extremities and abnormal balance reactions as stability is gained via compensatory activity at the arms. These findings need to be investigated further in patient groups and long term consequences should be studied. Rollator walking may be beneficial in patients who would otherwise not walk at all, but possibly is deleterious in patients who are able to walk without or with other aids.

Chapter 6. In this case report, a patient with delayed healing of a conservatively treated avulsion fracture of the greater trochanter 12 weeks previously and with a 14year history of total hip arthroplasty was presented. We systematically documented the effect of walking with crutches, an orthotic garment TheraTogs, and no walking aids over three 4-week periods on walking speed, trunk sway, and muscle activity measured with EMG. We found that walking speed improved at a faster rate in the TheraTogs phase than in the crutches phase and that walking speed reduced in no-walking-aids phase. Trunk sway increased in the crutch and no-aids phases, and became more stable in the TheraTogs phase. In this patient, function and recovery rate of all measured parameters increased more in the TheraTogs phase than when walking with crutches or during the no-aids phase. We postulated that this may have been because crutch walking unloaded the affected hip and the recovering structures, thereby enabling walking, but without facilitating muscle activity in the affected structures. TheraTogs on the other hand facilitated muscle activity, thereby enabling active support of recovering structures. No walking aids provided no support, so that affected structures became inflamed and walking was painful. This case report provided insight into the prospective, on-going influence of walking aids on muscle activity and function during rehabilitation in a patient with an intact nervous system.

Chapter 7. In this chapter we described the study protocol for a multi-centered randomized control trial in acute stroke patients, in which the effect of early gait rehabilitation with two different walking aids, canes and an orthotic garment TheraTogs, on the recovery of gait function and balance would be compared. This was the first protocol that we were aware of to investigate the long-term influence of walking aids in rehabilitation in post-stroke patients. It was also the first study on walking aids in which the hypotheses were based on an understanding of mechanisms influencing neuroplasticity, motor learning and functional recovery post-stroke.

Chapter 8. In this multiple single case study with four cane using, chronic stroke patients we experimentally investigated the long-term effect of reducing cane use as much as possible and replacing it with the orthosis TheraTogs, on gait function and balance. During a baseline period subjects walked with canes as usual. Gait function and trunk sway were measured weekly. During intervention periods of varying and 
randomized time spans, cane use was reduced as much as possible and the orthotic garment TheraTogs was worn daily from morning to evening. Subjects continued to be measured weekly. In a follow-up period subjects either returned to cane use, continued to use TheraTogs or walked with no aids, depending on their functional abilities and personal preferences. All patients improved in gait function, two showed improvements larger than a Clinically Important Minimal Difference, one of whom became an independent walker without walking aids. Trunk sway also improved but changes were not statistically significant. These results indicated that walking-aids decisively impact gait and balance ability. Walking-aids which increase muscle activity, allow normal jointloading and do not require hands may be more effective than canes for gait rehabilitation. Further research is needed to confirm these results, to identify which patients benefit and to optimize further aspects of intervention (e.g. duration).

Chapter 9. In this chapter a general discussion of the overall findings was presented. Implications for clinical practice, the formulation of clinical practice guidelines and future research were examined. Insights gained throughout the research process, which were not defined as aims at the outset, such as the influence of study design on results and the optimal designs for rehabilitation research, were also discussed. 

Valorization 
The long-term impact of walking aids on muscle activity, gait and balance has been sparsely investigated. This thesis used diverse study designs in different patient groups including an experimental, prospective study design in stroke patients, to generate new knowledge regarding the impact of various walking aids on muscle activity, gait and balance recovery. In addition to the clinical aspects of these investigations already discussed in earlier chapters, this valorization will consider the economic and societal relevance of the findings in this thesis as well as innovations made.

\section{INNOVATION}

Walking aids including canes and rollators are prescribed during the rehabilitation of many patient groups although clear evidence regarding the long-term impact of walking aid use is often lacking. In this thesis, cross-sectional and prospective designs were used to investigate these effects with an emphasis on post-stroke gait and balance recovery.

Epidemiological studies indicate that two thirds of people with a stroke will use a cane. [1] This intervention is supported by Clinical Practice Guidelines which recommend the use of canes following cardiovascular insult to improve balance, stability and function. [2] Despite this, physiotherapists are often reluctant to provide canes or rollators to patients, particularly during the acute and sub-acute phases, believing that usage impedes the recovery of balance and independent walking. This was evident during the recruitment phase of the Randomized Control Trial (RCT) in this thesis (see protocol Chapter 7). Many therapists advised their patients against trial participation due to the possibility of being randomized to a cane group.

These facts indicate a discrepancy between evidence based recommendations and actual clinical practice. It appears that many therapists consider current evidence to be lacking and prefer to work on the basis of clinical experience. This may reflect the fact that current research consists of cross-sectional and observational study results and lacks experimental prospective data.

Robust research results are often difficult to collect during many forms of rehabilitation including post-stroke, due to the high incidence of co-morbidities, the wide spectrum of presenting signs and symptoms and the consequent necessity of a variety of treatments and interventions. Using traditional research methods, particularly RCTs, these points lead either to high contamination and poor internal validity, or in an attempt to address these issues, to a narrow non-representative subject sample. Our replicated single case study attempted to address these challenges by incorporating several innovative steps into the conceptualization and implementation of study methods.

Firstly, hypotheses were generated based not only on clinical experience but also through the consideration of current neuroscientific knowledge. This concrete application of a "bench to bedside" approach, in which facts from academic neuroscience were 
incorporated into the testing of treatments on the clinical frontline, is an aspect of research which has been identified by task-forces as desirable but is often lacking. [3] Secondly the study design - a replicated single case study - was implemented based on our reflections following recruitment difficulties during the initial RCT with acute patients (see protocol Chapter 7) and due to discussions in the literature regarding optimal designs for rehabilitation research. [4, 5]

The results of our study indicate experimentally, for the first time, that cane use following stroke does indeed impede balance and gait recovery, as suspected by many clinical therapists, and in contrast to cross-sectional design results and guideline recommendations. For the first time, to our knowledge, this thesis recommends an alternative to cane use. Although the actual product tested was "TheraTogs", the principles developed in our work that walking aids should not require hand use, should not artificially increase the base of support and should not reduce muscle activity during walking, as canes do, could be used to develop further varieties of walking aid.

Our study also indicates that these factors are relevant not only for acute and subacute patients but also for chronic stroke patients. This is in contrast to studies which indicate that most functional recovery stops between 8 [6] and 20 [7] weeks poststroke.

\section{SOCIETAL AND ECONOMIC RELEVANCE}

The need for cost effective, evidence based treatments in stroke rehabilitation is clear considering the Global Burden of Disease post stroke. A Global Burden of Stroke Study estimated that stroke survivors worldwide number 33 million. Disability Adjusted Life Years (DALYs) lost due to stroke was estimated to be 102 million in 2010, [8] and 80\% of all stroke survivors live in low and middle income economies which struggle to provide rehabilitative care. [9] Economic pressures for health rationing in developed countries also means that effective, non-expensive interventions are gaining in importance.[10]

This thesis has delivered novel, clinically important evidence which may result in improved gait and balance and consequently improved functional independence. Our recommendation that walking aids should not require hand use, has the secondary effect that patients are able to implement the non-hemiplegic hand for functional tasks and not simply to hold aids. Both of these points could help to reduce the burden of health care costs for stroke survivors. The intervention itself, whilst initially requiring more capital outlay that walking aids such as canes or rollators, is cheaper than many newly developed technologies for gait rehabilitation such as robotics or virtual reality. 


\section{TARGET GROUP}

The studies in this thesis indicate that an objective, evidence based prescription and use of walking aids is important in many patient groups including those undergoing accelerated rehabilitation following orthopedic rehabilitation and in stroke survivors.

As we showed that walking aid use can influence gait and balance in chronic stroke patients, it is likely that these effects are also relevant for acute and sub-acute patients. As neuroplastic and neuromuscular changes are not so clearly established in these patients, it is likely that change would be easier to generate. However, this point remains to be established in further studies. It is clear that these results are relevant for large groups of gait impaired individuals.

\section{TRANSFER OF KNOWLEDGE}

Studies have shown that many barriers exist to the implementation of new evidence into clinical practice. [11] Although attitudes to Evidence Based Practice (EBP) are generally positive, the perception exists that research can be distanced from the "real world" and is therefore often difficult to apply.[12-14] Too little time to search and appraise research findings has consistently been identified by practitioners as the main barrier to EBP. [12, 15, 16]

The difficulties of investigating clinically relevant and practically applicable interventions have been partially overcome in this thesis through the use of a real-world study design. Recruited patients were representative of chronic, cane using stroke patients and were not excluded on the basis of strict selection criteria in an attempt to maintain internal validity. It is interesting that the results appear to support the assumptions of many therapists based on clinical experience. The clinical relevance and practicability of these results may assist the willingness of clinicians to implement these new interventions.

As inadequate research and appraisal time and skills of individual studies are often cited by clinicians as a reason for not being aware of recent research, evidence appearing in guidelines or other forms of user-friendly evidence summaries is more likely to be accessed and transferred into clinical practice. It will be important to ensure that the results of our studies, and the results of other observational prospective studies indicating similar findings [17-19] are incorporated into practice guidelines. The current recommendations that cane and rollator use is primarily positive post-stroke, should be updated. Additional forms of evidence summaries, such as those produced by the Joanna Briggs Institute could also be generated to assist the transfer of these important research findings into practice. [20] 


\section{CONCLUSION}

The need for cost-effective and practicable interventions to improve functional independence following stroke continues to grow as the population ages and stroke incidence increases in low and middle income countries. The results of the studies in this thesis help to provide these requirements. We have shown that an inexpensive small change to a treatment regime, which does not require large amounts of therapist input, can significantly improve aspects of gait and balance as well as freeing hands for functional tasks in large numbers of patients. This improvement may increase functional independence thus reducing care costs.

The dissemination of this knowledge through the incorporation into guidelines and evidence summaries will be an important step to facilitate knowledge transfer into clinical practice.

Further studies to identify which patient sub-groups may benefit from these interventions as well as to evaluate the influence on acute and sub-acute stroke patients are necessary.

The principles identified in this thesis for optimal walking aid support could be applied to further develop and innovate assistive walking devices. 


\section{REFERENCES}

1. West BA, Bhat G, Stevens J, Bergen G: Assistive device use and mobility-related factors among adults aged $>$ /=65years. J Safety Res 2015, 55:147-150.

2. Veerbeek J, Wegen Ev, Peppen Rv, Hendriks H, Rietberg M, Wees Pvd, Heijblom K, Goos A, Hanssen W, We BH-vd et al: Royal Dutch Society for Physical Therapy - Clinical Practice Guideline for Physical Therapy in patients with stroke. 2014.

3. Cheeran B, Cohen L, Dobkin B, Ford G, Greenwood R, Howard D, Husain M, Macleod M, Nudo R, Rothwell $\mathrm{J}$ et al: The future of restorative neurosciences in stroke: driving the translational research pipeline from basic science to rehabilitation of people after stroke. Neurorehabil Neural Repair 2009, 23(2):97107.

4. Barnett SD, Heinemann AW, Libin A, Houts AC, Gassaway J, Sen-Gupta S, Resch A, Brossart DF: Small N designs for rehabilitation research. J Rehabil Res Dev 2012, 49(1):175-186.

5. Backman $\mathrm{CL}$, Harris SR: Case studies, single-subject research, and $\mathrm{N}$ of 1 randomized trials: comparisons and contrasts. Am J Phys Med Rehabil 1999, 78(2):170-176.

6. van Kordelaar J, van Wegen E, Kwakkel G: Impact of time on quality of motor control of the paretic upper limb after stroke. Arch Phys Med Rehabil 2014, 95(2):338-344.

7. Jorgensen HS, Nakayama H, Raaschou HO, Vive-Larsen J, Stoier M, Olsen TS: Outcome and time course of recovery in stroke. Part II: Time course of recovery. The Copenhagen Stroke Study. Arch Phys Med Rehabil 1995, 76(5):406-412.

8. Feigin VL, Forouzanfar MH, Krishnamurthi R, Mensah GA, Connor M, Bennett DA, Moran AE, Sacco RL, Anderson L, Truelsen T et al: Global and regional burden of stroke during 1990-2010: findings from the Global Burden of Disease Study 2010. Lancet 2014, 383(9913):245-254.

9. Feigin VL, Mensah GA, Norrving B, Murray CJ, Roth GA: Atlas of the Global Burden of Stroke (1990-2013): The GBD 2013 Study. Neuroepidemiology 2015, 45(3):230-236.

10. Rooshenas L, Owen-Smith A, Hollingworth W, Badrinath P, Beynon C, Donovan JL: "I won't call it rationing...": an ethnographic study of healthcare disinvestment in theory and practice. Soc Sci Med 2015, 128:273-281.

11. Scurlock-Evans L, Upton P, Upton D: Evidence-Based Practice in physiotherapy: a systematic review of barriers, enablers and interventions. Physiotherapy 2014, 100(3):208-219.

12. Heiwe S, Kajermo KN, Tyni-Lenne R, Guidetti S, Samuelsson M, Andersson IL, Wengstrom Y: Evidencebased practice: attitudes, knowledge and behaviour among allied health care professionals. Int J Qual Health Care 2011, 23(2):198-209.

13. Boonstra TW, Daffertshofer A, Roerdink M, Flipse I, Groenewoud K, Beek PJ: Bilateral motor unit synchronization of leg muscles during a simple dynamic balance task. In: Eur J Neurosci. Volume 29, edn. France; 2009: 613-622.

14. Karin H, Filip S, Jo G, Bert A: Obstacles to the implementation of evidence-based physiotherapy in practice: a focus group-based study in Belgium (Flanders). Physiother Theory Pract 2009, 25(7):476-488.

15. Jette DU, Bacon K, Batty C, Carlson M, Ferland A, Hemingway RD, Hill JC, Ogilvie L, Volk D: Evidencebased practice: beliefs, attitudes, knowledge, and behaviors of physical therapists. Phys Ther 2003, 83(9):786-805.

16. Scurlock-Evans L, Upton P, Upton D: Evidence-Based Practice in physiotherapy: a systematic review of barriers, enablers and interventions. Physiotherapy 2014

17. Hamzat TK, Kobiri A: Effects of walking with a cane on balance and social participation among communitydwelling post-stroke individuals. Eur J Phys Rehabil Med 2008, 44(2):121-126.

18. Kim O, Kim JH: Falls and Use of Assistive Devices in Stroke Patients with Hemiparesis: Association with Balance Ability and Fall Efficacy. Rehabil Nurs 2015, 40(4):267-274.

19. Son SM, Kwon JW, Nam SH, Lee NK, Kim K, Kim CS: Adverse effects of motor-related symptoms on the ipsilateral upper limb according to long-term cane usage. NeuroRehabilitation 2012, 31(2):137-141. 
20. Pearson A, Jordan Z, Munn Z: Translational science and evidence-based healthcare: a clarification and reconceptualization of how knowledge is generated and used in healthcare. Nurs Res Pract 2012, 2012:792519. 



\section{Acknowledgements}

I was told before I embarked on my PhD odyssey to be prepared to give up weekends, holidays and peace of mind for the duration. Like many optimistic or naïve beginners, I dismissed the comments as exaggerated and certainly not applicable to me. As it turns out they were largely true, which means that for the last six years I have often been a physically or mentally absent member of my family. Despite this, they never questioned my decision to begin a PhD, rather just pulled together and got on with it (perhaps they were glad to get rid of me every now and again). Without their steadfast trust and support I would never have succeeded. So thank you Hannah and Ciara for always doing your own washing, thank you Rory for learning to fry all forms of human foodstuff and thank you Denis for working so hard that I had the space and time I needed.

Amongst the countless things I have learned on this journey, many of the most important are due to the extremely competent and patient supervision of Judith Sieben. Thank you for always replying knowledgeably and promptly (never to be underestimated) to my questions and with feedback. Massive thanks for helping me on the occasions when I really reached a cul-de-sac, for having an open ear and for the invaluable, solution-seeking discussions. Thanks for finding additional expertise when we needed assistance and huge amounts of thanks as well for your personal support, such as during my crowdfunding project which enabled me to buy essential measurement equipment.

I also owe a debt of gratitude to Rob de Bie for his humorous and intelligent support and feedback. With a couple of precise and incisive sentences, your feedback often exposed the flaws or potential improvements in a line of reasoning. Thank you for agreeing to support the project in the initial stages and for sticking with it despite the various twists and turns, ups and downs of the research process.

Trust and belief have been an important part of the whole PhD process. This is especially true of the people who supported my very first study protocol, enabling me to apply for a PhD position and allowing my job and work to be structured in such a way as to make this project possible. I would therefore like to give a huge thanks to Heike Scheidhauer for her trust and support and to Hans-Peter Karrer for his confidence and backing. Without the assistance and encouragement provided by the Bildungszentrum Gesundheit Basel-Stadt, due to the endorsement of Heike and Hans-Peter, the project could not have been initiated or maintained. Likewise, great thanks to the physiotherapy team who have assisted and sustained me in many different ways throughout the 
whole process. Special thanks to Martina for reading manuscripts and giving feedback, to Anke and Nathanael for helping with measurements, to Dorothea for so much commitment during the crowdfunding project and to Lukas for frequent advice and suggestions.

Without the co-operation of the staff in the hospitals and clinics who helped with the discussions regarding practicability of study design, with recruitment and with interventions, none of the clinical research would have been possible. A particular thank you to Florian Erzer for enabling and supporting research at the Rehab in Basel and to Gisela van der Wijden for her invaluable work. Thank you to the staff at all the clinics involved in the RCT particularly Charlotte Wälti, Ida Dommen and Bernadette Vögele from the Kantonspital Luzern, Götz Hasenberg at the Reha Klinik in Bad Zurzach. Annelies Tobler, Claire Liniger and Fabienne Sauser from the Kantospital Bruderholz as well as Matthias Frank and Hans-Ueli Schläpffer from Felix-Platter Spital in Basel.

It's clear that research is not the undertaking of an individual. I have mentioned some of the people and organisations instrumental in enabling me to carry out the research in this thesis. There are however many more. I am very grateful to the following organisations: to the "Physiotherapie Wissenschaften (PTW) Stiftung" which awarded me a research prize enabling me to buy electromyographical and other essential equipment to measure outcomes, to "physioswiss" who also supported me financially, to the physiotherapy department of Claraspital who supported the crowdfunding appeal and to Barbara Würth who organized the event, to the physiotherapy department of Bruderholzspital who also supported the crowdfunding appeal. I would also like to say a big thank you to Rosa and Georg Ferber for providing statistical support and for generously contributing to the crowdfunding project. And to everybody else who supported the crowdfunding appeal including Jürg and Christine.

Thanks to my brother and sisters. Knowing that you are all there, only a phone call (or a What's app) away, is always a massive help.

And last but most definitely not least I would like to thank my mum. Thanks for the love, stability, understanding and acceptance that was always there.

And of course hello to dad who would be very proud. 


\section{About the Author Curriculum Vitae}

Clare Maguire was born in Manchester UK in 1965. She completed her physiotherapy training at Kings College Hospital in London in 1989. After qualification she worked at various hospitals in London, Manchester and Boston USA. In 1999 she moved to Switzerland where she also worked as a physiotherapist before becoming a physiotherapy lecturer in 2008.

In 1999 she completed a programme in Neurological Dysfunction at the University of Salford, UK. In 2008 she received a Master of Physiotherapy Science degree awarded jointly by the Maastricht and Zürich universities. In 2011 she completed the Lecturer Teaching Programme at the University of Basel and in 2012 completed a "How to teach Evidence Based Medicine" programme at Oxford University UK.

Clare is currently a lecturer in the physiotherapy Bachelor Degree programme of the Bern University of Applied Science based at the Bildungszentrum Gesundheit BaselStadt (BZG). She is Head of the Neurological Rehabilitation Master Programme of the Bern and Zürich Universities of Applied Science and is Head of Physiotherapy Research at BZG. 



\section{Publications}

- Maguire C, Sieben JM, Scheidhauer H, Romkes J, Suica Z, de Bie RA. The effect of crutches, an orthosis TheraTogs, and no walking aids on the recovery of gait in a patient with delayed healing post hip fracture: A case report. Physiother Theory Pract. 2016 Jan;32(1):69-81.

- Suica Z, Romkes J, Tal A, Maguire C. Walking with a four wheeled walker (rollator) significantly reduces EMG lower-limb muscle activity in healthy subjects. Journal of Bodywork \& Movement Therapies. 2016 20, 65e73Abstract

- Maguire C, Sieben JM, Erzer F, Goepfert B, Frank M, Ferber G, Jehn M, SchmidtTrucksäss A, de Bie RA. How to improve walking, balance and social participation following stroke: a comparison of the long term effects of two walking aids - canes and an orthosis TheraTogs - on the recovery of gait following acute stroke. A study protocol for a multi-centre, single blind, andomised control trial. BMC Neurol. 2012 Mar $30 ; 12: 18$

- Maguire C, Sieben JM, Frank M, Romkes J. Hip abductor control in walking following stroke -- the immediate effect of canes, taping and TheraTogs on gait. Clin Rehabil. 2010 Jan;24(1):37-45.

\section{CONFERENCE CONTRIBUTIONS}

- Physioswiss Kongress 2016 - 2 presentations - Stroke and Clinical Reasoning

- World Congress for Neurorehabilitation, Philadelphia USA 2016 - poster - Gait, Stroke

- World Conress for Stroke Rehabilitation, Istanbul 2014 - poster -Gait, Stroke

- Physioswiss Kongress 2014 - poster \& workshop -Single Case Studies

- 17. Deutsch Mukovizidose Kongress - invited presentation -Clinical Reasoning

- 16. Basler Symposium für Kinderorthopädie 2013 -invited presentation -Walking Aids

- Physioswiss Kongress 2010 - presentation - Gait, Stroke

- Reha Schweiz Kongress 2009 - poster - Gait, Stroke 